\title{
Formal Total Syntheses of Aspidosperma Alkaloidsvia a Novel and General Synthetic Pathway Based on an Intramolecular Heck Cyclization
}

\author{
J. Pereira, M. Barlier and C. Guillou * \\ Institut de Chimie des Substances Naturelles, CNRS Avenue de la Terrasse 91198 Gif-sur-Yvette, \\ France. \\ guillou@icsn.cnrs-if.fr
}

Supporting information 


\section{Table of Contents}

General Procedure for the Synthesis of Acetamide 10a-c S4

$\begin{array}{ll}\text { Preparation of compound 10a } & \text { S4 }\end{array}$

$\begin{array}{ll}\text { Preparation of compound 10b } & \text { S4 }\end{array}$

$\begin{array}{ll}\text { Preparation of compound 10c } & \text { S5 }\end{array}$

$\begin{array}{ll}\text { Preparation of compound 10d } & \text { S6 }\end{array}$

$\begin{array}{ll}\text { Preparation of compound 10e } & \text { S7 }\end{array}$

General Procedure for the Synthesis of Quinolinone 13a-e S8

$\begin{array}{ll}\text { Preparation of compound 13a } & \text { S8 }\end{array}$

$\begin{array}{ll}\text { Preparation of compound 13b } & \text { S9 }\end{array}$

$\begin{array}{ll}\text { Preparation of compound 13c } & \text { S10 }\end{array}$

$\begin{array}{ll}\text { Preparation of compound 13d } & \text { S11 }\end{array}$

$\begin{array}{ll}\text { Preparation of compound 13e } & \text { S12 }\end{array}$

General Procedure for the Synthesis of carbamate derivatives 14a-c S13

$\begin{array}{ll}\text { Preparation of compound 14a } & \text { S13 }\end{array}$

$\begin{array}{ll}\text { Preparation of compound 14b } & \text { S14 }\end{array}$

$\begin{array}{ll}\text { Preparation of compound 14c } & \text { S15 }\end{array}$

$\begin{array}{ll}\text { General Procedure for the Synthesis of quinolinones 15a-c } & \text { S16 }\end{array}$

$\begin{array}{ll}\text { Preparation of compound 15a } & \text { S16 }\end{array}$

$\begin{array}{ll}\text { Preparation of compound 15b } & \text { S17 }\end{array}$

$\begin{array}{lr}\text { Preparation of compound 15c } & \text { S17 }\end{array}$

$\begin{array}{ll}\text { General Procedure for the Synthesis of carbamates derivatives 9a-c } & \text { S18 }\end{array}$

$\begin{array}{ll}\text { Preparation of compound 9a } & \text { S18 }\end{array}$

$\begin{array}{ll}\text { Preparation of compound } 9 \mathbf{b} & \text { S19 }\end{array}$

$\begin{array}{lr}\text { Preparation of compound 9c } & \text { S20 }\end{array}$ 


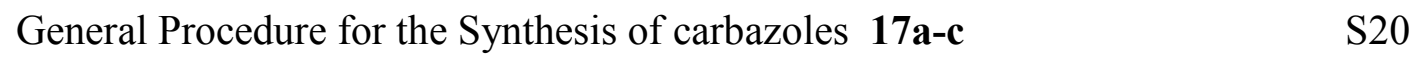

$\begin{array}{ll}\text { Preparation of compound 17a } & \text { S21 }\end{array}$

$\begin{array}{ll}\text { Preparation of compound } \mathbf{1 7 b} & \text { S21 }\end{array}$

$\begin{array}{ll}\text { Preparation of compound 17c } & \text { S22 }\end{array}$

General Procedure for the Synthesis of carbazoles 18a-c and 19a-c S23

$\begin{array}{ll}\text { Preparation of compound 18a } & \text { S23 }\end{array}$

$\begin{array}{ll}\text { Preparation of compound 18b } & \text { S24 }\end{array}$

$\begin{array}{ll}\text { Preparation of compound 18c } & \text { S25 }\end{array}$

$\begin{array}{ll}\text { Preparation of compound 19a } & \text { S26 }\end{array}$

$\begin{array}{ll}\text { Preparation of compound 19b } & \text { S27 }\end{array}$

$\begin{array}{ll}\text { Preparation of compound 19c } & \text { S28 }\end{array}$

$\begin{array}{ll}\text { General Procedure for the Synthesis of carbazoles 20a-c } & \text { S29 }\end{array}$

$\begin{array}{ll}\text { Preparation of compound 20a } & \text { S29 }\end{array}$

$\begin{array}{ll}\text { Preparation of compound 20b } & \text { S30 }\end{array}$

$\begin{array}{ll}\text { Preparation of compound 20c } & \text { S31 }\end{array}$

$\begin{array}{ll}\text { General Procedure for the Synthesis of carbazoles 21a-c } & \text { S32 }\end{array}$

$\begin{array}{ll}\text { Preparation of compound 21a } & \text { S33 }\end{array}$

$\begin{array}{ll}\text { Preparation of compound 21b } & \text { S33 }\end{array}$

$\begin{array}{lr}\text { Preparation of compound 21c } & \text { S34 }\end{array}$

$\begin{array}{ll}\text { General Procedure for the Synthesis of carbazoles 22a-c } & \text { S35 }\end{array}$

$\begin{array}{ll}\text { Preparation of compound 22a } & \text { S35 }\end{array}$

$\begin{array}{ll}\text { Preparation of compound 22b } & \text { S36 }\end{array}$

$\begin{array}{ll}\text { Preparation of compound 22c } & \text { S36 }\end{array}$

$\begin{array}{ll}\text { General Procedure for the Synthesis of carbazoles 23a-c } & \text { S37 }\end{array}$

$\begin{array}{ll}\text { Preparation of compound 23a } & \text { S38 }\end{array}$

$\begin{array}{ll}\text { Preparation of compound 23b } & \text { S38 }\end{array}$

$\begin{array}{lr}\text { Preparation of compound 23c } & \text { S39 }\end{array}$

$\begin{array}{ll}\text { General Procedure for the Synthesis of carbazoles 8a-c } & \text { S40 }\end{array}$

$\begin{array}{ll}\text { Preparation of compound 8a } & \text { S40 }\end{array}$

$\begin{array}{ll}\text { Preparation of compound } \mathbf{8 b} & \text { S41 }\end{array}$

$\begin{array}{ll}\text { Preparation of compound 8c } & \text { S41 }\end{array}$

$\begin{array}{ll}\text { Spectral copies } & \text { S42-S85 }\end{array}$ 


\section{General Procedure for the Synthesis of Acetamide 10a-c}

To a mixture of (1,4-dioxa-spiro[4.5]dec-7-en-8-yl)-acetic acid 11, the corresponding iodo-aniline and 2-chloro-1-methylpyridinium iodide dissolved in dry $\mathrm{CH}_{2} \mathrm{Cl}_{2}$, was added $\mathrm{NEt}_{3}$. The reaction mixture was heated for 15 hours. The reaction mixture was extracted with $\mathrm{CH}_{2} \mathrm{Cl}_{2}$. The combined organic layer was washed with $\mathrm{H}_{2} \mathrm{O}$, brine and dried $\left(\mathrm{MgSO}_{4}\right)$ and evaporated. Silica gel flash-column chromatography of the residue afforded 10a-c as indicated below for each case.

2-(1',4'-dioxa-spiro[4.5]dec-7'-en-8'-yl)- $N$-(2-iodo-phenyl)-acetamide 10a

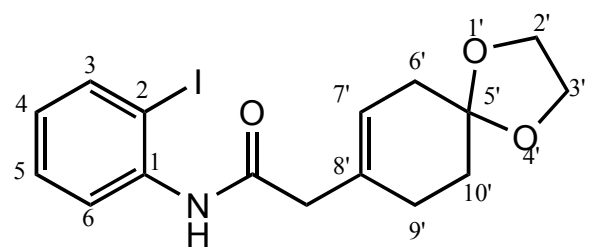

$10 \mathbf{a}$

(1,4-dioxa-spiro[4.5]dec-7-en-8-yl)-acetic acid 11 (1 g, 5.05 mmol), 2-iodophenylamine 12a (1,55 g, $7.07 \mathrm{mmol})$, 2-chloro-1-methylpyridinium iodide (1.94 g, $7.58 \mathrm{mmol})$, dry $\mathrm{CH}_{2} \mathrm{Cl}_{2}(88 \mathrm{~mL})$ and $\mathrm{NEt}_{3}(2.46 \mathrm{~mL}, 17.70 \mathrm{mmol})$ were used to produce 10a. Purification : Heptane/EtOAc (7:3). White powder (1.83 g, 91\%).

H.R.M.S. (E.S.I., $\mathrm{m} / \mathrm{z}$ ) calcd for $\mathrm{C}_{16} \mathrm{H}_{18} \mathrm{INNaO}_{3}{ }^{+}\left(\mathrm{MNa}^{+}\right): 422.0229$, found : 422.0243 .

I.R. $\left(\mathrm{CHCl}_{3}\right) \vee\left(\mathrm{cm}^{-1}\right): 3322(\mathrm{~N}-\mathrm{H}), 1681(\mathrm{C}=\mathrm{O}$ (amide)), $1511(\mathrm{C}=\mathrm{C}), 1427(\mathrm{C}-\mathrm{Car})$, 1109 (C-O).

M.S. (E.S.I., $\mathrm{m} / \mathrm{z}): 422\left(\mathrm{MNa}^{+}\right)$.

1H RMN $\left(\mathrm{CDCl}_{3}, 300 \mathrm{MHz}\right) \delta(\mathrm{ppm}): 8.29\left(1 \mathrm{H}, \mathrm{dd}, \mathrm{J}=8.2 \mathrm{~Hz}, \mathrm{~J}=1.2 \mathrm{~Hz}, \mathrm{H}_{6}\right), 8.02$ $(1 \mathrm{H}, \mathrm{sl}, \mathrm{NH}), 7.76\left(1 \mathrm{H}, \mathrm{dd}, \mathrm{J}=7.9 \mathrm{~Hz}, \mathrm{~J}=1.4 \mathrm{~Hz}, \mathrm{H}_{3}\right), 7.34(1 \mathrm{H}, \mathrm{dt}, \mathrm{J}=8.5 \mathrm{~Hz}, \mathrm{~J}=1.4$ $\left.\mathrm{Hz}, \mathrm{H}_{5}\right), 6.83\left(1 \mathrm{H}, \mathrm{dt}, \mathrm{J}=7.8 \mathrm{~Hz}, \mathrm{~J}=1.3 \mathrm{~Hz}, \mathrm{H}_{4}\right), 5.77\left(1 \mathrm{H}, \mathrm{s}, \mathrm{H}_{7}\right), 4.00\left(4 \mathrm{H}, \mathrm{s}, \mathrm{H}_{2}\right.$, and $\mathrm{H}_{3}$ ) $), 3.16\left(2 \mathrm{H}, \mathrm{s}, \mathrm{CH}_{2}\right), 2.42\left(2 \mathrm{H}, \mathrm{s}, \mathrm{H}_{6^{\prime}}\right), 2.34\left(2 \mathrm{H}, \mathrm{m}, \mathrm{H}_{9}{ }^{\prime}\right), 1.88(2 \mathrm{H}, \mathrm{t}, \mathrm{J}=6.4 \mathrm{~Hz}$, $\left.\mathrm{H}_{10}\right)$.

${ }^{13} \mathbf{C}$ RMN $\left(\mathrm{CDCl}_{3}, 75 \mathrm{MHz}\right) \delta(\mathrm{ppm}): 169.1$ (C (O) NH), $138.9\left(\mathrm{C}_{6}\right), 138.3\left(\mathrm{C}_{1}\right), 132.3$ $\left(\mathrm{C}_{8}\right), 129.3\left(\mathrm{C}_{4}\right), 126.4\left(\mathrm{C}_{7}\right), 125.9\left(\mathrm{C}_{5}\right), 121.6\left(\mathrm{C}_{3}\right), 107.5\left(\mathrm{C}_{5}\right), 89.2\left(\mathrm{C}_{2}\right), 64.5\left(\mathrm{C}_{2}\right)$ and $\left.\mathrm{C}_{3}\right), 46.8\left(\mathrm{CH}_{2}\right), 36.0\left(\mathrm{C}_{6}\right)$. 


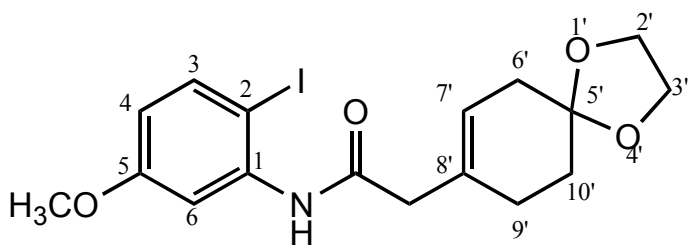

10b

(1,4-dioxa-spiro[4.5]dec-7-en-8-yl)-acetic acid 11 (5 g, $25.25 \mathrm{mmol}$ ), 2-iodo-5methoxy-phenylamine 12b (8.8 g, $35.35 \mathrm{mmol})$, 2-chloro-1-methylpyridinium iodide (9.68 g, $37.89 \mathrm{mmol})$, dry $\mathrm{CH}_{2} \mathrm{Cl}_{2}(250 \mathrm{~mL})$, and $\mathrm{NEt}_{3}(12.3 \mathrm{~mL}, 88.38 \mathrm{mmol})$ were used to produce 10b. Purification : Heptane/EtOAc (7:3). White powder (8.55 g, 79\%). H.R.M.S. (E.S.I., m/z) calcd for $\mathrm{C}_{17} \mathrm{H}_{20} \mathrm{INNaO}_{4}{ }^{+}\left(\mathrm{MNa}^{+}\right) 452.0335$, found : 452.0341 . I.R. $\left(\mathrm{CHCl}_{3}\right) \vee\left(\mathrm{cm}^{-1}\right): 3270(\mathrm{~N}-\mathrm{H}), 1657(\mathrm{C}=\mathrm{O}$ (amide)), $1529(\mathrm{C}=\mathrm{C}), 1469$ (C-Car), 1309 (Car-O), 1109 (C-O).

M.S. (E.S.I., $\mathrm{m} / \mathrm{z}): 452\left(\mathrm{MNa}^{+}\right)$.

${ }_{1} \mathbf{H}$ RMN $\left(\mathrm{CDCl}_{3}, 300 \mathrm{MHz}\right) \delta(\mathrm{ppm}): 8.08\left(1 \mathrm{H}, \mathrm{d}, \mathrm{J}=2.9 \mathrm{~Hz}, \mathrm{H}_{6}\right), 8.03(1 \mathrm{H}, \mathrm{sl}, \mathrm{NH})$, $7.60\left(1 \mathrm{H}, \mathrm{d}, \mathrm{J}=8.8 \mathrm{~Hz}, \mathrm{H}_{3}\right), 6.47\left(1 \mathrm{H}, \mathrm{dd}, \mathrm{J}=8.8 \mathrm{~Hz}, \mathrm{~J}=3.0 \mathrm{~Hz}, \mathrm{H}_{4}\right), 5.77\left(1 \mathrm{H}, \mathrm{s}, \mathrm{H}_{7}{ }^{\prime}\right)$, $4.01\left(4 \mathrm{H}, \mathrm{s}, \mathrm{H}_{2}\right.$, and $\left.\mathrm{H}_{3},\right), 3.81\left(3 \mathrm{H}, \mathrm{s}, \mathrm{OCH}_{3}\right), 3.16\left(2 \mathrm{H}, \mathrm{s}, \mathrm{CH}_{2}\right), 2.43\left(2 \mathrm{H}, \mathrm{s}, \mathrm{H}_{6},\right), 2.34$ $\left(2 \mathrm{H}, \mathrm{m}, \mathrm{H}_{9}\right), 1.89\left(2 \mathrm{H}, \mathrm{t}, \mathrm{J}=6.5 \mathrm{~Hz}, \mathrm{H}_{10}\right)$.

${ }^{13} \mathbf{C}$ RMN $\left(\mathrm{CDCl}_{3}, 75 \mathrm{MHz}\right) \delta(\mathrm{ppm}): 169.2(\mathrm{C}(\mathrm{O}) \mathrm{NH}), 160.7\left(\mathrm{C}_{5},\right), 139.1\left(\mathrm{C}_{1}\right), 138.8$ $\left(\mathrm{C}_{3}\right), 132.3\left(\mathrm{C}_{8}\right), 126.6\left(\mathrm{C}_{7}\right), 112.9\left(\mathrm{C}_{4}\right), 107.5\left(\mathrm{C}_{5}\right), 106.7\left(\mathrm{C}_{6}\right), 64.6\left(\mathrm{C}_{2}\right.$, and $\left.\mathrm{C}_{3^{\prime}}\right)$, $55.6\left(\mathrm{OCH}_{3}\right), 46.9\left(\mathrm{CH}_{2}\right), 36.0\left(\mathrm{C}_{6}\right), 31.2\left(\mathrm{C}_{10}\right), 28.1\left(\mathrm{C}_{9}\right)$.

2-(1',4'-dioxa-spiro[4.5]dec-7'-en-8'-yl)- $N$-(2-iodo-6-methoxy-phenyl)-acetamide 10c<smiles>COc1cccc(I)c1NC(=O)CC1=CCC2([CH-]CO2)CC1</smiles>

(1,4-dioxa-spiro[4.5]dec-7-en-8-yl)-acetic acid 11 (1.7 g, 8.60 mmol), 2-iodo-6methoxy-phenylamine 12c ( $3 \mathrm{~g}, 12.00 \mathrm{mmol}$ ), 2-chloro-1-methylpyridinium iodide (3.3 g, $12.90 \mathrm{mmol})$, dry $\mathrm{CH}_{2} \mathrm{Cl}_{2}(150 \mathrm{~mL})$, and $\mathrm{NEt}_{3}(4.2 \mathrm{~mL}, 30.10 \mathrm{mmol})$ were used to 
produce 10c. Purification : Heptane/EtOAc (4:6). Pale yellow amorphous solid (2.95 g, $80 \%)$.

Anal. C 47,39\%, H 4,59\%, N 3,01\%, O 15,16\% calcd for $\mathrm{C}_{17} \mathrm{H}_{20} \mathrm{INO}_{4} \mathrm{C} 47,57 \%, \mathrm{H}$ 4,70\%, N 3,26\%, O 14,91\%.

I.R. $\left(\mathrm{CHCl}_{3}\right) \vee\left(\mathrm{cm}^{-1}\right)$ : $3382(\mathrm{~N}-\mathrm{H}), 1687(\mathrm{C}=\mathrm{O}$ (amide)), $1554(\mathrm{C}=\mathrm{C}), 1465(\mathrm{C}-\mathrm{Car})$, 1261 (Car-O), 1092 (C-O).

M. S. (E.S.I., m/z): $430\left(\mathrm{MH}^{+}\right)$.

${ }^{1} \mathbf{H}$ RMN $\left(\mathrm{CDCl}_{3}, 300 \mathrm{MHz}\right) \delta(\mathrm{ppm}): 7.44\left(1 \mathrm{H}, \mathrm{d}, \mathrm{J}=7.7 \mathrm{~Hz}, \mathrm{H}_{3}\right), 7.15(1 \mathrm{H}, \mathrm{sl}, \mathrm{NH})$, $6.97\left(1 \mathrm{H}, \mathrm{t}, \mathrm{J}=8.2 \mathrm{~Hz}, \mathrm{~J}=7.8 \mathrm{~Hz}, \mathrm{H}_{4}\right), 6.89\left(1 \mathrm{H}, \mathrm{d}, \mathrm{J}=7.9 \mathrm{~Hz}, \mathrm{H}_{5}\right), 5.72\left(1 \mathrm{H}, \mathrm{sl}_{1} \mathrm{H}_{7^{\prime}}\right)$, $3.99\left(4 \mathrm{H}, \mathrm{s}, \mathrm{H}_{2}\right.$, and $\left.\mathrm{H}_{3}{ }^{\prime}\right), 3.80\left(3 \mathrm{H}, \mathrm{s}, \mathrm{OCH}_{3}\right), 3.14\left(2 \mathrm{H}, \mathrm{s}, \mathrm{CH}_{2}\right), 2.45\left(2 \mathrm{H}, \mathrm{sl}, \mathrm{H}_{9^{\prime}}\right), 2.37$ $\left(2 \mathrm{H}, \mathrm{sl}, \mathrm{H}_{6^{\prime}}\right), 1.85\left(2 \mathrm{H}, \mathrm{t}, \mathrm{J}=6.4, \mathrm{H}_{10^{\prime}}\right)$.

13. RMN $\left(\mathrm{CDCl}_{3}, 75 \mathrm{MHz}\right) \delta(\mathrm{ppm}): 169.1$ (C (O) NH), $155.4\left(\mathrm{C}_{6}\right), 133.0\left(\mathrm{C}_{1}\right), 130.8$ $\left(\mathrm{C}_{3}\right), 129.6\left(\mathrm{C}_{4}\right), 128.1\left(\mathrm{C}_{8^{\prime}}\right), 125.0\left(\mathrm{C}_{7^{\prime}}\right), 111.7\left(\mathrm{C}_{5}\right), 107.7\left(\mathrm{C}_{5^{\prime}}\right), 99.8\left(\mathrm{C}_{2}\right), 64.5\left(\mathrm{C}_{2}\right.$, and $\left.\mathrm{C}_{3^{\prime}}\right), 56.0\left(\mathrm{OCH}_{3}\right), 45.7\left(\mathrm{CH}_{2}\right), 35.9\left(\mathrm{C}_{6^{\prime}}\right), 31.1\left(\mathrm{C}_{10^{\prime}}\right), 28.0\left(\mathrm{C}_{9^{\prime}}\right)$.

\section{Synthesis of the acetamide 10d}

(1',4'-dioxaspiro[4.5]dec-7'-en-8'-yl)-acetyl(2-iodo-6-methoxy-phenyl)-carboxylic acid tert-butyl ester 10d

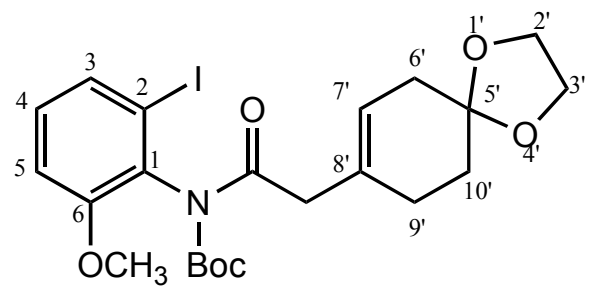

10d

To a solution of 2-(1',4'-dioxa-spiro[4.5]dec-7'-en-8'-yl)- $N$-(2-iodo-6-methoxyphenyl)-acetamide $10 \mathrm{c}(3.01 \mathrm{~g}, 6.99 \mathrm{mmol})$ dissolved in dry $\mathrm{CH}_{2} \mathrm{Cl}_{2}(100 \mathrm{~mL}), \mathrm{NEt}_{3}$ (0.97 mL, $6.99 \mathrm{mmol}), \mathrm{Boc}_{2} \mathrm{O}$ (3.06 g, $\left.14.00 \mathrm{mmol}\right)$, DMAP (856.3 mg, $\left.6.99 \mathrm{mmol}\right)$ were successively added. After being stirred for 16 hours at room temperature, the reaction mixture was extracted with $\mathrm{CH}_{2} \mathrm{Cl}_{2}$. The combined organic layer was washed with $\mathrm{H}_{2} \mathrm{O}$, brine, dried $\left(\mathrm{MgSO}_{4}\right)$ and evaporated. Silica gel flash-column chromatography of the residue (elution with Heptane/EtOAc : 4/6 ) afforded $3.05 \mathrm{~g}$ of (1',4'-dioxaspiro[4.5]dec-7'-en-8'-yl)-acetyl-(2-iodo-6-methoxy-phenyl)-carboxylic acid tert-butyl ester 10d (81\%) as a white oil. 
H.R.M.S. (I.C., $\mathrm{m} / \mathrm{z})$ calcd for $\mathrm{C}_{22} \mathrm{H}_{28} \mathrm{INNaO}_{6}{ }^{+}\left(\mathrm{MNa}^{+}\right)$: 552.0854 , found : 552.0877 .

I.R. $\left(\mathrm{CHCl}_{3}\right) \vee\left(\mathrm{cm}^{-1}\right)$ : $1736(\mathrm{C}=\mathrm{O}(\mathrm{Boc})), 1699(\mathrm{C}=\mathrm{O}$ (amide) $), 1575(\mathrm{C}=\mathrm{C}), 1470(\mathrm{C}$ Car), 1261 (Car-O), 1152 (C-O (Boc)), 1092 (C-O).

M. S. (I.E., m/z) : $529\left(\mathrm{M}^{+}\right.$.).

1H RMN $\left(\mathrm{CDCl}_{3}, 300 \mathrm{MHz}\right) \delta(\mathrm{ppm}): 7.40\left(1 \mathrm{H}, \mathrm{d}, \mathrm{J}=7.9 \mathrm{~Hz}, \mathrm{H}_{3}\right), 7.01(1 \mathrm{H}, \mathrm{t}, \mathrm{J}=7.9$ $\left.\mathrm{Hz}, \mathrm{J}=8.2 \mathrm{~Hz}, \mathrm{H}_{4}\right), 6.89\left(1 \mathrm{H}, \mathrm{d}, \mathrm{J}=8.3 \mathrm{~Hz}, \mathrm{H}_{5}\right), 5.51\left(1 \mathrm{H}, \mathrm{sl}, \mathrm{H}_{7^{\prime}}\right), 3.97\left(4 \mathrm{H}, \mathrm{s}, \mathrm{H}_{2}\right.$, and $\left.\mathrm{H}_{3}\right), 3.77\left(3 \mathrm{H}, \mathrm{s}, \mathrm{OCH}_{3}\right), 3.70\left(2 \mathrm{H}, \mathrm{d}, \mathrm{CH}_{2}\right), 2.30\left(4 \mathrm{H}, \mathrm{sl}, \mathrm{H}_{9^{\prime}}\right.$ and $\left.\mathrm{H}_{6^{\prime}}\right), 1.80(2 \mathrm{H}, \mathrm{t}, \mathrm{J}=$ $\left.6.3 \mathrm{~Hz}, \mathrm{H}_{10^{\prime}}\right), 1.37$ (9H, s, Boc).

${ }^{13} \mathrm{C}$ RMN $\left(\mathrm{CDCl}_{3}, 75 \mathrm{MHz}\right) \delta$ (ppm) : 172.8 (CO (Nboc)), 155.4 (CO (Boc)), 151.4 $\left(\mathrm{C}_{6}\right), 131.7\left(\mathrm{C}_{1}\right), 131.1\left(\mathrm{C}_{3}\right), 130.6\left(\mathrm{C}_{4}\right), 130.3\left(\mathrm{C}_{8^{\prime}}\right), 123.1\left(\mathrm{C}_{7^{\prime}}\right), 111.4\left(\mathrm{C}_{5}\right), 108.0\left(\mathrm{C}_{5^{\prime}}\right)$, $100.7\left(\mathrm{C}_{2}\right), 82.8(\mathrm{C}(\mathrm{Boc})), 64.4\left(\mathrm{C}_{2}\right.$, and $\left.\mathrm{C}_{3}\right), 56.0\left(\mathrm{OCH}_{3}\right), 45.5\left(\mathrm{CH}_{2}\right), 35.9\left(\mathrm{C}_{6^{\prime}}\right), 31.2$ $\left(\mathrm{C}_{10^{\prime}}\right), 27.9\left(\mathrm{CH}_{3}(\mathrm{Boc})\right), 27.9\left(\mathrm{C}_{9^{\prime}}\right)$.

\section{Synthesis of Acetamide 10e}
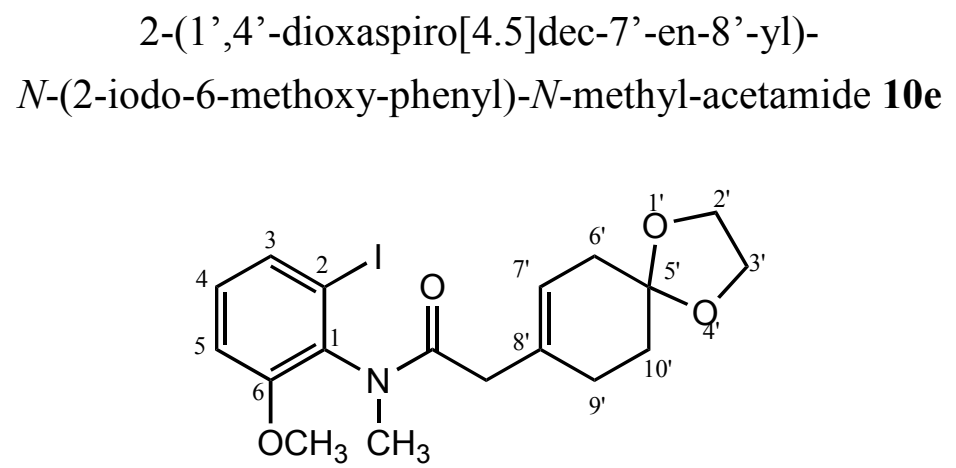

$10 \mathrm{e}$

To a solution of 2-(1',4'-dioxa-spiro[4.5]dec-7'-en-8'-yl)- $N$-(2-iodo-6-methoxyphenyl)-acetamide 10c $(1.88 \mathrm{~g}, 4.39 \mathrm{mmol})$ dissolved in dry THF $(150 \mathrm{~mL})$, a solution of $\mathrm{NaH}(263.0 \mathrm{mg}, 10.98 \mathrm{mmol})$ in dry THF $(120 \mathrm{~mL})$ was added dropwise at $0^{\circ} \mathrm{C}$. After $15 \mathrm{~min}$, the reaction mixture was let go up at room temperature, and $(\mathrm{MeO})_{2} \mathrm{SO}_{2}$ was added $(1.04 \mathrm{~mL}, 0.20 \mathrm{mmol})$. After being stirred for 2 hours at room temperature, $\mathrm{NaHCO}_{3}$ (aq.) was added and the reaction mixture was extracted with $\mathrm{Et}_{2} \mathrm{O}$. The combined organic layer was washed with $\mathrm{H}_{2} \mathrm{O}$, brine, dried $\left(\mathrm{MgSO}_{4}\right)$ and evaporated. Silica gel flash-column chromatography of the residue (elution with Heptane/EtOAc : $4 / 6$ ) afforded $1.67 \mathrm{~g}$ of 2-(1',4'-dioxaspiro[4.5]dec-7'-en-8'-yl)- $N$-(2-iodo-6-methoxyphenyl)- $N$-methyl-acetamide 10e (86\%) as a colourless oil. 
I.R. $\left(\mathrm{CHCl}_{3}\right) \vee\left(\mathrm{cm}^{-1}\right): 1652(\mathrm{C}=\mathrm{O}$ (amide)), $1602(\mathrm{C}=\mathrm{C}), 1470$ (C-Car), 1263 (CarO), 1090 (C-O).

M. S. (E.S.I., m/z): $444\left(\mathrm{MH}^{+}\right)$.

${ }^{1} \mathbf{H}$ RMN $\left(\mathrm{CDCl}_{3}, 300 \mathrm{MHz}\right) \delta(\mathrm{ppm}): 7.45\left(1 \mathrm{H}, \mathrm{d}, \mathrm{J}=8.0 \mathrm{~Hz}, \mathrm{H}_{3}\right), 7.04(1 \mathrm{H}, \mathrm{t}, \mathrm{J}=8.0$ $\left.\mathrm{Hz}, \mathrm{H}_{4}\right), 6.93\left(1 \mathrm{H}, \mathrm{d}, \mathrm{J}=8.0 \mathrm{~Hz}, \mathrm{H}_{5}\right), 5.06\left(1 \mathrm{H}, \mathrm{sl}, \mathrm{H}_{7}\right), 3.94\left(4 \mathrm{H}, \mathrm{s}, \mathrm{H}_{2}\right.$, and $\left.\mathrm{H}_{3}\right), 3.82$ $\left(3 \mathrm{H}, \mathrm{s}, \mathrm{OCH}_{3}\right), 3.09\left(3 \mathrm{H}, \mathrm{s}, \mathrm{NCH}_{3}\right), 2.75-2.63\left(2 \mathrm{H}\right.$, system $\left.\mathrm{AB}, \mathrm{J}=15.0 \mathrm{~Hz}, \mathrm{CH}_{2}\right), 2.21$ $\left(2 \mathrm{H}, \mathrm{sl}, \mathrm{H}_{9^{\prime}}\right), 2.20\left(2 \mathrm{H}, \mathrm{sl}, \mathrm{H}_{6^{\prime}}\right), 1.79\left(2 \mathrm{H}, \mathrm{m}, \mathrm{H}_{10^{\prime}}\right)$.

${ }^{13} \mathbf{C}$ RMN $\left(\mathrm{CDCl}_{3}, 75 \mathrm{MHz}\right) \delta(\mathrm{ppm}): 171.3(\mathrm{C}(\mathrm{O}) \mathrm{NMe}), 156.3\left(\mathrm{C}_{6}\right), 134.8\left(\mathrm{C}_{1}\right)$, $131.8\left(\mathrm{C}_{3}\right), 131.0\left(\mathrm{C}_{4}\right), 130.7\left(\mathrm{C}_{8^{\prime}}\right), 122.5\left(\mathrm{C}_{7}\right), 111.7\left(\mathrm{C}_{5}\right), 107.9\left(\mathrm{C}_{5}\right), 101.7\left(\mathrm{C}_{2}\right), 64.3$ $\left(\mathrm{C}_{2}\right.$, and $\left.\mathrm{C}_{3}{ }^{\prime}\right), 55.9\left(\mathrm{OCH}_{3}\right), 42.3\left(\mathrm{CH}_{2}\right), 35.8\left(\mathrm{C}_{6}\right), 34.5\left(\mathrm{NCH}_{3}\right), 31.0\left(\mathrm{C}_{10}\right), 27.5\left(\mathrm{C}_{9}{ }^{\prime}\right)$.

\section{General Procedure for the Synthesis of Quinolinone 13a-e}

By Heck condition : A mixture of $\mathrm{Pd}_{2}(\mathrm{dba})_{3}$ and dppe in dry DMA was stirred under a oil bath at $110^{\circ} \mathrm{C}$ for $45 \mathrm{~min}$. A solution of 10a-e and PMP was added and the reaction mixture was heated at $110^{\circ} \mathrm{C}$ for 3 hours. After being cooled to room temperature, the reaction mixture was washed by aqueous satured $\mathrm{NaHCO}_{3}$ and was extracted with EtOAc. The combined organic layer was washed with $\mathrm{H}_{2} \mathrm{O}$, brine and dried $\left(\mathrm{MgSO}_{4}\right)$ and evaporated. Silica gel flash-column chromatography of the residue afforded 13a-e as indicated below for each case.

By Heck "ligand free" condition : To a mixture of 10a-c and $\operatorname{Pd}_{2}(\mathrm{dba})_{3}$ in dry DMA, $\mathrm{NEt}_{3}$ was added slowly. The reaction mixture was heated at $140^{\circ} \mathrm{C}$ for two hours. After cooling, the reaction mixture was extracted with EtOAc. The combined organic layer was washed with $\mathrm{H}_{2} \mathrm{O}$, brine, dried $\left(\mathrm{MgSO}_{4}\right)$ and evaporated. Silica gel flash-column chromatography of the residue afforded 13a-c as indicated below for each case.

3-hydro-1H-quinolin-2-one-4,8'-(1',4'-dioxa-spiro[4.5]dec-6'-enyl) 13a 


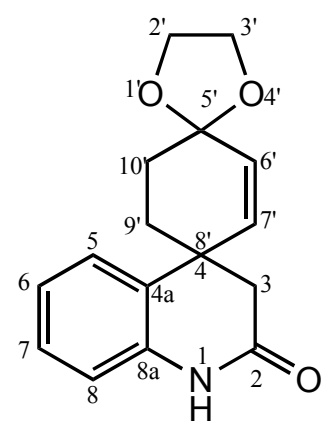

13a

By Heck conditions : $\mathrm{Pd}_{2}(\mathrm{dba})_{3}(5 \mathrm{mg}, 5.5 \mu \mathrm{mol})$, dppe (4.4 mg, $\left.11.0 \mu \mathrm{mol}\right)$, dry DMA (5 mL), 2-(1',4'-dioxa-spiro[4.5]dec-7'-en-8'-yl)- $N$-(2-iodo-phenyl)-acetamide 10a (43.2 $\mathrm{mg}, 0.11 \mathrm{mmol})$, and PMP $(0.08 \mathrm{~mL}, 0.44 \mathrm{mmol})$ were used to produce 13a. Purification : Heptane/EtOAc (4:6). White powder (15.4 mg, 52\%).

By Heck “ligand free" conditions : 2-(1',4'-dioxa-spiro[4.5]dec-7'-en-8'-yl)- $N$-(2iodo-phenyl)-acetamide 10a (3.0 g, $7.52 \mathrm{mmol}), \mathrm{Pd}_{2}(\mathrm{dba})_{3}(68.8 \mathrm{mg}, 0.08 \mathrm{mmol})$, dry DMA $(53 \mathrm{~mL})$, and $\mathrm{NEt}_{3}(3.14 \mathrm{~mL}, 22.60 \mathrm{mmol})$ were used to produce $13 \mathbf{a}$. Purification : Heptane/EtOAc (4:6). White powder (1.86 g, 92\%).

H.R.M.S. (E.S.I., m/z) calcd for $\mathrm{C}_{16} \mathrm{H}_{17} \mathrm{NNaO}_{3}{ }^{+}\left(\mathrm{MNa}^{+}\right)$294.1106, found : 294.1096.

I.R. $\left(\mathrm{CHCl}_{3}\right) \vee\left(\mathrm{cm}^{-1}\right): 3200(\mathrm{~N}-\mathrm{H}), 1668(\mathrm{C}=\mathrm{O}$ (amide)), $1600(\mathrm{C}=\mathrm{C}), 1481(\mathrm{C}-\mathrm{Car})$, 1094 (C-O).

M. S. (E.S.I., m/z) : $294\left(\mathrm{MNa}^{+}\right)$.

1H RMN $\left(\mathrm{CDCl}_{3}, 300 \mathrm{MHz}\right) \delta(\mathrm{ppm}): 8.99(1 \mathrm{H}, \mathrm{sl}, \mathrm{NH}), 7.18\left(2 \mathrm{H}, \mathrm{d}, \mathrm{J}=7.7 \mathrm{~Hz}, \mathrm{H}_{\mathrm{ar}}\right)$, $7.00\left(1 \mathrm{H}, \mathrm{dt}, \mathrm{J}=1.2 \mathrm{~Hz}, \mathrm{~J}=7.6 \mathrm{~Hz}, \mathrm{H}_{\mathrm{ar}}\right), 6.85\left(1 \mathrm{H}, \mathrm{dd}, \mathrm{J}=7.9 \mathrm{~Hz}, \mathrm{~J}=1.5 \mathrm{~Hz}, \mathrm{H}_{\mathrm{ar}}\right)$, $5.91\left(1 \mathrm{H}, \mathrm{d}, \mathrm{J}=10.0 \mathrm{~Hz}, \mathrm{H}_{6}\right), 5.72\left(1 \mathrm{H}, \mathrm{d}, \mathrm{J}=10.0 \mathrm{~Hz}, \mathrm{H}_{7}\right), 4.04-3.90\left(4 \mathrm{H}, \mathrm{m}, \mathrm{H}_{2}\right.$, and $\left.\mathrm{H}_{3}{ }^{\prime}\right), 2.60\left(2 \mathrm{H}\right.$, system $\mathrm{AB}, \mathrm{J}=16.0 \mathrm{~Hz}, \mathrm{H}_{3 \mathrm{a}}$ and $\left.\mathrm{H}_{3 \mathrm{~b}}\right), 2.08-1.82\left(2 \mathrm{H}, \mathrm{m}, \mathrm{H}_{9}{ }^{\prime}\right), 1.80-1.65$ $\left(2 \mathrm{H}, \mathrm{m}, \mathrm{H}_{10}\right)$.

${ }^{13} \mathbf{C ~ R M N}\left(\mathrm{CDCl}_{3}, 75 \mathrm{MHz}\right) \delta(\mathrm{ppm}): 170.2\left(\mathrm{C}_{2}\right), 136.2\left(\mathrm{C}_{4 \mathrm{a}}\right.$ and $\left.\mathrm{C}_{8 \mathrm{a}}\right), 135.8\left(\mathrm{C}_{7}\right)$, $130.7\left(\mathrm{C}_{6}\right), 128.3\left(\mathrm{C}_{\mathrm{ar}}\right), 127.3\left(\mathrm{C}_{\mathrm{ar}}\right), 123.42\left(\mathrm{C}_{\mathrm{ar}}\right), 116.2\left(\mathrm{C}_{\mathrm{ar}}\right), 105.0\left(\mathrm{C}_{5},\right), 64.8$ and 64.7 $\left(\mathrm{C}_{2}\right.$, and $\left.\mathrm{C}_{3^{\prime}}\right), 42.5\left(\mathrm{C}_{3}\right), 39.3\left(\mathrm{C}_{8}\right), 31.6\left(\mathrm{C}_{9}\right), 29.5\left(\mathrm{C}_{10}{ }^{\prime}\right)$.

7-methoxy-3-hydro-1H-quinolin-2-one-4,8'-(1',4'-dioxa-spiro[4.5]dec-6'-enyl) $\mathbf{1 3 b}$ 


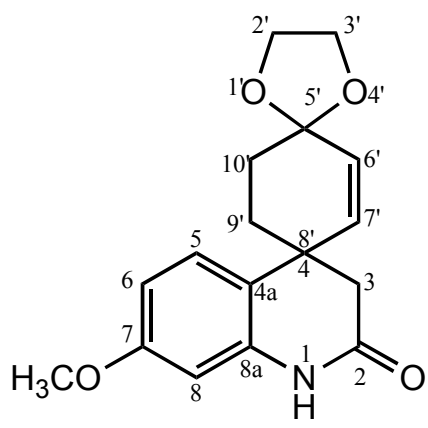

13b

By Heck conditions : $\mathrm{Pd}_{2}(\mathrm{dba})_{3}(5 \mathrm{mg}, 5.5 \mu \mathrm{mol})$, dppe $(4.4 \mathrm{mg}, 11.0 \mu \mathrm{mol})$, dry DMA (5 mL), 2-(1',4'-dioxa-spiro[4.5]dec-7'-en-8'-yl)- $N$-(2-iodo-phenyl)-acetamide 10b (47.2 $\mathrm{mg}, 0.11 \mathrm{mmol})$, and PMP $(0.08 \mathrm{~mL}, 0.44 \mathrm{mmol})$ were used to produce $\mathbf{1 3 \mathbf { b }}$. Purification : Heptane/EtOAc (4:6). White powder (29.1 mg, 88\%).

By Heck “ligand free” conditions : 2-(1',4'-dioxa-spiro[4.5]dec-7'-en-8'-yl)- $N$-(2iodo-5-methoxy-phenyl)-acetamide $10 b$ (4.05 g, $9.44 \mathrm{mmol}), \operatorname{Pd}_{2}(\mathrm{dba})_{3}(86.4 \mathrm{mg}, 0.09$ mmol), dry DMA $(65 \mathrm{~mL})$, and $\mathrm{NEt}_{3}(3.9 \mathrm{~mL}, 28.32 \mathrm{mmol})$ were used to produce $\mathbf{1 3 \mathbf { b }}$. Purification : Heptane/EtOAc (4:6). White powder (2.46 g, 87\%).

H.R.M.S. (E.S.I., m/z) calcd for $\mathrm{C}_{17} \mathrm{H}_{19} \mathrm{NNaO}_{4}{ }^{+}\left(\mathrm{MNa}^{+}\right)$324.1212, found : 324.1218 .

I.R. $\left(\mathrm{CHCl}_{3}\right) \vee\left(\mathrm{cm}^{-1}\right): 2923(\mathrm{~N}-\mathrm{H}), 1681(\mathrm{C}=\mathrm{O}$ lactame $), 1591(\mathrm{C}=\mathrm{C}), 1265(\mathrm{Car}-\mathrm{O})$, $1120(\mathrm{C}-\mathrm{O})$.

M. S. (E.S.I., $\mathrm{m} / \mathrm{z}): 324\left(\mathrm{MNa}^{+}\right)$.

1H RMN $\left(\mathrm{CDCl}_{3}, 300 \mathrm{MHz}\right) \delta(\mathrm{ppm}): 8.26(1 \mathrm{H}, \mathrm{sl}, \mathrm{NH}), 7.09\left(1 \mathrm{H}, \mathrm{d}, \mathrm{J}=8.5 \mathrm{~Hz}, \mathrm{H}_{8}\right)$, $6.54\left(1 \mathrm{H}, \mathrm{dd}, \mathrm{J}=8.5 \mathrm{~Hz}, \mathrm{~J}=2.5 \mathrm{~Hz}, \mathrm{H}_{6}\right), 6.36\left(1 \mathrm{H}, \mathrm{d}, \mathrm{J}=2.5 \mathrm{~Hz}, \mathrm{H}_{5}\right), 5.90(1 \mathrm{H}, \mathrm{d}, \mathrm{J}=$ $\left.10.0 \mathrm{~Hz}, \mathrm{H}_{6}{ }^{\prime}\right), 5.70\left(1 \mathrm{H}, \mathrm{d}, \mathrm{J}=10.0 \mathrm{~Hz}, \mathrm{H}_{7}\right), 4.06-3.90\left(4 \mathrm{H}, \mathrm{m}, \mathrm{H}_{2}\right.$, and $\left.\mathrm{H}_{3}\right), 3.78(3 \mathrm{H}$, s, $\left.\mathrm{OCH}_{3}\right), 2.58\left(2 \mathrm{H}\right.$, system $\mathrm{AB}, \mathrm{J}=16.0 \mathrm{~Hz}, \mathrm{H}_{3 \mathrm{a}}$ and $\left.\mathrm{H}_{3 \mathrm{~b}}\right), 1.77-1.97\left(2 \mathrm{H}, \mathrm{m}, \mathrm{H}_{9}\right.$ ), $1.77-1.67\left(2 \mathrm{H}, \mathrm{m}, \mathrm{H}_{10}\right)$.

${ }^{13} \mathbf{C ~ R M N}\left(\mathrm{CDCl}_{3}, 75 \mathrm{MHz}\right) \delta(\mathrm{ppm}): 170.4\left(\mathrm{C}_{2}\right), 159.7\left(\mathrm{C}_{7}\right), 137.2\left(\mathrm{C}_{4 \mathrm{a}}\right), 136.1\left(\mathrm{C}_{7}\right)$, $130.4\left(\mathrm{C}_{6}\right), 128.3\left(\mathrm{C}_{8}\right), 120.3\left(\mathrm{C}_{8 \mathrm{a}}\right), 108.3\left(\mathrm{C}_{6}\right), 105.0\left(\mathrm{C}_{5}\right), 102.3\left(\mathrm{C}_{5}\right), 64.8$ and 64.7 $\left(\mathrm{C}_{2}\right.$, and $\left.\mathrm{C}_{3}{ }^{\prime}\right), 55.5\left(\mathrm{OCH}_{3}\right), 42.8\left(\mathrm{C}_{3}\right), 38.7\left(\mathrm{C}_{8},\right), 31.8\left(\mathrm{C}_{9},\right), 29.5\left(\mathrm{C}_{10^{\prime}}\right)$.

8-methoxy-3-hydro-1H-quinolin-2-one-4,8'-(1',4'-dioxa-spiro[4.5]dec-6'-enyl) 13c 


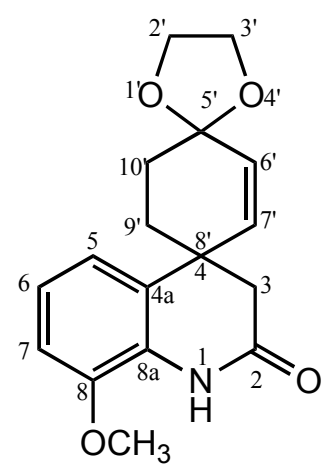

$13 \mathrm{c}$

By Heck conditions : $\mathrm{Pd}_{2}(\mathrm{dba})_{3}(639.7 \mathrm{mg}, 0.70 \mathrm{mmol})$, dppe $(557.2 \mathrm{mg}, 1.40 \mathrm{mmol})$, dry DMA (400 mL), 2-(1',4'-dioxa-spiro[4.5]dec-7'-en-8'-yl)- $N$-(2-iodo-phenyl)acetamide 10c $(6.0 \mathrm{~g}, 13.99 \mathrm{mmol})$, and PMP $(10.1 \mathrm{~mL}, 55.94 \mathrm{mmol})$ were used to produce 13c. Purification: Heptane/EtOAc (4:6). Pale yellow amorphous solid (3.9 g, $94 \%)$.

By Heck “ligand free” conditions : 2-(1',4'-dioxa-spiro[4.5]dec-7'-en-8'-yl)- $N$-(2iodo-6-methoxy-phenyl)-acetamide $10 \mathrm{c}(3.68 \mathrm{~g}, 8.58 \mathrm{mmol}), \mathrm{Pd}_{2}(\mathrm{dba})_{3}(78.5 \mathrm{mg}, 0.09$ mmol), dry DMA $(60 \mathrm{~mL})$, and $\mathrm{NEt}_{3}(3.58 \mathrm{~mL}, 25.73 \mathrm{mmol})$ were used to produce 13c. Purification : Heptane/EtOAc (4:6). Pale yellow amorphous solid (2.21 g, 86\%).

H.R.M.S. (I.C., m/z) calcd for $\mathrm{C}_{17} \mathrm{H}_{19} \mathrm{NNaO}_{4}{ }^{+}\left(\mathrm{MNa}^{+}\right)$324.1206, found : 324.1211 .

I.R. $\left(\mathrm{CHCl}_{3}\right) \vee\left(\mathrm{cm}^{-1}\right)$ : $3404(\mathrm{~N}-\mathrm{H}), 1679(\mathrm{C}=\mathrm{O}$ (lactame)), $1593(\mathrm{C}=\mathrm{C}), 1491$ (C-Car), 1260 (Car-O), 1092 (C-O).

M. S. (I.E., $\mathrm{m} / \mathrm{z}): 301\left(\mathrm{M}^{+}\right.$.).

${ }_{1} \mathbf{H}$ RMN $\left(\mathrm{CDCl}_{3}, 500 \mathrm{MHz}\right) \delta(\mathrm{ppm}): 7.88(1 \mathrm{H}, \mathrm{sl}, \mathrm{NH}), 6.97\left(1 \mathrm{H}, \mathrm{t}, \mathrm{J}=8.1 \mathrm{~Hz}, \mathrm{H}_{6}\right)$, $6.84\left(1 \mathrm{H}, \mathrm{dd}, \mathrm{J}=8.1 \mathrm{~Hz}, \mathrm{~J}=1.1 \mathrm{~Hz}, \mathrm{H}_{7}\right), 6.82\left(1 \mathrm{H}, \mathrm{dd}, \mathrm{J}=8.1 \mathrm{~Hz}, \mathrm{~J}=1.1 \mathrm{~Hz}, \mathrm{H}_{5}\right), 5.90$ $\left(1 \mathrm{H}, \mathrm{d}, \mathrm{J}=10.0 \mathrm{~Hz}, \mathrm{H}_{6^{\prime}}\right), 5.74\left(1 \mathrm{H}, \mathrm{d}, \mathrm{J}=10.0 \mathrm{~Hz}, \mathrm{H}_{7^{\prime}}\right), 4.04\left(4 \mathrm{H}, \mathrm{m}, \mathrm{H}_{2}\right.$, and $\left.\mathrm{H}_{3}{ }^{\prime}\right), 3.87$ $\left(3 \mathrm{H}, \mathrm{s}, \mathrm{OCH}_{3}\right), 2.67-2.54\left(2 \mathrm{H}\right.$, system $\mathrm{AB}, \mathrm{J}=15.0 \mathrm{~Hz}, \mathrm{H}_{3 \mathrm{a}}$ and $\left.\mathrm{H}_{3 \mathrm{~b}}\right), 1.92\left(2 \mathrm{H}, \mathrm{m}, \mathrm{H}_{9}\right)$, $1.76\left(2 \mathrm{H}, \mathrm{m}, \mathrm{H}_{10}\right)$.

${ }^{13} \mathbf{C ~ R M N}\left(\mathrm{CDCl}_{3}, 75 \mathrm{MHz}\right) \delta(\mathrm{ppm}): 168.4\left(\mathrm{C}_{2}\right), 146.1\left(\mathrm{C}_{8}\right), 135.7\left(\mathrm{C}_{7^{\prime}}\right), 130.4\left(\mathrm{C}_{6^{\prime}}\right)$, $128.6\left(\mathrm{C}_{4 \mathrm{a}}\right), 125.3\left(\mathrm{C}_{8 \mathrm{a}}\right), 122.7\left(\mathrm{C}_{6}\right), 119.1\left(\mathrm{C}_{5}\right), 109.6\left(\mathrm{C}_{7}\right), 104.9\left(\mathrm{C}_{5^{\prime}}\right), 64.6\left(\mathrm{C}_{2}\right.$, and $\left.\mathrm{C}_{3}\right), 55.9\left(\mathrm{OCH}_{3}\right), 42.3\left(\mathrm{C}_{3}\right), 39.4\left(\mathrm{C}_{4}\right), 31.4\left(\mathrm{C}_{9^{\prime}}\right), 29.5\left(\mathrm{C}_{10^{\prime}}\right)$.

8-methoxy-3-hydro-1H-quinolin-2-one-4,8'-

(1',4'-dioxa-spiro[4.5]dec-6'-enyl)-carboxylic acid tert-butyl ester 13d 


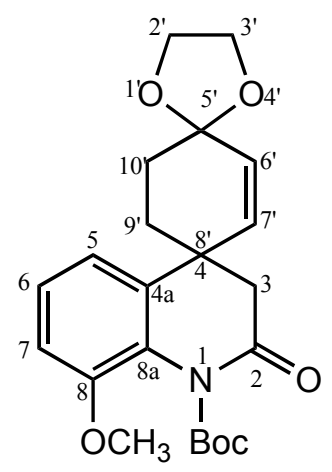

13d

By Heck conditions: $\mathrm{Pd}_{2}(\mathrm{dba})_{3}(45.8 \mathrm{mg}, 0.05 \mathrm{mmol})$, dppe $(39.9 \mathrm{mg}, 0.10 \mathrm{mmol})$, dry DMA (40 mL), (1',4'-dioxaspiro[4.5]dec-7'-en-8'-yl)-acetyl-(2-iodo-6-methoxyphenyl)-carboxylic acid tert-butyl ester 10d (530.2 mg, $1.00 \mathrm{mmol})$, and PMP (723,4 $\mu \mathrm{L}, 4.01 \mathrm{mmol})$ were used to produce $13 \mathbf{d}$ and 13c. Purification : Heptane/EtOAc (4:6). Pale yellow amorphous solid (219.8 $\mathrm{mg}$ and $125.8 \mathrm{mg}, 96 \%$ ).

H.R.M.S. (I.C., m/z) calcd for $\mathrm{C}_{22} \mathrm{H}_{27} \mathrm{NNaO}_{6}{ }^{+}\left(\mathrm{MNa}^{+}\right)$424.1731, found : 424.1710 .

I.R. $\left(\mathrm{CHCl}_{3}\right) \vee\left(\mathrm{cm}^{-1}\right)$ : $1751(\mathrm{C}=\mathrm{O}(\mathrm{Boc})), 1705(\mathrm{C}=\mathrm{O}$ (lactame) $), 1589(\mathrm{C}=\mathrm{C}), 1479$ (C-Car), 1261 (Car-O), 1147 (C-O (Boc)), 1092 (C-O).

M. S. (I.E., $\mathrm{m} / \mathrm{z}): 401\left(\mathrm{M}^{+}\right.$.).

${ }^{1} \mathbf{H}$ RMN $\left(\mathrm{CDCl}_{3}, 300 \mathrm{MHz}\right) \delta(\mathrm{ppm}): 7.10\left(1 \mathrm{H}, \mathrm{t}, \mathrm{J}=8.3 \mathrm{~Hz}, \mathrm{H}_{6}\right), 6.86(1 \mathrm{H}, \mathrm{dd}, \mathrm{J}=$ $\left.8.3 \mathrm{~Hz}, \mathrm{~J}=1.2 \mathrm{~Hz}, \mathrm{H}_{7}\right), 6.85\left(1 \mathrm{H}, \mathrm{dd}, \mathrm{J}=8.3 \mathrm{~Hz}, \mathrm{~J}=1.3 \mathrm{~Hz}, \mathrm{H}_{5}\right), 5.90(1 \mathrm{H}, \mathrm{d}, \mathrm{J}=10.0$ $\left.\mathrm{Hz}, \mathrm{H}_{6^{\prime}}\right), 5.67\left(1 \mathrm{H}, \mathrm{d}, \mathrm{J}=10.0 \mathrm{~Hz}, \mathrm{H}_{7^{\prime}}\right), 4.02\left(4 \mathrm{H}, \mathrm{m}, \mathrm{H}_{2}\right.$, and $\left.\mathrm{H}_{3}{ }^{\prime}\right), 3.83\left(3 \mathrm{H}, \mathrm{s}, \mathrm{OCH}_{3}\right)$, 2.67-2.48 (2H, system $\mathrm{AB}, \mathrm{J}=15.0 \mathrm{~Hz}, \mathrm{H}_{3 \mathrm{a}}$ and $\left.\mathrm{H}_{3 \mathrm{~b}}\right), 1.93\left(2 \mathrm{H}, \mathrm{m}, \mathrm{H}_{9}\right), 1.79(2 \mathrm{H}, \mathrm{m}$, $\left.\mathrm{H}_{10^{\prime}}\right), 1.60$ (9H, s, Boc).

${ }^{13} \mathrm{C}$ RMN $\left(\mathrm{CDCl}_{3}, 75 \mathrm{MHz}\right) \delta(\mathrm{ppm}): 167.9\left(\mathrm{C}_{2}\right), 151.9$ (CO (Boc)), $149.7\left(\mathrm{C}_{8}\right), 135.3$ $\left(\mathrm{C}_{7^{\prime}}\right), 133.9\left(\mathrm{C}_{4 \mathrm{a}}\right), 130.7\left(\mathrm{C}_{6^{\prime}}\right), 126.4\left(\mathrm{C}_{8 \mathrm{a}}\right), 125.5\left(\mathrm{C}_{6}\right), 119.2\left(\mathrm{C}_{5}\right), 111.4\left(\mathrm{C}_{7}\right), 105.0$ $\left(\mathrm{C}_{5^{\prime}}\right), 84.0(\mathrm{C}(\mathrm{Boc})), 64.7\left(\mathrm{C}_{2^{\prime}}\right.$ and $\left.\mathrm{C}_{3^{\prime}}\right), 56.0\left(\mathrm{OCH}_{3}\right), 44.5\left(\mathrm{C}_{3}\right), 39.5\left(\mathrm{C}_{4}\right), 30.8\left(\mathrm{C}_{9^{\prime}}\right)$, $29.6\left(\mathrm{C}_{10^{\prime}}\right), 27.8\left(\mathrm{CH}_{3}(\mathrm{Boc})\right)$.

8-methoxy-1-methyl-3-hydro-1H-quinolin-2-one4,8'-(1',4'-dioxa-spiro[4.5]dec-6'-enyl) $13 \mathbf{e}$ 


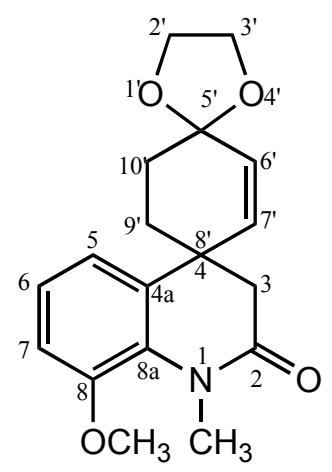

$13 \mathbf{e}$

By Heck conditions : $\mathrm{Pd}_{2}(\mathrm{dba})_{3}(36.8 \mathrm{mg}, 0.04 \mathrm{mmol})$, dppe $(32.1 \mathrm{mg}, 0.08 \mathrm{mmol})$, dry DMA (40 mL), 2-(1',4'-dioxaspiro[4.5]dec-7'-en-8'-yl)- $N$-(2-iodo-6-methoxy-phenyl)$N$-methyl-acetamide 10e (357.0 mg, $0.81 \mathrm{mmol})$, and PMP $(582.0 \mu \mathrm{L}, 3.22 \mathrm{mmol})$ were used to produce 13e. Purification : Heptane/EtOAc (4:6). Pale yellow amorphous solid (203.5 mg, 80\%).

H.R.M.S. (I.C., m/z) calcd for $\mathrm{C}_{18} \mathrm{H}_{22} \mathrm{NO}_{4}{ }^{+}\left(\mathrm{MH}^{+}\right) 316.1543$, found : 316.1563 .

I.R. $\left(\mathrm{CHCl}_{3}\right) \vee\left(\mathrm{cm}^{-1}\right): 1659(\mathrm{C}=\mathrm{O}$ (lactame)), $1589(\mathrm{C}=\mathrm{C}), 1479$ (C-Car), 1261 (CarO), 1092 (C-O).

M. S. (E.S.I., m/z) : $338\left(\mathrm{MNa}^{+}\right), 316\left(\mathrm{MH}^{+}\right)$.

${ }^{1} \mathbf{H}$ RMN $\left(\mathrm{CDCl}_{3}, 300 \mathrm{MHz}\right) \delta(\mathrm{ppm}): 7.05\left(1 \mathrm{H}, \mathrm{t}, \mathrm{J}=8.0 \mathrm{~Hz}, \mathrm{H}_{6}\right), 6.91(1 \mathrm{H}, \mathrm{d}, \mathrm{J}=8.0$ $\left.\mathrm{Hz}, \mathrm{H}_{7}\right), 6.84\left(1 \mathrm{H}, \mathrm{d}, \mathrm{J}=8.3 \mathrm{~Hz}, \mathrm{H}_{5}\right), 5.88\left(1 \mathrm{H}, \mathrm{d}, \mathrm{J}=10.0 \mathrm{~Hz}, \mathrm{H}_{6}\right), 5.65(1 \mathrm{H}, \mathrm{d}, \mathrm{J}=$ $\left.10.0 \mathrm{~Hz}, \mathrm{H}_{7^{\prime}}\right), 4.03\left(4 \mathrm{H}, \mathrm{m}, \mathrm{H}_{2^{\prime}}\right.$ and $\left.\mathrm{H}_{3^{\prime}}\right), 3.86\left(3 \mathrm{H}, \mathrm{s}, \mathrm{OCH}_{3}\right), 3.40\left(3 \mathrm{H}, \mathrm{s}, \mathrm{NCH}_{3}\right), 2.61-$ $2.46\left(2 \mathrm{H}\right.$, system $\mathrm{AB}, \mathrm{J}=15.0 \mathrm{~Hz}, \mathrm{H}_{3 \mathrm{a}}$ and $\left.\mathrm{H}_{3 \mathrm{~b}}\right), 1.91-1.75\left(4 \mathrm{H}, \mathrm{m}, \mathrm{H}_{9^{\prime}}\right.$ and $\left.\mathrm{H}_{10^{\prime}}\right)$.

${ }^{13} \mathbf{C ~ R M N}\left(\mathrm{CDCl}_{3}, 75 \mathrm{MHz}\right) \delta(\mathrm{ppm}): 169.9\left(\mathrm{C}_{2}\right), 150.2\left(\mathrm{C}_{8}\right), 136.0\left(\mathrm{C}_{7}\right), 134.7\left(\mathrm{C}_{4 \mathrm{a}}\right)$, $130.3\left(\mathrm{C}_{6^{\prime}}\right), 129.9\left(\mathrm{C}_{8 \mathrm{a}}\right), 124.6\left(\mathrm{C}_{6}\right), 119.2\left(\mathrm{C}_{5}\right), 112.1\left(\mathrm{C}_{7}\right), 105.1\left(\mathrm{C}_{5^{\prime}}\right), 64.6\left(\mathrm{C}_{2^{\prime}}\right.$ and $\left.\mathrm{C}_{3^{\prime}}\right), 55.9\left(\mathrm{OCH}_{3}\right), 43.7\left(\mathrm{C}_{3}\right), 39.0\left(\mathrm{C}_{4}\right), 34.5\left(\mathrm{NCH}_{3}\right), 30.2\left(\mathrm{C}_{9^{\prime}}\right), 29.7\left(\mathrm{C}_{10^{\prime}}\right)$.

\section{General Procedure for the Synthesis of Carbamate Derivatives 14a-c}

To 13a-c in $\mathrm{THF}$ were added at $0^{\circ} \mathrm{C} \mathrm{HCl} 1 \mathrm{~N}$. After being stirred for a night at room temperature, the reaction mixture was extracted with EtOAc. The combined organic layer was washed with $\mathrm{H}_{2} \mathrm{O}$, brine, dried $\left(\mathrm{MgSO}_{4}\right)$ and evaporated. The residue was dissolved in dry $\mathrm{CH}_{2} \mathrm{Cl}_{2}$. Then $\mathrm{Boc}_{2} \mathrm{O}$, DMAP and $\mathrm{NEt}_{3}$ were added. After being stirred for a night at room temperature, the reaction mixture was extracted with $\mathrm{CH}_{2} \mathrm{Cl}_{2}$. The combined organic layer was washed with $\mathrm{H}_{2} \mathrm{O}$, brine, dried $\left(\mathrm{MgSO}_{4}\right)$ and evaporated. Silica gel flash-column chromatography of the residue afforded 14a-c as indicated below for each case. 
3-hydro-1H-quinolin-2-one-4,1'-(cyclohex-2'-en-4'-one)carboxylic acid tert-butyl ester 14a

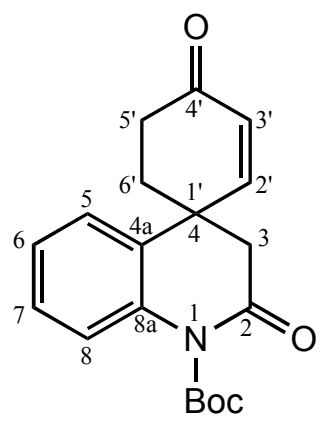

$14 a$

3-hydro-1H-quinolin-2-one-4,8'-(1',4'-dioxa-spiro[4.5]dec-6'-enyl) 13a (4.67 g, 17.23 mmol), THF (400 mL), $\mathrm{HCl} 1 \mathrm{~N}(200 \mathrm{~mL})$, dry $\mathrm{CH}_{2} \mathrm{Cl}_{2}(200 \mathrm{~mL}), \mathrm{Boc}_{2} \mathrm{O}$ (7.52 g, 34.46 mmol), DMAP (526.3 mg, $4.31 \mathrm{mmol}$ ), and $\mathrm{NEt}_{3}(3.6 \mathrm{~mL}, 25.85 \mathrm{mmol}$ ) were used to produce 14a. Purification: Heptane/EtOAc (5:5). White amorphous solid (5.55 g, 98\%).

H.R.M.S. (E.S.I., m/z) calcd for $\mathrm{C}_{19} \mathrm{H}_{21} \mathrm{NNaO}_{4}{ }^{+}\left(\mathrm{MNa}^{+}\right)$350.1358, found : 350.1368 .

I.R. $\left(\mathrm{CHCl}_{3}\right) \vee\left(\mathrm{cm}^{-1}\right): 1743(\mathrm{C}=\mathrm{O}(\mathrm{Boc})), 1674(\mathrm{C}=\mathrm{O}$ (lactame)), $1600(\mathrm{C}=\mathrm{C}), 1492$ (C-Car), 1144 (C-O).

M. S. (E.S.I., $\mathrm{m} / \mathrm{z}): 350\left(\mathrm{MNa}^{+}\right)$.

${ }^{1} \mathbf{H ~ R M N}\left(\mathrm{CDCl}_{3}, 500 \mathrm{MHz}\right) \delta(\mathrm{ppm}): 7.35-7.26\left(1 \mathrm{H}, \mathrm{m}, \mathrm{H}_{6}\right), 7.21-7.13\left(2 \mathrm{H}, \mathrm{m}, \mathrm{H}_{7}\right.$ and $\left.\mathrm{H}_{8}\right), 7.00\left(1 \mathrm{H}, \mathrm{d}, \mathrm{J}=8.2 \mathrm{~Hz}, \mathrm{H}_{5}\right), 6.68\left(1 \mathrm{H}, \mathrm{d}, \mathrm{J}=10.1 \mathrm{~Hz}, \mathrm{H}_{3}\right), 6.28(1 \mathrm{H}, \mathrm{d}, \mathrm{J}=$ $10.1 \mathrm{~Hz}, \mathrm{H}_{2}$ ) $, 2.77\left(2 \mathrm{H}\right.$, system $\mathrm{AB}, \mathrm{J}=15.1 \mathrm{~Hz}, \mathrm{H}_{3 \mathrm{a}}$ and $\left.\mathrm{H}_{3 \mathrm{~b}}\right), 2.51-2.34\left(2 \mathrm{H}, \mathrm{m}, \mathrm{H}_{5}\right.$ ), $2.17\left(2 \mathrm{H}, \mathrm{t}, \mathrm{J}=6.4 \mathrm{~Hz}, \mathrm{H}_{6}\right), 1.61(9 \mathrm{H}, \mathrm{s}, \mathrm{Boc})$.

${ }^{13} \mathrm{C}$ RMN $\left(\mathrm{CDCl}_{3}, 75 \mathrm{MHz}\right) \delta(\mathrm{ppm}): 198.3\left(\mathrm{C}_{4}{ }^{\prime}\right), 166.1\left(\mathrm{C}_{2}\right), 151.3$ (CO (Boc)), 151.3 $\left(\mathrm{C}_{3}\right), 136.1\left(\mathrm{C}_{8 \mathrm{a}}\right), 131.5\left(\mathrm{C}_{2}\right), 128.7\left(\mathrm{C}_{6}\right), 127.9\left(\mathrm{C}_{4 \mathrm{a}}\right), 126.6$ and $124.6\left(\mathrm{C}_{7}\right.$ and $\left.\mathrm{C}_{8}\right)$, $117.8\left(\mathrm{C}_{5}\right), 85.7(\mathrm{C}(\mathrm{Boc})), 42.6\left(\mathrm{C}_{3}\right), 39.6\left(\mathrm{C}_{1}\right), 33.5\left(\mathrm{C}_{5}\right), 32.4\left(\mathrm{C}_{6}\right), 27.7$ $\left(\mathrm{CH}_{3}(\right.$ Boc $\left.)\right)$.

7-methoxy-3-hydro-1H-quinolin-2-one-4,1'-(cyclohex-2'-en-4'-one)carboxylic acid tert-butyl ester $\mathbf{1 4 b}$ 


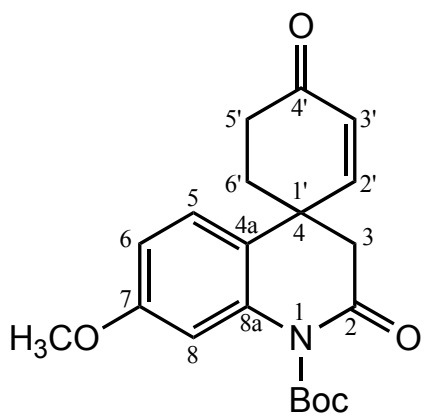

14b

7-methoxy-3-hydro-1H-quinolin-2-one-4,8'-(1',4'-dioxa-spiro[4.5]dec-6'-enyl)

13b

(4.93 g, $16.38 \mathrm{mmol})$, THF (400 mL), $\mathrm{HCl} 1 \mathrm{~N}$ (200 mL), dry $\mathrm{CH}_{2} \mathrm{Cl}_{2}(200 \mathrm{~mL}), \mathrm{Boc}_{2} \mathrm{O}$

(7.15 g, $32.76 \mathrm{mmol})$, DMAP (500.2 mg, $4.09 \mathrm{mmol})$, and $\mathrm{NEt}_{3}(3.4 \mathrm{~mL}, 24.57 \mathrm{mmol})$ were used to produce 14b. Purification : Heptane/EtOAc (5:5). White amorphous solid (5.67 g, 97\%).

H.R.M.S. (E.S.I., m/z) calcd for $\mathrm{C}_{20} \mathrm{H}_{23} \mathrm{NNaO}_{5}{ }^{+}\left(\mathrm{MNa}^{+}\right)$380.1474, found : 380.1448 .

I.R. $\left(\mathrm{CHCl}_{3}\right) \vee\left(\mathrm{cm}^{-1}\right): 1731(\mathrm{C}=\mathrm{O}(\mathrm{Boc})), 1682(\mathrm{C}=\mathrm{O}$ (lactame) $), 1610(\mathrm{C}=\mathrm{C}), 1505$ (C-Car), 1300 (Car-O), 1142 (C-O).

M. S. (E.S.I., $\mathrm{m} / \mathrm{z}): 380\left(\mathrm{MNa}^{+}\right)$.

1H RMN $\left(\mathrm{CDCl}_{3}, 300 \mathrm{MHz}\right) \delta(\mathrm{ppm}): 7.06\left(1 \mathrm{H}, \mathrm{d}, \mathrm{J}=8.5 \mathrm{~Hz}, \mathrm{H}_{5}\right), 6.68-6.63(2 \mathrm{H}, \mathrm{m}$, $\mathrm{H}_{3}$, and $\left.\mathrm{H}_{6}\right), 6.26\left(1 \mathrm{H}, \mathrm{d}, \mathrm{J}=10.1 \mathrm{~Hz}, \mathrm{H}_{2},\right), 3.79\left(3 \mathrm{H}, \mathrm{s}, \mathrm{OCH}_{3}\right), 2.73(2 \mathrm{H}$, system $\mathrm{AB}, \mathrm{J}$ $=15.1 \mathrm{~Hz}, \mathrm{H}_{3 \mathrm{a}}$ and $\left.\mathrm{H}_{3 \mathrm{~b}}\right), 2.48-2.30\left(2 \mathrm{H}, \mathrm{m}, \mathrm{H}_{5},\right), 2.14\left(2 \mathrm{H}, \mathrm{t}, \mathrm{J}=6.6 \mathrm{~Hz}, \mathrm{H}_{6}\right), 1.55(9 \mathrm{H}$, $\mathrm{s}, \mathrm{Boc})$.

${ }^{13} \mathrm{C}$ RMN $\left(\mathrm{CDCl}_{3}, 75 \mathrm{MHz}\right) \delta(\mathrm{ppm}): 198.5\left(\mathrm{C}_{4}{ }^{\prime}\right), 166.3\left(\mathrm{C}_{2}\right), 159.8\left(\mathrm{C}_{7}\right), 151.6\left(\mathrm{C}_{3^{\prime}}\right)$, 151.3 (CO (Boc)), $137.0\left(\mathrm{C}_{8 \mathrm{a}}\right), 131.3\left(\mathrm{C}_{2}\right), 127.6\left(\mathrm{C}_{5}\right), 119.6\left(\mathrm{C}_{4 \mathrm{a}}\right), 109.1\left(\mathrm{C}_{6}\right), 104.3$ $\left(\mathrm{C}_{8}\right), 87.8(\mathrm{C}(\mathrm{Boc})), 55.5\left(\mathrm{OCH}_{3}\right), 42.9\left(\mathrm{C}_{3}\right), 39.1\left(\mathrm{C}_{1}\right), 33.5\left(\mathrm{C}_{5}\right), 32.7\left(\mathrm{C}_{6}\right), 27.6$ $\left(\mathrm{CH}_{3}(\mathrm{Boc})\right)$.

8-methoxy-3-hydro-1H-quinolin-2-one-4,1'-(cyclohex-2'-en-4'-one)carboxylic acid tert-butyl ester 14c 


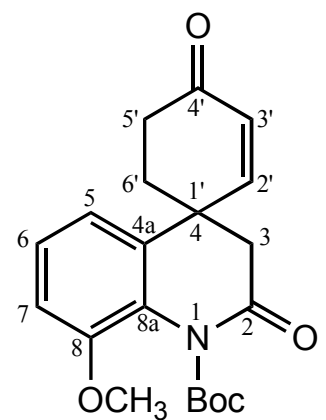

$14 \mathrm{c}$

8-methoxy-3-hydro-1H-quinolin-2-one-4,8'-(1',4'-dioxa-spiro[4.5]dec-6'-enyl)

13c (2.89 g, $9.61 \mathrm{mmol})$, THF (200 mL), $\mathrm{HCl} 1 \mathrm{~N}(100 \mathrm{~mL})$, dry $\mathrm{CH}_{2} \mathrm{Cl}_{2}(120 \mathrm{~mL}), \mathrm{Boc}_{2} \mathrm{O}$ (4.19 g, $19.22 \mathrm{mmol})$, DMAP (293.5 mg, $2.40 \mathrm{mmol})$, and $\mathrm{NEt}_{3}(2.0 \mathrm{~mL}, 14.41 \mathrm{mmol})$ were used to produce 14c. Purification : Heptane/EtOAc (5:5). White amorphous solid (3.30 g, 96\%).

H.R.M.S. (I.C., m/z) calcd for $\mathrm{C}_{20} \mathrm{H}_{23} \mathrm{NNaO}_{5}{ }^{+}\left(\mathrm{MNa}^{+}\right)$380.1468, found : 380.1483 .

I.R. $\left(\mathrm{CHCl}_{3}\right) \vee\left(\mathrm{cm}^{-1}\right): 1751(\mathrm{C}=\mathrm{O}(\mathrm{Boc})), 1696(\mathrm{C}=\mathrm{O}$ (lactame) $), 1590(\mathrm{C}=\mathrm{C}), 1480$ (C-Car), 1261 (Car-O), 1146 (C-O (Boc)).

M. S. (I.E., $\mathrm{m} / \mathrm{z}): 357\left(\mathrm{M}^{+}\right.$.).

${ }^{1} \mathbf{H ~ R M N ~}\left(\mathrm{CDCl}_{3}, 300 \mathrm{MHz}\right) \delta(\mathrm{ppm}): 7.15\left(1 \mathrm{H}, \mathrm{t}, \mathrm{J}=8.1 \mathrm{~Hz}, \mathrm{~J}=7.9 \mathrm{~Hz}, \mathrm{H}_{6}\right), 6.93$ $\left(1 \mathrm{H}, \mathrm{d}, \mathrm{J}=8.3 \mathrm{~Hz}, \mathrm{H}_{7}\right), 6.79\left(1 \mathrm{H}, \mathrm{d}, \mathrm{J}=7.7 \mathrm{~Hz}, \mathrm{H}_{5}\right), 6.67\left(1 \mathrm{H}, \mathrm{d}, \mathrm{J}=10.1 \mathrm{~Hz}, \mathrm{H}_{2}\right), 6.27$ $\left(1 \mathrm{H}, \mathrm{d}, \mathrm{J}=10.1 \mathrm{~Hz}, \mathrm{H}_{3^{\prime}}\right), 3.86\left(3 \mathrm{H}, \mathrm{s}, \mathrm{OCH}_{3}\right), 2.82-2.60(2 \mathrm{H}$, system $\mathrm{AB}, \mathrm{J}=14.4 \mathrm{~Hz}$, $\mathrm{H}_{3 \mathrm{a}}$ and $\left.\mathrm{H}_{3 \mathrm{~b}}\right), 2.44\left(2 \mathrm{H}, \mathrm{m}, \mathrm{H}_{5^{\prime}}\right), 2.19\left(2 \mathrm{H}, \mathrm{m}, \mathrm{H}_{6^{\prime}}\right), 1.60(9 \mathrm{H}, \mathrm{s}, \mathrm{Boc})$.

${ }^{13} \mathbf{C}$ RMN $\left(\mathrm{CDCl}_{3}, 75 \mathrm{MHz}\right) \delta(\mathrm{ppm})$ : $198.4\left(\mathrm{C}_{4^{\prime}}\right), 166.9\left(\mathrm{C}_{2}\right), 151.4\left(\mathrm{C}_{2^{\prime}}\right), 151.3\left(\mathrm{C}_{8}\right)$, 150.0 (CO (Boc)), $131.7\left(\mathrm{C}_{4 \mathrm{a}}\right), 131.3\left(\mathrm{C}_{3^{\prime}}\right), 126.5\left(\mathrm{C}_{8 \mathrm{a}}\right), 125.9\left(\mathrm{C}_{6}\right), 118.3\left(\mathrm{C}_{5}\right), 111.9$ $\left(\mathrm{C}_{7}\right), 84.3(\mathrm{C}(\mathrm{Boc})), 56.0\left(\mathrm{OCH}_{3}\right), 43.6\left(\mathrm{C}_{3}\right), 40.1\left(\mathrm{C}_{4}\right), 33.6\left(\mathrm{C}_{5^{\prime}}\right), 32.0\left(\mathrm{C}_{6^{\prime}}\right), 27.7\left(\mathrm{CH}_{3}\right.$ (Boc)).

\section{General Procedure for the Synthesis of Quinolinone 15a-c}

To 14a-c dissolved in $\mathrm{tBuOH}$ was added $\mathrm{AcOH}$, then $\mathrm{SeO}_{2}$. The reaction mixture was heated at $100^{\circ} \mathrm{C}$ for 72 hours and every 24 hours $\mathrm{SeO}_{2}$ was added. After three days of reaction, the mixture was filtered under celite, washed with EtOAc and evaporated. The residue was dissolved in $\mathrm{CH}_{2} \mathrm{Cl}_{2}$. The combined organic layer was washed with $\mathrm{NaOH} 1 \mathrm{M}, \mathrm{H}_{2} \mathrm{O}$, brine, dried $\left(\mathrm{MgSO}_{4}\right)$ and evaporated. Silica gel flashcolumn chromatography of the residue afforded 15a-c (and 9c) as indicated below for each case. 


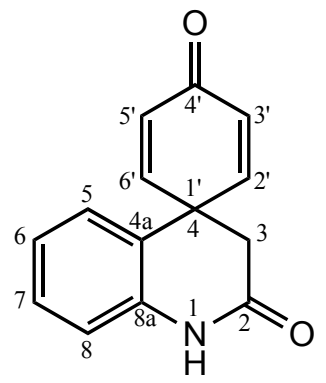

$15 \mathbf{a}$

3-hydro-1H-quinolin-2-one-4,1'-(cyclohex-2'-en-4'-one)-carboxylic acid tert-butyl ester 14a (6.18 g, $18.90 \mathrm{mmol}), \mathrm{tBuOH}(450 \mathrm{~mL}), \mathrm{AcOH}$ (37.8 mL), and $\mathrm{SeO}_{2}(4.19 \mathrm{~g}, 37.80$ mmol) then $(2.10 \mathrm{~g}, 18.90 \mathrm{mmol})$ were used to produce 15a. Purification: Heptane/EtOAc (4:6). Yellow amorphous solid (3.13 g, 74\%).

H.R.M.S. (E.S.I., m/z) calcd for $\mathrm{C}_{14} \mathrm{H}_{11} \mathrm{NNaO}_{2}{ }^{+}\left(\mathrm{MNa}^{+}\right)$248.0687, found : 248.0703.

I.R. $\left(\mathrm{CHCl}_{3}\right) \vee\left(\mathrm{cm}^{-1}\right)$ : $3219(\mathrm{~N}-\mathrm{H}), 1665(\mathrm{C}=\mathrm{O}$ lactame $), 1614(\mathrm{C}=\mathrm{C}), 1462(\mathrm{C}-\mathrm{Car})$.

M. S. (E.S.I., $\mathrm{m} / \mathrm{z}): 248\left(\mathrm{MNa}^{+}\right)$.

1H RMN $\left(\mathrm{CDCl}_{3}, 300 \mathrm{MHz}\right) \delta(\mathrm{ppm}): 9.00(1 \mathrm{H}, \mathrm{sl}, \mathrm{NH}), 7.35-7.28$ (1H, m, Har), 7.13-7.03 (2H, m, Har), $7.02\left(2 \mathrm{H}, \mathrm{d}, \mathrm{J}=\mathrm{J}=10.1, \mathrm{H}_{3}\right.$, and $\mathrm{H}_{5}$ ) $)$ 6.99-6.93 (1H, $\mathrm{m}, \mathrm{Har}$ ), $6.42\left(2 \mathrm{H}, \mathrm{d}, \mathrm{J}=\mathrm{J}=10.1, \mathrm{H}_{2}\right.$, and $\left.\mathrm{H}_{6}\right), 2.80\left(2 \mathrm{H}, \mathrm{s}, \mathrm{H}_{3}\right)$.

${ }^{13} \mathrm{C}$ RMN $\left(\mathrm{CDCl}_{3}, 75 \mathrm{MHz}\right) \delta(\mathrm{ppm}): 184.9\left(\mathrm{C}_{4}\right), 168.2\left(\mathrm{C}_{2}\right), 149.3\left(\mathrm{C}_{3}\right.$, and $\left.\mathrm{C}_{5}{ }\right)$, $136.6\left(\mathrm{C}_{8 \mathrm{a}}\right), 129.8$ (Car), $129.5\left(\mathrm{C}_{2}\right.$, and $\left.\mathrm{C}_{6}\right), 126.8$ (Car), 124.5 (Car), $122.2\left(\mathrm{C}_{4 \mathrm{a}}\right)$, 116.7 (Car), $43.8\left(\mathrm{C}_{1^{\prime}}\right), 40.2\left(\mathrm{C}_{3}\right)$.

7-methoxy-3-hydro-1H-quinolin-2-one-4,1'-(cyclohex-2',5'-en-4'-one) $\mathbf{1 5 b}$<smiles>COc1ccc2c(c1)NC(=O)CC21C=CC(=O)C=C1</smiles>

15b 
7-methoxy-3-hydro-1H-quinolin-2-one-4,1'-(cyclohex-2'-en-4'-one)-carboxylic acid tert-butyl ester 14b (5.0 g, $14.00 \mathrm{mmol})$, tBuOH (350 mL), AcOH (28.0 mL), and $\mathrm{SeO}_{2}$ $(3.11 \mathrm{~g}, 28.01 \mathrm{mmol})$ then $(1.55 \mathrm{~g}, 14.00 \mathrm{mmol})$ were used to produce $\mathbf{1 5 b}$. Purification : Heptane/EtOAc (4:6). Yellow amorphous solid (2.28 g, 64\%).

H.R.M.S. (E.S.I., m/z) calcd for $\mathrm{C}_{15} \mathrm{H}_{13} \mathrm{NNaO}_{3}{ }^{+}\left(\mathrm{MNa}^{+}\right)$278.0793, found : 278.0825 .

I.R. $\left(\mathrm{CHCl}_{3}\right) \vee\left(\mathrm{cm}^{-1}\right): 3209(\mathrm{~N}-\mathrm{H}), 1656(\mathrm{C}=\mathrm{O}$ lactame $), 1613(\mathrm{C}=\mathrm{C}), 1485(\mathrm{C}-\mathrm{Car})$, 1258 (Car-O).

M. S. (E.S.I., $\mathrm{m} / \mathrm{z}): 278\left(\mathrm{MNa}^{+}\right)$.

$\mathbf{1}_{\mathbf{H}} \mathbf{R M N}\left(\mathrm{CDCl}_{3}, 300 \mathrm{MHz}\right) \delta(\mathrm{ppm}): 9.10(1 \mathrm{H}, \mathrm{sl}, \mathrm{NH}), 6.99-6.94\left(3 \mathrm{H}, \mathrm{m}, \mathrm{H}_{5}, \mathrm{H}_{5}\right.$ ، and $\left.\mathrm{H}_{3}{ }^{\prime}\right), 6.57\left(1 \mathrm{H}, \mathrm{dd}, \mathrm{J}=2.5 \mathrm{~Hz}, \mathrm{~J}=8.6 \mathrm{~Hz}, \mathrm{H}_{6}\right), 6.49\left(1 \mathrm{H}, \mathrm{d}, \mathrm{J}=2.5 \mathrm{~Hz}, \mathrm{H}_{8}\right), 6.37$ $\left(2 \mathrm{H}, \mathrm{d}, \mathrm{J}=10.1 \mathrm{~Hz}, \mathrm{H}_{2}\right.$, and $\left.\mathrm{H}_{6}\right), 3.80\left(3 \mathrm{H}, \mathrm{s}, \mathrm{OCH}_{3}\right), 2.75\left(2 \mathrm{H}, \mathrm{s}, \mathrm{H}_{3}\right)$.

${ }^{13} \mathrm{C}$ RMN $\left(\mathrm{CDCl}_{3}, 75 \mathrm{MHz}\right) \delta(\mathrm{ppm}): 185.1\left(\mathrm{C}_{4}\right), 168.6\left(\mathrm{C}_{2}\right), 160.8\left(\mathrm{C}_{7}\right), 149.8\left(\mathrm{C}_{2}\right.$, and $\left.\mathrm{C}_{6}\right), 137.9\left(\mathrm{C}_{8 \mathrm{a}}\right), 129.1\left(\mathrm{C}_{3}\right.$, and $\left.\mathrm{C}_{5}\right), 127.7\left(\mathrm{C}_{5}\right), 114.0\left(\mathrm{C}_{4 \mathrm{a}}\right), 109.6\left(\mathrm{C}_{6}\right), 102.7$ $\left(\mathrm{C}_{8}\right), 55.6\left(\mathrm{OCH}_{3}\right), 43.2\left(\mathrm{C}_{1},\right), 40.2\left(\mathrm{C}_{3}\right)$.

8-methoxy-3-hydro-1H-quinolin-2-one-4,1'-(cyclohex-2',5'-en-4'-one) 15c (and 9c)

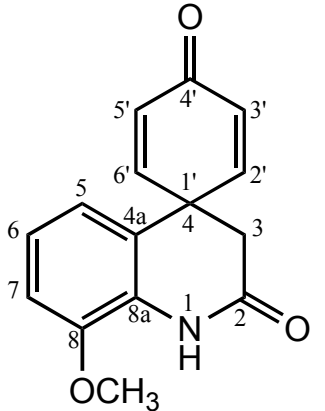

$15 \mathrm{c}$<smiles>COc1cccc2c1N(C(=O)OCc1ccccc1)C(=O)CC21C=CC(=O)C=C1</smiles>

9c

8-methoxy-3-hydro-1H-quinolin-2-one-4,1'-(cyclohex-2'-en-4'-one)-carboxylic acid tert-butyl ester 14c (3.52 g, $9.85 \mathrm{mmol}), \mathrm{tBuOH}(250 \mathrm{~mL}), \mathrm{AcOH}(19.7 \mathrm{~mL})$, and $\mathrm{SeO}_{2}$ $(2.19 \mathrm{~g}, 19.71 \mathrm{mmol})$ then $(1.09 \mathrm{~g}, 9.85 \mathrm{mmol})$ were used to produce $15 \mathrm{c}$ and $9 \mathrm{c}$. Purification: Heptane/EtOAc (4:6). Yellow amorphous solid (604.2 mg and $1.80 \mathrm{~g}$, $74 \%)$.

H.R.M.S. (I.C., m/z) calcd for $\mathrm{C}_{15} \mathrm{H}_{14} \mathrm{NO}_{3}{ }^{+}\left(\mathrm{MH}^{+}\right)$256.0968, found : 256.0997 .

I.R. $\left(\mathrm{CHCl}_{3}\right) \vee\left(\mathrm{cm}^{-1}\right): 3400(\mathrm{~N}-\mathrm{H}), 1669(\mathrm{C}=\mathrm{O}$ (lactame)), $1627(\mathrm{C}=\mathrm{C}), 1493(\mathrm{C}-\mathrm{Car})$, 1263 (Car-O).

M. S. (I.E., $\mathrm{m} / \mathrm{z}): 255\left(\mathrm{M}^{+}\right.$.).

1H RMN $\left(\mathrm{CDCl}_{3}, 300 \mathrm{MHz}\right) \delta(\mathrm{ppm}): 7.94(1 \mathrm{H}, \mathrm{sl}, \mathrm{NH}), 6.92(1 \mathrm{H}, \mathrm{t}, \mathrm{J}=8.1 \mathrm{~Hz}, \mathrm{~J}=$ $\left.7.9 \mathrm{~Hz}, \mathrm{H}_{6}\right), 6.91\left(2 \mathrm{H}, \mathrm{d}, \mathrm{J}=10.1 \mathrm{~Hz}, \mathrm{H}_{2^{\prime}}\right.$ and $\left.\mathrm{H}_{6^{\prime}}\right), 6.81\left(1 \mathrm{H}, \mathrm{d}, \mathrm{J}=8.2 \mathrm{~Hz}, \mathrm{H}_{7}\right), 6.61$ 
$\left(1 \mathrm{H}, \mathrm{d}, \mathrm{J}=7.7 \mathrm{~Hz}, \mathrm{H}_{5}\right), 6.32\left(2 \mathrm{H}, \mathrm{d}, \mathrm{J}=10.1 \mathrm{~Hz}, \mathrm{H}_{3^{\prime}}\right.$ and $\left.\mathrm{H}_{5^{\prime}}\right), 3.84\left(3 \mathrm{H}, \mathrm{s}, \mathrm{OCH}_{3}\right), 2.68$ $\left(2 \mathrm{H}, \mathrm{s}, \mathrm{H}_{3}\right)$.

${ }^{13} \mathbf{C ~ R M N}\left(\mathrm{CDCl}_{3}, 75 \mathrm{MHz}\right) \delta(\mathrm{ppm}): 185.0\left(\mathrm{C}_{4^{\prime}}\right), 166.6\left(\mathrm{C}_{2}\right), 149.3\left(\mathrm{C}_{2^{\prime}}\right.$ and $\left.\mathrm{C}_{6^{\prime}}\right)$, $146.5\left(\mathrm{C}_{8}\right), 129.4\left(\mathrm{C}_{3^{\prime}}\right.$ and $\left.\mathrm{C}_{5^{\prime}}\right), 126.2\left(\mathrm{C}_{4 \mathrm{a}}\right), 123.9\left(\mathrm{C}_{6}\right), 122.3\left(\mathrm{C}_{8 \mathrm{a}}\right), 118.3\left(\mathrm{C}_{5}\right), 110.9$ $\left(\mathrm{C}_{7}\right), 56.1\left(\mathrm{OCH}_{3}\right), 43.9\left(\mathrm{C}_{4}\right), 40.1\left(\mathrm{C}_{3}\right)$.

\section{General Procedure for the Synthesis of Carbamate Derivatives 9a-c}

To a solution of $\mathbf{1 5 a - c}$ in dry $\mathrm{CH}_{2} \mathrm{Cl}_{2}, \mathrm{Boc}_{2} \mathrm{O}$, DMAP and $\mathrm{NEt}_{3}$ was successfully added. After being stirred for a night at room temperature, the reaction mixture was extracted with $\mathrm{CH}_{2} \mathrm{Cl}_{2}$. The combined organic layer was washed with $\mathrm{H}_{2} \mathrm{O}$, brine, dried $\left(\mathrm{MgSO}_{4}\right)$ and evaporated. Silica gel flash-column chromatography of the residue afforded 9a-c as indicated below for each case.

3-hydro-1H-quinolin-2-one-4,1'-(cyclohex-2',5'-en-4'-one)carboxylic acid tert-butyl ester 9a<smiles>O=C1C=CC2(C=C1)CC(=O)N(C(=O)OCc1ccccc1)c1ccccc12</smiles>

9a

3-hydro-1H-quinolin-2-one-4,1'-(cyclohex-2',5'-en-4'-one) 15a (1.55 g, 6.89 mmol), dry $\mathrm{CH}_{2} \mathrm{Cl}_{2}(85 \mathrm{~mL}), \mathrm{Boc}_{2} \mathrm{O}(3.0 \mathrm{~g}, 13.78 \mathrm{mmol})$, DMAP (210.4 mg, $\left.1.72 \mathrm{mmol}\right)$, and $\mathrm{NEt}_{3}$ $(1.44 \mathrm{~mL}, 10.33 \mathrm{mmol})$ were used to produce 9a. Purification : Heptane/EtOAc (6:4). Yellow amorphous solid (1.90 g, 85\%).

H.R.M.S. (E.S.I., m/z) calcd for $\mathrm{C}_{14} \mathrm{H}_{11} \mathrm{NNaO}_{2}{ }^{+}\left(\mathrm{MNa}^{+}\right)$348.1212, found : 348.1183 . I.R. $\left(\mathrm{CHCl}_{3}\right) \vee\left(\mathrm{cm}^{-1}\right)$ : $1758(\mathrm{C}=\mathrm{O}(\mathrm{Boc})), 1693(\mathrm{C}=\mathrm{O}$ (lactame) $), 1629(\mathrm{C}=\mathrm{C}), 1493$ (C-Car), 1142 (C-O).

M. S. (E.S.I., $\mathrm{m} / \mathrm{z}): 348\left(\mathrm{MNa}^{+}\right)$.

1H RMN $\left(\mathrm{CDCl}_{3}, 300 \mathrm{MHz}\right) \delta(\mathrm{ppm}):$ : 7.40-7.28 (1H, m, Har), 7.13-7.09 (2H, m, Har), $7.02(1 \mathrm{H}, \mathrm{m}, \mathrm{Har}), 6.96\left(2 \mathrm{H}, \mathrm{d}, \mathrm{J}=10.1 \mathrm{~Hz}, \mathrm{H}_{2}\right.$, and $\left.\mathrm{H}_{6}\right), 6.41(2 \mathrm{H}, \mathrm{d}, \mathrm{J}=10.1$ $\mathrm{Hz}, \mathrm{H}_{3}$, and $\mathrm{H}_{5}$ ) $, 2.80\left(2 \mathrm{H}, \mathrm{s}, \mathrm{H}_{3}\right), 1.64(9 \mathrm{H}, \mathrm{s}, \mathrm{Boc})$. 
${ }^{13} \mathrm{C} \mathrm{RMN}\left(\mathrm{CDCl}_{3}, 75 \mathrm{MHz}\right) \delta(\mathrm{ppm})$ : $184.9\left(\mathrm{C}_{4}\right), 165.4\left(\mathrm{C}_{2}\right), 151.4$ (CO (Boc)), 148.7 $\left(\mathrm{C}_{2},\right), 136.6\left(\mathrm{C}_{8 \mathrm{a}}\right), 129.9\left(\mathrm{C}_{3^{\prime}}\right), 129.5$ (Car), 126.7 (Car), 125.3 (Car), $124.4\left(\mathrm{C}_{4 \mathrm{a}}\right), 117.7$ (Car), 86.2 (C (Boc)), $43.4\left(\mathrm{C}_{1}\right), 41.3\left(\mathrm{C}_{3}\right), 27.8\left(\mathrm{CH}_{3}(\mathrm{Boc})\right)$.

\section{7-methoxy-3-hydro-1H-quinolin-2-one-4,1'-(cyclohex-2',5'-en-4'-one)- carboxylic acid tert-butyl ester $\mathbf{9 b}$}

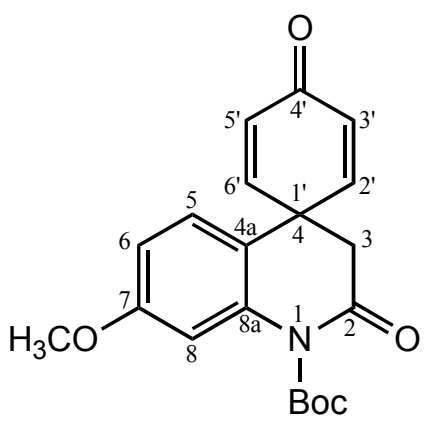

9b

7-methoxy-3-hydro-1H-quinolin-2-one-4,1'-(cyclohex-2',5'-en-4'-one) $\mathbf{1 5 b}$ (1.91 g, 7.48 mmol), dry $\mathrm{CH}_{2} \mathrm{Cl}_{2}(95 \mathrm{~mL}), \mathrm{Boc}_{2} \mathrm{O}$ (3.27 g, $\left.14.96 \mathrm{mmol}\right)$, DMAP (228.5 mg, 1.87 mmol), and $\mathrm{NEt}_{3}(1.56 \mathrm{~mL}, 11.22 \mathrm{mmol})$ were used to produce 9b. Purification : Heptane/EtOAc (6:4). Yellow amorphous solid (2.47 g, 93\%).

H.R.M.S. (E.S.I., m/z) calcd for $\mathrm{C}_{20} \mathrm{H}_{21} \mathrm{NNaO}_{5}{ }^{+}\left(\mathrm{MNa}^{+}\right)$378.1317, found : 378.1303 .

I.R. $\left(\mathrm{CHCl}_{3}\right) \vee\left(\mathrm{cm}^{-1}\right): 1764(\mathrm{C}=\mathrm{O}(\mathrm{Boc})), 1697(\mathrm{C}=\mathrm{O}$ (lactame)), $1667(\mathrm{C}=\mathrm{C}), 1507$ (C-Car), 1305 (Car-O), 1143 (C-O).

M. S. (E.S.I., $\mathrm{m} / \mathrm{z}): 378\left(\mathrm{MNa}^{+}\right)$.

1H RMN $\left(\mathrm{CDCl}_{3}, 300 \mathrm{MHz}\right) \delta(\mathrm{ppm}): 6.99\left(1 \mathrm{H}, \mathrm{d}, \mathrm{J}=8.5 \mathrm{~Hz}, \mathrm{H}_{5}\right), 6.93(2 \mathrm{H}, \mathrm{d}, \mathrm{J}=9.9$ $\mathrm{Hz}, \mathrm{H}_{2}$, and $\left.\mathrm{H}_{6}{ }^{\prime}\right), 6.63\left(1 \mathrm{H}, \mathrm{dd}, \mathrm{J}=2.3 \mathrm{~Hz}, \mathrm{~J}=8.5 \mathrm{~Hz}, \mathrm{H}_{6}\right), 6.57\left(1 \mathrm{H}, \mathrm{d}, \mathrm{J}=2.4 \mathrm{~Hz}, \mathrm{H}_{8}\right)$, $6.38\left(2 \mathrm{H}, \mathrm{d}, \mathrm{J}=9.9 \mathrm{~Hz}, \mathrm{H}_{3}\right.$, and $\left.\mathrm{H}_{5}\right), 3.78\left(3 \mathrm{H}, \mathrm{s}, \mathrm{OCH}_{3}\right), 2.77\left(2 \mathrm{H}, \mathrm{s}, \mathrm{H}_{3}\right), 1.64(9 \mathrm{H}, \mathrm{s}$, Boc).

${ }^{13} \mathbf{C}$ RMN $\left(\mathrm{CDCl}_{3}, 75 \mathrm{MHz}\right) \delta(\mathrm{ppm})$ : $184.9\left(\mathrm{C}_{4}{ }^{\prime}\right), 165.6\left(\mathrm{C}_{2}\right), 160.4\left(\mathrm{C}_{7}\right), 151.0(\mathrm{CO}$ (Boc)), $149.2\left(\mathrm{C}_{2}\right.$, and $\left.\mathrm{C}_{6}\right), 137.5\left(\mathrm{C}_{8 \mathrm{a}}\right), 129.5\left(\mathrm{C}_{3}\right.$, and $\left.\mathrm{C}_{5}\right), 127.6\left(\mathrm{C}_{5}\right), 116.0\left(\mathrm{C}_{4 \mathrm{a}}\right)$, $110.0\left(\mathrm{C}_{6}\right), 104.0\left(\mathrm{C}_{8}\right), 86.2(\mathrm{C}(\mathrm{Boc})), 55.6\left(\mathrm{OCH}_{3}\right), 42.9\left(\mathrm{C}_{4}\right), 41.3\left(\mathrm{C}_{3}\right), 27.7$ $\left(\mathrm{CH}_{3}(\mathrm{Boc})\right)$. 


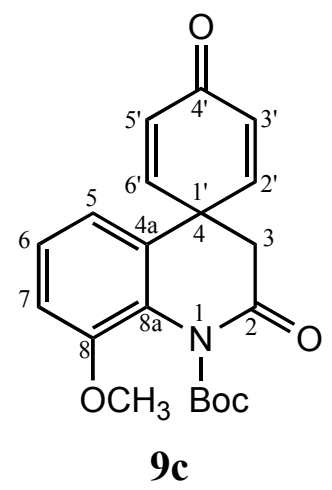

8-methoxy-3-hydro-1H-quinolin-2-one-4,1'-(cyclohex-2',5'-en-4'-one) 15c (1.16 g, 4.55 mmol), dry $\mathrm{CH}_{2} \mathrm{Cl}_{2}(90 \mathrm{~mL}), \mathrm{Boc}_{2} \mathrm{O}$ (1.98 g, $\left.9.10 \mathrm{mmol}\right)$, DMAP (138.9 mg, 1.13 mmol), and $\mathrm{NEt}_{3}(0.95 \mathrm{~mL}, 6.82 \mathrm{mmol})$ were used to produce 9c. Purification: Heptane/EtOAc (6:4). Yellow amorphous solid (1.29 g, 81\%).

H.R.M.S. (I.C., m/z) calcd for $\mathrm{C}_{20} \mathrm{H}_{21} \mathrm{NNaO}_{5}{ }^{+}\left(\mathrm{MNa}^{+}\right)$378.1312, found : 378.1320 .

I.R. $\left(\mathrm{CHCl}_{3}\right) \vee\left(\mathrm{cm}^{-1}\right)$ : $1753(\mathrm{C}=\mathrm{O}(\mathrm{Boc})), 1706(\mathrm{C}=\mathrm{O}$ (lactame) $), 1628(\mathrm{C}=\mathrm{C}), 1480$ (C-Car), 1261 (Car-O), 1146 (C-O (Boc)).

M. S. (I.E., $\mathrm{m} / \mathrm{z}): 355\left(\mathrm{M}^{+}\right.$.).

1H RMN $\left(\mathrm{CDCl}_{3}, 500 \mathrm{MHz}\right) \delta(\mathrm{ppm}): 7.12\left(1 \mathrm{H}, \mathrm{t}, \mathrm{J}=8.1 \mathrm{~Hz}, \mathrm{~J}=7.9 \mathrm{~Hz}, \mathrm{H}_{6}\right), 6.93$ $\left(1 \mathrm{H}, \mathrm{d}, \mathrm{J}=8.3 \mathrm{~Hz}, \mathrm{H}_{7}\right), 6.91\left(2 \mathrm{H}, \mathrm{d}, \mathrm{J}=10.1 \mathrm{~Hz}, \mathrm{H}_{2^{\prime}}\right.$ and $\left.\mathrm{H}_{6^{\prime}}\right), 6.72(1 \mathrm{H}, \mathrm{d}, \mathrm{J}=7.9 \mathrm{~Hz}$, $\left.\mathrm{H}_{5}\right), 6.43\left(2 \mathrm{H}, \mathrm{d}, \mathrm{J}=10.1 \mathrm{~Hz}, \mathrm{H}_{3^{\prime}}\right.$ and $\left.\mathrm{H}_{5^{\prime}}\right), 3.86\left(3 \mathrm{H}, \mathrm{s}, \mathrm{OCH}_{3}\right), 2.75\left(2 \mathrm{H}, \mathrm{s}, \mathrm{H}_{3}\right), 1.61$ $(9 \mathrm{H}, \mathrm{s}, \mathrm{Boc})$.

${ }^{13} \mathbf{C ~ R M N}\left(\mathrm{CDCl}_{3}, 75 \mathrm{MHz}\right) \delta(\mathrm{ppm}): 184.8\left(\mathrm{C}_{4^{\prime}}\right), 166.2\left(\mathrm{C}_{2}\right), 151.1$ (CO (Boc)), 149.9 $\left(\mathrm{C}_{8}\right), 148.5\left(\mathrm{C}_{2^{\prime}}\right.$ and $\left.\mathrm{C}_{6^{\prime}}\right), 129.9\left(\mathrm{C}_{3^{\prime}}\right.$ and $\left.\mathrm{C}_{5^{\prime}}\right), 128.4\left(\mathrm{C}_{4 \mathrm{a}}\right), 127.0\left(\mathrm{C}_{8 \mathrm{a}}\right), 126.5\left(\mathrm{C}_{6}\right), 118.3$ $\left(\mathrm{C}_{5}\right), 112.5\left(\mathrm{C}_{7}\right), 84.7(\mathrm{C}(\mathrm{Boc})), 56.0\left(\mathrm{OCH}_{3}\right), 43.7\left(\mathrm{C}_{4}\right), 42.5\left(\mathrm{C}_{3}\right), 27.7\left(\mathrm{CH}_{3}(\mathrm{Boc})\right)$.

\section{General Procedure for the Synthesis of Carbazole 17a-c}

To 9a-c $\mathrm{MeNH}_{2}$ (33\% in ethanol) was added. After being stirred for $45 \mathrm{~min}$., the solvent was evaporated. The residue was dissolved in a mixture of THF and $\mathrm{NaOH}$ $1 \mathrm{M}(1 / 1)$. After being stirred for 2 hours, the reaction mixture was extracted with $\mathrm{CH}_{2} \mathrm{Cl}_{2}$. The combined organic layer was washed with $\mathrm{H}_{2} \mathrm{O}$, brine, dried $\left(\mathrm{MgSO}_{4}\right)$ and evaporated. Silica gel flash-column chromatography of the residue afforded $\mathbf{1 7 a - c}$ as indicated below for each case.

( \pm )-3-methyl-2,5-dioxo-1,3a,4,6,6a-pentahydro-pyrrolo[2,3- $d]$ carbazole7-carboxylic acid tert-butyl ester 17a 


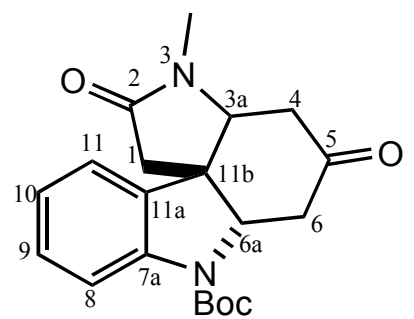

$17 \mathbf{a}$

3-hydro-1H-quinolin-2-one-4,1'-(cyclohex-2',5'-en-4'-one)-carboxylic acid tert-butyl ester 9a (500.0 mg, $1.54 \mathrm{mmol}), \mathrm{MeNH}_{2}(33 \%$ in ethanol) $(60 \mathrm{~mL})$, THF $(12 \mathrm{~mL})$, $\mathrm{NaOH} 1 \mathrm{M}(12 \mathrm{~mL})$ were used to produce 17a. Purification : $\mathrm{CH}_{2} \mathrm{Cl}_{2} / \mathrm{MeOH}(9.9: 0.1)$. Yellow amorphous solid (373.7 mg, 68\%).

H.R.M.S. (E.S.I., m/z) calcd for $\mathrm{C}_{20} \mathrm{H}_{24} \mathrm{~N}_{2} \mathrm{NaO}_{4}{ }^{+}\left(\mathrm{MNa}^{+}\right)$379.1634, found : 379.1613 .

I.R. $\left(\mathrm{CHCl}_{3}\right) \vee\left(\mathrm{cm}^{-1}\right): 1686(\mathrm{C}=\mathrm{O}), 1479$ (C-Car), 1254 (Car-O), 1147 (C-O).

M. S. (E.S.I., $\mathrm{m} / \mathrm{z}): 379\left(\mathrm{MNa}^{+}\right)$.

1H RMN $\left(\mathrm{CDCl}_{3}, 300 \mathrm{MHz}\right) \delta(\mathrm{ppm}): 7.75\left(1 \mathrm{H}, \mathrm{d}, \mathrm{J}=7.9 \mathrm{~Hz}, \mathrm{H}_{8}\right), 7.30(1 \mathrm{H}, \mathrm{dd}, \mathrm{J}=$ $\left.7.4 \mathrm{~Hz}, \mathrm{~J}=1.4 \mathrm{~Hz}, \mathrm{H}_{9}\right), 7.20\left(1 \mathrm{H}, \mathrm{dd}, \mathrm{J}=7.2 \mathrm{~Hz}, \mathrm{~J}=1.4 \mathrm{~Hz}, \mathrm{H}_{11}\right), 7.06(1 \mathrm{H}, \mathrm{dd}, \mathrm{J}=7.2$ $\left.\mathrm{Hz}, \mathrm{J}=1.4 \mathrm{~Hz}, \mathrm{H}_{10}\right), 4.47\left(1 \mathrm{H}, \mathrm{dd}, \mathrm{J}=7.2 \mathrm{~Hz}, \mathrm{~J}=5.3 \mathrm{~Hz}, \mathrm{H}_{6 \mathrm{a}}\right), 3.97(1 \mathrm{H}, \mathrm{dd}, \mathrm{J}=7.5$ $\left.\mathrm{Hz}, \mathrm{J}=3.8 \mathrm{~Hz}, \mathrm{H}_{3 \mathrm{a}}\right), 2.90\left(3 \mathrm{H}, \mathrm{s}, \mathrm{NCH}_{3}\right), 2.86-2.74\left(4 \mathrm{H}, \mathrm{m}, \mathrm{H}_{1}\right.$ and $\left.\mathrm{H}_{6}\right), 2.68(1 \mathrm{H}, \mathrm{dd}, \mathrm{J}$ $\left.=3.8 \mathrm{~Hz}, \mathrm{~J}=17.2 \mathrm{~Hz}, \mathrm{H}_{4}\right), 2.49\left(1 \mathrm{H}, \mathrm{dd}, \mathrm{J}=7.5 \mathrm{~Hz}, \mathrm{~J}=17.2 \mathrm{~Hz}, \mathrm{H}_{4}\right), 1.57(9 \mathrm{H}, \mathrm{s}$, Boc).

${ }^{13} \mathbf{C ~ R M N}\left(\mathrm{CDCl}_{3}, 75 \mathrm{MHz}\right) \delta(\mathrm{ppm}): 205.2\left(\mathrm{C}_{5}\right), 172.2\left(\mathrm{C}_{2}\right), 151.9(\mathrm{CO}(\mathrm{Boc})), 141.3$ $\left(\mathrm{C}_{7 \mathrm{a}}\right), 133.3\left(\mathrm{C}_{11 \mathrm{a}}\right), 129.7\left(\mathrm{C}_{9}\right), 123.8\left(\mathrm{C}_{10}\right), 122.2\left(\mathrm{C}_{11}\right), 115.9\left(\mathrm{C}_{8}\right), 82.6(\mathrm{C}(\mathrm{Boc})), 65.1$ $\left(\mathrm{C}_{6 \mathrm{a}}\right), 63.9\left(\mathrm{C}_{3 \mathrm{a}}\right), 47.0\left(\mathrm{C}_{11 \mathrm{~b}}\right), 44.9\left(\mathrm{C}_{4}\right), 41.1$ and $39.7\left(\mathrm{C}_{1}\right.$ and $\left.\mathrm{C}_{6}\right), 28.4\left(\mathrm{CH}_{3}(\mathrm{Boc})\right)$, $27.6\left(\mathrm{NCH}_{3}\right)$.

( \pm )-9-methoxy-3-methyl-2,5-dioxo-1,3a,4,6,6a-pentahydro-pyrrolo[2,3- $d$ ]carbazole7-carboxylic acid tert-butyl ester $\mathbf{1 7 b}$

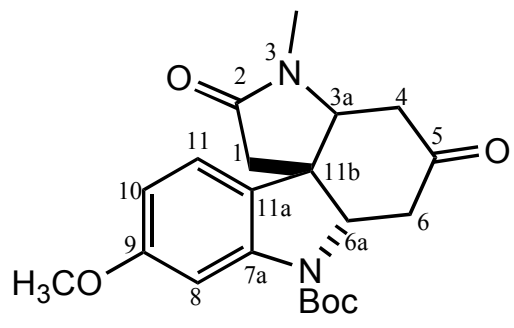

$17 \mathbf{b}$ 
7-methoxy-3-hydro-1H-quinolin-2-one-4,1'-(cyclohex-2',5'-en-4'-one)-carboxylic acid tert-butyl ester $9 \mathbf{b}$ (408.7 mg, $1.15 \mathrm{mmol}), \mathrm{MeNH}_{2}$ (33\% in ethanol) (45 mL), THF (8 $\mathrm{mL}), \quad \mathrm{NaOH} \quad 1 \mathrm{M} \quad(8 \mathrm{~mL})$ were used to produce 17b. Purification : $\mathrm{CH}_{2} \mathrm{Cl}_{2} / \mathrm{MeOH}$ (9.9:0.1). Yellow amorphous solid (293.7 mg, 66\%).

H.R.M.S. (E.S.I., m/z) calcd for $\mathrm{C}_{21} \mathrm{H}_{26} \mathrm{~N}_{2} \mathrm{NaO}_{5}{ }^{+}\left(\mathrm{MNa}^{+}\right)$409.1739, found : 409.1726 .

I.R. $\left(\mathrm{CHCl}_{3}\right) \vee\left(\mathrm{cm}^{-1}\right): 1692(\mathrm{C}=\mathrm{O}), 1497$ (C-Car), 1253 (Car-O), $1162(\mathrm{C}-\mathrm{O})$.

M. S. (E.S.I., $\mathrm{m} / \mathrm{z}): 409\left(\mathrm{MNa}^{+}\right)$.

${ }^{1} \mathbf{H}$ RMN $\left(\mathrm{CDCl}_{3}, 300 \mathrm{MHz}\right) \delta(\mathrm{ppm}): 7.37\left(1 \mathrm{H}, \mathrm{sl}, \mathrm{H}_{8}\right), 7.05\left(1 \mathrm{H}, \mathrm{d}, \mathrm{J}=8.3 \mathrm{~Hz}, \mathrm{H}_{11}\right)$, $6.58\left(1 \mathrm{H}, \mathrm{dd}, \mathrm{J}=8.3 \mathrm{~Hz}, \mathrm{~J}=2.4 \mathrm{~Hz}, \mathrm{H}_{10}\right), 4.44\left(1 \mathrm{H}, \mathrm{t}, \mathrm{J}=6.0 \mathrm{~Hz}, \mathrm{H}_{6 \mathrm{a}}\right), 3.90(1 \mathrm{H}, \mathrm{dd}, \mathrm{J}$ $\left.=3.7 \mathrm{~Hz}, \mathrm{~J}=7.2 \mathrm{~Hz}, \mathrm{H}_{3 \mathrm{a}}\right), 3.79\left(3 \mathrm{H}, \mathrm{s}, \mathrm{OCH}_{3}\right), 2.86\left(3 \mathrm{H}, \mathrm{s}, \mathrm{NCH}_{3}\right), 2.79-2.69(4 \mathrm{H}, \mathrm{m}$, $\mathrm{H}_{1}$ and $\left.\mathrm{H}_{6}\right), 2.63\left(1 \mathrm{H}, \mathrm{dd}, \mathrm{J}=3.7 \mathrm{~Hz}, \mathrm{~J}=17.2 \mathrm{~Hz}, \mathrm{H}_{4}\right), 2.46(1 \mathrm{H}, \mathrm{dd}, \mathrm{J}=7.3 \mathrm{~Hz}, \mathrm{~J}=$ $\left.17.2 \mathrm{~Hz}, \mathrm{H}_{4}\right), 1.55$ (9H, s, Boc).

${ }^{13} \mathrm{C}$ RMN $\left(\mathrm{CDCl}_{3}, 75 \mathrm{MHz}\right) \delta(\mathrm{ppm})$ : $205.4\left(\mathrm{C}_{5}\right), 172.3\left(\mathrm{C}_{2}\right), 161.1\left(\mathrm{C}_{9}\right), 151.8(\mathrm{CO}$ (Boc)), $142.7\left(\mathrm{C}_{7 \mathrm{a}}\right), 125.0\left(\mathrm{C}_{11 \mathrm{a}}\right), 122.7\left(\mathrm{C}_{11}\right), 109.9\left(\mathrm{C}_{10}\right), 101.8\left(\mathrm{C}_{8}\right), 82.6(\mathrm{C}(\mathrm{Boc}))$, $65.8\left(\mathrm{C}_{6 \mathrm{a}}\right), 64.1\left(\mathrm{C}_{3 \mathrm{a}}\right), 55.6\left(\mathrm{OCH}_{3}\right), 46.5\left(\mathrm{C}_{11 \mathrm{~b}}\right), 45.0\left(\mathrm{C}_{6}\right), 41.2\left(\mathrm{C}_{1}\right), 39.6\left(\mathrm{C}_{4}\right), 28.4$ $\left(\mathrm{CH}_{3}(\mathrm{Boc})\right), 27.6\left(\mathrm{NCH}_{3}\right)$.

( \pm )-8-methoxy-3-methyl-2,5-dioxo-1,3a,4,6,6a-pentahydro-pyrrolo[2,3- $d]$ carbazole7-carboxylic acid tert-butyl ester 17c

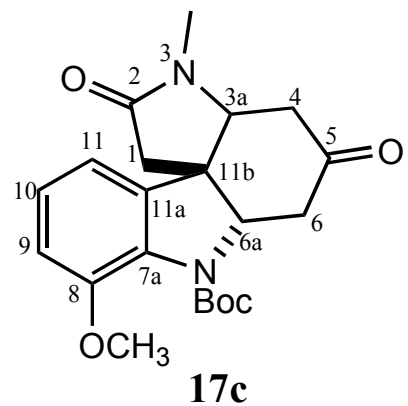

8-methoxy-3-hydro-1H-quinolin-2-one-4,1'-(cyclohex-2',5'-en-4'-one)-carboxylic acid tert-butyl ester 9c (297.7 mg, $0.84 \mathrm{mmol}), \mathrm{MeNH}_{2}$ (33\% in ethanol) (35 mL), THF (7 $\mathrm{mL}), \quad \mathrm{NaOH} \quad 1 \mathrm{M}(7 \mathrm{~mL})$ were used to produce 17c. Purification : $\mathrm{CH}_{2} \mathrm{Cl}_{2} / \mathrm{MeOH}$ (9.9:0.1). Yellow amorphous solid (240.5 mg, 74\%).

H.R.M.S. (I.C., m/z) calcd for $\mathrm{C}_{21} \mathrm{H}_{26} \mathrm{~N}_{2} \mathrm{NaO}_{5}{ }^{+}\left(\mathrm{MNa}^{+}\right)$409.1734, found : 409.1728 .

I.R. $\left(\mathrm{CHCl}_{3}\right) \vee\left(\mathrm{cm}^{-1}\right): 1706(\mathrm{C}=\mathrm{O}(\mathrm{Boc})), 1692(\mathrm{C}=\mathrm{O}$ (lactame)), 1491 (C-Car), 1261 (Car-O), 1161 (C-O (Boc)).

M. S. (I.E., $\mathrm{m} / \mathrm{z}): 386\left(\mathrm{M}^{+} \cdot\right)$. 
1H RMN $\left(\mathrm{CDCl}_{3}, 300 \mathrm{MHz}\right) \delta(\mathrm{ppm}): 7.13\left(1 \mathrm{H}, \mathrm{t}, \mathrm{J}=7.9 \mathrm{~Hz}, \mathrm{~J}=7.7 \mathrm{~Hz}, \mathrm{H}_{10}\right), 6.90$ $\left(1 \mathrm{H}, \mathrm{d}, \mathrm{J}=8.1 \mathrm{~Hz}, \mathrm{H}_{9}\right), 6.78\left(1 \mathrm{H}, \mathrm{d}, \mathrm{J}=7.5 \mathrm{~Hz}, \mathrm{H}_{11}\right), 4.68(1 \mathrm{H}, \mathrm{dd}, \mathrm{J}=5.1 \mathrm{~Hz}, \mathrm{~J}=11.4$ $\left.\mathrm{Hz}, \mathrm{H}_{6 \mathrm{a}}\right), 4.02\left(1 \mathrm{H}, \mathrm{dd}, \mathrm{J}=9.2 \mathrm{~Hz}, \mathrm{~J}=4.0 \mathrm{~Hz}, \mathrm{H}_{3 \mathrm{a}}\right), 3.87\left(3 \mathrm{H}, \mathrm{s}, \mathrm{OCH}_{3}\right), 2.94(3 \mathrm{H}, \mathrm{s}$, $\left.\mathrm{NCH}_{3}\right), 2.80\left(1 \mathrm{H}, \mathrm{d}, \mathrm{J}=16.7 \mathrm{~Hz}, \mathrm{H}_{1}\right), 2.78\left(2 \mathrm{H}, \mathrm{m}, \mathrm{H}_{4}\right.$ and $\left.\mathrm{H}_{6}\right), 2.64(1 \mathrm{H}, \mathrm{d}, \mathrm{J}=16.5$ $\left.\mathrm{Hz}, \mathrm{H}_{1}\right), 2.40\left(2 \mathrm{H}, \mathrm{dd}, \mathrm{J}=10.2 \mathrm{~Hz}, \mathrm{~J}=16.4 \mathrm{~Hz}, \mathrm{H}_{4}\right.$ and $\left.\mathrm{H}_{6}\right), 1.50(9 \mathrm{H}, \mathrm{s}, \mathrm{Boc})$.

${ }^{13} \mathbf{C ~ R M N}\left(\mathrm{CDCl}_{3}, 75 \mathrm{MHz}\right) \delta(\mathrm{ppm}): 205.1\left(\mathrm{C}_{5}\right), 171.7\left(\mathrm{C}_{2}\right), 153.0$ (CO (Boc)), 150.5 $\left(\mathrm{C}_{8}\right), 138.7\left(\mathrm{C}_{11 \mathrm{a}}\right), 129.0\left(\mathrm{C}_{7 \mathrm{a}}\right), 126.9\left(\mathrm{C}_{10}\right), 113.6\left(\mathrm{C}_{11}\right), 112.9\left(\mathrm{C}_{9}\right), 81.5(\mathrm{C}(\mathrm{Boc})), 67.2$ $\left(\mathrm{C}_{6 \mathrm{a}}\right), 62.0\left(\mathrm{C}_{3 \mathrm{a}}\right), 55.6\left(\mathrm{OCH}_{3}\right), 48.5\left(\mathrm{C}_{11 \mathrm{~b}}\right), 43.9\left(\mathrm{C}_{1}\right), 42.8\left(\mathrm{C}_{6}\right), 40.9\left(\mathrm{C}_{4}\right), 28.1\left(\mathrm{CH}_{3}\right.$ (Boc)), $27.9\left(\mathrm{NCH}_{3}\right)$.

\section{General Procedure for the Synthesis of Carbazole 18a-c or 19a-c}

To 9a-c the corresponding amine $\left(\mathrm{EtNH}_{2}\right.$ or $\left.\mathrm{BuNH}_{2}\right)$ was added. After being stirred for $1 \mathrm{~h} 30$ at room temperature, the solvent was evaporated. The residue was purified by preparative TLC plates to afford a fraction of the desired product, 18a-c or 19a-c, and another fraction of the "intermediate open" 16a-c, which was engaged thereafter. The "intermediate open" was dissolved in dry THF, and $\mathrm{NaH}(60 \%)$ was added at $\mathrm{O}^{\circ} \mathrm{C}$. After being stirred for $1 \mathrm{~h} 30$ at room temperature, the reaction mixture was extracted with $\mathrm{CH}_{2} \mathrm{Cl}_{2}$. The combined organic layer was washed with $\mathrm{H}_{2} \mathrm{O}$, brine, dried $\left(\mathrm{MgSO}_{4}\right)$ and evaporated. The purification by preparative TLC plates of the residue afforded of the desired product, 18a-c or 19a-c, as indicated below for each case.

( \pm )-3-ethyl-2,5-dioxo-1,3a,4,6,6a-pentahydro-pyrrolo[2,3- $d]$ carbazole-

7-carboxylic acid tert-butyl ester 18a

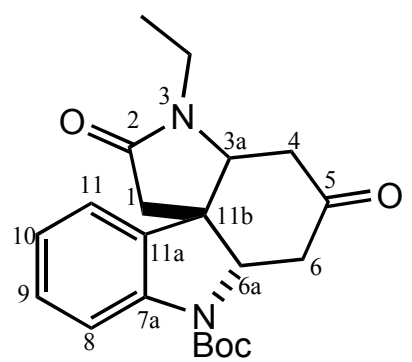

$18 \mathbf{a}$

3-hydro-1H-quinolin-2-one-4,1'-(cyclohex-2',5'-en-4'-one)-carboxylic acid tert-butyl ester 9a (99.7 mg, $0.31 \mathrm{mmol}), \mathrm{EtNH}_{2}(12 \mathrm{~mL})$, dry THF (2 mL), NaH (4.1 mg, 0.10 
mmol) were used to produce 18a. Purification: $\mathrm{CH}_{2} \mathrm{Cl}_{2} / \mathrm{MeOH}$ (9.5:0.5). Yellow amorphous solid (40.1 mg, 35\%).

H.R.M.S. (I.C., m/z) calcd for $\mathrm{C}_{20} \mathrm{H}_{24} \mathrm{~N}_{2} \mathrm{NaO}_{4}{ }^{+}\left(\mathrm{MNa}^{+}\right)$393.1790, found : 393.1811 .

I.R. $\left(\mathrm{CHCl}_{3}\right) \vee\left(\mathrm{cm}^{-1}\right): 1691(\mathrm{C}=\mathrm{O}), 1600(\mathrm{C}=\mathrm{C}), 1481$ (C-Car).

M. S. (E.S.I., $\mathrm{m} / \mathrm{z}): 393\left(\mathrm{MNa}^{+}\right)$.

${ }^{1} \mathbf{H ~ R M N ~}\left(\mathrm{CDCl}_{3}, 300 \mathrm{MHz}\right) \delta(\mathrm{ppm}): 7.67\left(1 \mathrm{H}, \mathrm{d}, \mathrm{J}=7.4 \mathrm{~Hz}, \mathrm{H}_{8}\right), 7.23(1 \mathrm{H}, \mathrm{t}, \mathrm{J}=7.4$ $\left.\mathrm{Hz}, \mathrm{H}_{9}\right), 7.15\left(1 \mathrm{H}, \mathrm{d}, \mathrm{J}=8.9 \mathrm{~Hz}, \mathrm{H}_{11}\right), 6.99\left(1 \mathrm{H}, \mathrm{t}, \mathrm{J}=7.4 \mathrm{~Hz}, \mathrm{H}_{10}\right), 4.41(1 \mathrm{H}, \mathrm{dd}, \mathrm{J}=$ $\left.4.6 \mathrm{~Hz}, \mathrm{~J}=8.1 \mathrm{~Hz}, \mathrm{H}_{6 \mathrm{a}}\right), 4.01\left(1 \mathrm{H}, \mathrm{dd}, \mathrm{J}=7.8 \mathrm{~Hz}, \mathrm{~J}=3.8 \mathrm{~Hz}, \mathrm{H}_{3 \mathrm{a}}\right), 3.69(1 \mathrm{H}, \mathrm{dq}, \mathrm{J}=7.2$ $\mathrm{Hz}, \mathrm{J}=14.5 \mathrm{~Hz}, \mathrm{H}_{1}$ ) $), 2.98\left(1 \mathrm{H}, \mathrm{dq}, \mathrm{J}=7.2 \mathrm{~Hz}, \mathrm{~J}=14.0 \mathrm{~Hz}, \mathrm{H}_{1}\right.$ ) $), 2.78(1 \mathrm{H}, \mathrm{dd}, \mathrm{J}=4.4$ $\left.\mathrm{Hz}, \mathrm{J}=17.3 \mathrm{~Hz}, \mathrm{H}_{6}\right), 2.76-2.65\left(3 \mathrm{H}, \mathrm{m}, \mathrm{H}_{1}\right.$ and $\left.\mathrm{H}_{6}\right), 2.60(1 \mathrm{H}, \mathrm{dd}, \mathrm{J}=3.8 \mathrm{~Hz}, \mathrm{~J}=17.2$ $\left.\mathrm{Hz}, \mathrm{H}_{4}\right), 2.38\left(1 \mathrm{H}, \mathrm{dd}, \mathrm{J}=8.1 \mathrm{~Hz}, \mathrm{~J}=17.2 \mathrm{~Hz}, \mathrm{H}_{4}\right), 1.50(9 \mathrm{H}, \mathrm{s}, \mathrm{Boc}), 1.13(3 \mathrm{H}, \mathrm{t}, \mathrm{J}=$ $7.2 \mathrm{~Hz}, \mathrm{H}_{2}$, ).

${ }^{13} \mathbf{C ~ R M N}\left(\mathrm{CDCl}_{3}, 75 \mathrm{MHz}\right) \delta(\mathrm{ppm}): 204.3\left(\mathrm{C}_{5}\right), 170.7\left(\mathrm{C}_{2}\right), 150.8$ (CO (Boc)), 140.0 $\left(\mathrm{C}_{7 \mathrm{a}}\right), 132.7\left(\mathrm{C}_{11 \mathrm{a}}\right), 128.6\left(\mathrm{C}_{9}\right), 122.7\left(\mathrm{C}_{10}\right), 121.1\left(\mathrm{C}_{11}\right), 114.8\left(\mathrm{C}_{8}\right), 81.5(\mathrm{C}(\mathrm{Boc})), 63.8$ $\left(\mathrm{C}_{6 \mathrm{a}}\right), 60.1\left(\mathrm{C}_{3 \mathrm{a}}\right), 52.4\left(\mathrm{C}_{11 \mathrm{~b}}\right), 44.2\left(\mathrm{C}_{6}\right), 40.1\left(\mathrm{C}_{1}\right), 39.1\left(\mathrm{C}_{4}\right), 34.4\left(\mathrm{C}_{1}\right), 27.4$ $\left(\mathrm{CH}_{3}(\mathrm{Boc})\right), 11.9\left(\mathrm{C}_{2},\right)$.

(土)-9-methoxy-3-ethyl-2,5-dioxo-1,3a,4,6,6a-pentahydro-pyrrolo[2,3- $d]$ carbazole7-carboxylic acid tert-butyl ester $\mathbf{1 8 b}$

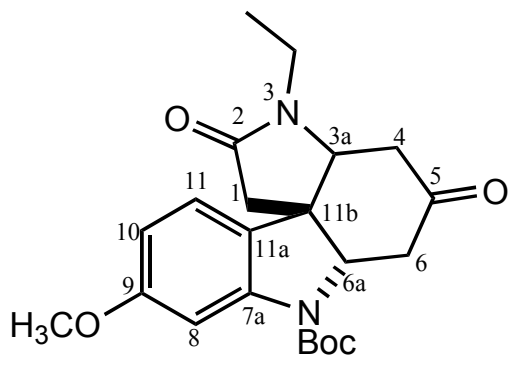

$18 b$

7-methoxy-3-hydro-1H-quinolin-2-one-4,1'-(cyclohex-2',5'-en-4'-one)-carboxylic acid tert-butyl ester 9b (92.4 mg, $0.26 \mathrm{mmol}), \mathrm{EtNH}_{2}(10 \mathrm{~mL})$, dry THF (2 mL), NaH (6.7 $\mathrm{mg}, 0.17 \mathrm{mmol}$ ) were used to produce 18b. Purification: $\mathrm{CH}_{2} \mathrm{Cl}_{2} / \mathrm{MeOH}(9.5: 0.5)$. Yellow amorphous solid (27.8 mg, 27\%).

H.R.M.S. (I.C., m/z) calcd for $\mathrm{C}_{22} \mathrm{H}_{28} \mathrm{~N}_{2} \mathrm{NaO}_{5}{ }^{+}\left(\mathrm{MNa}^{+}\right)$423.1896, found : 423.1907 .

I.R. $\left(\mathrm{CHCl}_{3}\right) \vee\left(\mathrm{cm}^{-1}\right)$ : $1687(\mathrm{C}=\mathrm{O}), 1610(\mathrm{C}=\mathrm{C}), 1497$ (C-Car), 1255 (Car-O).

M. S. (E.S.I., m/z) : $423\left(\mathrm{MNa}^{+}\right)$. 
1H RMN $\left(\mathrm{CDCl}_{3}, 300 \mathrm{MHz}\right) \delta(\mathrm{ppm}): 7.38\left(1 \mathrm{H}, \mathrm{sl}, \mathrm{H}_{8}\right), 7.10\left(1 \mathrm{H}, \mathrm{d}, \mathrm{J}=8.4 \mathrm{~Hz}, \mathrm{H}_{11}\right)$, $6.59\left(1 \mathrm{H}, \mathrm{dd}, \mathrm{J}=8.3 \mathrm{~Hz}, \mathrm{~J}=2.4 \mathrm{~Hz}, \mathrm{H}_{10}\right), 4.47\left(1 \mathrm{H}, \mathrm{dd}, \mathrm{J}=4.8 \mathrm{~Hz}, \mathrm{~J}=7.7 \mathrm{~Hz}, \mathrm{H}_{6 \mathrm{a}}\right)$, $4.03\left(1 \mathrm{H}, \mathrm{dd}, \mathrm{J}=7.8 \mathrm{~Hz}, \mathrm{~J}=3.8 \mathrm{~Hz}, \mathrm{H}_{3 \mathrm{a}}\right), 3.81\left(3 \mathrm{H}, \mathrm{s}, \mathrm{OCH}_{3}\right), 3.74(1 \mathrm{H}, \mathrm{dq}, \mathrm{J}=7.3 \mathrm{~Hz}$, $\left.\mathrm{J}=14.6 \mathrm{~Hz}, \mathrm{H}_{1}{ }^{\prime}\right), 3.03\left(1 \mathrm{H}, \mathrm{dq}, \mathrm{J}=7.1 \mathrm{~Hz}, \mathrm{~J}=14.1 \mathrm{~Hz}, \mathrm{H}_{1}{ }^{\prime}\right), 2.83(1 \mathrm{H}, \mathrm{dd}, \mathrm{J}=4.8 \mathrm{~Hz}, \mathrm{~J}$ $\left.=17.4 \mathrm{~Hz}, \mathrm{H}_{6}\right), 2.76\left(3 \mathrm{H}, \mathrm{m}, \mathrm{H}_{1}\right.$ and $\left.\mathrm{H}_{6}\right), 2.67\left(1 \mathrm{H}, \mathrm{dd}, \mathrm{J}=3.8 \mathrm{~Hz}, \mathrm{~J}=17.2 \mathrm{~Hz}, \mathrm{H}_{4}\right)$, $2.45\left(1 \mathrm{H}, \mathrm{dd}, \mathrm{J}=7.9 \mathrm{~Hz}, \mathrm{~J}=17.2 \mathrm{~Hz}, \mathrm{H}_{4}\right), 1.58(9 \mathrm{H}, \mathrm{s}, \mathrm{Boc}), 1.19$ (3H, dq, J = 7.3 Hz, $\mathrm{H}_{2}$ ).

${ }^{13} \mathbf{C}$ RMN $\left(\mathrm{CDCl}_{3}, 75 \mathrm{MHz}\right) \delta(\mathrm{ppm}): 205.5\left(\mathrm{C}_{5}\right), 171.9\left(\mathrm{C}_{2}\right), 161.0\left(\mathrm{C}_{9}\right), 151.7(\mathrm{CO}$ (Boc)), $142.4\left(\mathrm{C}_{7 \mathrm{a}}\right), 125.5\left(\mathrm{C}_{11 \mathrm{a}}\right), 122.6\left(\mathrm{C}_{11}\right), 109.9\left(\mathrm{C}_{10}\right), 101.7\left(\mathrm{C}_{8}\right), 82.5(\mathrm{C}(\mathrm{Boc}))$, $65.5\left(\mathrm{C}_{6 \mathrm{a}}\right), 61.4\left(\mathrm{C}_{3 \mathrm{a}}\right), 55.6\left(\mathrm{OCH}_{3}\right), 46.3\left(\mathrm{C}_{11 \mathrm{~b}}\right), 45.3\left(\mathrm{C}_{6}\right), 41.2\left(\mathrm{C}_{1}\right), 40.0\left(\mathrm{C}_{4}\right), 35.4$ $\left(\mathrm{C}_{1},\right), 28.4\left(\mathrm{CH}_{3}(\mathrm{Boc})\right), 12.9\left(\mathrm{C}_{2},\right)$.

(士)-8-methoxy-3-ethyl-2,5-dioxo-1,3a,4,6,6a-pentahydro-pyrrolo[2,3- $d]$ carbazole7-carboxylic acid tert-butyl ester 18c

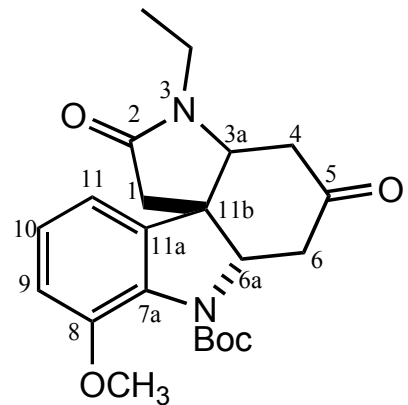

$18 c$

8-methoxy-3-hydro-1H-quinolin-2-one-4,1'-(cyclohex-2',5'-en-4'-one)-carboxylic acid tert-butyl ester 9c $(87.6 \mathrm{mg}, 0.25 \mathrm{mmol}), \mathrm{EtNH}_{2}(10 \mathrm{~mL})$, dry THF $(2 \mathrm{~mL}), \mathrm{NaH}(4.3$ $\mathrm{mg}, 0.11 \mathrm{mmol})$ were used to produce 18c. Purification : $\mathrm{CH}_{2} \mathrm{Cl}_{2} / \mathrm{MeOH}(9.5: 0.5)$. Yellow amorphous solid (62.7 mg, 63\%).

H.R.M.S. (I.C., m/z) calcd for $\mathrm{C}_{22} \mathrm{H}_{28} \mathrm{~N}_{2} \mathrm{NaO}_{5}{ }^{+}\left(\mathrm{MNa}^{+}\right)$423.1890, found : 423.1879 .

I.R. $\left(\mathrm{CHCl}_{3}\right) \vee\left(\mathrm{cm}^{-1}\right)$ : $1706(\mathrm{C}=\mathrm{O}(\mathrm{Boc})), 1676(\mathrm{C}=\mathrm{O}$ (lactame)), 1488 (C-Car), 1255 (Car-O), 1158 (C-O (Boc)).

M. S. (E.S.I., $\mathrm{m} / \mathrm{z}): 423\left(\mathrm{MNa}^{+}\right)$.

1H RMN $\left(\mathrm{CDCl}_{3}, 300 \mathrm{MHz}\right) \delta(\mathrm{ppm}): 7.12\left(1 \mathrm{H}, \mathrm{t}, \mathrm{J}=8.2 \mathrm{~Hz}, \mathrm{~J}=7.6 \mathrm{~Hz}, \mathrm{H}_{10}\right), 6.89$ $\left(1 \mathrm{H}, \mathrm{d}, \mathrm{J}=8.2 \mathrm{~Hz}, \mathrm{H}_{9}\right), 6.81\left(1 \mathrm{H}, \mathrm{d}, \mathrm{J}=7.3 \mathrm{~Hz}, \mathrm{H}_{11}\right), 4.69(1 \mathrm{H}, \mathrm{dd}, \mathrm{J}=4.9 \mathrm{~Hz}, \mathrm{~J}=11.6$ $\left.\mathrm{Hz}, \mathrm{H}_{6 \mathrm{a}}\right), 4.11\left(1 \mathrm{H}, \mathrm{dd}, \mathrm{J}=10.1 \mathrm{~Hz}, \mathrm{~J}=4.0 \mathrm{~Hz}, \mathrm{H}_{3 \mathrm{a}}\right), 3.87\left(3 \mathrm{H}, \mathrm{s}, \mathrm{OCH}_{3}\right), 3.82(1 \mathrm{H}, \mathrm{m}$, $\left.\mathrm{H}_{1}\right), 3.06\left(1 \mathrm{H}, \mathrm{m}, \mathrm{H}_{1}\right), 2.86\left(1 \mathrm{H}, \mathrm{dd}, \mathrm{J}=4.9 \mathrm{~Hz}, \mathrm{~J}=16.8 \mathrm{~Hz}, \mathrm{H}_{6}\right), 2.87-2.62(2 \mathrm{H}$, system $\left.\mathrm{AB}, \mathrm{J}=16.8 \mathrm{~Hz}, \mathrm{H}_{1}\right), 2.80\left(1 \mathrm{H}, \mathrm{dd}, \mathrm{J}=4.0 \mathrm{~Hz}, \mathrm{~J}=16.2 \mathrm{~Hz}, \mathrm{H}_{4}\right), 2.41(1 \mathrm{H}, \mathrm{dd}, \mathrm{J}$ 
$\left.=11.9 \mathrm{~Hz}, \mathrm{~J}=16.8 \mathrm{~Hz}, \mathrm{H}_{6}\right), 2.38\left(1 \mathrm{H}, \mathrm{dd}, \mathrm{J}=9.8 \mathrm{~Hz}, \mathrm{~J}=16.5 \mathrm{~Hz}, \mathrm{H}_{4}\right), 1.50(9 \mathrm{H}, \mathrm{s}$, Boc), $1.24\left(3 \mathrm{H}, \mathrm{t}, \mathrm{J}=7.3 \mathrm{~Hz}, \mathrm{H}_{2}\right.$ ) .

${ }^{13} \mathbf{C ~ R M N}\left(\mathrm{CDCl}_{3}, 75 \mathrm{MHz}\right) \delta(\mathrm{ppm}): 205.3\left(\mathrm{C}_{5}\right), 171.2\left(\mathrm{C}_{2}\right), 153.0(\mathrm{CO}(\mathrm{Boc})), 150.5$ $\left(\mathrm{C}_{8}\right), 139.2\left(\mathrm{C}_{11 \mathrm{a}}\right), 128.8\left(\mathrm{C}_{7 \mathrm{a}}\right), 126.9\left(\mathrm{C}_{10}\right), 113.6\left(\mathrm{C}_{11}\right), 112.8\left(\mathrm{C}_{9}\right), 81.5(\mathrm{C}(\mathrm{Boc})), 67.0$ $\left(\mathrm{C}_{6 \mathrm{a}}\right), 59.3\left(\mathrm{C}_{3 \mathrm{a}}\right), 55.6\left(\mathrm{OCH}_{3}\right), 48.3\left(\mathrm{C}_{11 \mathrm{~b}}\right), 44.1\left(\mathrm{C}_{1}\right), 42.8\left(\mathrm{C}_{6}\right), 41.4\left(\mathrm{C}_{4}\right), 35.5\left(\mathrm{C}_{1}\right)$, $28.1\left(\mathrm{CH}_{3}(\mathrm{Boc})\right), 12.9\left(\mathrm{C}_{2},\right)$.

( \pm )-3-butyl-2,5-dioxo-1,3a,4,6,6a-pentahydro-pyrrolo[2,3- $d$ ]carbazole7-carboxylic acid tert-butyl ester 19a

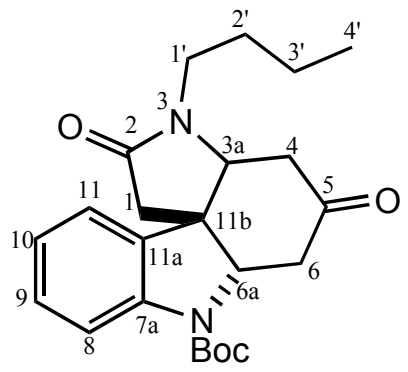

$19 \mathbf{a}$

3-hydro-1H-quinolin-2-one-4,1'-(cyclohex-2',5'-en-4'-one)-carboxylic acid tert-butyl ester 9a (105.8 mg, $0.32 \mathrm{mmol}), \mathrm{BuNH}_{2}(10 \mathrm{~mL})$, dry THF (2 mL), NaH (5.8 mg, 0.14 mmol) were used to produce 19a. Purification: $\mathrm{CH}_{2} \mathrm{Cl}_{2} / \mathrm{MeOH}$ (9.5:0.5). Yellow amorphous solid (61.9 $\mathrm{mg}, 48 \%)$.

H.R.M.S. (I.C., m/z) calcd for $\mathrm{C}_{23} \mathrm{H}_{30} \mathrm{~N}_{2} \mathrm{NaO}_{5}{ }^{+}\left(\mathrm{MNa}^{+}\right)$421.2103, found : 421.2103 .

I.R. $\left(\mathrm{CHCl}_{3}\right) \vee\left(\mathrm{cm}^{-1}\right): 1688(\mathrm{C}=\mathrm{O}), 1480(\mathrm{C}$-Car$)$.

M. S. (E.S.I., m/z) : $421\left(\mathrm{MNa}^{+}\right), 399(\mathrm{M}+\mathrm{H})^{+}$.

${ }^{1} \mathbf{H}$ RMN $\left(\mathrm{CDCl}_{3}, 300 \mathrm{MHz}\right) \delta(\mathrm{ppm}): 7.74\left(1 \mathrm{H}, \mathrm{d}, \mathrm{J}=7.7 \mathrm{~Hz}, \mathrm{H}_{8}\right), 7.30(1 \mathrm{H}, \mathrm{t}, \mathrm{J}=7.7$ $\left.\mathrm{Hz}, \mathrm{H}_{9}\right), 7.23\left(1 \mathrm{H}, \mathrm{d}, \mathrm{J}=7.4 \mathrm{~Hz}, \mathrm{H}_{11}\right), 7.07\left(1 \mathrm{H}, \mathrm{t}, \mathrm{J}=7.4 \mathrm{~Hz}, \mathrm{H}_{10}\right), 4.47(1 \mathrm{H}, \mathrm{dd}, \mathrm{J}=7.8$ $\left.\mathrm{Hz}, \mathrm{J}=4.8 \mathrm{~Hz}, \mathrm{H}_{6 \mathrm{a}}\right), 4.06\left(1 \mathrm{H}, \mathrm{dd}, \mathrm{J}=7.7 \mathrm{~Hz}, \mathrm{~J}=3.8 \mathrm{~Hz}, \mathrm{H}_{3 \mathrm{a}}\right), 3.75-3.64\left(1 \mathrm{H}, \mathrm{m}, \mathrm{H}_{1}{ }^{\prime}\right)$, 3.00-2.90 (1H, m, $\mathrm{H}_{1}$ ) $)$, 2.88-2.72 $\left(4 \mathrm{H}, \mathrm{m}, \mathrm{H}_{1}\right.$ and $\left.\mathrm{H}_{4}\right), 2.67(1 \mathrm{H}, \mathrm{dd}, \mathrm{J}=17.2 \mathrm{~Hz}, \mathrm{~J}=3.8$ $\left.\mathrm{Hz}, \mathrm{H}_{6}\right), 2.47\left(1 \mathrm{H}, \mathrm{dd}, \mathrm{J}=17.2 \mathrm{~Hz}, \mathrm{~J}=7.8 \mathrm{~Hz}, \mathrm{H}_{6}\right), 1.57(9 \mathrm{H}, \mathrm{s}, \mathrm{Boc}), 1.38(2 \mathrm{H}, \mathrm{sx}, \mathrm{J}=$ $\left.7.4 \mathrm{~Hz}, \mathrm{H}_{3}{ }^{\prime}\right), 1.26\left(2 \mathrm{H}, \mathrm{sl}, \mathrm{H}_{2}{ }^{\prime}\right), 0.97\left(3 \mathrm{H}, \mathrm{t}, \mathrm{J}=7.4 \mathrm{~Hz}, \mathrm{H}_{4}{ }^{\prime}\right)$.

${ }^{13} \mathbf{C ~ R M N}\left(\mathrm{CDCl}_{3}, 75 \mathrm{MHz}\right) \delta(\mathrm{ppm}): 205.4\left(\mathrm{C}_{5}\right), 171.9\left(\mathrm{C}_{2}\right), 151.8$ (CO (Boc)), 141.1 $\left(\mathrm{C}_{7 \mathrm{a}}\right), 135.6\left(\mathrm{C}_{11 \mathrm{a}}\right), 129.6\left(\mathrm{C}_{9}\right), 123.7\left(\mathrm{C}_{10}\right), 122.1\left(\mathrm{C}_{11}\right), 115.8\left(\mathrm{C}_{8}\right), 82.5(\mathrm{C}(\mathrm{Boc})), 64.9$ $\left(\mathrm{C}_{6 \mathrm{a}}\right), 61.6\left(\mathrm{C}_{3 \mathrm{a}}\right), 46.8\left(\mathrm{C}_{11 \mathrm{~b}}\right), 45.1\left(\mathrm{C}_{1}\right), 41.0\left(\mathrm{C}_{6}\right), 40.7\left(\mathrm{C}_{4}\right), 40.0\left(\mathrm{C}_{1}\right), 29.7\left(\mathrm{C}_{2}\right), 28.5$ $\left(\mathrm{CH}_{3}(\mathrm{Boc})\right), 20.3\left(\mathrm{C}_{3}{ }^{\prime}\right), 13.8\left(\mathrm{C}_{4}{ }^{\prime}\right)$. 
(土)-9-methoxy-3-butyl-2,5-dioxo-1,3a,4,6,6a-pentahydro-pyrrolo[2,3- $d]$ carbazole7-carboxylic acid tert-butyl ester 19b

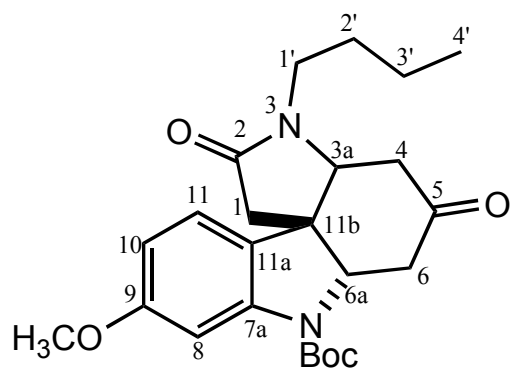

19b

7-methoxy-3-hydro-1H-quinolin-2-one-4,1'-(cyclohex-2',5'-en-4'-one)-carboxylic acid tert-butyl ester $9 \mathbf{b}(89.3 \mathrm{mg}, 0.25 \mathrm{mmol}), \mathrm{BuNH}_{2}(10 \mathrm{~mL})$, dry THF $(2 \mathrm{~mL}), \mathrm{NaH}(2.6$ $\mathrm{mg}, 0.06 \mathrm{mmol}$ ) were used to produce 19b. Purification: $\mathrm{CH}_{2} \mathrm{Cl}_{2} / \mathrm{MeOH}(9.5: 0.5)$. Yellow amorphous solid (32.0 mg, 30\%).

H.R.M.S. (I.C., m/z) calcd for $\mathrm{C}_{24} \mathrm{H}_{32} \mathrm{~N}_{2} \mathrm{NaO}_{5}{ }^{+}\left(\mathrm{MNa}^{+}\right)$451.2209, found : 451.2245.

I.R. $\left(\mathrm{CHCl}_{3}\right) \vee\left(\mathrm{cm}^{-1}\right): 1688(\mathrm{C}=\mathrm{O}), 1498$ (C-Car), 1255 (Car-O).

M. S. (E.S.I., $\mathrm{m} / \mathrm{z}): 451\left(\mathrm{MNa}^{+}\right)$.

1H RMN $\left(\mathrm{CDCl}_{3}, 300 \mathrm{MHz}\right) \delta(\mathrm{ppm}): 7.38\left(1 \mathrm{H}, \mathrm{sl}, \mathrm{H}_{8}\right), 7.09\left(1 \mathrm{H}, \mathrm{d}, \mathrm{J}=8.4 \mathrm{~Hz}, \mathrm{H}_{11}\right)$, $6.60\left(1 \mathrm{H}, \mathrm{dd}, \mathrm{J}=8.4 \mathrm{~Hz}, \mathrm{~J}=2.4 \mathrm{~Hz}, \mathrm{H}_{10}\right), 4.46\left(1 \mathrm{H}, \mathrm{t}, \mathrm{J}=6.0 \mathrm{~Hz}, \mathrm{H}_{6 \mathrm{a}}\right), 4.01(1 \mathrm{H}, \mathrm{dd}, \mathrm{J}$ $\left.=7.6 \mathrm{~Hz}, \mathrm{~J}=3.6 \mathrm{~Hz}, \mathrm{H}_{3 \mathrm{a}}\right), 3.81\left(3 \mathrm{H}, \mathrm{s}, \mathrm{OCH}_{3}\right), 3.74-3.63\left(1 \mathrm{H}, \mathrm{m}, \mathrm{H}_{1}\right), 2.98-2.88(1 \mathrm{H}$, m, $\left.\mathrm{H}_{1}\right)$ ), 2.87-2.75 (4H, m, $\mathrm{H}_{1}$ and $\left.\mathrm{H}_{6}\right), 2.65\left(1 \mathrm{H}, \mathrm{dd}, \mathrm{J}=17.3 \mathrm{~Hz}, \mathrm{~J}=3.7 \mathrm{~Hz}, \mathrm{H}_{4}\right), 2.46$ $\left(1 \mathrm{H}, \mathrm{dd}, \mathrm{J}=17.3 \mathrm{~Hz}, \mathrm{~J}=7.6 \mathrm{~Hz}, \mathrm{H}_{3 \mathrm{a}}\right), 1.57(9 \mathrm{H}, \mathrm{s}, \mathrm{Boc}), 1.37$ (2H, q, J = 7.3 Hz, H${ }^{\prime}$ ), $1.26\left(2 \mathrm{H}, \mathrm{sl}, \mathrm{H}_{2}{ }^{\prime}\right), 0.96\left(3 \mathrm{H}, \mathrm{t}, \mathrm{J}=7.3 \mathrm{~Hz}, \mathrm{H}_{4}{ }^{\prime}\right)$.

${ }^{13} \mathbf{C ~ R M N}\left(\mathrm{CDCl}_{3}, 75 \mathrm{MHz}\right) \delta(\mathrm{ppm}): 205.6\left(\mathrm{C}_{5}\right), 172.1\left(\mathrm{C}_{2}\right), 161.0\left(\mathrm{C}_{9}\right), 151.7(\mathrm{CO}$ (Boc)), $142.5\left(\mathrm{C}_{7 \mathrm{a}}\right), 125.3\left(\mathrm{C}_{11 \mathrm{a}}\right), 122.6\left(\mathrm{C}_{11}\right), 109.8\left(\mathrm{C}_{10}\right), 82.6(\mathrm{C}(\mathrm{Boc})), 65.6\left(\mathrm{C}_{6 \mathrm{a}}\right)$, $61.8\left(\mathrm{C}_{3 \mathrm{a}}\right), 55.6\left(\mathrm{OCH}_{3}\right), 46.3\left(\mathrm{C}_{11 \mathrm{~b}}\right), 45.2\left(\mathrm{C}_{6}\right), 41.1\left(\mathrm{C}_{1}\right), 40.5\left(\mathrm{C}_{1}\right), 39.8\left(\mathrm{C}_{4}\right), 29.7$ $\left(\mathrm{C}_{2},\right), 28.4\left(\mathrm{CH}_{3}(\mathrm{Boc})\right), 20.3\left(\mathrm{C}_{3^{\prime}}\right), 13.8\left(\mathrm{C}_{4}\right)$.

(土)-8-methoxy-3-butyl-2,5-dioxo-1,3a,4,6,6a-pentahydro-pyrrolo[2,3- $d]$ carbazole7-carboxylic acid tert-butyl ester 19c 


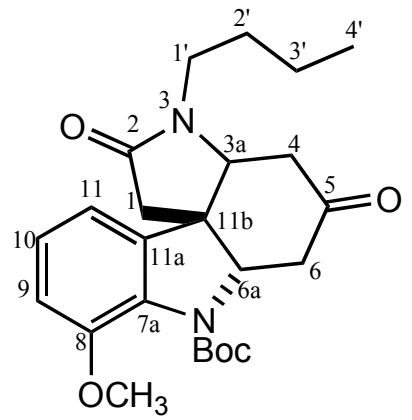

$19 \mathrm{c}$

8-methoxy-3-hydro-1H-quinolin-2-one-4,1'-(cyclohex-2',5'-en-4'-one)-carboxylic acid tert-butyl ester 9c (90.4 mg, $0.25 \mathrm{mmol}), \mathrm{BuNH}_{2}(10 \mathrm{~mL})$, dry THF $(2 \mathrm{~mL}), \mathrm{NaH}(5.3$ $\mathrm{mg}, 0.11 \mathrm{mmol})$ were used to produce 19c. Purification : $\mathrm{CH}_{2} \mathrm{Cl}_{2} / \mathrm{MeOH}(9.5: 0.5)$. Yellow amorphous solid (69.4 mg, 64\%).

H.R.M.S. (I.C., m/z) calcd for $\mathrm{C}_{24} \mathrm{H}_{32} \mathrm{~N}_{2} \mathrm{NaO}_{5}{ }^{+}\left(\mathrm{MNa}^{+}\right)$451.2203, found : 451.3849 .

I.R. $\left(\mathrm{CHCl}_{3}\right) \vee\left(\mathrm{cm}^{-1}\right): 1706(\mathrm{C}=\mathrm{O}(\mathrm{Boc})), 1681(\mathrm{C}=\mathrm{O}$ (lactame)), 1490 (C-Car), 1264 (Car-O), 1160 (C-O (Boc)).

M. S. (E.S.I., $\mathrm{m} / \mathrm{z}): 451\left(\mathrm{MNa}^{+}\right)$.

1H RMN $\left(\mathrm{CDCl}_{3}, 300 \mathrm{MHz}\right) \delta(\mathrm{ppm}): 7.13(1 \mathrm{H}, \mathrm{t}, \mathrm{J}=8.2 \mathrm{~Hz}, \mathrm{~J}=7.6 \mathrm{~Hz}, \mathrm{H10}), 6.89$ $(1 \mathrm{H}, \mathrm{d}, \mathrm{J}=8.2 \mathrm{~Hz}, \mathrm{H} 9), 6.78(1 \mathrm{H}, \mathrm{d}, \mathrm{J}=7.6 \mathrm{~Hz}, \mathrm{H} 11), 4.67(1 \mathrm{H}, \mathrm{dd}, \mathrm{J}=4.9 \mathrm{~Hz}, \mathrm{~J}=11.6$ Hz, H6a), 4.09 (1H, dd, J = 10.1 Hz, J = 4.3 Hz, H3a), $3.87\left(3 \mathrm{H}, \mathrm{s}, \mathrm{OCH}_{3}\right), 3.74(1 \mathrm{H}$, m, H1'), 2.97 (1H, m, H1'), 2.85 (1H, dd, J = 5.2 Hz, J = 16.8 Hz, H6), 2.86-2.63 (2H, system $A B, J=17.1 \mathrm{~Hz}, \mathrm{H1}), 2.79(1 \mathrm{H}, \mathrm{dd}, \mathrm{J}=4.0 \mathrm{~Hz}, \mathrm{~J}=16.2 \mathrm{~Hz}, \mathrm{H} 4), 2.41(1 \mathrm{H}, \mathrm{dd}$, $\mathrm{J}=11.6 \mathrm{~Hz}, \mathrm{~J}=16.8 \mathrm{~Hz}, \mathrm{H} 6), 2.40(1 \mathrm{H}, \mathrm{dd}, \mathrm{J}=10.4 \mathrm{~Hz}, \mathrm{~J}=16.5 \mathrm{~Hz}, \mathrm{H} 4), 1.57$ (2H, m, H2'), 1.50 (9H, s, Boc), 1.41 (2H, m, H3'), 0.98 (3H, t, J = 7.3 Hz, H4').

${ }^{13} \mathrm{C} \mathrm{RMN}\left(\mathrm{CDCl}_{3}, 75 \mathrm{MHz}\right) \delta(\mathrm{ppm})$ : 205.4 (C5), 171.5 (C2), 153.0 (CO (Boc)), 150.5 (C8), 139.2 (C11a), 128.9 (C7a), 126.9 (C10), 113.6 (C11), 112.8 (C9), 81.5 (C (Boc)), 67.1 (C6a), 59.8 (C3a), $55.6\left(\mathrm{OCH}_{3}\right), 48.4$ (C11b), 44.1 (C1), 42.7 (C6), 41.4 (C4), 40.7 (C1'), 29.7 (C2'), 28.2 ( $\mathrm{CH}_{3}$ (Boc)), 20.4 (C3'), 13.8 (C4').

\section{General Procedure for the Synthesis of Carbazole 20a-c}

To 9a-c dissolved in dry $\mathrm{MeOH}, \mathrm{BnNH}_{2}$ was added. After being stirred at room temperature for 4 hours, a solution of $\mathrm{HCl} 1 \mathrm{M}$ was added and the reaction mixture was extracted with $\mathrm{CH}_{2} \mathrm{Cl}_{2}$. The combined organic layer was washed with a solution of $\mathrm{NaOH}(1 \mathrm{M}), \mathrm{H}_{2} \mathrm{O}$, brine, dried $\left(\mathrm{MgSO}_{4}\right)$ and evaporated. The residue was dissolved in a solution of THF/ $\mathrm{NaOH} 1 \mathrm{M}(1 / 1)$. After being stirred at room temperature for 7 hours, 
the reaction mixture was extracted with $\mathrm{CH}_{2} \mathrm{Cl}_{2}$. The combined organic layer was washed with $\mathrm{H}_{2} \mathrm{O}$, brine, dried $\left(\mathrm{MgSO}_{4}\right)$ and evaporated. Silica gel flash-column chromatography of the residue afforded 20a-c as indicated below for each case.

( \pm )-3-benzyl-2,5-dioxo-1,3a,4,6,6a-pentahydro-pyrrolo[2,3- $d]$ carbazole7-carboxylic acid tert-butyl ester 20a

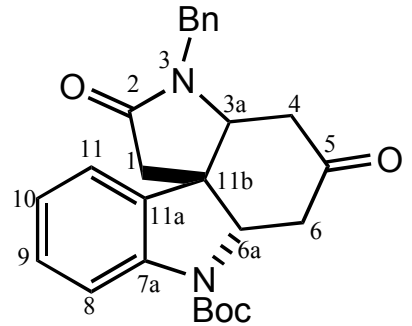

20a

3-hydro-1H-quinolin-2-one-4,1'-(cyclohex-2',5'-en-4'-one)-carboxylic acid tert-butyl ester 9a (500.0 mg, $1.54 \mathrm{mmol})$, dry $\mathrm{MeOH}(8.5 \mathrm{~mL}), \mathrm{BnNH}_{2}(34 \mathrm{~mL})$, THF (10 mL), $\mathrm{NaOH} 1 \mathrm{M}(10 \mathrm{~mL})$ were used to produce 20a. Purification : Heptane/EtOAc (5:5). Pale yellow amorphous solid (523.6 mg, 79\%).

H.R.M.S. (I.C., $\mathrm{m} / \mathrm{z})$ calcd for $\mathrm{C}_{26} \mathrm{H}_{28} \mathrm{~N}_{2} \mathrm{NaO}_{5}{ }^{+}\left(\mathrm{MNa}^{+}\right) 455.1947$, found : 455.1931 .

I.R. $\left(\mathrm{CHCl}_{3}\right) \vee\left(\mathrm{cm}^{-1}\right)$ : $1687(\mathrm{C}=\mathrm{O}), 1480$ (C-Car), 1256 (Car-O).

M. S. (E.S.I., $\mathrm{m} / \mathrm{z}): 455\left(\mathrm{MNa}^{+}\right)$.

1H RMN $\left(\mathrm{CDCl}_{3}, 500 \mathrm{MHz}\right) \delta(\mathrm{ppm}): 7.69\left(1 \mathrm{H}, \mathrm{d}, \mathrm{J}=8.4 \mathrm{~Hz}, \mathrm{H}_{8}\right), 7.41-7.29(5 \mathrm{H}, \mathrm{m}$, Har $(\mathrm{Bn}))$, 7.25-7.20 (1H, m, $\left.\mathrm{H}_{9}\right), 6.95-6.87\left(2 \mathrm{H}, \mathrm{m}, \mathrm{H}_{10}\right.$ and $\left.\mathrm{H}_{11}\right), 5.07(1 \mathrm{H}, \mathrm{d}, \mathrm{J}=14.7$ $\left.\mathrm{Hz}, \mathrm{CH}_{2}(\mathrm{Bn})\right), 4.47\left(1 \mathrm{H}, \mathrm{dd}, \mathrm{J}=8.5 \mathrm{~Hz}, \mathrm{~J}=4.5 \mathrm{~Hz}, \mathrm{H}_{6 \mathrm{a}}\right), 3.97(1 \mathrm{H}, \mathrm{d}, \mathrm{J}=14.7 \mathrm{~Hz}$, $\left.\mathrm{CH}_{2}(\mathrm{Bn})\right), 3.88\left(1 \mathrm{H}, \mathrm{dd}, \mathrm{J}=8.1 \mathrm{~Hz}, \mathrm{~J}=4.0 \mathrm{~Hz}, \mathrm{H}_{3 \mathrm{a}}\right), 2.86-2.79\left(3 \mathrm{H}, \mathrm{m}, \mathrm{H}_{1}\right.$ and $\left.\mathrm{H}_{6}\right)$, 2.66-2.56 (2H, m, $\mathrm{H}_{4}$ and $\left.\mathrm{H}_{6}\right), 2.42\left(1 \mathrm{H}, \mathrm{dd}, \mathrm{J}=8.1 \mathrm{~Hz}, \mathrm{~J}=17.2 \mathrm{~Hz}, \mathrm{H}_{4}\right), 1.56(9 \mathrm{H}, \mathrm{s}$, Boc).

13C RMN $\left(\mathrm{CDCl}_{3}, 75 \mathrm{MHz}\right) \delta(\mathrm{ppm}): 205.3\left(\mathrm{C}_{5}\right), 171.9\left(\mathrm{C}_{2}\right), 151.8$ (CO (Boc)), 140.9 $\left(\mathrm{C}_{7 \mathrm{a}}\right), 135.6$ (Car $\left.(\mathrm{Bn})\right)$, $133.6\left(\mathrm{C}_{11 \mathrm{a}}\right), 129.5$ (Car $\left.(\mathrm{Bn})\right), 129.1$ (Car $\left.(\mathrm{Bn})\right), 128.6$ (Car $(\mathrm{Bn})), 128.3$ (Car $(\mathrm{Bn})$ and $\left.\mathrm{C}_{9}\right), 123.7$ and $122.2\left(\mathrm{C}_{10}\right.$ and $\left.\mathrm{C}_{11}\right), 115.8\left(\mathrm{C}_{8}\right), 82.5(\mathrm{C}$ (Boc)), $64.9\left(\mathrm{C}_{6 \mathrm{a}}\right), 60.8\left(\mathrm{C}_{3 \mathrm{a}}\right), 46.8\left(\mathrm{C}_{11 \mathrm{~b}}\right), 45.2\left(\mathrm{CH}_{2}(\mathrm{Bn})\right), 44.8\left(\mathrm{C}_{1}\right), 41.1\left(\mathrm{C}_{6}\right), 40.0$ $\left(\mathrm{C}_{4}\right), 28.4\left(\mathrm{CH}_{3}(\mathrm{Boc})\right)$.

(士)-9-methoxy-3-benzyl-2,5-dioxo-1,3a,4,6,6a-pentahydro-pyrrolo[2,3- $d]$ carbazole7-carboxylic acid tert-butyl ester 20b 


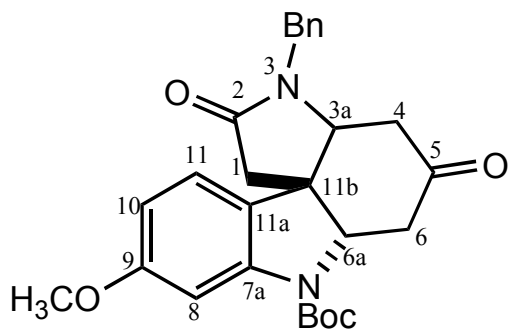

20b

7-methoxy-3-hydro-1H-quinolin-2-one-4,1'-(cyclohex-2',5'-en-4'-one)-carboxylic acid tert-butyl ester 9b (1.0 g, $2.82 \mathrm{mmol})$, dry $\mathrm{MeOH}(15 \mathrm{~mL}), \mathrm{BnNH}_{2}(75 \mathrm{~mL})$, THF (20 $\mathrm{mL}), \quad \mathrm{NaOH} \quad 1 \mathrm{M}(20 \mathrm{~mL})$ were used to produce 20b. Purification: Heptane/EtOAc (5:5). Pale yellow amorphous solid (955.6 mg, 73\%).

H.R.M.S. (I.C., m/z) calcd for $\mathrm{C}_{27} \mathrm{H}_{30} \mathrm{~N}_{2} \mathrm{NaO}_{5}{ }^{+}\left(\mathrm{MNa}^{+}\right)$485.2052, found : 485.2025 .

I.R. $\left(\mathrm{CHCl}_{3}\right) \vee\left(\mathrm{cm}^{-1}\right): 1688(\mathrm{C}=\mathrm{O}), 1610(\mathrm{C}=\mathrm{O}(\mathrm{Boc})), 1496$ (C-Car), 1254 (Car-O).

M. S. (E.S.I., $\mathrm{m} / \mathrm{z}): 485\left(\mathrm{MNa}^{+}\right)$.

1H RMN $\left(\mathrm{CDCl}_{3}, 500 \mathrm{MHz}\right) \delta(\mathrm{ppm}): 7.36-7.26\left(6 \mathrm{H}, \mathrm{m}, \mathrm{H}_{8}\right.$ and $\left.\mathrm{Har}(\mathrm{Bn})\right), 6.78(1 \mathrm{H}$, $\left.\mathrm{d}, \mathrm{J}=8.4 \mathrm{~Hz}, \mathrm{H}_{11}\right), 6.46\left(1 \mathrm{H}, \mathrm{dd}, \mathrm{J}=8.4 \mathrm{~Hz}, \mathrm{~J}=2.4 \mathrm{~Hz}, \mathrm{H}_{10}\right), 5.07(1 \mathrm{H}, \mathrm{d}, \mathrm{J}=14.7 \mathrm{~Hz}$, $\left.\mathrm{CH}_{2}(\mathrm{Bn})\right), 4.47\left(1 \mathrm{H}, \mathrm{dd}, \mathrm{J}=8.2 \mathrm{~Hz}, \mathrm{~J}=4.6 \mathrm{~Hz}, \mathrm{H}_{6 \mathrm{a}}\right), 3.97(1 \mathrm{H}, \mathrm{d}, \mathrm{J}=14.7 \mathrm{~Hz}$, $\left.\mathrm{CH}_{2}(\mathrm{Bn})\right), 3.84\left(1 \mathrm{H}, \mathrm{dd}, \mathrm{J}=7.9 \mathrm{~Hz}, \mathrm{~J}=3.9 \mathrm{~Hz}, \mathrm{H}_{3 \mathrm{a}}\right), 3.77\left(3 \mathrm{H}, \mathrm{s}, \mathrm{OCH}_{3}\right), 2.85-2.77$ $\left(3 \mathrm{H}, \mathrm{m}, \mathrm{H}_{1}\right.$ and $\left.\mathrm{H}_{6}\right), 2.64\left(1 \mathrm{H}, \mathrm{dd}, \mathrm{J}=8.2 \mathrm{~Hz}, \mathrm{~J}=17.2 \mathrm{~Hz}, \mathrm{H}_{6}\right), 2.56(1 \mathrm{H}, \mathrm{dd}, \mathrm{J}=3.9$ $\left.\mathrm{Hz}, \mathrm{J}=17.2 \mathrm{~Hz}, \mathrm{H}_{4}\right), 2.40\left(1 \mathrm{H}, \mathrm{dd}, \mathrm{J}=7.9 \mathrm{~Hz}, \mathrm{~J}=17.2 \mathrm{~Hz}, \mathrm{H}_{4}\right), 1.56$ (9H, s, Boc).

${ }^{13} \mathrm{C}$ RMN $\left(\mathrm{CDCl}_{3}, 75 \mathrm{MHz}\right) \delta(\mathrm{ppm})$ : $205.4\left(\mathrm{C}_{5}\right), 172.1\left(\mathrm{C}_{2}\right), 161.0\left(\mathrm{C}_{9}\right), 151.7(\mathrm{CO}$ (Boc)), $142.3\left(\mathrm{C}_{7 \mathrm{a}}\right), 135.6$ (Car (Bn)), 129.1 (Car $\left.(\mathrm{Bn})\right), 128.6$ (Car (Bn)), 128.3 (Car $(\mathrm{Bn})), 125.3\left(\mathrm{C}_{11 \mathrm{a}}\right), 122.7\left(\mathrm{C}_{11}\right), 109.7\left(\mathrm{C}_{10}\right), 101.8\left(\mathrm{C}_{8}\right), 82.6(\mathrm{C}(\mathrm{Boc})), 65.6\left(\mathrm{C}_{6 \mathrm{a}}\right), 61.0$ $\left(\mathrm{C}_{3 \mathrm{a}}\right), 55.6\left(\mathrm{OCH}_{3}\right), 46.3\left(\mathrm{C}_{11 \mathrm{~b}}\right), 45.3\left(\mathrm{C}_{1}\right), 44.7\left(\mathrm{CH}_{2}(\mathrm{Bn})\right), 41.2\left(\mathrm{C}_{6}\right), 39.9\left(\mathrm{C}_{4}\right), 28.4$ $\left(\mathrm{CH}_{3}(\mathrm{Boc})\right)$.

( \pm )-8-methoxy-3-benzyl-2,5-dioxo-1,3a,4,6,6a-pentahydro-pyrrolo[2,3- $d$ ] carbazole7-carboxylic acid tert-butyl ester 20c

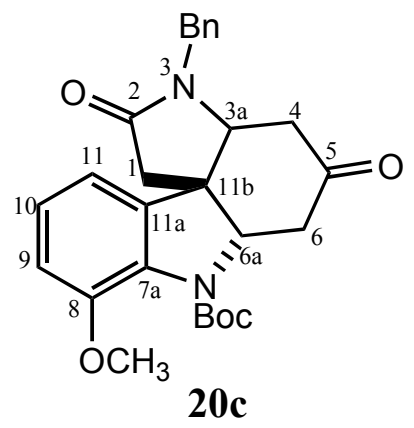


8-methoxy-3-hydro-1H-quinolin-2-one-4,1'-(cyclohex-2',5'-en-4'-one)-carboxylic acid tert-butyl ester 9c (1.0 g, $2.82 \mathrm{mmol}), \mathrm{BnNH}_{2}(55 \mathrm{~mL})$, THF $(20 \mathrm{~mL}), \mathrm{NaOH} 1 \mathrm{M}(20$ $\mathrm{mL})$ were used to produce 20c. Purification: Heptane/EtOAc (5:5). Pale yellow amorphous solid (908.2 $\mathrm{mg}, 70 \%$ ).

H.R.M.S. (I.C., m/z) calcd for $\mathrm{C}_{27} \mathrm{H}_{30} \mathrm{~N}_{2} \mathrm{NaO}_{5}{ }^{+}\left(\mathrm{MNa}^{+}\right)$485.2053, found : 485.2033 .

I.R. $\left(\mathrm{CHCl}_{3}\right) \vee\left(\mathrm{cm}^{-1}\right)$ : $1727(\mathrm{C}=\mathrm{O}(\mathrm{Boc})), 1679(\mathrm{C}=\mathrm{O}$ (lactame)), 1436 (C-Car), 1266 (Car-O), 1160 (C-O (Boc)).

M. S. (E.S.I., $\mathrm{m} / \mathrm{z}): 485\left(\mathrm{MNa}^{+}\right)$.

${ }^{1} \mathbf{H}$ RMN $\left(\mathrm{CDCl}_{3}, 500 \mathrm{MHz}\right) \delta(\mathrm{ppm}): 7.36(5 \mathrm{H}, \mathrm{m}, \operatorname{Har}(\mathrm{Bn})), 6.95(1 \mathrm{H}, \mathrm{t}, \mathrm{J}=7.9 \mathrm{~Hz}$, $\left.\mathrm{H}_{10}\right), 6.81\left(1 \mathrm{H}, \mathrm{d}, \mathrm{J}=8.0 \mathrm{~Hz}, \mathrm{H}_{9}\right), 6.39\left(1 \mathrm{H}, \mathrm{d}, \mathrm{J}=7.3 \mathrm{~Hz}, \mathrm{H}_{11}\right), 5.12(1 \mathrm{H}, \mathrm{d}, \mathrm{J}=14.6$ $\left.\mathrm{Hz}, \mathrm{CH}_{2}(\mathrm{Bn})\right), 4.67\left(1 \mathrm{H}, \mathrm{dd}, \mathrm{J}=5.2 \mathrm{~Hz}, \mathrm{~J}=11.6 \mathrm{~Hz}, \mathrm{H}_{6 \mathrm{a}}\right), 4.00(1 \mathrm{H}, \mathrm{d}, \mathrm{J}=14.6 \mathrm{~Hz}$, $\left.\mathrm{CH}_{2}(\mathrm{Bn})\right), 3.88\left(1 \mathrm{H}, \mathrm{dd}, \mathrm{J}=9.8 \mathrm{~Hz}, \mathrm{~J}=4.0 \mathrm{~Hz}, \mathrm{H}_{3 \mathrm{a}}\right), 3.83\left(3 \mathrm{H}, \mathrm{s}, \mathrm{OCH}_{3}\right), 2.91-2.68$ $\left(2 \mathrm{H}\right.$, system $\left.\mathrm{AB}, \mathrm{J}=16.8 \mathrm{~Hz}, \mathrm{H}_{1}\right), 2.80\left(1 \mathrm{H}, \mathrm{dd}, \mathrm{J}=4.9 \mathrm{~Hz}, \mathrm{~J}=16.8 \mathrm{~Hz}, \mathrm{H}_{6}\right), 2.70(1 \mathrm{H}$, dd, J = 4.0 Hz, J = 16.2 Hz, H $), 2.35\left(1 \mathrm{H}, \mathrm{dd}, \mathrm{J}=10.1 \mathrm{~Hz}, \mathrm{~J}=16.5 \mathrm{~Hz}, \mathrm{H}_{4}\right), 2.30(1 \mathrm{H}$, $\left.\mathrm{dd}, \mathrm{J}=11.6 \mathrm{~Hz}, \mathrm{~J}=16.8 \mathrm{~Hz}, \mathrm{H}_{6}\right), 1.49$ (9H, s, Boc).

${ }^{13} \mathbf{C ~ R M N ~}\left(\mathrm{CDCl}_{3}, 75 \mathrm{MHz}\right) \delta(\mathrm{ppm}): 205.3\left(\mathrm{C}_{5}\right), 171.2\left(\mathrm{C}_{2}\right), 153.0$ (CO (Boc)), 150.2 $\left(\mathrm{C}_{8}\right), 138.5\left(\mathrm{C}_{11 \mathrm{a}}\right), 135.1\left(\mathrm{C}_{7 \mathrm{a}}\right), 128.7$ (Car $\left.(\mathrm{Bn})\right), 128.5$ (Car $\left.(\mathrm{Bn})\right)$, 128.4 (Car $\left.(\mathrm{Bn})\right)$, 128.1 (Car (Bn)), $126.5\left(\mathrm{C}_{10}\right), 113.0\left(\mathrm{C}_{11}\right), 112.4\left(\mathrm{C}_{9}\right), 81.3(\mathrm{C}(\mathrm{Boc})), 67.1\left(\mathrm{C}_{6 \mathrm{a}}\right), 58.5$ $\left(\mathrm{C}_{3 \mathrm{a}}\right), 55.4\left(\mathrm{OCH}_{3}\right), 48.0\left(\mathrm{C}_{11 \mathrm{~b}}\right), 44.6\left(\mathrm{CH}_{2}(\mathrm{Bn})\right), 43.7\left(\mathrm{C}_{1}\right), 42.2\left(\mathrm{C}_{6}\right), 40.9\left(\mathrm{C}_{4}\right), 27.9$ $\left(\mathrm{CH}_{3}(\mathrm{Boc})\right)$.

\section{General Procedure for the Synthesis of Carbazole 21a-c}

To 20a-c dissolved in $\mathrm{CH}_{2} \mathrm{Cl}_{2}$, TFA was added. After being stirred at room temperature for 5 hours, $\mathrm{NaOH} 1 \mathrm{M}$ was added and the reaction mixture was extracted with $\mathrm{CH}_{2} \mathrm{Cl}_{2}$. The combined organic layer was washed with $\mathrm{H}_{2} \mathrm{O}$, brine, dried $\left(\mathrm{MgSO}_{4}\right)$ and evaporated. To the residue dissolved in toluene, $\mathrm{Na}_{2} \mathrm{SO}_{4}$, ethylene glycol and paratoluenesulfonic acid were added successively. After being stirred at $140^{\circ} \mathrm{C}$ for 4 hours, $\mathrm{NaOH} 1 \mathrm{M}$ was added and the reaction mixture was extracted with $\mathrm{CH}_{2} \mathrm{Cl}_{2}$. The combined organic layer was washed with $\mathrm{H}_{2} \mathrm{O}$, brine, dried $\left(\mathrm{MgSO}_{4}\right)$ and evaporated. Silica gel flash-column chromatography of the residue afforded 21a-c as indicated below for each case.

( \pm )-3-benzyl-2-oxo-5-(1',4'-dioxaspiro[4.5])-1,3a,4,6,6a,7hexahydro-pyrrolo[2,3- $d]$ carbazole 21a 


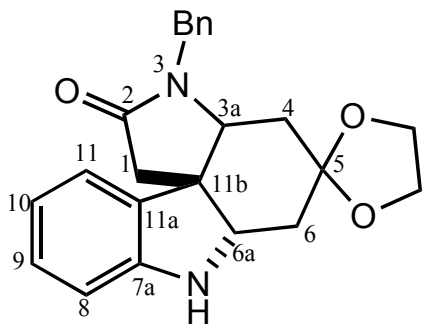

21a

( \pm )-3-benzyl-2,5-dioxo-1,3a,4,6,6a-pentahydro-pyrrolo[2,3- $d$ ]carbazole-7-carboxylic acid tert-butyl ester $\mathbf{2 0 a}(350.3 \mathrm{mg}, 0.81 \mathrm{mmol})$, dry $\mathrm{CH}_{2} \mathrm{Cl}_{2}(20 \mathrm{~mL})$, TFA (2 mL), toluene $(15 \mathrm{~mL}), \mathrm{Na}_{2} \mathrm{SO}_{4}(5 \mathrm{~g})$, ethylene glycol $(226.7 \mu \mathrm{L}, 4.05 \mathrm{mmol})$, and paratoluenesulfonic acid (15.4 mg, $0.08 \mathrm{mmol}$ ) were used to produce 21a. Purification : $\mathrm{CH}_{2} \mathrm{Cl}_{2} / \mathrm{MeOH}$ (9.9:0.1). White amorphous solid (231.3 mg, 76\%).

H.R.M.S. (I.C., m/z) calcd for $\mathrm{C}_{23} \mathrm{H}_{24} \mathrm{~N}_{2} \mathrm{NaO}_{3}{ }^{+}\left(\mathrm{MNa}^{+}\right)$399.1685, found : 399.1671.

I.R. $\left(\mathrm{CHCl}_{3}\right) \vee\left(\mathrm{cm}^{-1}\right)$ : $3333(\mathrm{~N}-\mathrm{H}), 1673(\mathrm{C}=\mathrm{O}), 1482$ (C-Car), 1257 (Car-O), 1049 (C-O).

M. S. (E.S.I., $\mathrm{m} / \mathrm{z}): 399\left(\mathrm{MNa}^{+}\right)$.

1H RMN $\left(\mathrm{CDCl}_{3}, 300 \mathrm{MHz}\right) \delta(\mathrm{ppm}): 7.31-7.25(5 \mathrm{H}, \mathrm{m}, \mathrm{Har}(\mathrm{Bn})), 7.05(1 \mathrm{H}, \mathrm{dd}, \mathrm{J}=$ $\left.7.7 \mathrm{~Hz}, \mathrm{~J}=1.2 \mathrm{~Hz}, \mathrm{H}_{9}\right), 6.93\left(1 \mathrm{H}, \mathrm{d}, \mathrm{J}=7.4 \mathrm{~Hz}, \mathrm{H}_{11}\right), 6.72-6.65\left(2 \mathrm{H}, \mathrm{m}, \mathrm{H}_{8}\right.$ and $\left.\mathrm{H}_{10}\right)$, $5.07\left(1 \mathrm{H}, \mathrm{d}, \mathrm{J}=15.0 \mathrm{~Hz}, \mathrm{CH}_{2}(\mathrm{Bn})\right), 4.07\left(1 \mathrm{H}, \mathrm{d}, \mathrm{J}=15.0 \mathrm{~Hz}, \mathrm{CH}_{2}(\mathrm{Bn})\right), 3.95-3.88$ (5H, $\mathrm{m}$, Hdioxolane and $\left.\mathrm{H}_{6 \mathrm{a}}\right), 3.62\left(1 \mathrm{H}, \mathrm{dd}, \mathrm{J}=7.5 \mathrm{~Hz}, \mathrm{~J}=4.6 \mathrm{~Hz}, \mathrm{H}_{3 \mathrm{a}}\right), 2.71(2 \mathrm{H}$, system $\left.\mathrm{AB}, \mathrm{J}=16.9 \mathrm{~Hz}, \mathrm{H}_{1}\right), 2.02-1.87\left(3 \mathrm{H}, \mathrm{m}, \mathrm{H}_{4}\right.$ and $\left.\mathrm{H}_{6}\right), 1.78(1 \mathrm{H}, \mathrm{dd}, \mathrm{J}=7.5 \mathrm{~Hz}, \mathrm{~J}=14.4$ $\left.\mathrm{Hz}, \mathrm{H}_{4}\right)$.

${ }^{13} \mathbf{C}$ RMN $\left(\mathrm{CDCl}_{3}, 75 \mathrm{MHz}\right) \delta(\mathrm{ppm}): 172.9\left(\mathrm{C}_{2}\right), 149.3\left(\mathrm{C}_{7 \mathrm{a}}\right), 136.3$ (Car (Bn)), 133.4 $\left(\mathrm{C}_{11 \mathrm{a}}\right), 128.8$ (Car (Bn)), 128.6 (Car (Bn)), 128.4 (Car $\left.(\mathrm{Bn})\right), 127.7\left(\mathrm{C}_{9}\right), 122.4\left(\mathrm{C}_{11}\right)$, $119.4\left(\mathrm{C}_{10}\right), 111.1\left(\mathrm{C}_{8}\right), 106.9\left(\mathrm{C}_{5}\right), 64.3$ and 64.4 (Cdioxolane), $63.2\left(\mathrm{C}_{6 \mathrm{a}}\right), 60.5\left(\mathrm{C}_{3 \mathrm{a}}\right)$, $47.4\left(\mathrm{C}_{11 \mathrm{~b}}\right), 44.5\left(\mathrm{CH}_{2}(\mathrm{Bn})\right), 42.3\left(\mathrm{C}_{1}\right), 35.9\left(\mathrm{C}_{6}\right), 34.3\left(\mathrm{C}_{4}\right)$.

( \pm )-9-methoxy-3-benzyl-2-oxo-5-(1',4'-dioxaspiro[4.5])-1,3a,4,6,6a,7hexahydro-pyrrolo[2,3- $d]$ carbazole $\mathbf{2 1 b}$ 


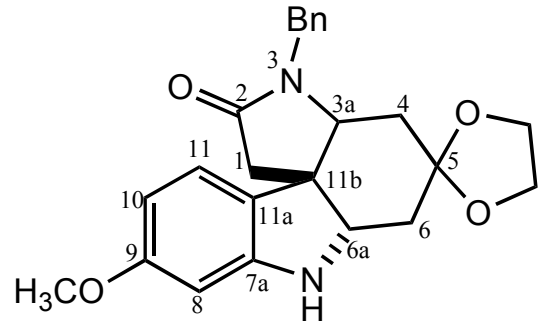

$21 b$

(土)-9-methoxy-3-benzyl-2,5-dioxo-1,3a,4,6,6a-pentahydro-pyrrolo[2,3- $d$ ]carbazole-7carboxylic acid tert-butyl ester $\mathbf{2 0 b}(955.6 \mathrm{mg}, 2.07 \mathrm{mmol})$, dry $\mathrm{CH}_{2} \mathrm{Cl}_{2}(50 \mathrm{~mL}), \mathrm{TFA}$ $(5 \mathrm{~mL})$, toluene $(38 \mathrm{~mL}), \mathrm{Na}_{2} \mathrm{SO}_{4}(18 \mathrm{~g})$, ethylene glycol $(578.3 \mu \mathrm{L}, 10.34 \mathrm{mmol})$, and para-toluenesulfonic acid $(39.3 \mathrm{mg}, 0.21 \mathrm{mmol})$ were used to produce $\mathbf{2 1 b}$. Purification : $\mathrm{CH}_{2} \mathrm{Cl}_{2} / \mathrm{MeOH}$ (9.9:0.1). White amorphous solid (539.7 mg, 64\%).

H.R.M.S. (I.C., m/z) calcd for $\mathrm{C}_{24} \mathrm{H}_{26} \mathrm{~N}_{2} \mathrm{NaO}_{4}{ }^{+}\left(\mathrm{MNa}^{+}\right) 429.1790$, found : 429.1776 .

I.R. $\left(\mathrm{CHCl}_{3}\right) \vee\left(\mathrm{cm}^{-1}\right)$ : $3333(\mathrm{~N}-\mathrm{H}), 1680(\mathrm{C}=\mathrm{O}), 1498$ (C-Car), 1260 (Car-O), 1080 (C-O).

M. S. (E.S.I., $\mathrm{m} / \mathrm{z}): 429\left(\mathrm{MNa}^{+}\right)$.

${ }^{1} \mathbf{H ~ R M N}\left(\mathrm{CDCl}_{3}, 300 \mathrm{MHz}\right) \delta(\mathrm{ppm})$ : 7.28-7.22 (5H, m, Har (Bn)), $6.81(1 \mathrm{H}, \mathrm{d}, \mathrm{J}=$ $\left.7.1 \mathrm{~Hz}, \mathrm{H}_{11}\right), 6.22\left(2 \mathrm{H}, \mathrm{m}, \mathrm{H}_{8}\right.$ and $\left.\mathrm{H}_{10}\right), 5.06\left(1 \mathrm{H}, \mathrm{d}, \mathrm{J}=15.0 \mathrm{~Hz}, \mathrm{CH}_{2}(\mathrm{Bn})\right), 4.06(1 \mathrm{H}, \mathrm{d}$, $\left.\mathrm{J}=15.0 \mathrm{~Hz}, \mathrm{CH}_{2}(\mathrm{Bn})\right), 3.93-3.87\left(5 \mathrm{H}, \mathrm{m}\right.$, Hdioxolane and $\left.\mathrm{H}_{6 \mathrm{a}}\right), 3.72\left(3 \mathrm{H}, \mathrm{s}, \mathrm{OCH}_{3}\right)$, $3.59\left(1 \mathrm{H}, \mathrm{dd}, \mathrm{J}=7.3 \mathrm{~Hz}, \mathrm{~J}=4.6 \mathrm{~Hz}, \mathrm{H}_{3 \mathrm{a}}\right), 2.68\left(2 \mathrm{H}\right.$, system $\left.\mathrm{AB}, \mathrm{J}=17.9 \mathrm{~Hz}, \mathrm{H}_{1}\right), 2.01-$ $1.86\left(3 \mathrm{H}, \mathrm{m}, \mathrm{H}_{4}\right.$ and $\left.\mathrm{H}_{6}\right), 1.77\left(1 \mathrm{H}, \mathrm{dd}, \mathrm{J}=7.4 \mathrm{~Hz}, \mathrm{~J}=14.4 \mathrm{~Hz}, \mathrm{H}_{4}\right)$.

${ }^{13} \mathbf{C ~ R M N}\left(\mathrm{CDCl}_{3}, 75 \mathrm{MHz}\right) \delta(\mathrm{ppm})$ : $172.9\left(\mathrm{C}_{2}\right), 160.7\left(\mathrm{C}_{9}\right), 150.6\left(\mathrm{C}_{7 \mathrm{a}}\right), 136.3(\mathrm{Car}$ (Bn)), 128.7 (Car (Bn)), 128.4 (Car (Bn)), 127.7 (Car (Bn)), $125.7\left(\mathrm{C}_{11 \mathrm{a}}\right), 122.8\left(\mathrm{C}_{11}\right)$, $106.9\left(\mathrm{C}_{5}\right), 104.7\left(\mathrm{C}_{8}\right), 97.5\left(\mathrm{C}_{10}\right), 64.2$ and 64.4 (Cdioxolane), $63.6\left(\mathrm{C}_{6 \mathrm{a}}\right), 60.7\left(\mathrm{C}_{3 \mathrm{a}}\right)$, $55.4\left(\mathrm{OCH}_{3}\right), 46.7\left(\mathrm{C}_{11 \mathrm{~b}}\right), 44.4\left(\mathrm{CH}_{2}(\mathrm{Bn})\right), 42.5\left(\mathrm{C}_{1}\right), 36.0\left(\mathrm{C}_{6}\right), 34.2\left(\mathrm{C}_{4}\right)$.

(士)-8-methoxy-3-benzyl-2-oxo-5-(1',4'-dioxaspiro[4.5])-1,3a,4,6,6a,7hexahydro-pyrrolo[2,3- $d]$ carbazole 21c

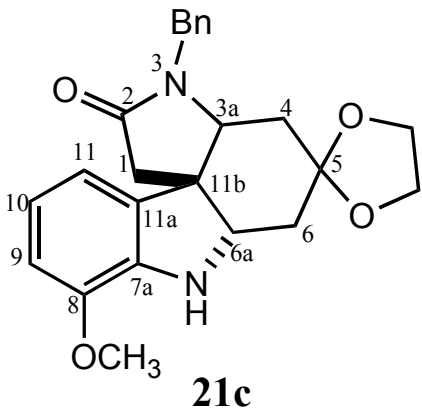


( \pm )-8-methoxy-3-benzyl-2,5-dioxo-1,3a,4,6,6a-pentahydro-pyrrolo[2,3- $d$ ]carbazole-7carboxylic acid tert-butyl ester 20c $(828.4 \mathrm{mg}, 1.79 \mathrm{mmol})$, dry $\mathrm{CH}_{2} \mathrm{Cl}_{2}(45 \mathrm{~mL})$, TFA (4.5 mL), toluene (35 mL), $\mathrm{Na}_{2} \mathrm{SO}_{4}(15 \mathrm{~g})$, ethylene glycol $(501.3 \mu \mathrm{L}, 8.96 \mathrm{mmol})$, and para-toluenesulfonic acid $(34.1 \mathrm{mg}, 0.18 \mathrm{mmol}$ ) were used to produce $21 \mathrm{c}$. Purification : $\mathrm{CH}_{2} \mathrm{Cl}_{2} / \mathrm{MeOH}$ (9.9:0.1). White amorphous solid (491.2 mg, $67 \%$ ).

H.R.M.S. (I.C., $\mathrm{m} / \mathrm{z}$ ) calcd for $\mathrm{C}_{24} \mathrm{H}_{27} \mathrm{~N}_{2} \mathrm{O}_{4}{ }^{+}\left(\mathrm{MH}^{+}\right)$407.1971, found : 407.1960 .

I.R. $\left(\mathrm{CHCl}_{3}\right) \vee\left(\mathrm{cm}^{-1}\right)$ : $3343(\mathrm{~N}-\mathrm{H}), 1680(\mathrm{C}=\mathrm{O}$ (lactame)), 1490 (C-Car), 1252 (CarO), 1013 (C-O).

M. S. (E.S.I., $\mathrm{m} / \mathrm{z}): 429\left(\mathrm{MNa}^{+}\right), 407\left(\mathrm{MH}^{+}\right)$.

$1_{\mathbf{H}} \mathbf{R M N}\left(\mathrm{CDCl}_{3}, 300 \mathrm{MHz}\right) \delta(\mathrm{ppm}): 7.27(5 \mathrm{H}, \mathrm{m}, \mathrm{Har}(\mathrm{Bn})), 6.67\left(2 \mathrm{H}, \mathrm{m}, \mathrm{H}_{9}\right.$ and $\left.\mathrm{H}_{10}\right), 6.57\left(1 \mathrm{H}, \mathrm{dd}, \mathrm{J}=6.4 \mathrm{~Hz}, \mathrm{~J}=2.1 \mathrm{~Hz}, \mathrm{H}_{11}\right), 5.06\left(1 \mathrm{H}, \mathrm{d}, \mathrm{J}=14.9 \mathrm{~Hz}, \mathrm{CH}_{2}(\mathrm{Bn})\right)$, $4.05\left(1 \mathrm{H}, \mathrm{d}, \mathrm{J}=14.9 \mathrm{~Hz}, \mathrm{CH}_{2}(\mathrm{Bn})\right), 3.91\left(5 \mathrm{H}, \mathrm{m}\right.$, Hdioxolane and $\left.\mathrm{H}_{6 \mathrm{a}}\right), 3.79(3 \mathrm{H}, \mathrm{s}$, $\left.\mathrm{OCH}_{3}\right), 3.68\left(1 \mathrm{H}, \mathrm{dd}, \mathrm{J}=4.7 \mathrm{~Hz}, \mathrm{~J}=7.5 \mathrm{~Hz}, \mathrm{H}_{3 \mathrm{a}}\right), 2.81-2.58(2 \mathrm{H}$, system $\mathrm{AB}, \mathrm{J}=16.8$ $\left.\mathrm{Hz}, \mathrm{H}_{1}\right), 1.91\left(3 \mathrm{H}, \mathrm{m}, \mathrm{H}_{4}\right.$ and $\left.\mathrm{H}_{6}\right), 1.78\left(1 \mathrm{H}, \mathrm{dd}, \mathrm{J}=7.5 \mathrm{~Hz}, \mathrm{~J}=14.3 \mathrm{~Hz}, \mathrm{H}_{4}\right)$.

${ }^{13} \mathbf{C ~ R M N}\left(\mathrm{CDCl}_{3}, 75 \mathrm{MHz}\right) \delta(\mathrm{ppm}): 172.8\left(\mathrm{C}_{2}\right), 146.1\left(\mathrm{C}_{8}\right), 138.2\left(\mathrm{C}_{7 \mathrm{a}}\right), 136.2(\mathrm{Car}$ $(\mathrm{Bn})), 134.3\left(\mathrm{C}_{11 \mathrm{a}}\right), 128.7$ (Car (Bn)), $128.4(\mathrm{Car}(\mathrm{Bn})), 127.6(\mathrm{Car}(\mathrm{Bn})), 120.5\left(\mathrm{C}_{10}\right)$, $114.5\left(\mathrm{C}_{11}\right), 110.2\left(\mathrm{C}_{9}\right), 106.9\left(\mathrm{C}_{5}\right), 64.3$ (Cdioxolane), $63.5\left(\mathrm{C}_{6 \mathrm{a}}\right), 60.3\left(\mathrm{C}_{3 \mathrm{a}}\right), 55.2$ $\left(\mathrm{OCH}_{3}\right), 48.2\left(\mathrm{C}_{11 \mathrm{~b}}\right), 44.5\left(\mathrm{CH}_{2}(\mathrm{Bn})\right), 42.3\left(\mathrm{C}_{1}\right), 36.0\left(\mathrm{C}_{6}\right), 34.4\left(\mathrm{C}_{4}\right)$.

\section{General Procedure for the Synthesis of Carbazole 22a-c}

To 21a-c dissolved in dry THF, $\mathrm{LiAlH}_{4} 1 \mathrm{M}$ in THF was added. The reaction mixture was stirred for 5 hours at $60^{\circ} \mathrm{C}$. After addition of potassium sodium tartrate at $0^{\circ} \mathrm{C}$, the reaction mixture was extracted with $\mathrm{CH}_{2} \mathrm{Cl}_{2}$. The combined organic layer was washed with $\mathrm{H}_{2} \mathrm{O}$, brine, dried $\left(\mathrm{MgSO}_{4}\right)$ and evaporated. Alumina gel flash-column chromatography of the residue afforded 22a-c as indicated below for each case.

( \pm )-3-benzyl-5-(1',4'-dioxaspiro[4.5])-1,2,3a,4,6,6a,7heptahydro-pyrrolo[2,3- $d]$ carbazole 22a 


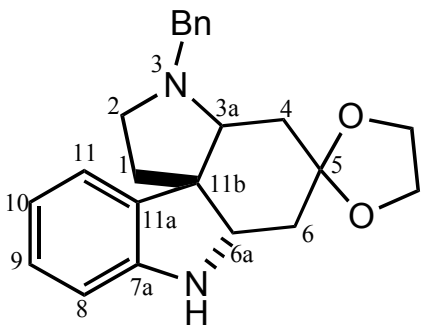

$22 \mathbf{a}$

( \pm )-3-benzyl-2-oxo-5-(1',4'-dioxaspiro[4.5])-1,3a,4,6,6a,7-hexahydro-pyrrolo[2,3-d] carbazole $21 \mathrm{a}(287.3 \mathrm{mg}, 0.76 \mathrm{mmol})$, dry THF $(15 \mathrm{~mL})$, and $\mathrm{LiAlH}_{4} 1 \mathrm{M}$ in THF $(3.8$ $\mathrm{mL}, 3.82 \mathrm{mmol}$ ) were used to produce 22a. Purification : Heptane/EtOAc (7:3). White amorphous solid (216.7 mg, 78\%).

H.R.M.S. (I.C., m/z) calcd for $\mathrm{C}_{23} \mathrm{H}_{27} \mathrm{~N}_{2} \mathrm{O}_{3}{ }^{+}\left(\mathrm{MH}^{+}\right)$363.2073, found : 363.2049 .

I.R. $\left(\mathrm{CHCl}_{3}\right) \vee\left(\mathrm{cm}^{-1}\right): 3357(\mathrm{~N}-\mathrm{H}), 1483$ (C-Car), $1042(\mathrm{C}-\mathrm{O})$.

M. S. (E.S.I., m/z) : $363\left(\mathrm{MH}^{+}\right)$.

1H RMN $\left(\mathrm{CDCl}_{3}, 300 \mathrm{MHz}\right) \delta(\mathrm{ppm}): 7.40(2 \mathrm{H}, \mathrm{d}, \mathrm{J}=7.6 \mathrm{~Hz}, \operatorname{Har}(\mathrm{Bn})), 7.30(2 \mathrm{H}, \mathrm{t}, \mathrm{J}$ $=7.6 \mathrm{~Hz}, \operatorname{Har}(\mathrm{Bn})), 7.23(1 \mathrm{H}, \mathrm{t}, \mathrm{J}=7.6 \mathrm{~Hz}, \operatorname{Har}(\mathrm{Bn})), 7.10\left(1 \mathrm{H}, \mathrm{d}, \mathrm{J}=7.3 \mathrm{~Hz}, \mathrm{H}_{11}\right)$, $7.04\left(1 \mathrm{H}, \mathrm{t}, \mathrm{J}=7.6 \mathrm{~Hz}, \mathrm{H}_{9}\right), 6.77\left(1 \mathrm{H}, \mathrm{t}, \mathrm{J}=7.6 \mathrm{~Hz}, \mathrm{H}_{10}\right), 6.66\left(1 \mathrm{H}, \mathrm{d}, \mathrm{J}=7.6 \mathrm{~Hz}, \mathrm{H}_{8}\right)$, $4.05\left(1 \mathrm{H}, \mathrm{d}, \mathrm{J}=12.8 \mathrm{~Hz}, \mathrm{CH}_{2}(\mathrm{Bn})\right), 4.00-3.87$ (4H, m, Hdioxolane), 3.78 (1H, t, J = 4.3 $\left.\mathrm{Hz}, \mathrm{H}_{6 \mathrm{a}}\right), 3.15\left(1 \mathrm{H}, \mathrm{d}, \mathrm{J}=12.6 \mathrm{~Hz}, \mathrm{CH}_{2}(\mathrm{Bn})\right), 2.92\left(1 \mathrm{H}, \mathrm{dt}, \mathrm{J}=2.1 \mathrm{~Hz}, \mathrm{~J}=8.9 \mathrm{~Hz}, \mathrm{H}_{2}\right)$, $2.56\left(1 \mathrm{H}, \mathrm{dd}, \mathrm{J}=5.2 \mathrm{~Hz}, \mathrm{H}_{3 \mathrm{a}}\right), 2.42\left(1 \mathrm{H}, \mathrm{dd}, \mathrm{J}=14.6 \mathrm{~Hz}, \mathrm{~J}=3.7 \mathrm{~Hz}, \mathrm{H}_{6}\right), 2.39(1 \mathrm{H}, \mathrm{q}, \mathrm{J}$ $\left.=8.9 \mathrm{~Hz}, \mathrm{H}_{2}\right), 2.15\left(1 \mathrm{H}, \mathrm{ddd}, \mathrm{J}=13.4 \mathrm{~Hz}, \mathrm{~J}=8.2 \mathrm{~Hz}, \mathrm{~J}=2.1 \mathrm{~Hz}, \mathrm{H}_{1}\right), 2.02-1.97(2 \mathrm{H}$, $\mathrm{m}, \mathrm{H}_{4}$ and $\left.\mathrm{H}_{6}\right), 1.89-1.84\left(2 \mathrm{H}, \mathrm{m}, \mathrm{H}_{4}\right.$ and $\left.\mathrm{H}_{1}\right)$.

${ }^{13} \mathbf{C ~ R M N}\left(\mathrm{CDCl}_{3}, 75 \mathrm{MHz}\right) \delta(\mathrm{ppm}): 150.1\left(\mathrm{C}_{7 \mathrm{a}}\right), 139.7$ (Car (Bn)), $136.7\left(\mathrm{C}_{11 \mathrm{a}}\right)$, 129.0 (Car (Bn)), 128.2 (Car (Bn)), 126.8 (Car (Bn)), $127.8\left(\mathrm{C}_{9}\right), 123.5\left(\mathrm{C}_{11}\right), 119.3$ $\left(\mathrm{C}_{10}\right), 110.4\left(\mathrm{C}_{8}\right), 108.2\left(\mathrm{C}_{5}\right), 68.5\left(\mathrm{C}_{3 \mathrm{a}}\right), 65.1\left(\mathrm{C}_{6 \mathrm{a}}\right), 64.1$ and 63.8 (Cdioxolane), 56.6 $\left(\mathrm{CH}_{2}(\mathrm{Bn})\right), 53.0\left(\mathrm{C}_{11 \mathrm{~b}}\right), 52.2\left(\mathrm{C}_{2}\right), 37.6\left(\mathrm{C}_{1}\right), 35.3\left(\mathrm{C}_{6}\right), 32.1\left(\mathrm{C}_{4}\right)$.

( \pm )-9-methoxy-3-benzyl-5-(1',4'-dioxaspiro[4.5])-1,2,3a,4,6,6a,7heptahydro-pyrrolo[2,3- $d$ ] carbazole 22b

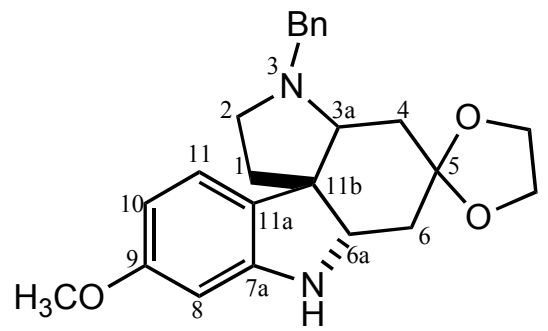

$22 b$ 
( \pm )-9-methoxy-3-benzyl-2-oxo-5-(1',4'-dioxaspiro[4.5])-1,3a,4,6,6a,7-hexahydropyrrolo [2,3- $d$ ] carbazole $21 \mathrm{~b}(97.5 \mathrm{mg}, 0.24 \mathrm{mmol})$, dry THF ( $5 \mathrm{~mL}$ ), and $\mathrm{LiAlH}_{4} 1 \mathrm{M}$ in THF $(1.2 \mathrm{~mL}, 1.20 \mathrm{mmol})$ were used to produce 22b. Purification : Heptane/EtOAc (7:3). White amorphous solid (54.8 mg, 58\%).

H.R.M.S. (I.C., $\mathrm{m} / \mathrm{z}$ ) calcd for $\mathrm{C}_{24} \mathrm{H}_{29} \mathrm{~N}_{2} \mathrm{O}_{3}{ }^{+}\left(\mathrm{MH}^{+}\right)$393.2178, found : 393.2165 .

I.R. $\left(\mathrm{CHCl}_{3}\right) \vee\left(\mathrm{cm}^{-1}\right)$ : $3352(\mathrm{~N}-\mathrm{H}), 1497$ (C-Car), 1196 (Car-O), 1159 (C-O).

M. S. (E.S.I., $\mathrm{m} / \mathrm{z}): 393\left(\mathrm{MH}^{+}\right)$.

1H RMN $\left(\mathrm{CDCl}_{3}, 300 \mathrm{MHz}\right) \delta(\mathrm{ppm}): 7.40(2 \mathrm{H}, \mathrm{d}, \mathrm{J}=7.6 \mathrm{~Hz}, \mathrm{Har}(\mathrm{Bn})), 7.30(2 \mathrm{H}, \mathrm{t}, \mathrm{J}$ $=7.6 \mathrm{~Hz}, \operatorname{Har}(\mathrm{Bn})), 7.23(1 \mathrm{H}, \mathrm{m}, \operatorname{Har}(\mathrm{Bn})), 6.97\left(1 \mathrm{H}, \mathrm{d}, \mathrm{J}=8.2 \mathrm{~Hz}, \mathrm{H}_{11}\right), 6.31(1 \mathrm{H}$, dd, J = 8.2 Hz, J = 2.2 Hz, H10), $6.25\left(1 \mathrm{H}, \mathrm{d}, \mathrm{J}=2.2 \mathrm{~Hz}, \mathrm{H}_{8}\right), 4.05(1 \mathrm{H}, \mathrm{d}, \mathrm{J}=12.6 \mathrm{~Hz}$, $\left.\mathrm{CH}_{2}(\mathrm{Bn})\right), 4.00-3.85$ (4H, m, Hdioxolane), $3.78\left(1 \mathrm{H}, \mathrm{t}, \mathrm{J}=4.2 \mathrm{~Hz}, \mathrm{H}_{6 \mathrm{a}}\right), 3.75(3 \mathrm{H}, \mathrm{s}$, $\left.\mathrm{OCH}_{3}\right), 3.13\left(1 \mathrm{H}, \mathrm{d}, \mathrm{J}=12.6 \mathrm{~Hz}, \mathrm{CH}_{2}(\mathrm{Bn})\right), 2.90\left(1 \mathrm{H}, \mathrm{dt}, \mathrm{J}=8.9 \mathrm{~Hz}, \mathrm{~J}=2.0 \mathrm{~Hz}, \mathrm{H}_{2}\right)$, $2.50\left(1 \mathrm{H}, \mathrm{t}, \mathrm{J}=3.6 \mathrm{~Hz}, \mathrm{H}_{3 \mathrm{a}}\right), 2.43\left(1 \mathrm{H}, \mathrm{dd}, \mathrm{J}=3.6 \mathrm{~Hz}, \mathrm{~J}=14.6 \mathrm{~Hz}, \mathrm{H}_{4}\right), 2.34(1 \mathrm{H}, \mathrm{q}, \mathrm{J}=$ $\left.8.9 \mathrm{~Hz}, \mathrm{H}_{2}\right), 2.11\left(1 \mathrm{H}, \mathrm{ddd}, \mathrm{J}=13.2 \mathrm{~Hz}, \mathrm{~J}=8.2 \mathrm{~Hz}, \mathrm{~J}=2.0 \mathrm{~Hz}, \mathrm{H}_{1}\right), 2.00-1.96(2 \mathrm{H}, \mathrm{m}$, $\mathrm{H}_{4}$ and $\left.\mathrm{H}_{6}\right), 1.87-1.80\left(2 \mathrm{H}, \mathrm{m}, \mathrm{H}_{1}\right.$ and $\left.\mathrm{H}_{6}\right)$.

${ }^{13} \mathbf{C}$ RMN $\left(\mathrm{CDCl}_{3}, 75 \mathrm{MHz}\right) \delta(\mathrm{ppm}): 160.2\left(\mathrm{C}_{9}\right), 151.4\left(\mathrm{C}_{7 \mathrm{a}}\right), 139.8(\mathrm{Car}(\mathrm{Bn})), 129.1$ $\left(\mathrm{C}_{11 \mathrm{a}}\right.$ and $\left.\mathrm{Car} \mathrm{Bn}\right), 128.2$ (Car (Bn)), $126.8(\mathrm{Car}(\mathrm{Bn})), 123.8\left(\mathrm{C}_{11}\right), 108.2\left(\mathrm{C}_{5}\right), 104.4$ $\left(\mathrm{C}_{10}\right), 96.8\left(\mathrm{C}_{8}\right), 68.7\left(\mathrm{C}_{3 \mathrm{a}}\right), 65.7\left(\mathrm{C}_{6 \mathrm{a}}\right), 64.1$ and 63.9 (Cdioxolane), $56.7\left(\mathrm{CH}_{2}(\mathrm{Bn})\right)$, $55.4\left(\mathrm{OCH}_{3}\right), 52.4\left(\mathrm{C}_{11 \mathrm{~b}}\right), 52.2\left(\mathrm{C}_{2}\right), 37.7\left(\mathrm{C}_{1}\right), 35.4\left(\mathrm{C}_{4}\right), 32.1\left(\mathrm{C}_{6}\right)$.

( \pm )-8-methoxy-3-benzyl-5-(1',4'-dioxaspiro[4.5])-1,2,3a,4,6,6a,7heptahydro-pyrrolo[2,3- $d$ ] carbazole 22c

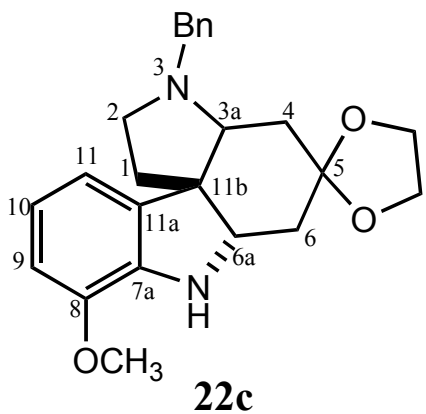

( \pm )-8-methoxy-3-benzyl-2-oxo-5-(1',4'-dioxaspiro[4.5])-1,3a,4,6,6a,7-hexahydropyrrolo [2,3- $d$ ] carbazole $21 \mathrm{c}(458.4 \mathrm{mg}, 1.13 \mathrm{mmol})$, dry THF $(22 \mathrm{~mL})$, and $\mathrm{LiAlH}_{4}$ $1 \mathrm{M}$ in THF $(5.6 \mathrm{~mL}, 5.64 \mathrm{mmol})$ were used to produce 22c. Purification: Heptane/EtOAc (7:3). White amorphous solid (339.2 mg, 77\%).

H.R.M.S. (I.C., $\mathrm{m} / \mathrm{z}$ ) calcd for $\mathrm{C}_{24} \mathrm{H}_{29} \mathrm{~N}_{2} \mathrm{O}_{3}{ }^{+}\left(\mathrm{MH}^{+}\right)$393.2178, found : 393.2148 . 
I.R. $\left(\mathrm{CHCl}_{3}\right) \vee\left(\mathrm{cm}^{-1}\right)$ : $3331(\mathrm{~N}-\mathrm{H}), 1493$ (C-Car), 1240 (Car-O), 1044 (C-O).

M. S. (E.S.I., $\mathrm{m} / \mathrm{z}): 393\left(\mathrm{MH}^{+}\right)$.

1H RMN $\left(\mathrm{CDCl}_{3}, 500 \mathrm{MHz}\right) \delta(\mathrm{ppm}): 7.39(2 \mathrm{H}, \mathrm{d}, \mathrm{J}=7.6 \mathrm{~Hz}, \operatorname{Har}(\mathrm{Bn})), 7.31(2 \mathrm{H}, \mathrm{t}$, $\mathrm{J}=8.2 \mathrm{~Hz}, \mathrm{~J}=7.6 \mathrm{~Hz}$, Har (Bn)), $7.25(1 \mathrm{H}, \mathrm{d}, \mathrm{J}=8.9 \mathrm{~Hz}, \operatorname{Har}(\mathrm{Bn})), 6.78\left(2 \mathrm{H}, \mathrm{m}, \mathrm{H}_{9}\right.$ and $\left.\mathrm{H}_{10}\right), 6.67\left(1 \mathrm{H}, \mathrm{m}, \mathrm{H}_{11}\right), 4.06\left(1 \mathrm{H}, \mathrm{d}, \mathrm{J}=12.2 \mathrm{~Hz}, \mathrm{CH}_{2}(\mathrm{Bn})\right), 3.98(1 \mathrm{H}, \mathrm{dd}, \mathrm{J}=3.7$ $\left.\mathrm{Hz}, \mathrm{J}=8.2 \mathrm{~Hz}, \mathrm{H}_{6 \mathrm{a}}\right), 3.90\left(4 \mathrm{H}, \mathrm{m}\right.$, Hdioxolane), $3.80\left(3 \mathrm{H}, \mathrm{s}, \mathrm{OCH}_{3}\right), 3.22(1 \mathrm{H}, \mathrm{d}, \mathrm{J}=$ $\left.12.5 \mathrm{~Hz}, \mathrm{CH}_{2}(\mathrm{Bn})\right), 2.91\left(1 \mathrm{H}, \mathrm{t}, \mathrm{J}=7.6 \mathrm{~Hz}, \mathrm{H}_{2}\right), 2.65\left(1 \mathrm{H}, \mathrm{sl}, \mathrm{H}_{3 \mathrm{a}}\right), 2.41(1 \mathrm{H}, \mathrm{t}, \mathrm{J}=8.2$ $\left.\mathrm{Hz}, \mathrm{H}_{2}\right), 2.35\left(1 \mathrm{H}, \mathrm{dd}, \mathrm{J}=4.0 \mathrm{~Hz}, \mathrm{~J}=14.6 \mathrm{~Hz}, \mathrm{H}_{6}\right), 2.11(1 \mathrm{H}, \mathrm{ddd}, \mathrm{J}=1.8 \mathrm{~Hz}, \mathrm{~J}=8.2$ $\left.\mathrm{Hz}, \mathrm{J}=13.1 \mathrm{~Hz}, \mathrm{H}_{1}\right), 1.98\left(2 \mathrm{H}, \mathrm{m}, \mathrm{H}_{4}\right.$ and $\left.\mathrm{H}_{6}\right), 1.87\left(2 \mathrm{H}, \mathrm{m}, \mathrm{H}_{1}\right.$ and $\left.\mathrm{H}_{4}\right)$.

${ }^{13} \mathbf{C}$ RMN $\left(\mathrm{CDCl}_{3}, 75 \mathrm{MHz}\right) \delta(\mathrm{ppm}): 145.8\left(\mathrm{C}_{8}\right), 139.6(\mathrm{Car}(\mathrm{Bn})), 138.9\left(\mathrm{C}_{7 \mathrm{a}}\right), 137.6$ $\left(\mathrm{C}_{11 \mathrm{a}}\right), 129.0$ (Car (Bn)), 128.1 (Car (Bn)), 126.7 (Car (Bn)), $119.8\left(\mathrm{C}_{10}\right), 115.7\left(\mathrm{C}_{11}\right)$, $109.4\left(\mathrm{C}_{9}\right), 108.1\left(\mathrm{C}_{5}\right), 67.7\left(\mathrm{C}_{3 \mathrm{a}}\right), 65.3\left(\mathrm{C}_{6 \mathrm{a}}\right), 63.9$ (Cdioxolane), $56.7\left(\mathrm{CH}_{2}(\mathrm{Bn})\right), 55.2$ $\left(\mathrm{OCH}_{3}\right), 53.9\left(\mathrm{C}_{11 \mathrm{~b}}\right), 52.0\left(\mathrm{C}_{2}\right), 37.4\left(\mathrm{C}_{1}\right), 35.6\left(\mathrm{C}_{6}\right), 32.4\left(\mathrm{C}_{4}\right)$.

\section{General Procedure for the Synthesis of Carbazole 23a-c}

To a spatula of Ni Raney dissolved in EtOH, 22a-c was added. After being stirred at room temperature for 18 hours under $\mathrm{H}_{2}$ pressure, the reaction mixture was filtered with celite, washed with $\mathrm{MeOH}$ and evaporated. The purification by preparative TLC plates of the residue afforded of the desired product, 23a-c, and the starting product, 22a-c, as indicated below for each case.

\section{(土)-5-(1',4'-dioxaspiro[4.5])-1,2,3,3a,4,6,6a,7- octahydro-pyrrolo[2,3- $d]$ carbazole 23a}

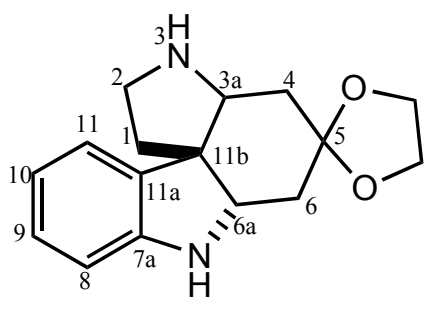

23a

( \pm )-3-benzyl-5-(1',4'-dioxaspiro[4.5])-1,2,3a,4,6,6a,7-heptahydro-pyrrolo[2,3-d] carbazole 22a (53.7 mg, $0.15 \mathrm{mmol})$, a spatula of Ni Raney, and EtOH (5 mL) were used to produce 23a. Purification : $\mathrm{CH}_{2} \mathrm{Cl}_{2} / \mathrm{MeOH}$ (9:1). Yellow amorphous solid (22.9 $\mathrm{mg}, 57 \%$ ). $7.6 \mathrm{mg}$ of $\mathbf{2 2 a}$ were recovered. 
H.R.M.S. (I.C., $\mathrm{m} / \mathrm{z}$ ) calcd for $\mathrm{C}_{16} \mathrm{H}_{21} \mathrm{~N}_{2} \mathrm{O}_{3}{ }^{+}\left(\mathrm{MH}^{+}\right)$273.1603, found : 273.1602 .

I.R. $\left(\mathrm{CHCl}_{3}\right) \vee\left(\mathrm{cm}^{-1}\right): 3345(\mathrm{~N}-\mathrm{H}), 1483$ and 1463 (C-Car), 1046 (C-O).

M. S. (E.S.I., $\mathrm{m} / \mathrm{z}): 273\left(\mathrm{MH}^{+}\right)$.

1H RMN $\left(\mathrm{CDCl}_{3}, 300 \mathrm{MHz}\right) \delta(\mathrm{ppm}): 7.07\left(1 \mathrm{H}, \mathrm{dd}, \mathrm{J}=7.3 \mathrm{~Hz}, \mathrm{~J}=7.6 \mathrm{~Hz}, \mathrm{H}_{9}\right.$ and $\left.\mathrm{H}_{11}\right), 6.69\left(1 \mathrm{H}, \mathrm{d}, \mathrm{J}=7.6 \mathrm{~Hz}, \mathrm{H}_{8}\right), 4.03-3.91$ (4H, m, Hdioxolane), 3.84 (2H, sl, NH), 3.79-3.74 (2H, m, $\mathrm{H}_{3 \mathrm{a}}$ and $\left.\mathrm{H}_{6 \mathrm{a}}\right), 3.28-3.12\left(2 \mathrm{H}, \mathrm{m}, \mathrm{H}_{2}\right), 2.34(1 \mathrm{H}, \mathrm{ddd}, \mathrm{J}=12.9 \mathrm{~Hz}, \mathrm{~J}=$ $\left.8.2 \mathrm{~Hz}, \mathrm{~J}=4.7 \mathrm{~Hz}, \mathrm{H}_{1}\right), 2.06-2.00\left(1 \mathrm{H}, \mathrm{m}, \mathrm{H}_{4}\right), 1.92-1.84\left(3 \mathrm{H}, \mathrm{m}, \mathrm{H}_{1}, \mathrm{H}_{4}\right.$ and $\left.\mathrm{H}_{6}\right), 1.69$ $\left(1 \mathrm{H}, \mathrm{dd}, \mathrm{J}=13.2 \mathrm{~Hz}, \mathrm{~J}=9.5 \mathrm{~Hz}, \mathrm{H}_{6}\right)$.

${ }^{13} \mathbf{C ~ R M N ~}\left(\mathrm{CDCl}_{3}, 75 \mathrm{MHz}\right) \delta(\mathrm{ppm}): 149.8\left(\mathrm{C}_{7 \mathrm{a}}\right), 132.5\left(\mathrm{C}_{11 \mathrm{a}}\right), 128.2\left(\mathrm{C}_{9}\right), 122.2$ $\left(\mathrm{C}_{11}\right), 119.7\left(\mathrm{C}_{10}\right), 110.8\left(\mathrm{C}_{8}\right), 107.9\left(\mathrm{C}_{5}\right), 64.2$ and 64.8 (Cdioxolane), 62.7 and 60.7 $\left(\mathrm{C}_{3 \mathrm{a}}\right.$ and $\left.\mathrm{C}_{6 \mathrm{a}}\right), 53.7\left(\mathrm{C}_{11 \mathrm{~b}}\right), 44.2\left(\mathrm{C}_{2}\right), 39.1\left(\mathrm{C}_{1}\right), 38.0\left(\mathrm{C}_{6}\right), 33.3\left(\mathrm{C}_{4}\right)$.

(士)-9-methoxy-5-(1',4'-dioxaspiro[4.5])-1,2,3,3a,4,6,6a,7octahydro-pyrrolo $[2,3-d]$ carbazole 23b

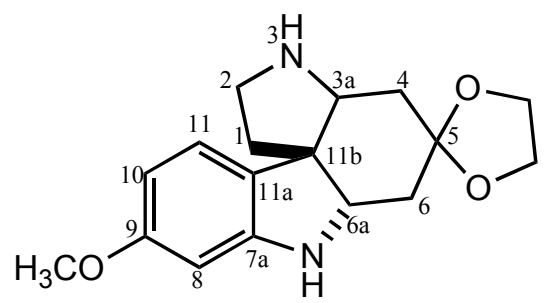

23b

(土)-9-methoxy-3-benzyl-5-(1',4'-dioxaspiro[4.5])-1,2,3a,4,6,6a,7-heptahydropyrrolo[2,3- $d$ ] carbazole $22 \mathbf{b}(63.3 \mathrm{mg}, 0.16 \mathrm{mmol})$, a spatula of Ni Raney, and EtOH (5 mL) were used to produce 23b. Purification: $\mathrm{CH}_{2} \mathrm{Cl}_{2} / \mathrm{MeOH}(9: 1)$. Yellow amorphous solid (29.4 mg, $60 \%) .17 .1 \mathrm{mg}$ of $\mathbf{2 2} \mathbf{b}$ were recovered.

H.R.M.S. (I.C., $\mathrm{m} / \mathrm{z}$ ) calcd for $\mathrm{C}_{17} \mathrm{H}_{23} \mathrm{~N}_{2} \mathrm{O}_{3}{ }^{+}\left(\mathrm{MH}^{+}\right)$303.1709, found : 303.1695 .

I.R. $\left(\mathrm{CHCl}_{3}\right) \vee\left(\mathrm{cm}^{-1}\right): 3341(\mathrm{~N}-\mathrm{H}), 1498$ (C-Car), 1269 (Car-O), 1091 (C-O).

M. S. (E.S.I., m/z) : $303\left(\mathrm{MH}^{+}\right)$.

1H RMN $\left(\mathrm{CDCl}_{3}, 500 \mathrm{MHz}\right) \delta(\mathrm{ppm}): 6.94\left(1 \mathrm{H}, \mathrm{d}, \mathrm{J}=8.2 \mathrm{~Hz}, \mathrm{H}_{11}\right), 6.31(1 \mathrm{H}, \mathrm{d}, \mathrm{J}=$ $\left.8.2 \mathrm{~Hz}, \mathrm{H}_{10}\right), 6.27\left(1 \mathrm{H}, \mathrm{s}, \mathrm{H}_{8}\right), 4.00-3.90$ (4H, m, Hdioxolane), 3.75 (4H, sl, $\mathrm{OCH}_{3}$ and $\left.\mathrm{H}_{6 \mathrm{a}}\right), 3.61\left(1 \mathrm{H}, \mathrm{sl}, \mathrm{H}_{3 \mathrm{a}}\right), 3.46(2 \mathrm{H}, \mathrm{sl}, 2 \mathrm{x} \mathrm{NH}), 3.19\left(1 \mathrm{H}, \mathrm{m}, \mathrm{H}_{2}\right), 3.11\left(1 \mathrm{H}, \mathrm{m}, \mathrm{H}_{2}\right), 2.28$ $\left(1 \mathrm{H}, \mathrm{ddd}, \mathrm{J}=13.2 \mathrm{~Hz}, \mathrm{~J}=8.2 \mathrm{~Hz}, \mathrm{~J}=4.8 \mathrm{~Hz}, \mathrm{H}_{1}\right), 1.97\left(1 \mathrm{H}, \mathrm{d}, \mathrm{J}=14.5 \mathrm{~Hz}, \mathrm{H}_{4}\right), 1.91$ $1.82\left(3 \mathrm{H}, \mathrm{m}, \mathrm{H}_{1}, \mathrm{H}_{4}\right.$ and $\left.\mathrm{H}_{6}\right), 1.70\left(1 \mathrm{H}, \mathrm{dd}, \mathrm{J}=13.6 \mathrm{~Hz}, \mathrm{~J}=9.1 \mathrm{~Hz}, \mathrm{H}_{6}\right)$. 
${ }^{13} \mathbf{C}$ RMN $\left(\mathrm{CDCl}_{3}, 75 \mathrm{MHz}\right) \delta(\mathrm{ppm})$ : $160.4\left(\mathrm{C}_{9}\right), 151.1\left(\mathrm{C}_{7 \mathrm{a}}\right), 125.6\left(\mathrm{C}_{11 \mathrm{a}}\right), 122.4$ $\left(\mathrm{C}_{11}\right), 108.0\left(\mathrm{C}_{5}\right), 104.2\left(\mathrm{C}_{10}\right), 97.6\left(\mathrm{C}_{8}\right), 64.1$ and 64.7 (Cdioxolane), $63.3\left(\mathrm{C}_{6 \mathrm{a}}\right), 60.9$ $\left(\mathrm{C}_{3 \mathrm{a}}\right), 55.4\left(\mathrm{OCH}_{3}\right), 53.1\left(\mathrm{C}_{11 \mathrm{~b}}\right), 44.4\left(\mathrm{C}_{2}\right), 39.5\left(\mathrm{C}_{1}\right), 38.0\left(\mathrm{C}_{6}\right), 33.6\left(\mathrm{C}_{4}\right)$.

( \pm )-8-methoxy-5-(1',4'-dioxaspiro[4.5])-1,2,3,3a,4,6,6a,7octahydro-pyrrolo $[2,3-d]$ carbazole 23c

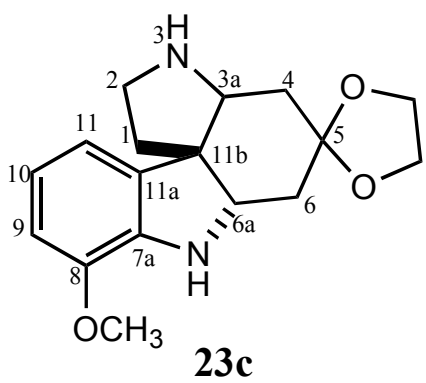

( \pm )-8-methoxy-3-benzyl-5-(1',4'-dioxaspiro[4.5])-1,2,3a,4,6,6a,7-heptahydropyrrolo[2,3-d] carbazole 22c (36.6 mg, $0.09 \mathrm{mmol}$ ), a spatula of Ni Raney, and EtOH (5 mL) were used to produce 23c. Purification: $\mathrm{CH}_{2} \mathrm{Cl}_{2} / \mathrm{MeOH}(9: 1)$. Yellow amorphous solid (16.5 mg, 58\%). $14.1 \mathrm{mg}$ of 22c were recovered.

H.R.M.S. (I.C., m/z) calcd for $\mathrm{C}_{17} \mathrm{H}_{23} \mathrm{~N}_{2} \mathrm{O}_{3}{ }^{+}\left(\mathrm{MH}^{+}\right)$303.1708, found : 303.1727 .

I.R. $\left(\mathrm{CHCl}_{3}\right) \vee\left(\mathrm{cm}^{-1}\right): 3342(\mathrm{~N}-\mathrm{H}), 1488$ (C-Car), 1257 (Car-O), 1035 (C-O).

M. S. (E.S.I., $\mathrm{m} / \mathrm{z}): 325\left(\mathrm{MNa}^{+}\right), 303\left(\mathrm{MH}^{+}\right)$.

1H RMN $\left(\mathrm{CDCl}_{3}, 500 \mathrm{MHz}\right) \delta(\mathrm{ppm}): 6.79\left(1 \mathrm{H}, \mathrm{t}, \mathrm{J}=7.9 \mathrm{~Hz}, \mathrm{~J}=7.6 \mathrm{~Hz}, \mathrm{H}_{10}\right), 6.75$ $\left(1 \mathrm{H}, \mathrm{d}, \mathrm{J}=7.2 \mathrm{~Hz}, \mathrm{H}_{11}\right), 6.71\left(1 \mathrm{H}, \mathrm{d}, \mathrm{J}=7.9 \mathrm{~Hz}, \mathrm{H}_{9}\right), 4.03-3.89$ (4H, m, Hdioxolane), $3.82\left(3 \mathrm{H}, \mathrm{s}, \mathrm{OCH}_{3}\right), 3.79\left(1 \mathrm{H}, \mathrm{dd}, \mathrm{J}=2.7 \mathrm{~Hz}, \mathrm{~J}=5.2 \mathrm{~Hz}, \mathrm{H}_{3 \mathrm{a}}\right), 3.78(1 \mathrm{H}, \mathrm{dd}, \mathrm{J}=5.5 \mathrm{~Hz}$, $\left.\mathrm{J}=10.1 \mathrm{~Hz}, \mathrm{H}_{6 \mathrm{a}}\right), 3.64(2 \mathrm{H}, \mathrm{sl}, \mathrm{NH}), 3.21\left(2 \mathrm{H}, \mathrm{m}, \mathrm{H}_{2}\right), 2.39(1 \mathrm{H}, \mathrm{ddd}, \mathrm{J}=4.3 \mathrm{~Hz}, \mathrm{~J}=$ $\left.8.2 \mathrm{~Hz}, \mathrm{~J}=12.8 \mathrm{~Hz}, \mathrm{H}_{1}\right), 2.04\left(1 \mathrm{H}, \mathrm{dd}, \mathrm{J}=2.1 \mathrm{~Hz}, \mathrm{~J}=16.5 \mathrm{~Hz}, \mathrm{H}_{4}\right), 1.87\left(3 \mathrm{H}, \mathrm{m}, \mathrm{H}_{1}\right.$, $\mathrm{H}_{4}$ and $\left.\mathrm{H}_{6}\right), 1.65\left(1 \mathrm{H}, \mathrm{dd}, \mathrm{J}=10.1 \mathrm{~Hz}, \mathrm{~J}=13.4 \mathrm{~Hz}, \mathrm{H}_{6}\right)$.

${ }^{13} \mathrm{C}$ RMN $\left(\mathrm{CDCl}_{3}, 75 \mathrm{MHz}\right) \delta(\mathrm{ppm}): 146.2\left(\mathrm{C}_{8}\right), 138.5\left(\mathrm{C}_{7 \mathrm{a}}\right), 133.0\left(\mathrm{C}_{11 \mathrm{a}}\right), 120.3$ $\left(\mathrm{C}_{10}\right), 114.4\left(\mathrm{C}_{11}\right), 110.0\left(\mathrm{C}_{9}\right), 107.8\left(\mathrm{C}_{5}\right), 64.4$ (Cdioxolane), $62.9\left(\mathrm{C}_{6 \mathrm{a}}\right), 60.4\left(\mathrm{C}_{3 \mathrm{a}}\right)$, $55.3\left(\mathrm{OCH}_{3}\right), 54.3\left(\mathrm{C}_{11 \mathrm{~b}}\right), 43.9\left(\mathrm{C}_{2}\right), 38.9\left(\mathrm{C}_{1}\right), 38.1\left(\mathrm{C}_{6}\right), 33.1\left(\mathrm{C}_{4}\right)$.

\section{General Procedure for the Synthesis of Carbazole 8a-c}


To 23a-c dissolved in THF, $\mathrm{HCl} 35 \% \mathrm{H}_{2} \mathrm{O}$ was added at $0^{\circ} \mathrm{C}$. After being stirred at room temperature for 24 hours, the reaction mixture was extracted with $\mathrm{CH}_{2} \mathrm{Cl}_{2}$. The combined organic layer was washed with $\mathrm{NaOH} 1 \mathrm{M}, \mathrm{H}_{2} \mathrm{O}$, brine, dried $\left(\mathrm{MgSO}_{4}\right)$ and evaporated. The purification by preparative TLC plates of the residue afforded 8a-c as indicated below for each case.

( \pm )-5-oxo-1,2,3,3a,4,6,6a,7-octahydro-pyrrolo[2,3- $d]$ carbazole 8a

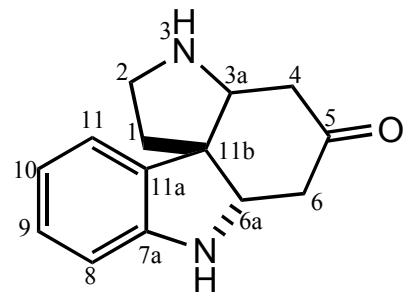

8a

(土)-5-(1',4'-dioxaspiro[4.5])-1,2,3a,4,6,6a,7-heptahydro-pyrrolo[2,3- $d$ ] carbazole 23a (20.3 mg, $0.07 \mathrm{mmol})$, THF $(1 \mathrm{~mL})$, and $\mathrm{HCl} \mathrm{35 \%} \mathrm{H}_{2} \mathrm{O}(1 \mathrm{~mL})$ were used to produce 8a. Purification : $\mathrm{CH}_{2} \mathrm{Cl}_{2} / \mathrm{MeOH}$ (9:1). Yellow amorphous solid (8.8 mg, 52\%).

H.R.M.S. (I.C., $\mathrm{m} / \mathrm{z})$ calcd for $\mathrm{C}_{14} \mathrm{H}_{17} \mathrm{~N}_{2} \mathrm{O}_{2}{ }^{+}\left(\mathrm{MH}^{+}\right)$229.1341, found : 229.1314 .

I.R. $\left(\mathrm{CHCl}_{3}\right) \vee\left(\mathrm{cm}^{-1}\right)$ : $3350(\mathrm{~N}-\mathrm{H}), 1708(\mathrm{C}=\mathrm{O}), 1483$ (C-Car), 1259 (Car-O).

M. S. (E.S.I., m/z) : $229\left(\mathrm{MH}^{+}\right)$.

1H RMN $\left(\mathrm{CDCl}_{3}, 500 \mathrm{MHz}\right) \delta(\mathrm{ppm}): 7.14\left(1 \mathrm{H}, \mathrm{d}, \mathrm{J}=7.3 \mathrm{~Hz}, \mathrm{H}_{11}\right), 7.07(1 \mathrm{H}, \mathrm{t}, \mathrm{J}=7.3$

$\left.\mathrm{Hz}, \mathrm{H}_{9}\right), 6.80\left(1 \mathrm{H}, \mathrm{t}, \mathrm{J}=7.3 \mathrm{~Hz}, \mathrm{H}_{10}\right), 6.62\left(1 \mathrm{H}, \mathrm{d}, \mathrm{J}=7.3 \mathrm{~Hz}, \mathrm{H}_{8}\right), 4.03(1 \mathrm{H}, \mathrm{t}, \mathrm{J}=3.2$ $\left.\mathrm{Hz}, \mathrm{H}_{6 \mathrm{a}}\right), 3.29\left(1 \mathrm{H}, \mathrm{t}, \mathrm{J}=3.2 \mathrm{~Hz}, \mathrm{H}_{3 \mathrm{a}}\right), 3.25\left(1 \mathrm{H}, \mathrm{dd}, \mathrm{J}=9.0 \mathrm{~Hz}, \mathrm{~J}=3.5 \mathrm{~Hz}, \mathrm{H}_{2}\right), 3.15$ $\left(1 \mathrm{H}, \mathrm{q}, \mathrm{J}=9.0 \mathrm{~Hz}, \mathrm{H}_{2}\right), 2.96\left(1 \mathrm{H}, \mathrm{dd}, \mathrm{J}=17.7 \mathrm{~Hz}, \mathrm{~J}=3.2 \mathrm{~Hz}, \mathrm{H}_{6}\right), 2.57(1 \mathrm{H}, \mathrm{dd}, \mathrm{J}=$ $\left.17.3 \mathrm{~Hz}, \mathrm{~J}=3.2 \mathrm{~Hz}, \mathrm{H}_{4}\right), 2.55\left(1 \mathrm{H}, \mathrm{dd}, \mathrm{J}=17.7 \mathrm{~Hz}, \mathrm{~J}=3.2 \mathrm{~Hz}, \mathrm{H}_{6}\right), 2.51(1 \mathrm{H}, \mathrm{dd}, \mathrm{J}=$ $\left.8.5 \mathrm{~Hz}, \mathrm{~J}=3.5 \mathrm{~Hz}, \mathrm{H}_{1}\right), 2.42\left(1 \mathrm{H}, \mathrm{dd}, \mathrm{J}=17.3 \mathrm{~Hz}, \mathrm{~J}=3.2 \mathrm{~Hz}, \mathrm{H}_{4}\right), 1.99\left(1 \mathrm{H}, \mathrm{m}, \mathrm{H}_{1}\right)$.

${ }^{13} \mathrm{C}$ RMN $\left(\mathrm{CDCl}_{3}, 125 \mathrm{MHz}\right) \delta(\mathrm{ppm}): 210.2\left(\mathrm{C}_{5}\right), 150.3\left(\mathrm{C}_{7 \mathrm{a}}\right), 134.7\left(\mathrm{C}_{11 \mathrm{a}}\right), 128.2$ $\left(\mathrm{C}_{9}\right), 123.2\left(\mathrm{C}_{11}\right), 119.6\left(\mathrm{C}_{10}\right), 109.6\left(\mathrm{C}_{8}\right), 66.1\left(\mathrm{C}_{3 \mathrm{a}}\right), 65.6\left(\mathrm{C}_{6 \mathrm{a}}\right), 51.4\left(\mathrm{C}_{11 \mathrm{~b}}\right), 46.3\left(\mathrm{C}_{2}\right)$, $41.2\left(\mathrm{C}_{4}\right), 40.5\left(\mathrm{C}_{1}\right), 40.2\left(\mathrm{C}_{6}\right)$.

( \pm )-9-methoxy-5-oxo-1,2,3,3a,4,6,6a,7-octahydro-pyrrolo[2,3-d]carbazole $8 \mathbf{b}$ 


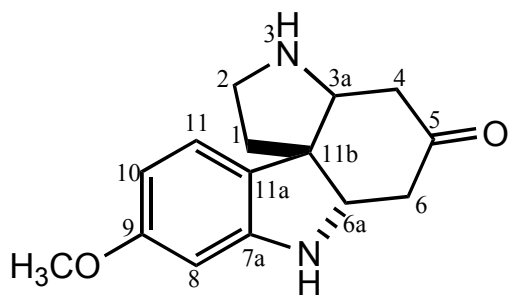

8b

(土)-9-methoxy-5-(1',4'-dioxaspiro[4.5])-1,2,3a,4,6,6a,7-heptahydro-pyrrolo[2,3-d] carbazole 23b $(27.4 \mathrm{mg}, 0.09 \mathrm{mmol})$, THF $(1 \mathrm{~mL})$, and $\mathrm{HCl} 35 \% \mathrm{H}_{2} \mathrm{O}(1 \mathrm{~mL})$ were used to produce 8b. Purification : $\mathrm{CH}_{2} \mathrm{Cl}_{2} / \mathrm{MeOH}$ (9:1). Yellow amorphous solid (7.5 $\mathrm{mg}, 32 \%)$.

H.R.M.S. (I.C., m/z) calcd for $\mathrm{C}_{15} \mathrm{H}_{19} \mathrm{~N}_{2} \mathrm{O}_{2}{ }^{+}\left(\mathrm{MH}^{+}\right)$259.1447, found : 259.1434.

I.R. $\left(\mathrm{CHCl}_{3}\right) \vee\left(\mathrm{cm}^{-1}\right): 3358(\mathrm{~N}-\mathrm{H}), 1709(\mathrm{C}=\mathrm{O}), 1500$ (C-Car), 1218 (Car-O).

M. S. (E.S.I., m/z) : $281\left(\mathrm{MNa}^{+}\right), 259\left(\mathrm{MH}^{+}\right)$.

1H RMN $\left(\mathrm{CDCl}_{3}, 500 \mathrm{MHz}\right) \delta(\mathrm{ppm}): 7.01\left(1 \mathrm{H}, \mathrm{d}, \mathrm{J}=8.2 \mathrm{~Hz}, \mathrm{H}_{11}\right), 6.34(1 \mathrm{H}, \mathrm{dd}, \mathrm{J}=$ $\left.8.2 \mathrm{~Hz}, \mathrm{~J}=2.2 \mathrm{~Hz}, \mathrm{H}_{10}\right), 6.20\left(1 \mathrm{H}, \mathrm{d}, \mathrm{J}=2.2 \mathrm{~Hz}, \mathrm{H}_{8}\right), 4.03\left(1 \mathrm{H}, \mathrm{s}, \mathrm{H}_{6 \mathrm{a}}\right), 3.76(3 \mathrm{H}, \mathrm{s}$, $\left.\mathrm{OCH}_{3}\right), 3.26\left(1 \mathrm{H}, \mathrm{s}, \mathrm{H}_{3 \mathrm{a}}\right), 3.23\left(1 \mathrm{H}, \mathrm{d}, \mathrm{J}=8.2 \mathrm{~Hz}, \mathrm{~J}=3.8 \mathrm{~Hz}, \mathrm{H}_{2}\right), 3.13(1 \mathrm{H}, \mathrm{q}, \mathrm{J}=8.2$ $\left.\mathrm{Hz}, \mathrm{H}_{2}\right), 2.95\left(1 \mathrm{H}, \mathrm{dd}, \mathrm{J}=17.3 \mathrm{~Hz}, \mathrm{~J}=3.2 \mathrm{~Hz}, \mathrm{H}_{6}\right), 2.55(1 \mathrm{H}, \mathrm{dd}, \mathrm{J}=17.3 \mathrm{~Hz}, \mathrm{~J}=3.2$ $\left.\mathrm{Hz}, \mathrm{H}_{6}\right), 2.53\left(1 \mathrm{H}, \mathrm{dd}, \mathrm{J}=17.3 \mathrm{~Hz}, \mathrm{~J}=2.8 \mathrm{~Hz}, \mathrm{H}_{4}\right), 2.47(1 \mathrm{H}, \mathrm{tt}, \mathrm{J}=9.3 \mathrm{~Hz}, \mathrm{~J}=4.6 \mathrm{~Hz}$, $\left.\mathrm{H}_{1}\right), 2.41\left(1 \mathrm{H}, \mathrm{dd}, \mathrm{J}=17.3 \mathrm{~Hz}, \mathrm{~J}=2.8 \mathrm{~Hz}, \mathrm{H}_{4}\right), 1.97\left(1 \mathrm{H}, \mathrm{m}, \mathrm{H}_{1}\right), 1.79(2 \mathrm{H}, \mathrm{sl}, 2 \mathrm{x} \mathrm{NH})$. ${ }^{13} \mathbf{C ~ R M N}\left(\mathrm{CDCl}_{3}, 125 \mathrm{MHz}\right) \delta(\mathrm{ppm}): 210.2\left(\mathrm{C}_{5}\right), 160.6\left(\mathrm{C}_{9}\right), 151.6\left(\mathrm{C}_{7 \mathrm{a}}\right), 126.9$ $\left(\mathrm{C}_{11 \mathrm{a}}\right), 123.6\left(\mathrm{C}_{11}\right), 104.7\left(\mathrm{C}_{10}\right), 96.1\left(\mathrm{C}_{8}\right), 66.3$ and $66.1\left(\mathrm{C}_{3 \mathrm{a}}\right.$ and $\left.\mathrm{C}_{6 \mathrm{a}}\right), 55.5\left(\mathrm{OCH}_{3}\right)$, $50.8\left(\mathrm{C}_{11 \mathrm{~b}}\right), 46.2\left(\mathrm{C}_{2}\right), 41.1-40.5-40.4\left(\mathrm{C}_{1}-\mathrm{C}_{4}-\mathrm{C}_{6}\right)$.

( \pm )-8-methoxy-5-oxo-1,2,3,3a,4,6,6a,7-octahydro-pyrrolo[2,3- $d]$ carbazole 8c

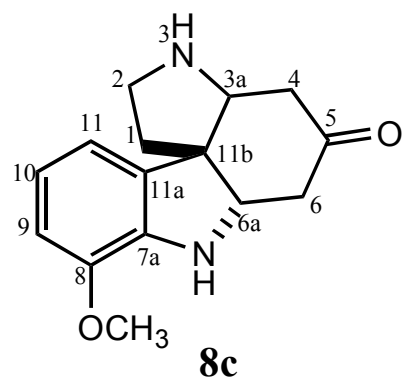

( \pm )-8-methoxy-5-(1',4'-dioxaspiro[4.5])-1,2,3a,4,6,6a,7-heptahydro-pyrrolo[2,3- $d$ ] carbazole $23 \mathrm{c}(32.8 \mathrm{mg}, 0.11 \mathrm{mmol})$, THF $(1 \mathrm{~mL})$, and $\mathrm{HCl} 35 \% \mathrm{H}_{2} \mathrm{O}(1 \mathrm{~mL})$ were 
used to produce 8c. Purification : $\mathrm{CH}_{2} \mathrm{Cl}_{2} / \mathrm{MeOH}$ (9:1). Yellow amorphous solid (12.8 mg, 46\%).

H.R.M.S. (I.C., m/z) calcd for $\mathrm{C}_{15} \mathrm{H}_{19} \mathrm{~N}_{2} \mathrm{O}_{2}{ }^{+}\left(\mathrm{MH}^{+}\right)$259.1441, found : 259.1467.

I.R. $\left(\mathrm{CHCl}_{3}\right) \vee\left(\mathrm{cm}^{-1}\right): 3348(\mathrm{~N}-\mathrm{H}), 1710(\mathrm{C}=\mathrm{O}), 1489$ (C-Car), 1258 (Car-O).

M. S. (E.S.I., $\mathrm{m} / \mathrm{z}): 281\left(\mathrm{MNa}^{+}\right), 259\left(\mathrm{MH}^{+}\right)$.

${ }_{1} \mathbf{H ~ R M N}\left(\mathrm{CDCl}_{3}, 500 \mathrm{MHz}\right) \delta(\mathrm{ppm}): 6.73\left(2 \mathrm{H}, \mathrm{m}, \mathrm{H}_{10}\right.$ and $\left.\mathrm{H}_{11}\right), 6.63\left(1 \mathrm{H}, \mathrm{m}, \mathrm{H}_{9}\right)$, $3.97\left(1 \mathrm{H}, \mathrm{sl}, \mathrm{H}_{6 \mathrm{a}}\right), 3.75\left(3 \mathrm{H}, \mathrm{s}, \mathrm{OCH}_{3}\right), 3.28\left(1 \mathrm{H}, \mathrm{t}, \mathrm{J}=3.4 \mathrm{~Hz}, \mathrm{H}_{3 \mathrm{a}}\right), 3.21(1 \mathrm{H}, \mathrm{td}, \mathrm{J}=$ $\left.3.4 \mathrm{~Hz}, \mathrm{~J}=8.2 \mathrm{~Hz}, \mathrm{~J}=9.8 \mathrm{~Hz}, \mathrm{H}_{2}\right), 3.08\left(1 \mathrm{H}, \mathrm{q}, \mathrm{J}=8.9 \mathrm{~Hz}, \mathrm{H}_{2}\right), 2.90(1 \mathrm{H}, \mathrm{dd}, \mathrm{J}=3.4$ $\left.\mathrm{Hz}, \mathrm{J}=17.7 \mathrm{~Hz}, \mathrm{H}_{6}\right), 2.55\left(1 \mathrm{H}, \mathrm{dd}, \mathrm{J}=3.4 \mathrm{~Hz}, \mathrm{~J}=17.7 \mathrm{~Hz}, \mathrm{H}_{4}\right), 2.54(1 \mathrm{H}, \mathrm{dd}, \mathrm{J}=3.1$ $\left.\mathrm{Hz}, \mathrm{J}=17.7 \mathrm{~Hz}, \mathrm{H}_{6}\right), 2.46\left(1 \mathrm{H}, \mathrm{ddd}, \mathrm{J}=3.4 \mathrm{~Hz}, \mathrm{~J}=8.2 \mathrm{~Hz}, \mathrm{~J}=13.4 \mathrm{~Hz}, \mathrm{H}_{1}\right), 2.39(1 \mathrm{H}$, $\left.\mathrm{dd}, \mathrm{J}=3.4 \mathrm{~Hz}, \mathrm{~J}=17.4 \mathrm{~Hz}, \mathrm{H}_{4}\right), 1.93\left(1 \mathrm{H}, \mathrm{dt}, \mathrm{J}=8.2 \mathrm{~Hz}, \mathrm{~J}=13.7 \mathrm{~Hz}, \mathrm{H}_{1}\right)$.

${ }^{13} \mathbf{C}$ RMN $\left(\mathrm{CDCl}_{3}, 125 \mathrm{MHz}\right) \delta(\mathrm{ppm}): 209.5\left(\mathrm{C}_{5}\right), 145.2\left(\mathrm{C}_{8}\right), 139.4\left(\mathrm{C}_{7 \mathrm{a}}\right), 135.3$ $\left(\mathrm{C}_{11 \mathrm{a}}\right), 120.3\left(\mathrm{C}_{10}\right), 115.4\left(\mathrm{C}_{11}\right), 109.9\left(\mathrm{C}_{9}\right), 66.1\left(\mathrm{C}_{6 \mathrm{a}}\right), 65.7\left(\mathrm{C}_{3 \mathrm{a}}\right), 55.4\left(\mathrm{OCH}_{3}\right), 52.1$ $\left(\mathrm{C}_{11 \mathrm{~b}}\right), 46.3\left(\mathrm{C}_{2}\right), 41.1\left(\mathrm{C}_{4}\right), 40.2\left(\mathrm{C}_{6}\right), 40.2\left(\mathrm{C}_{1}\right)$. 

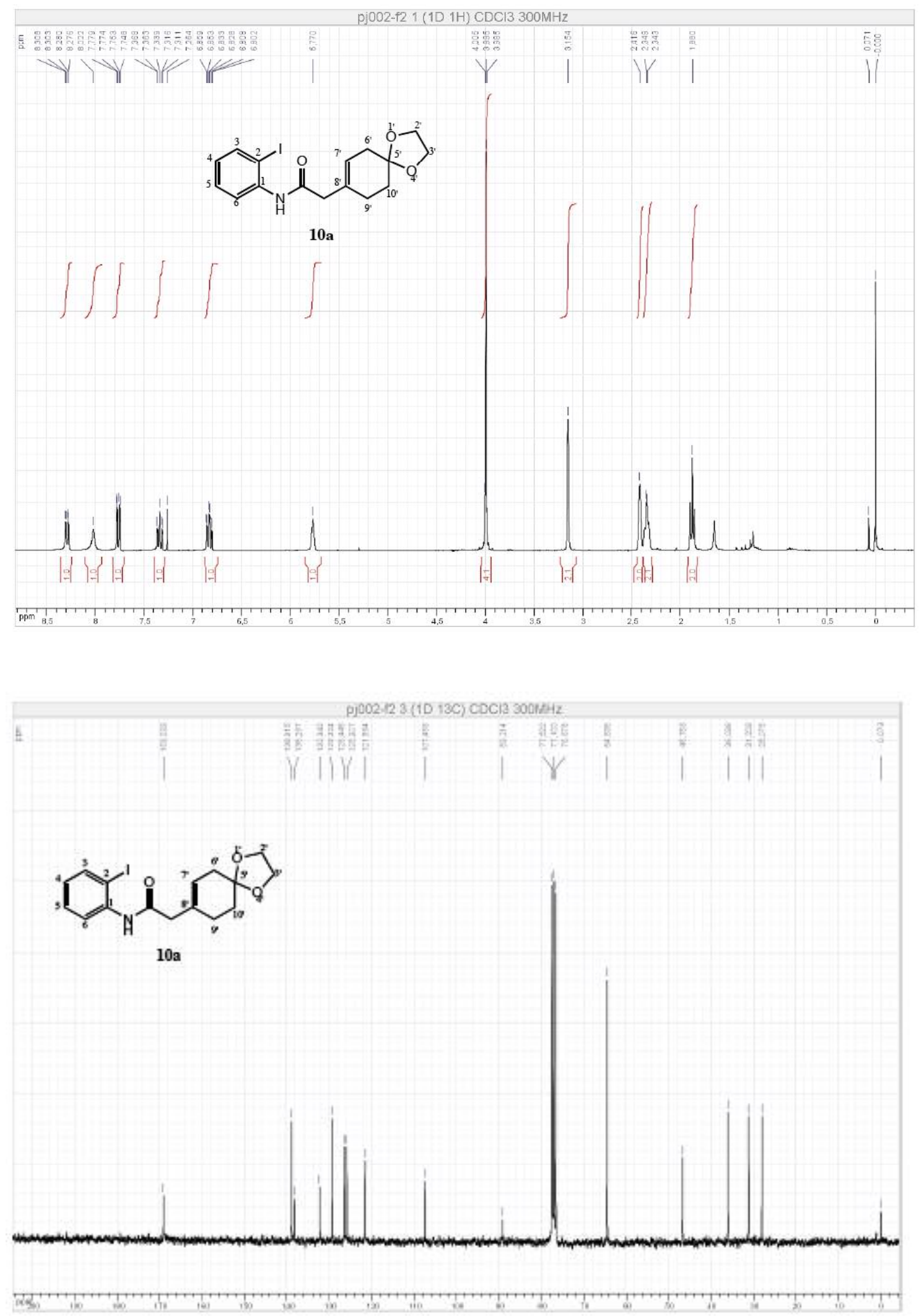

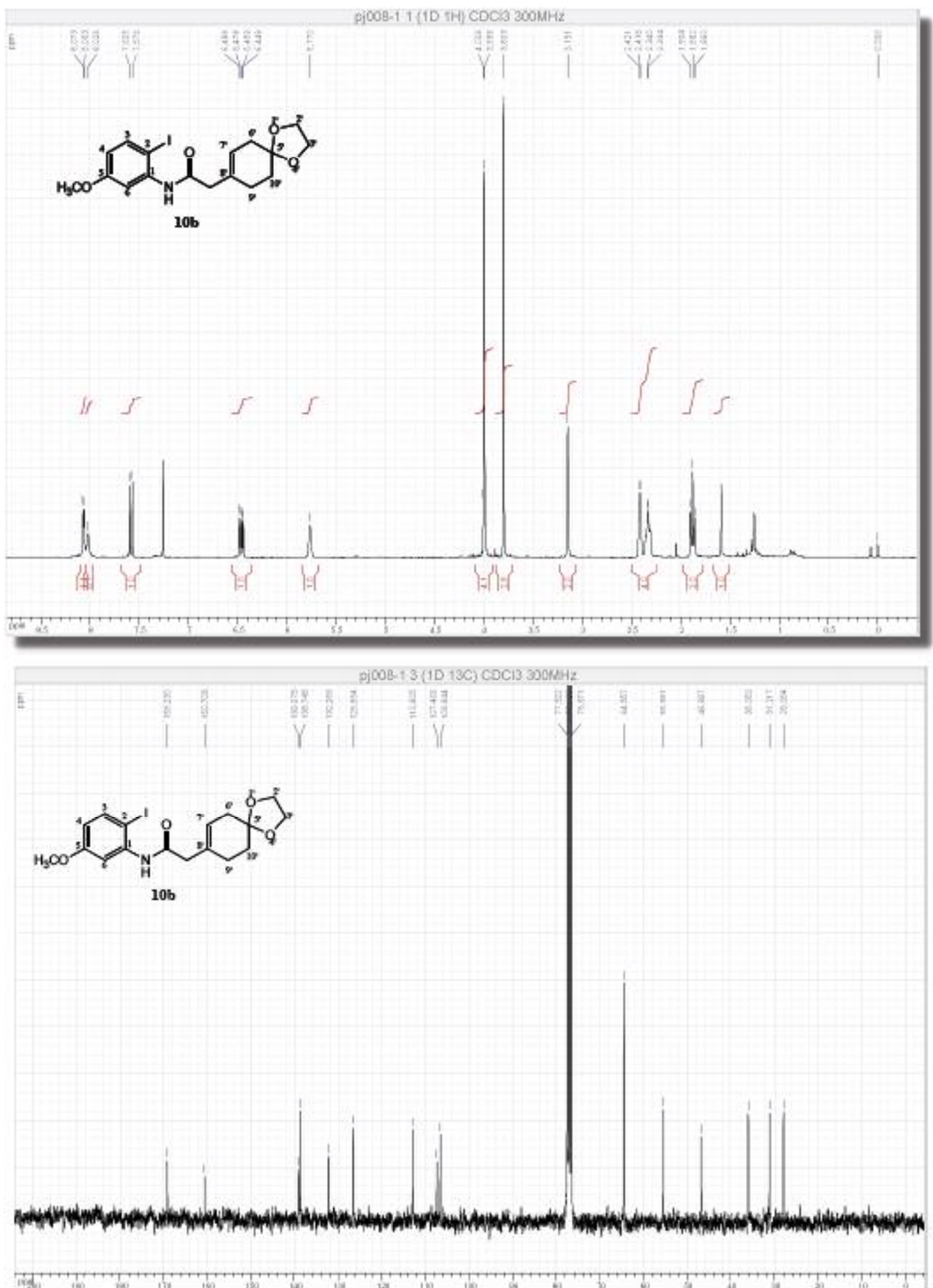

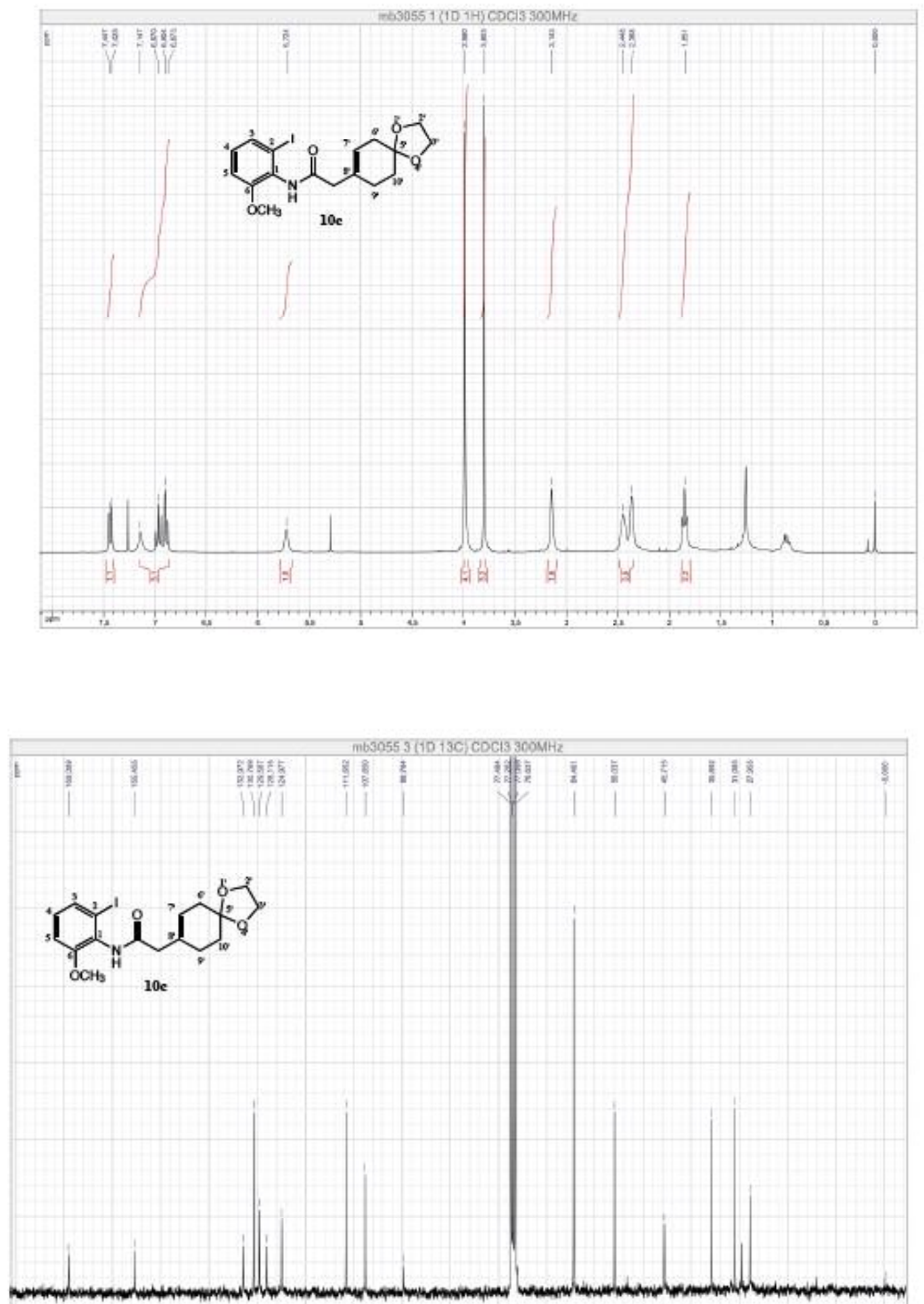

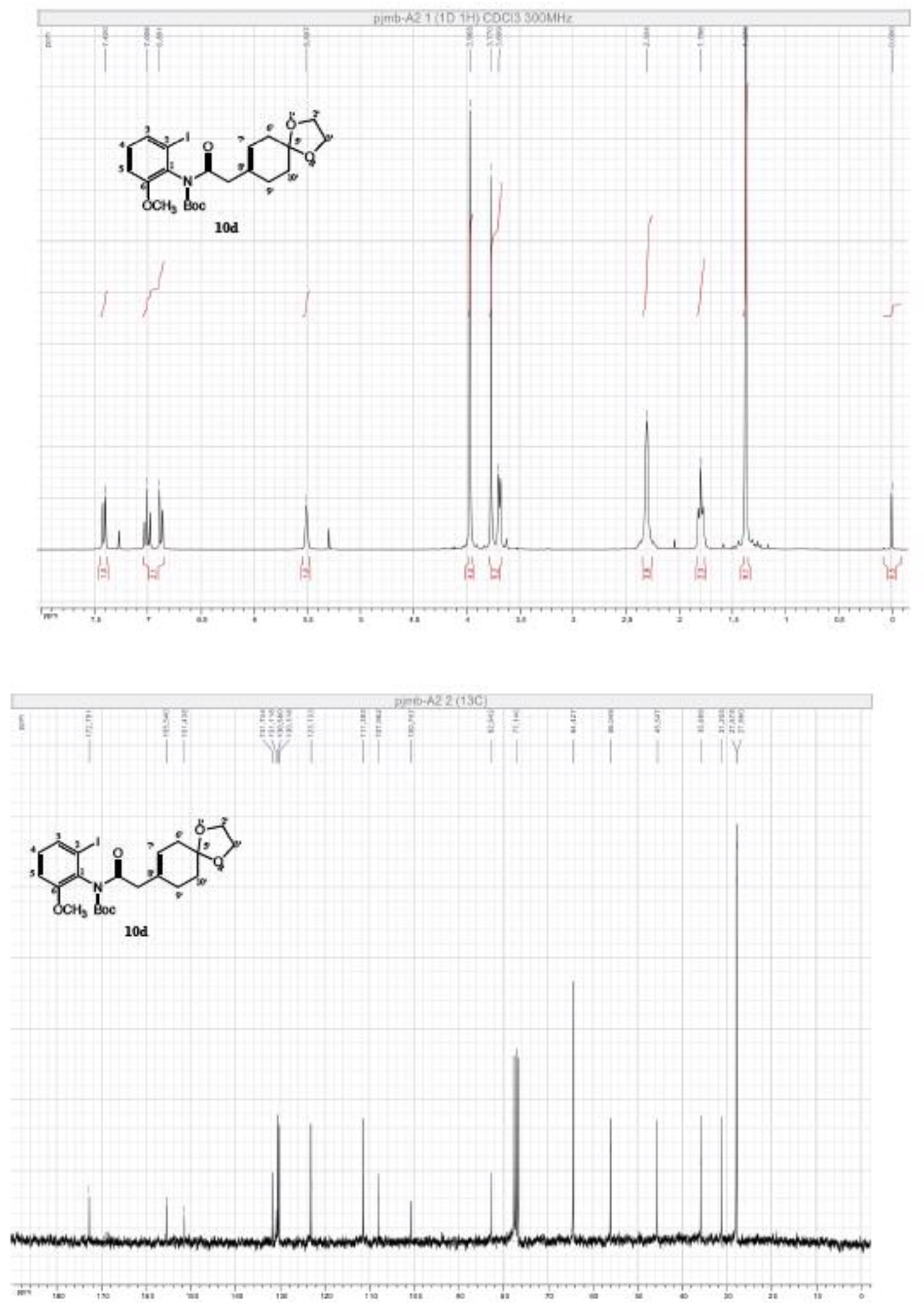

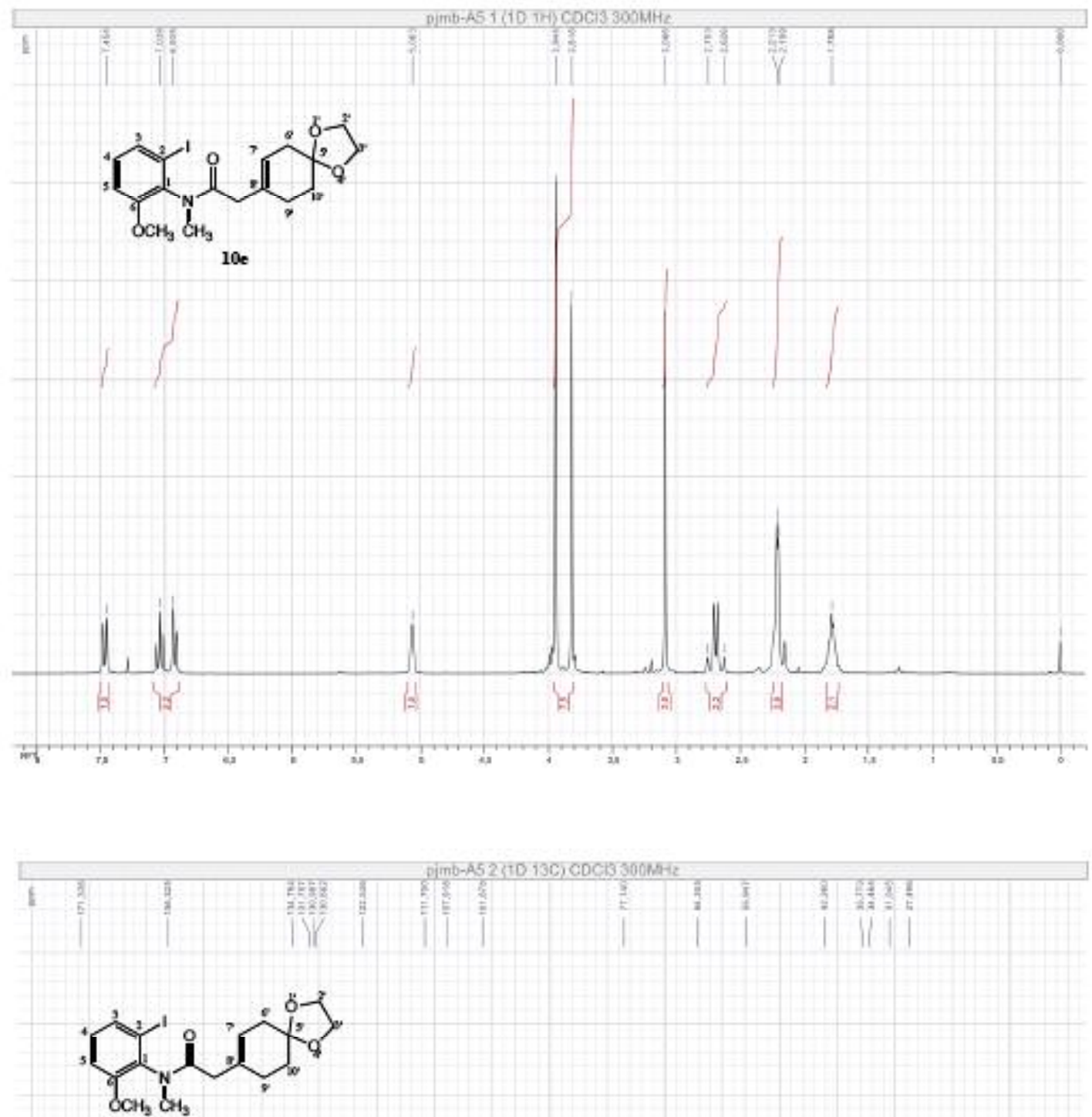

10 e
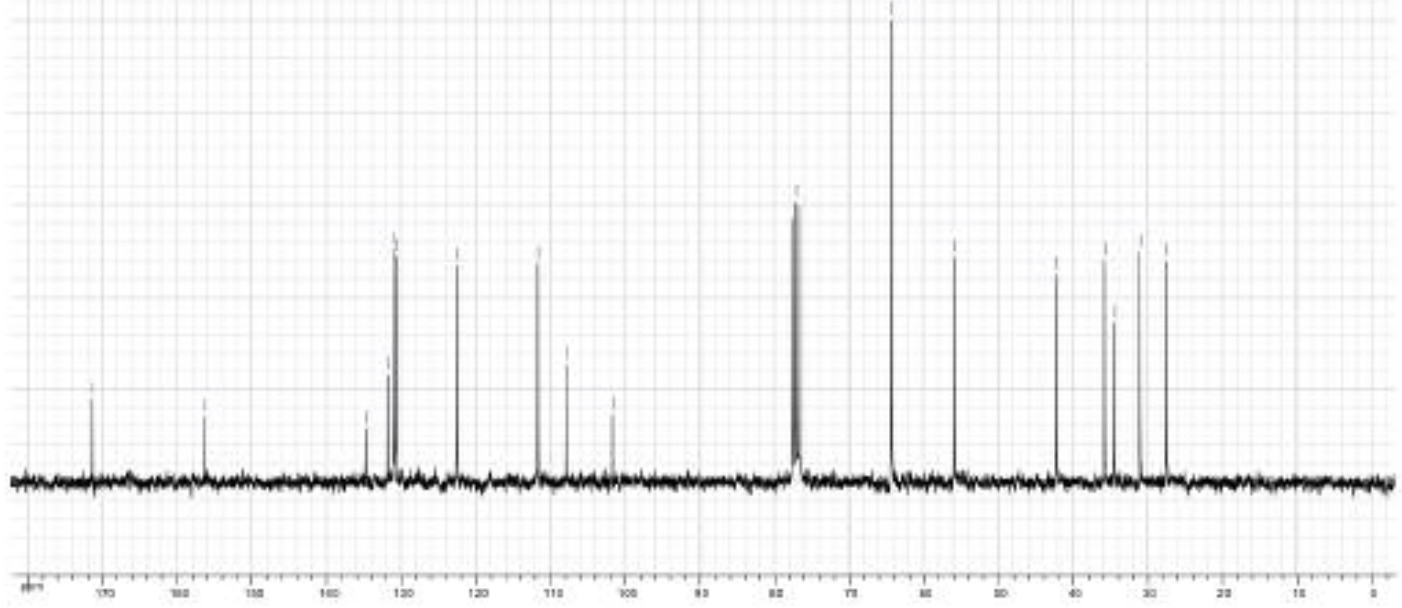

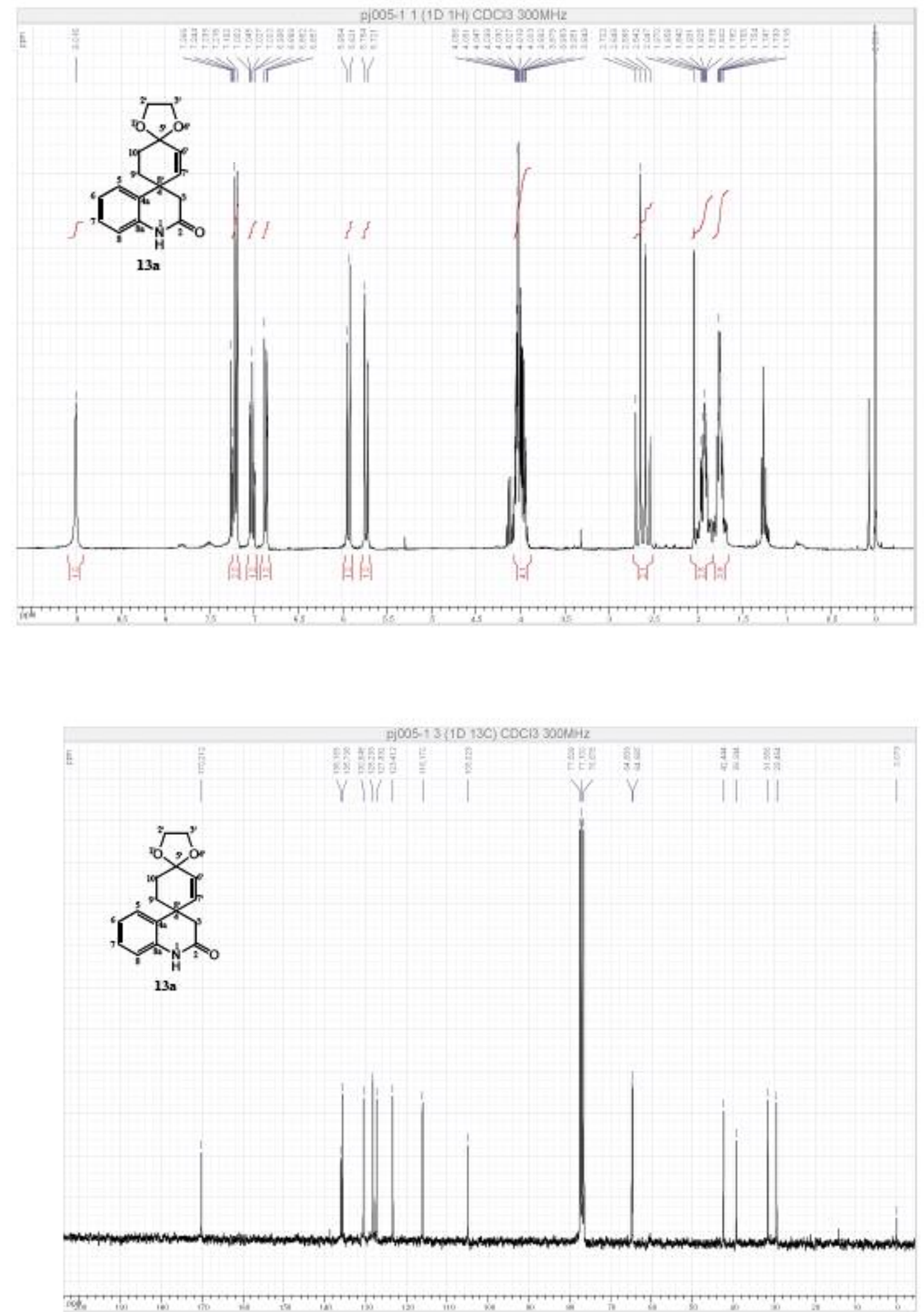

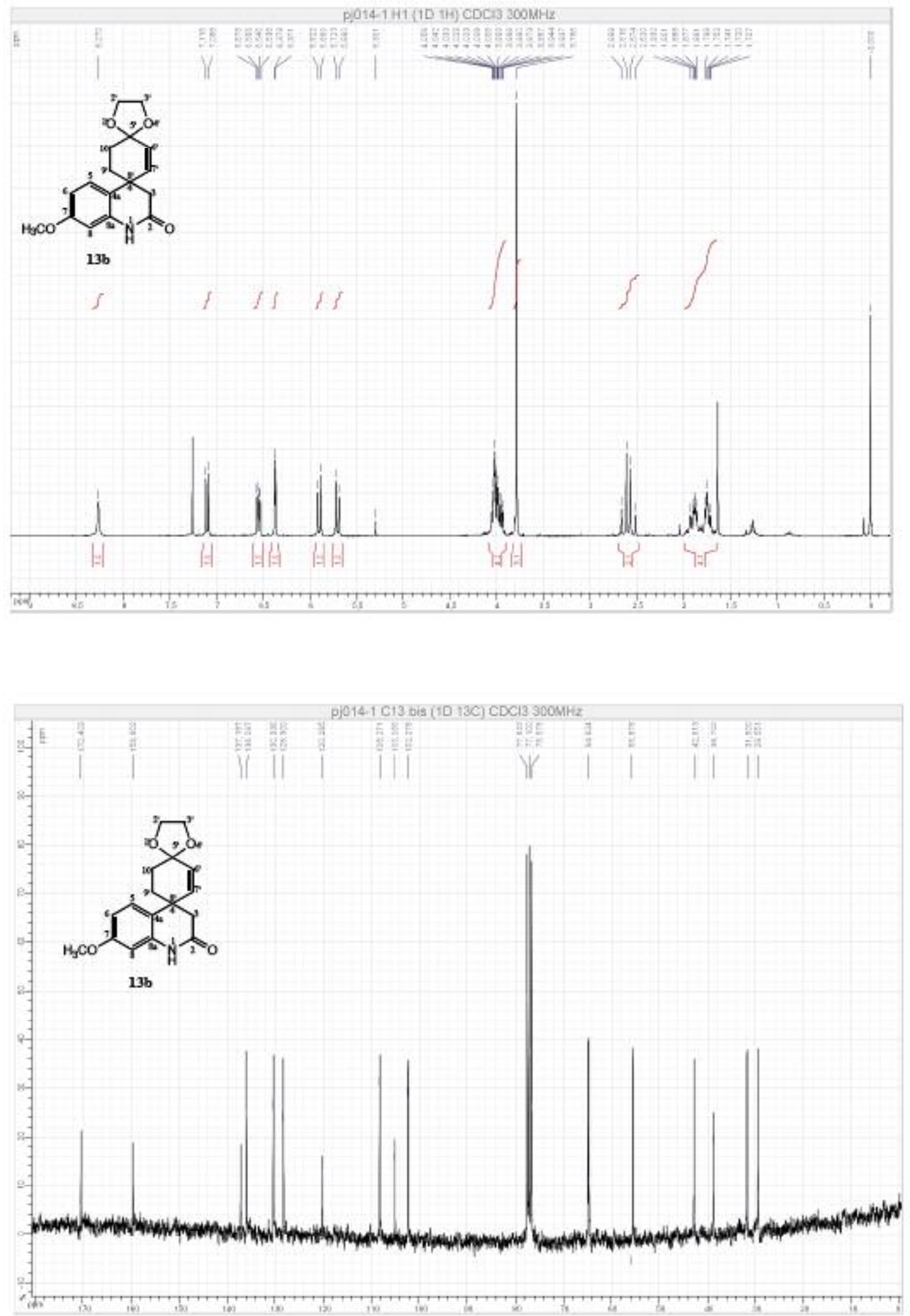

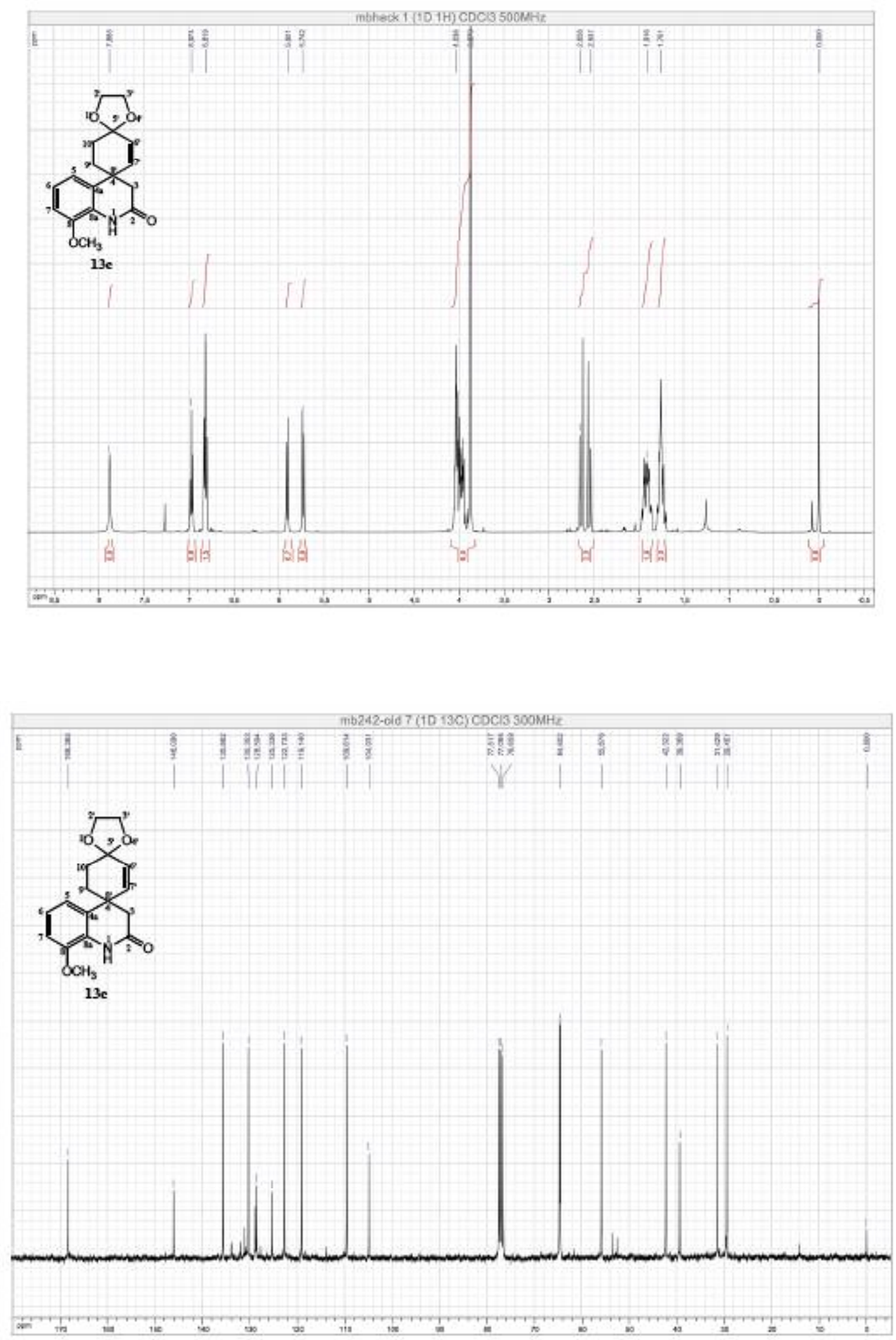

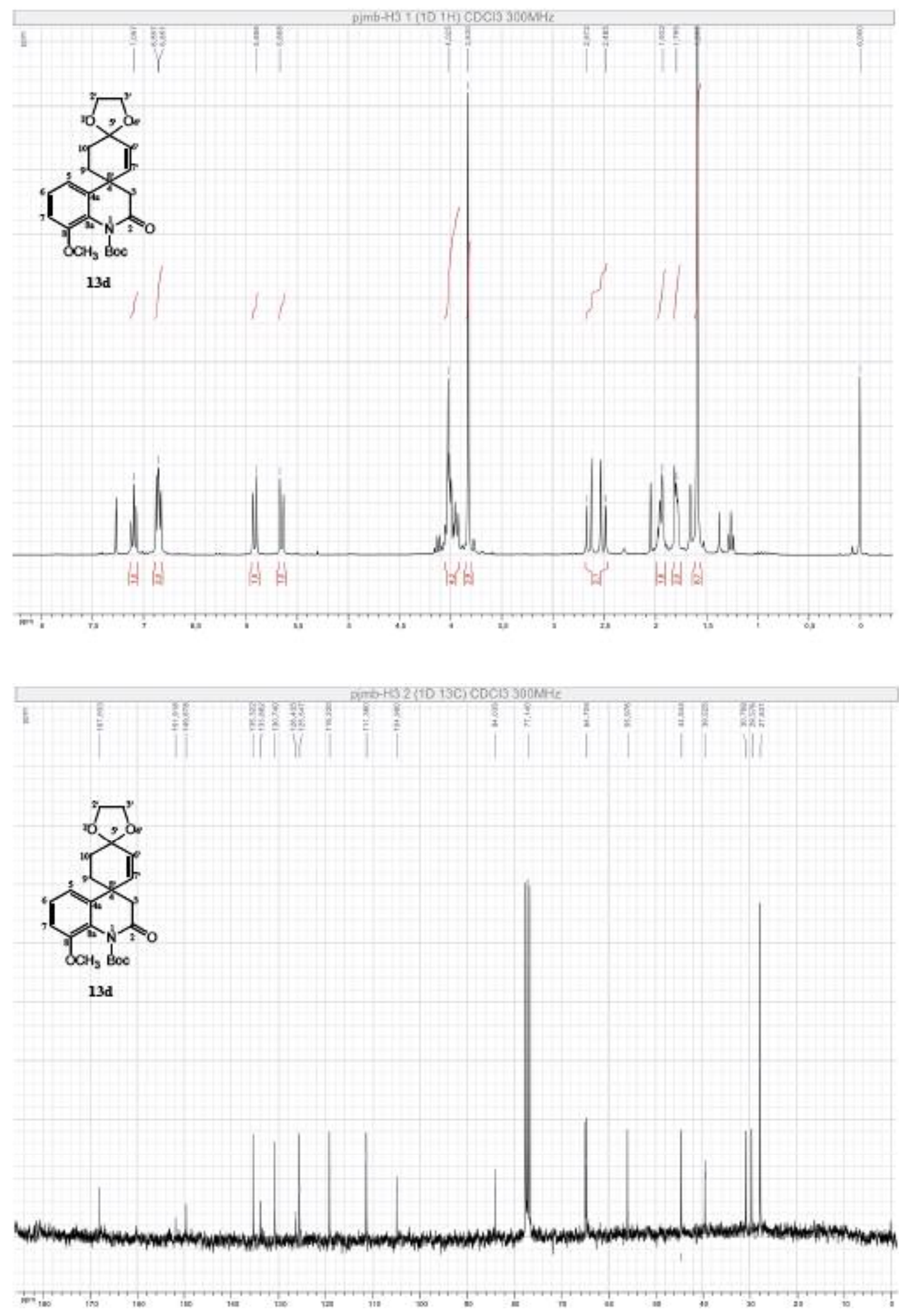

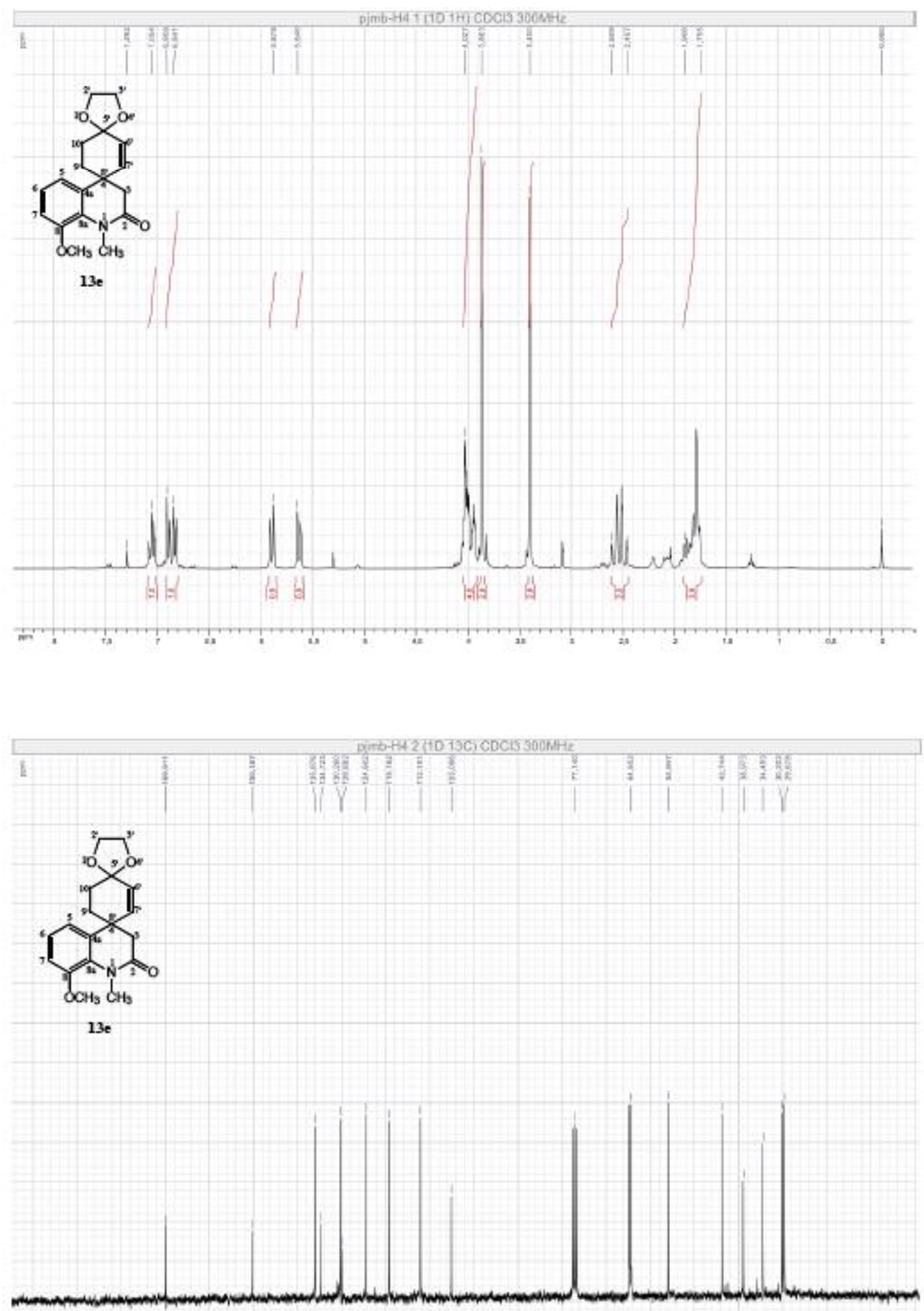

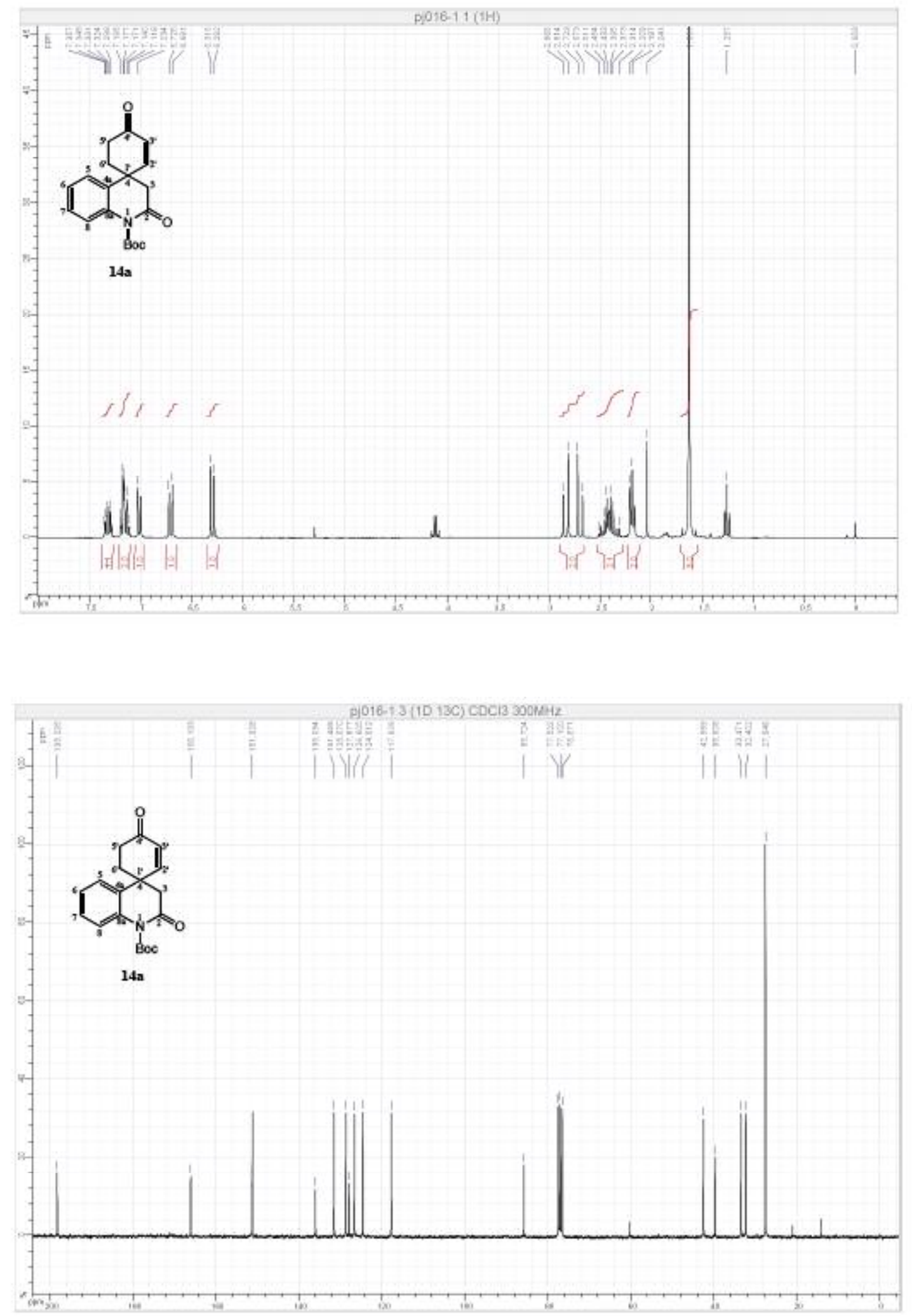

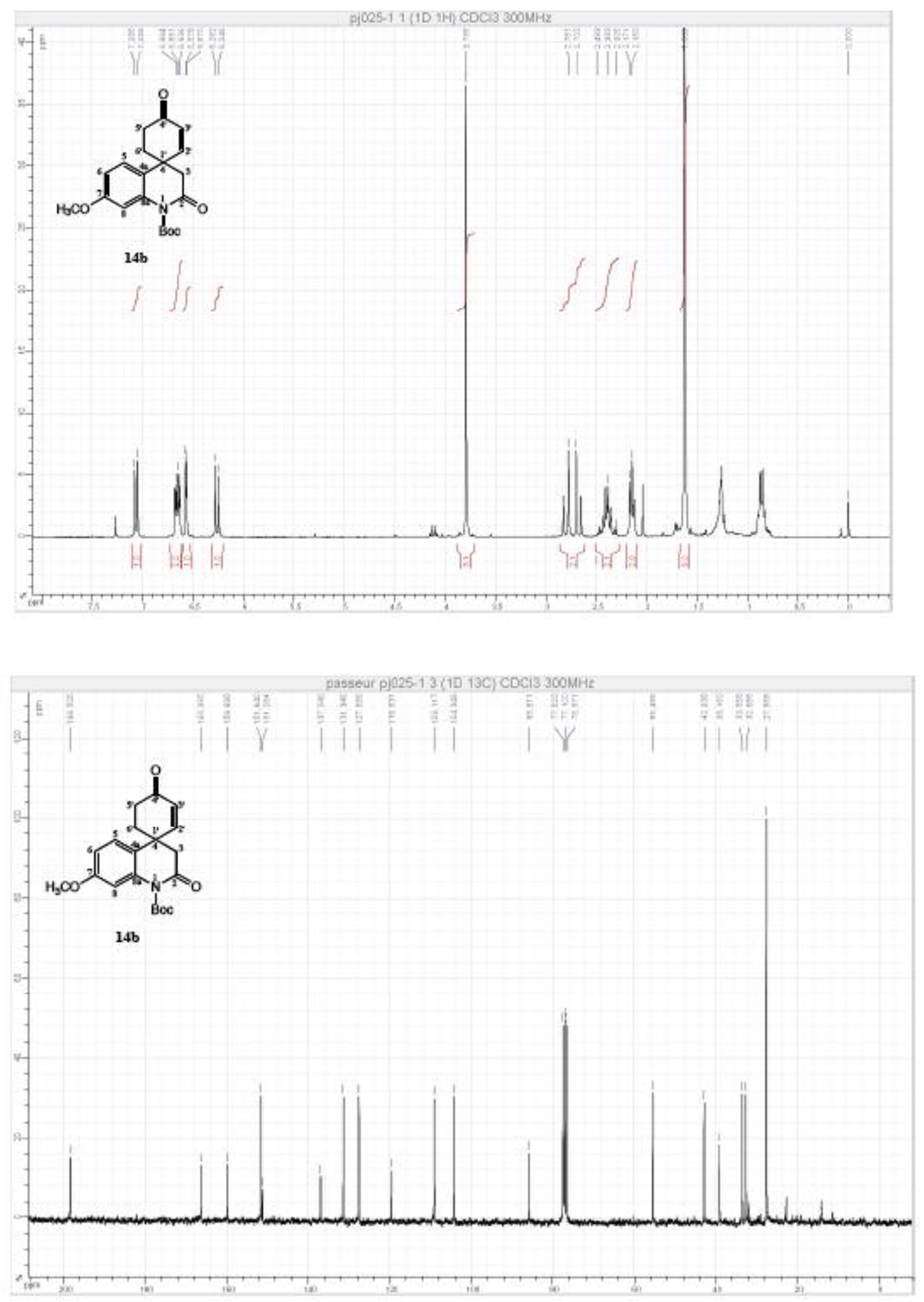

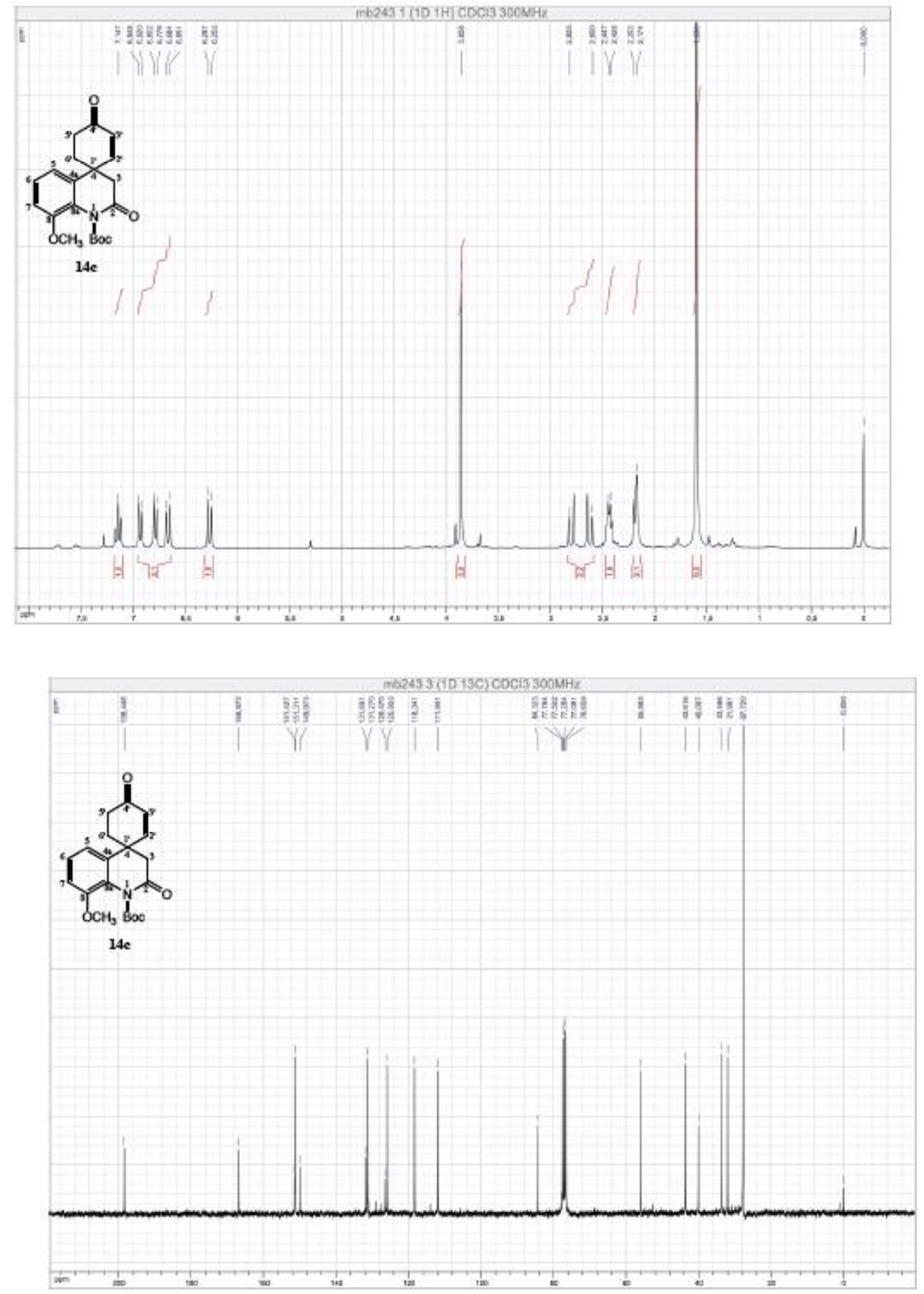

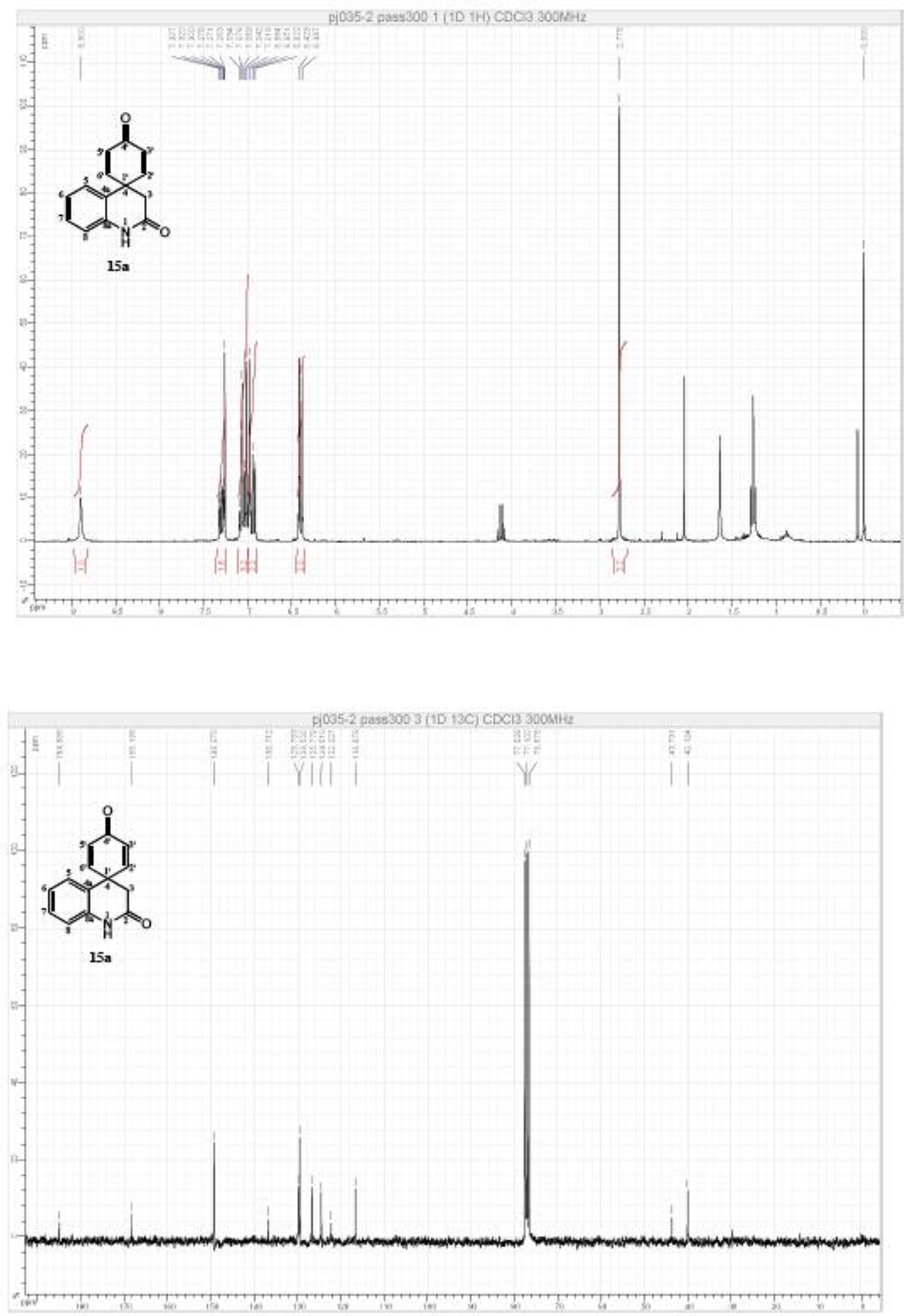

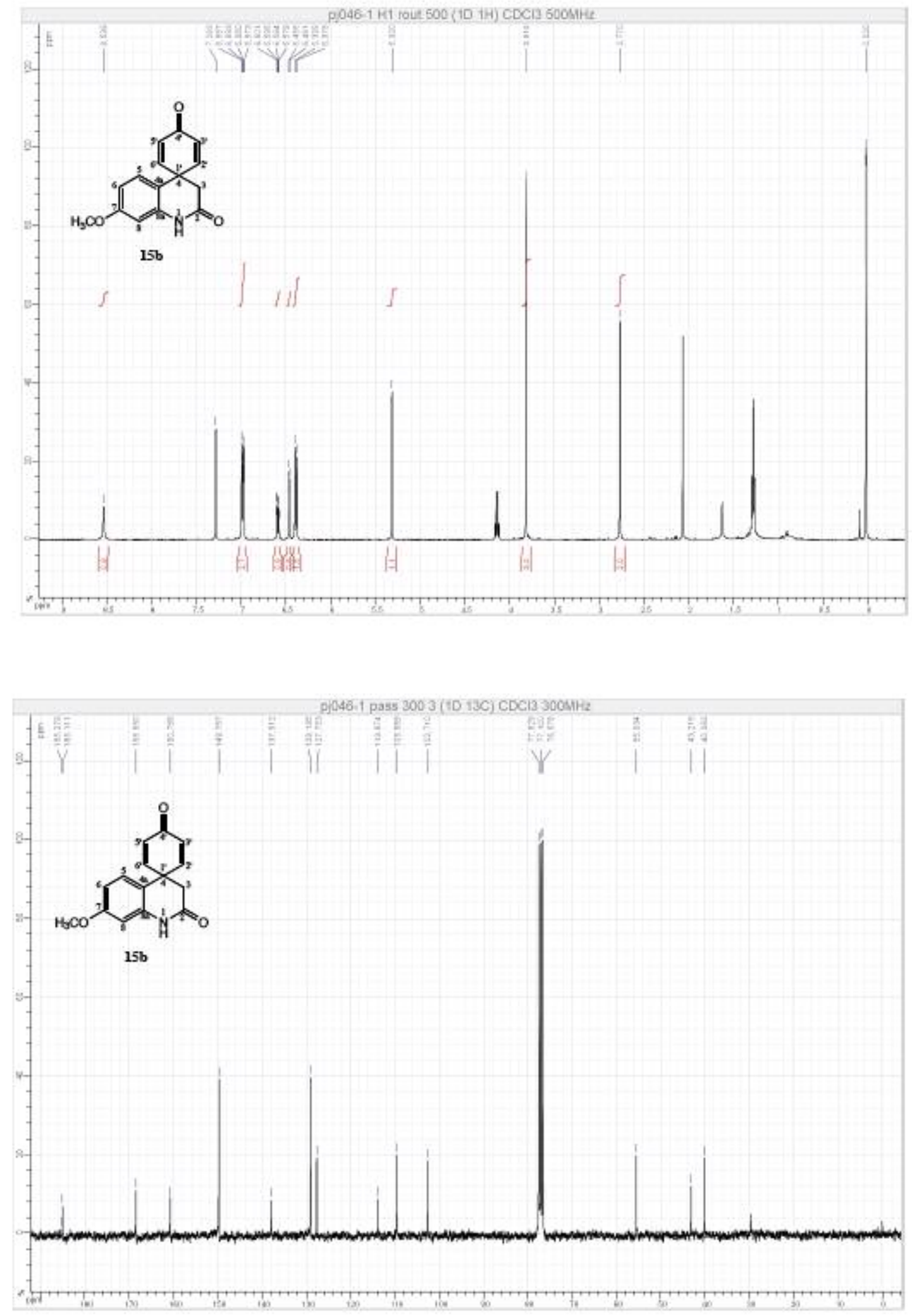

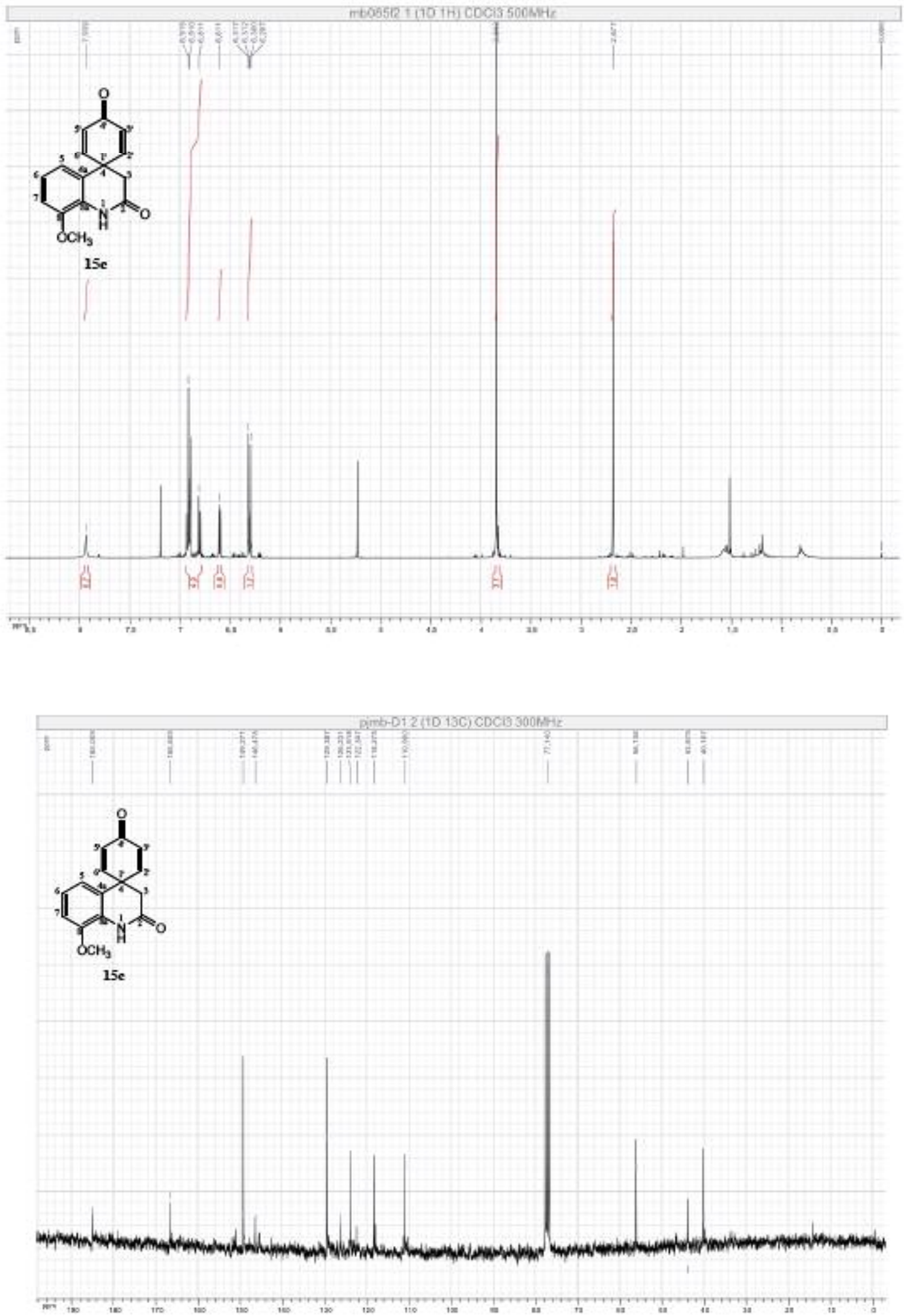

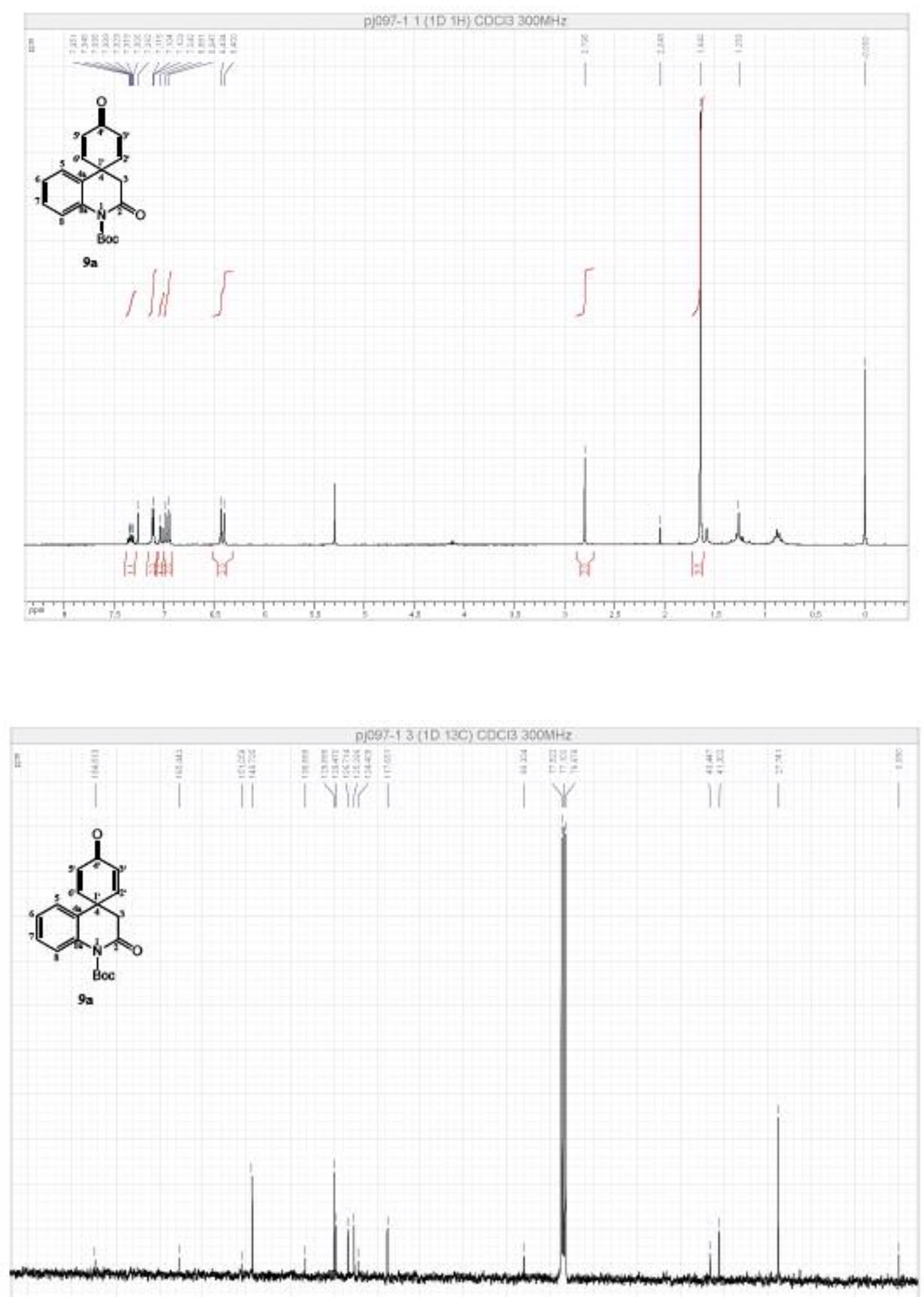

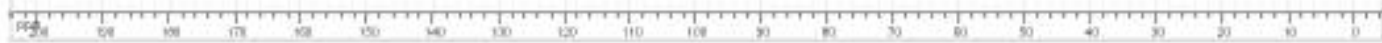



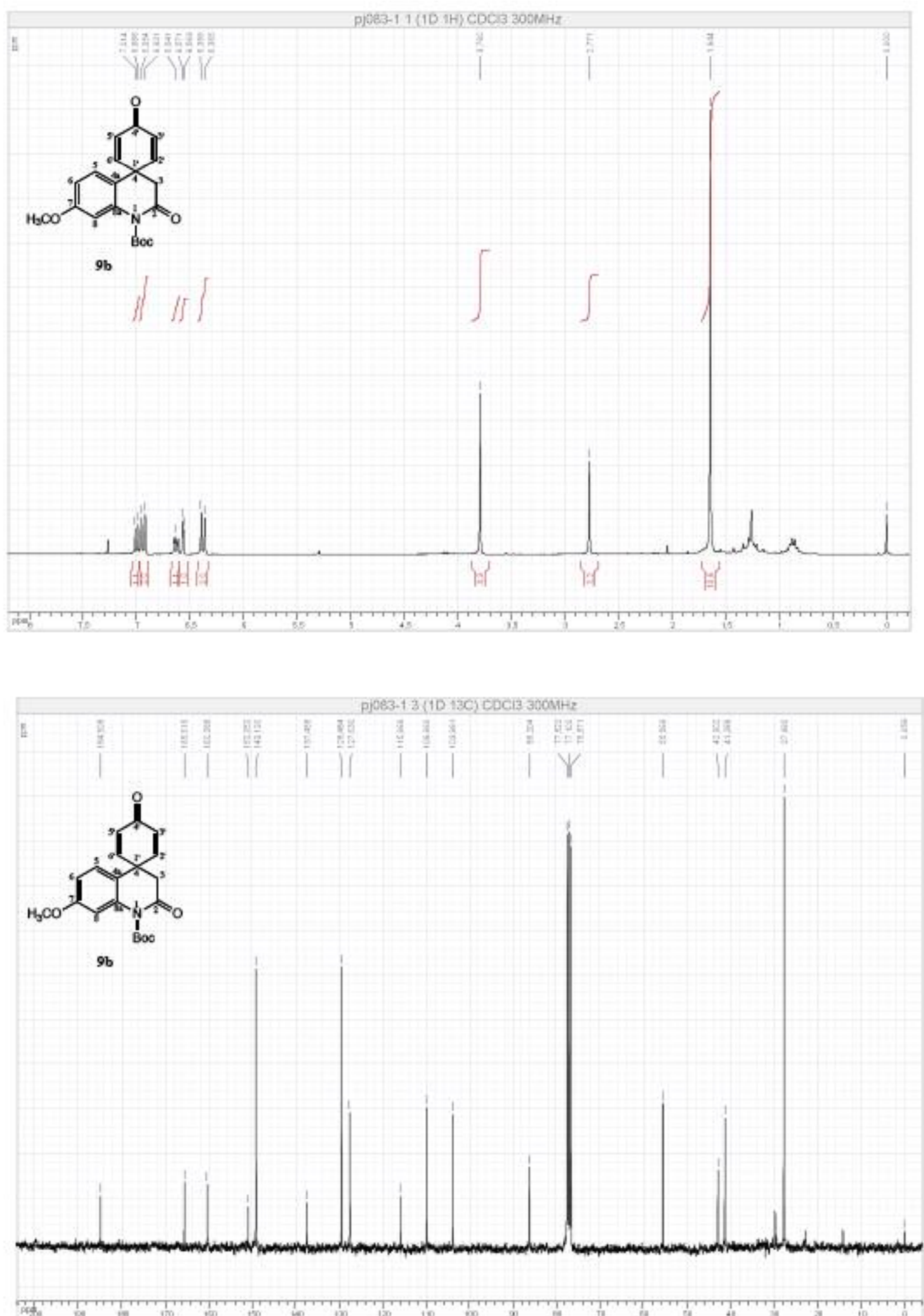

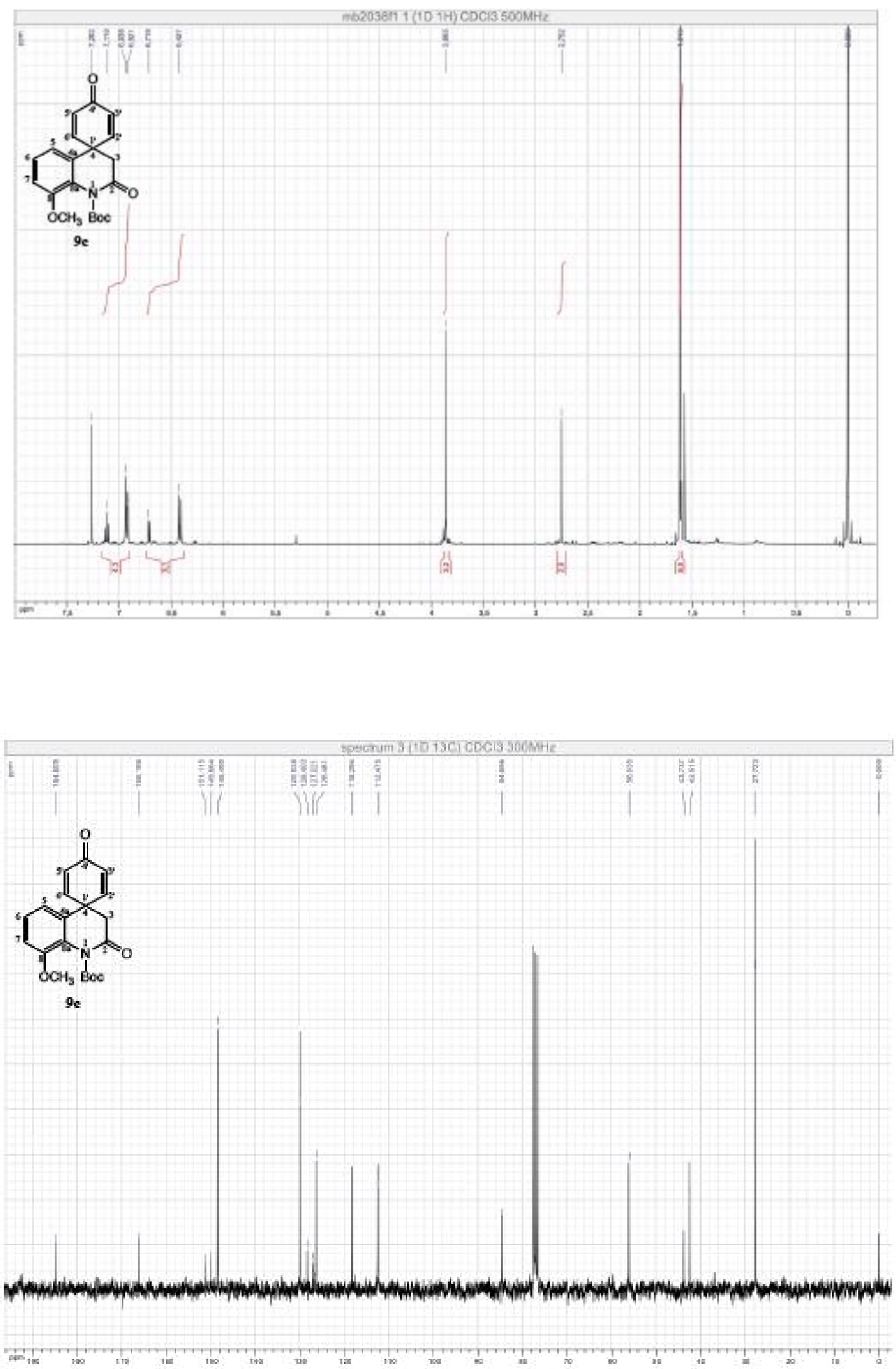

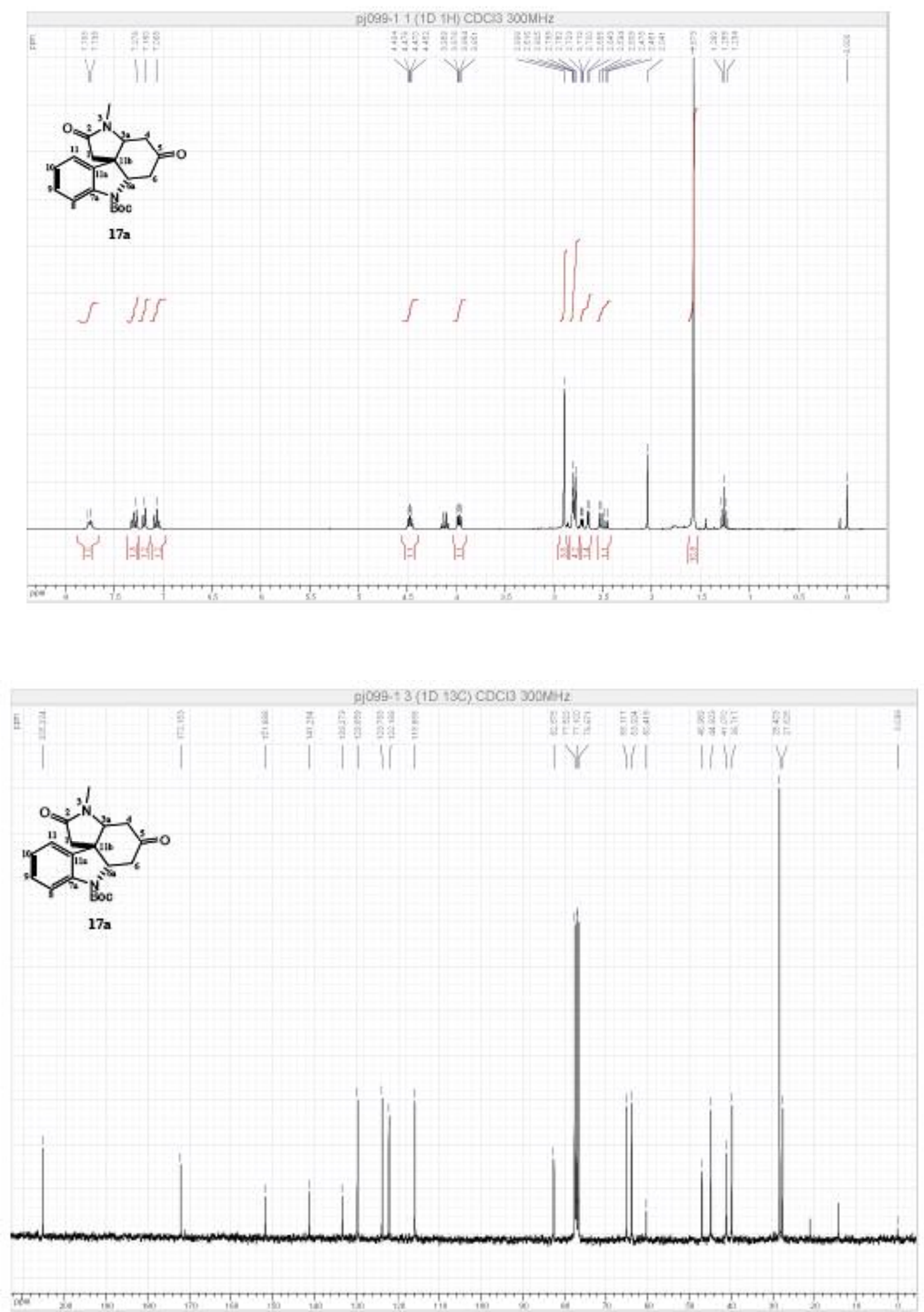

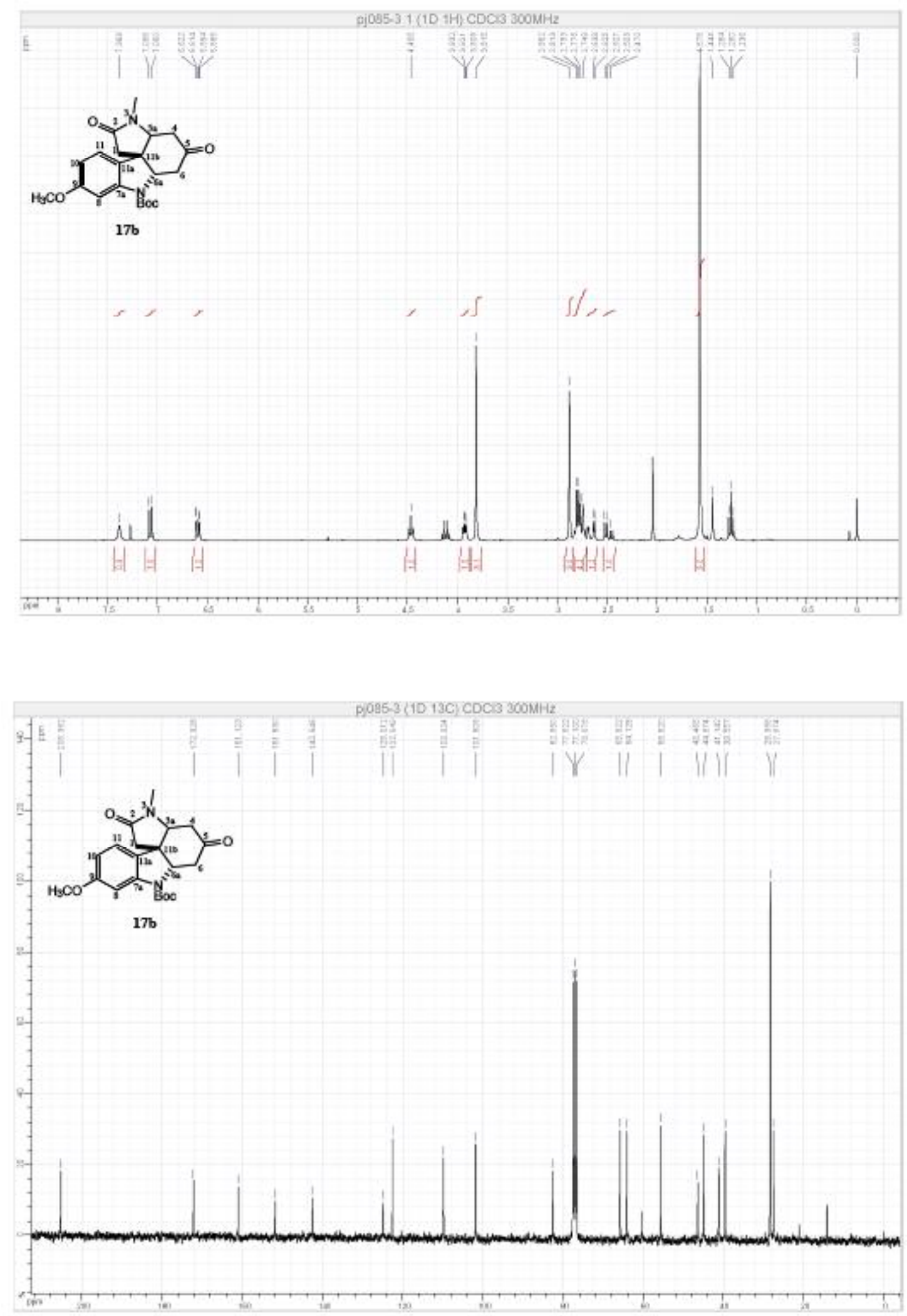

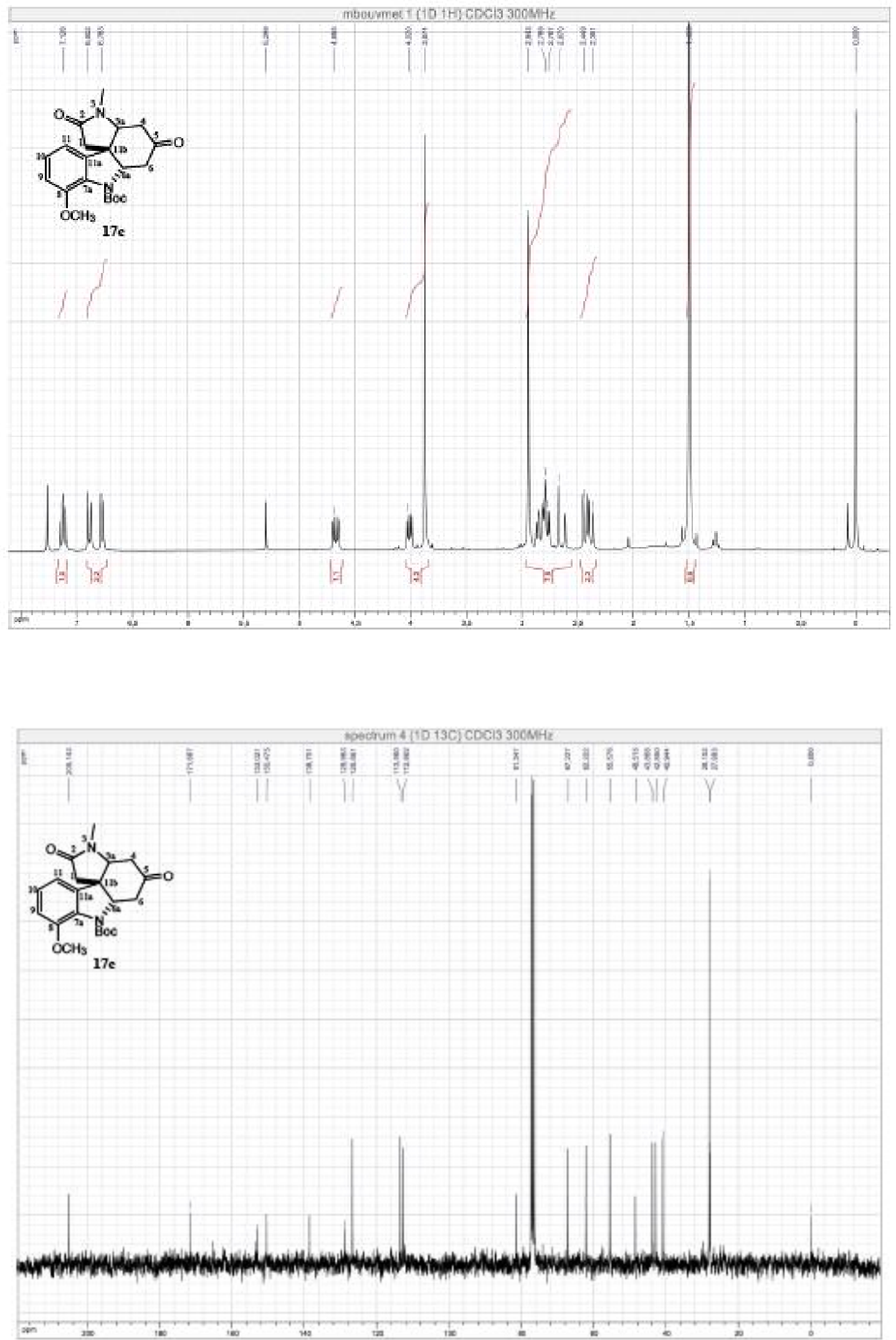

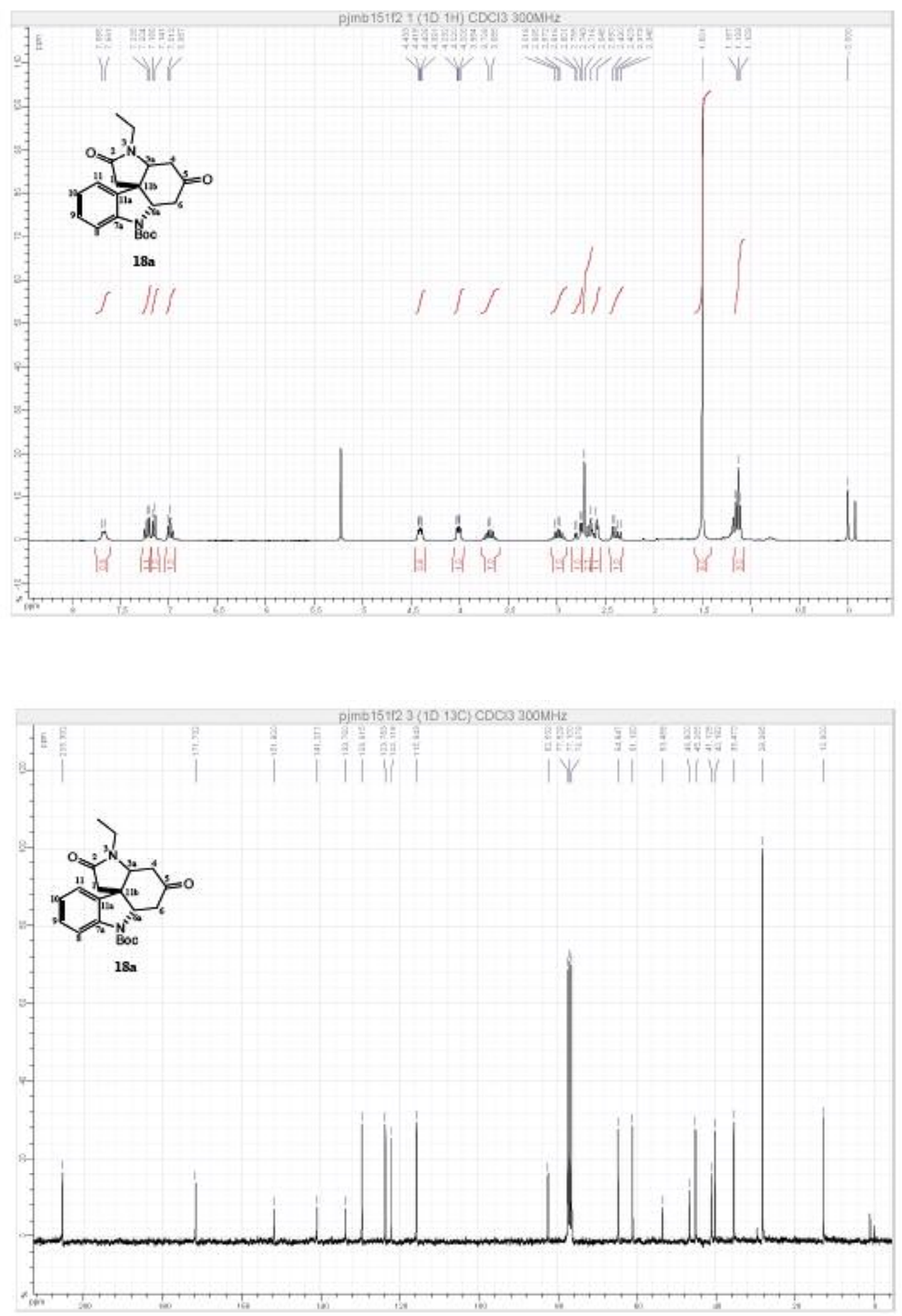

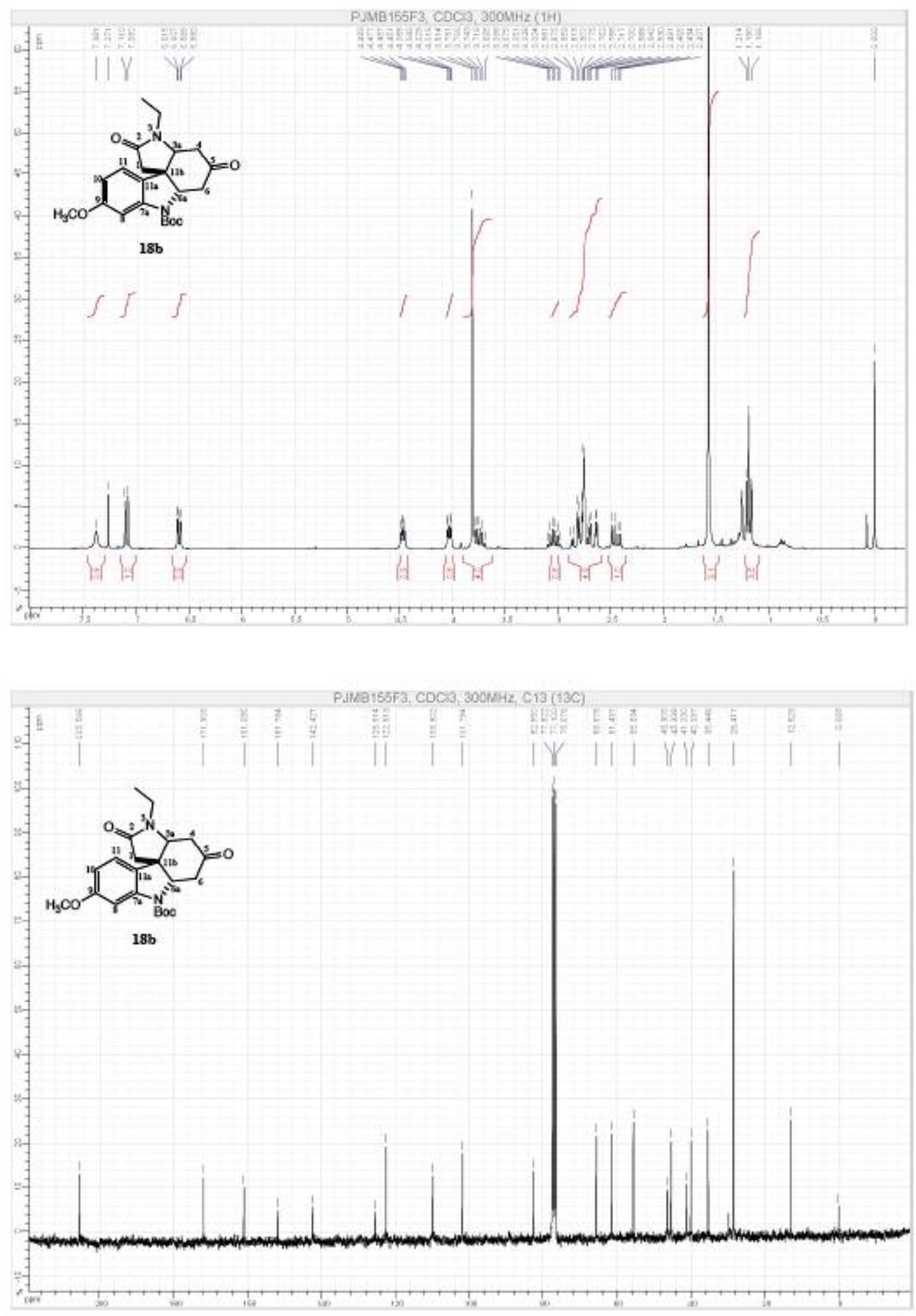

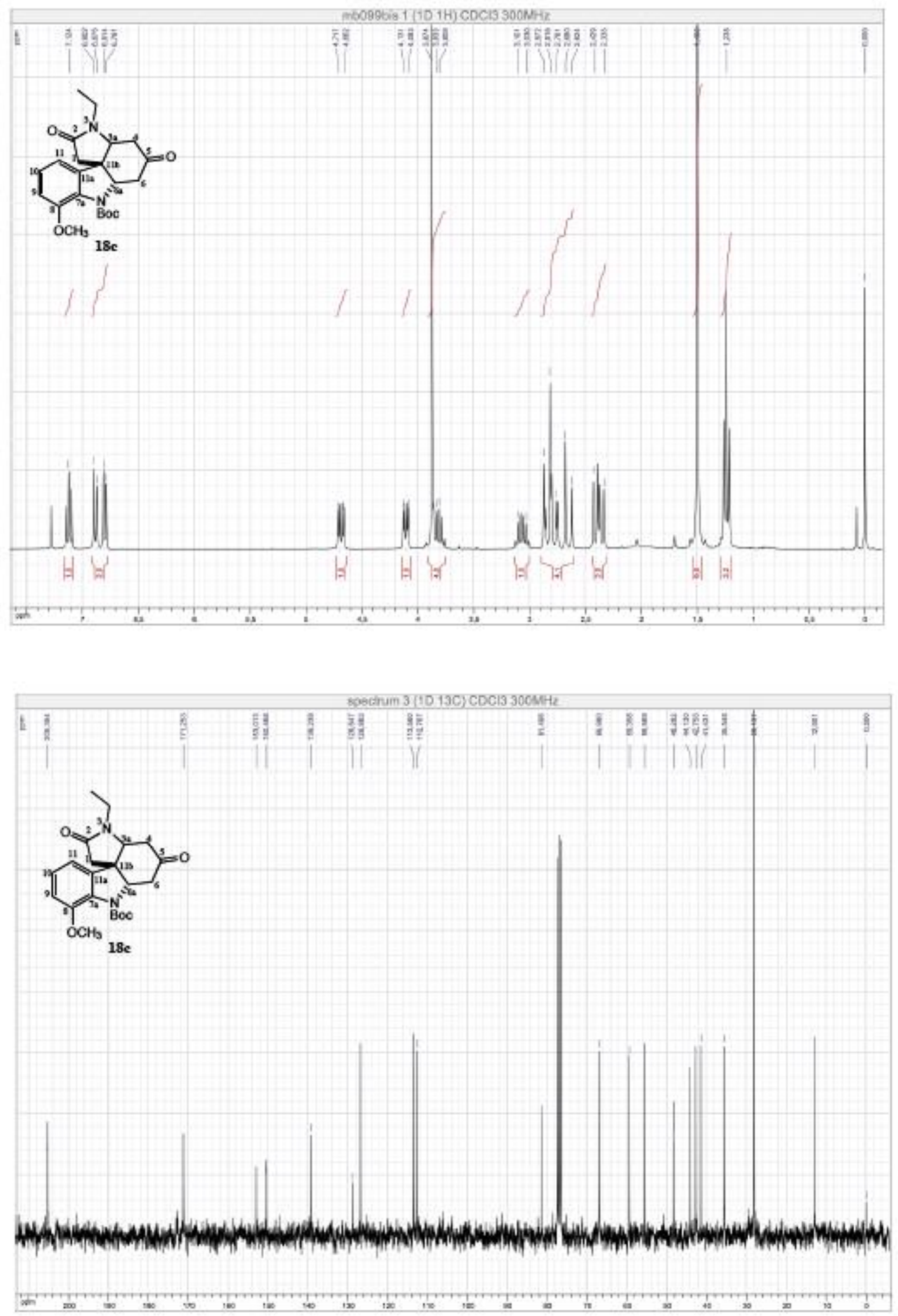

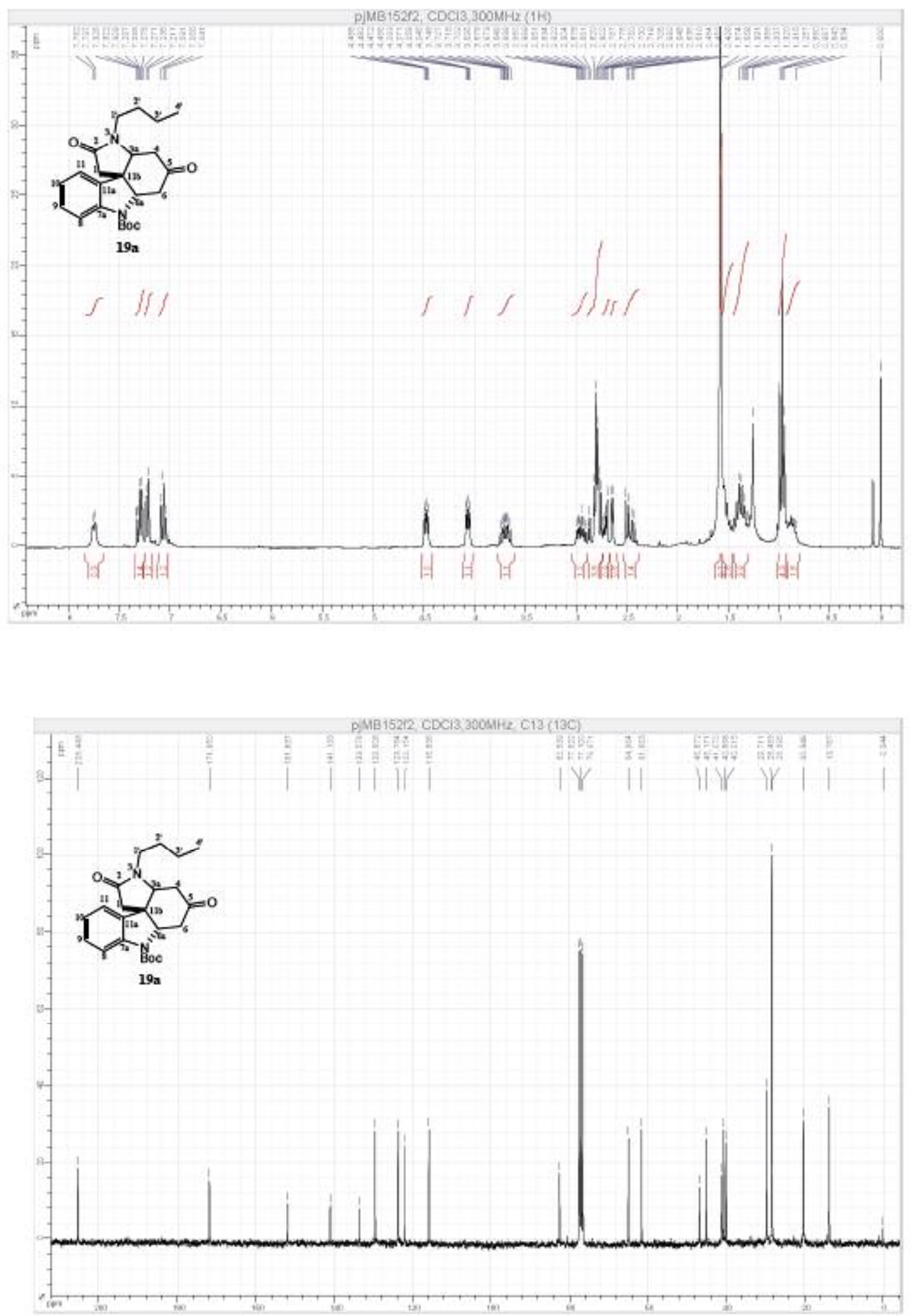

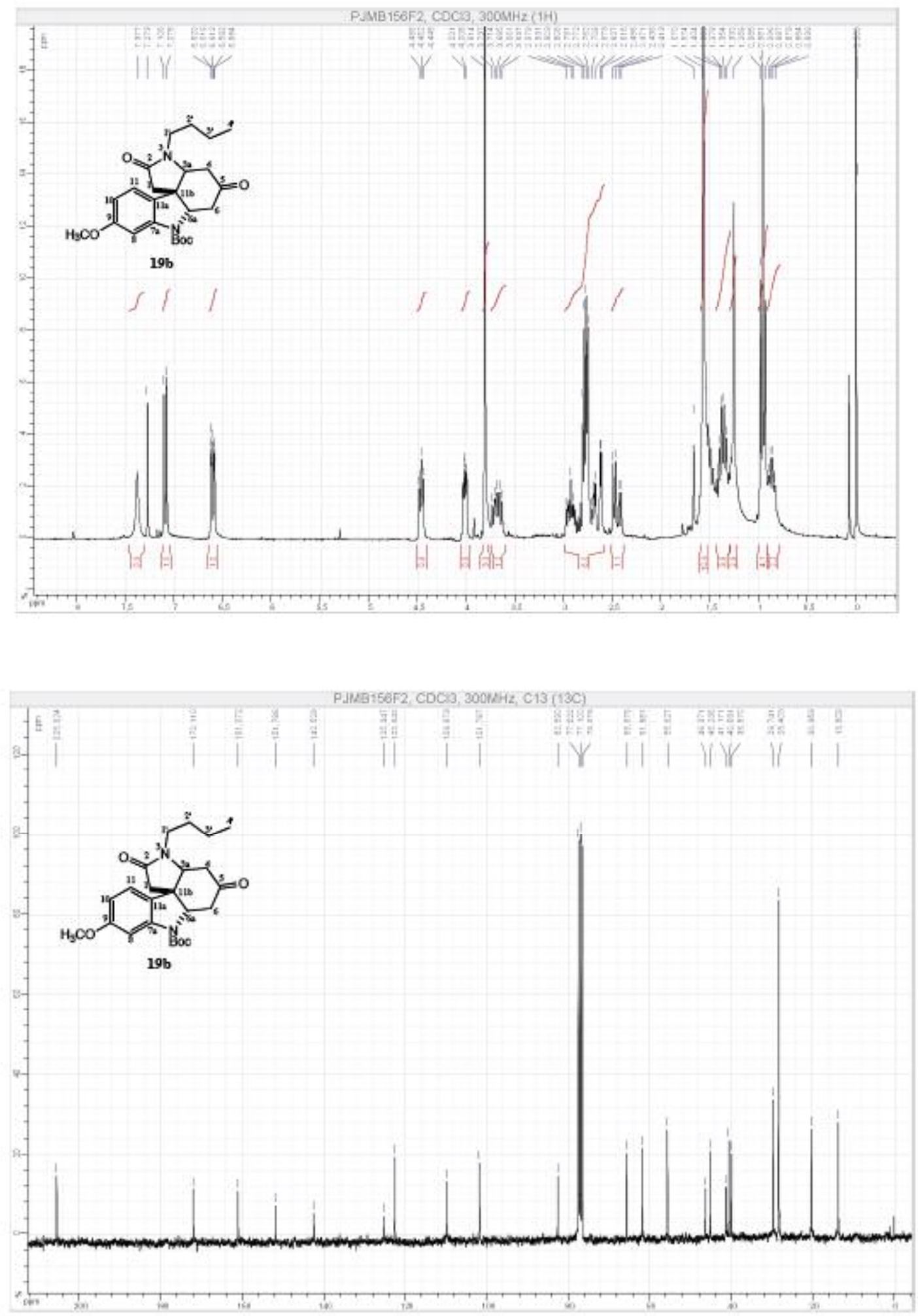

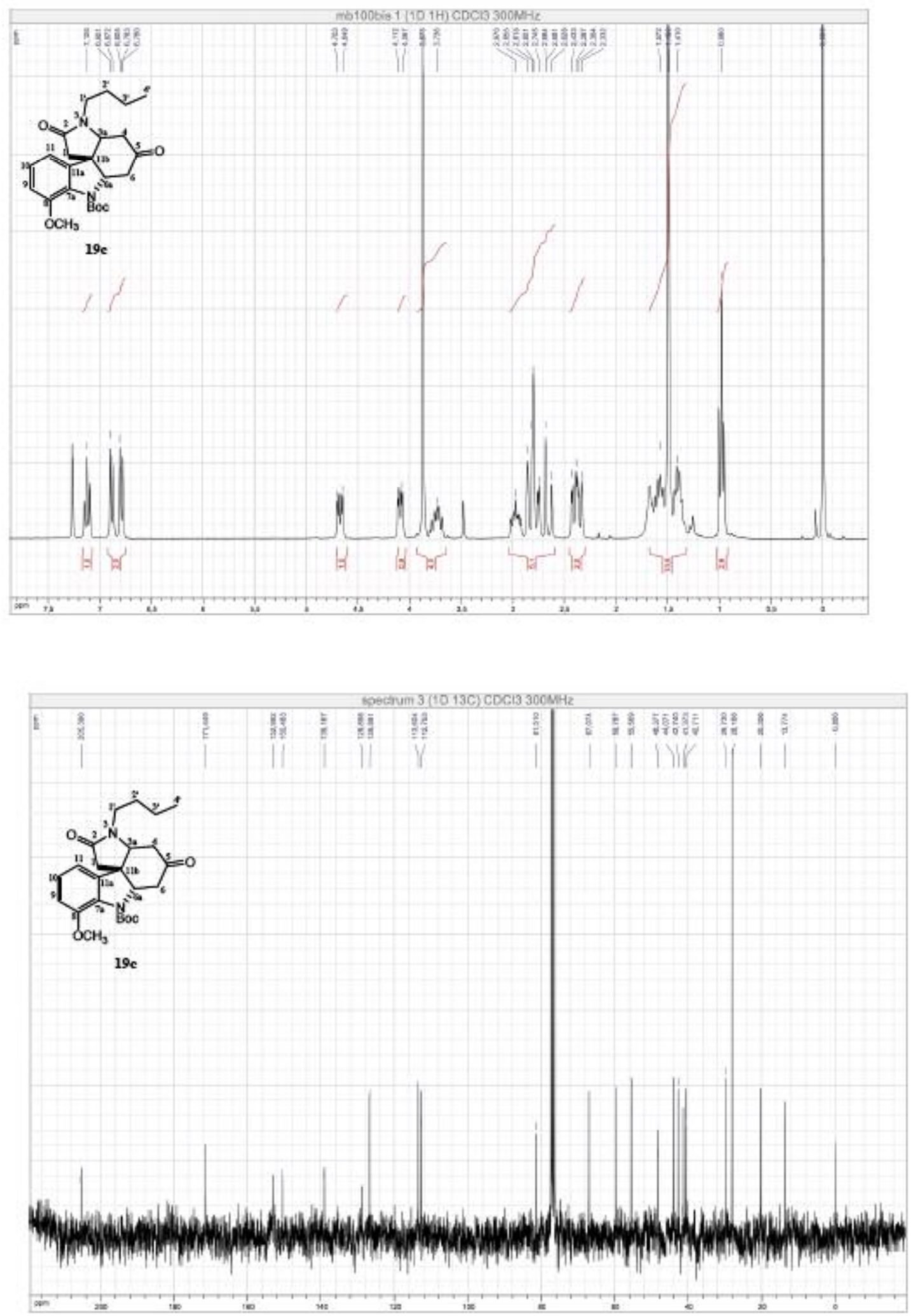

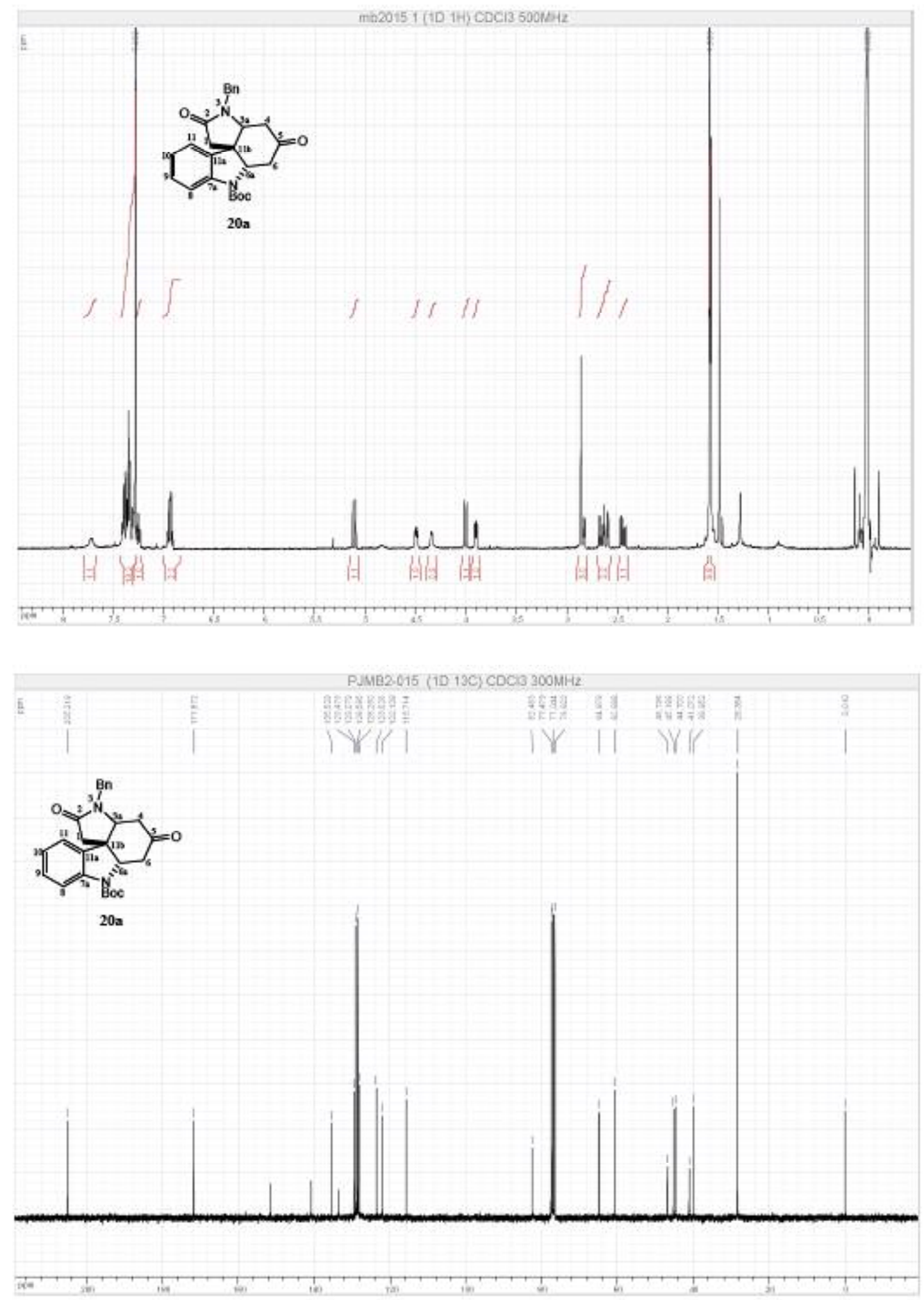

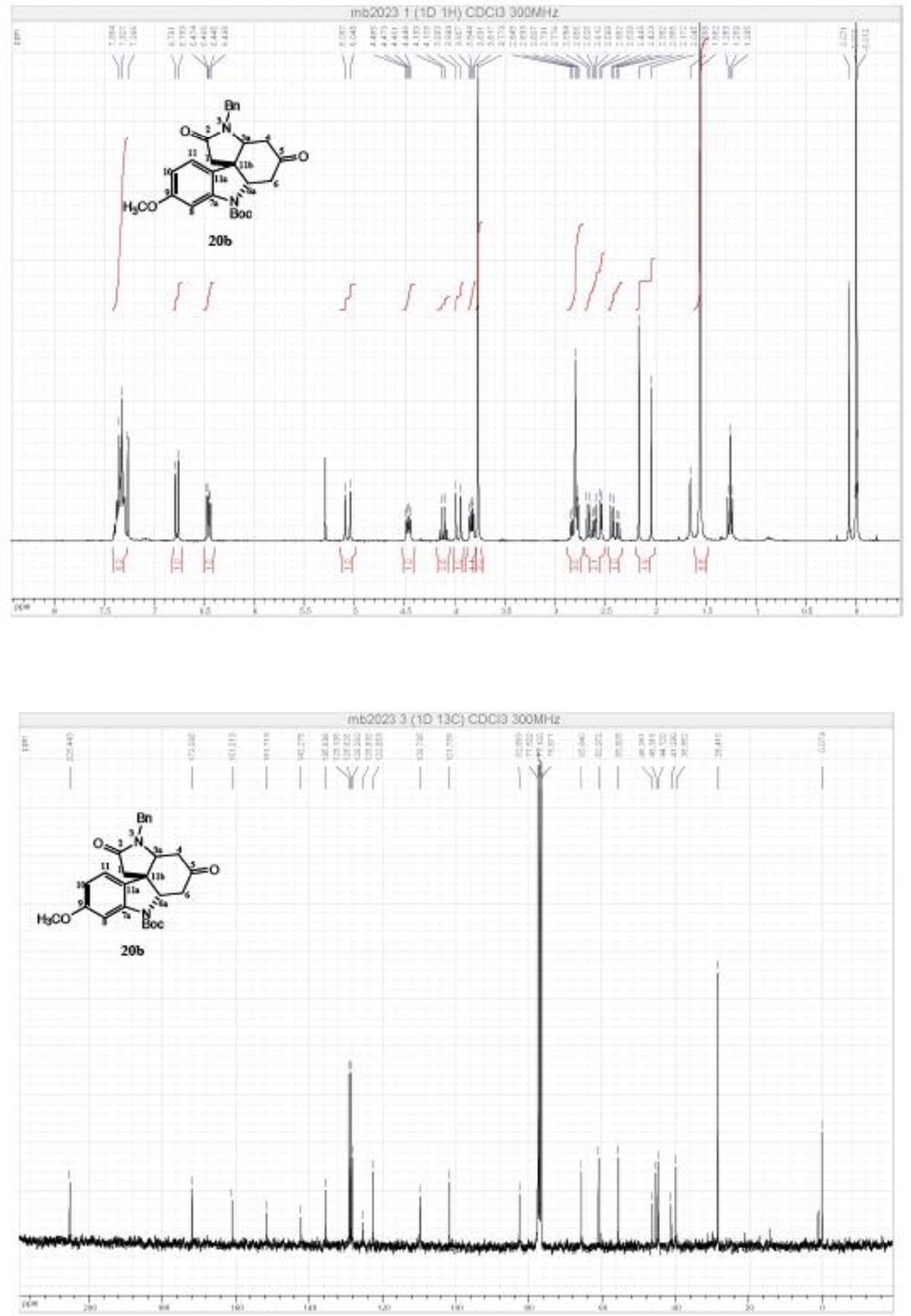

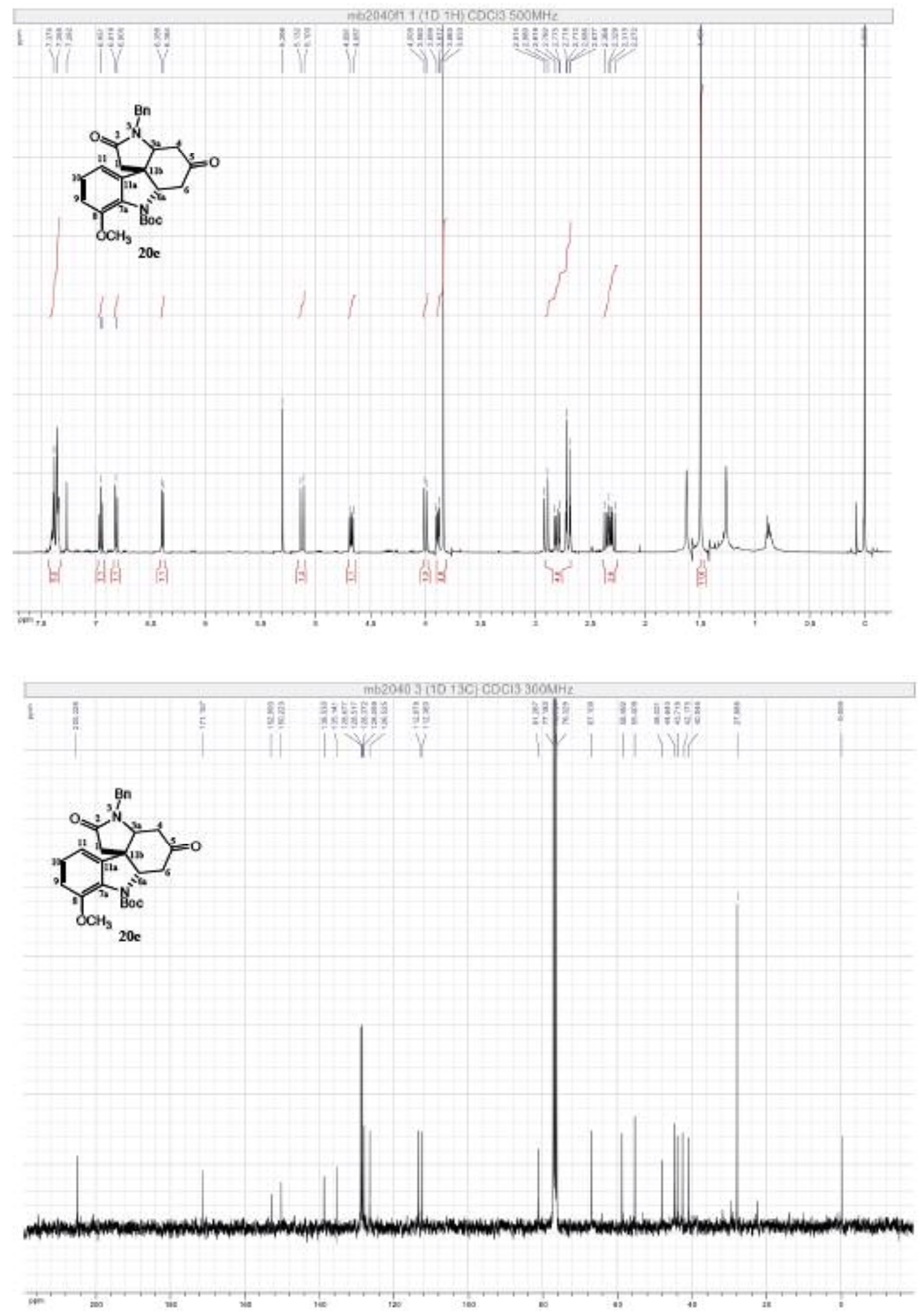

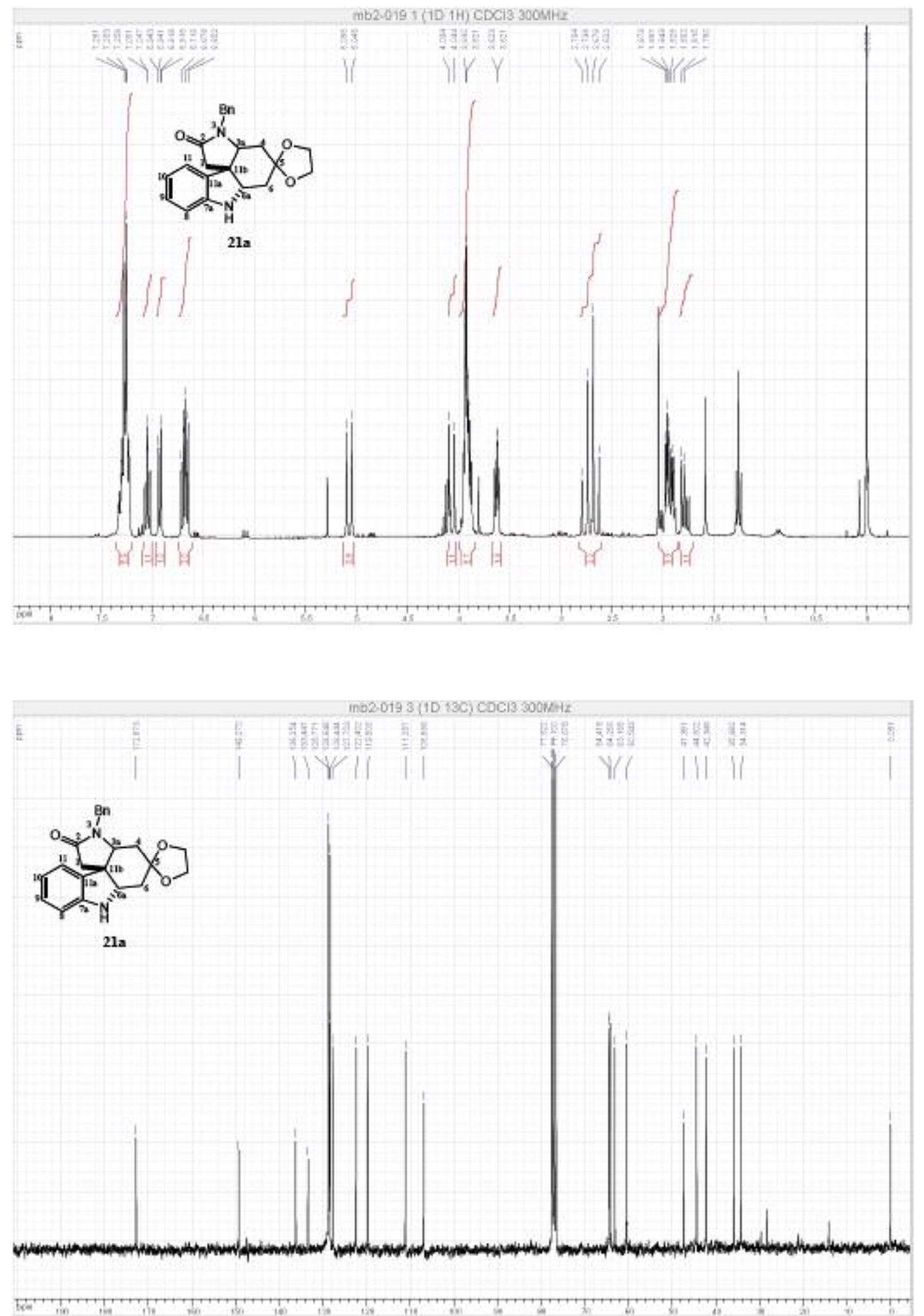

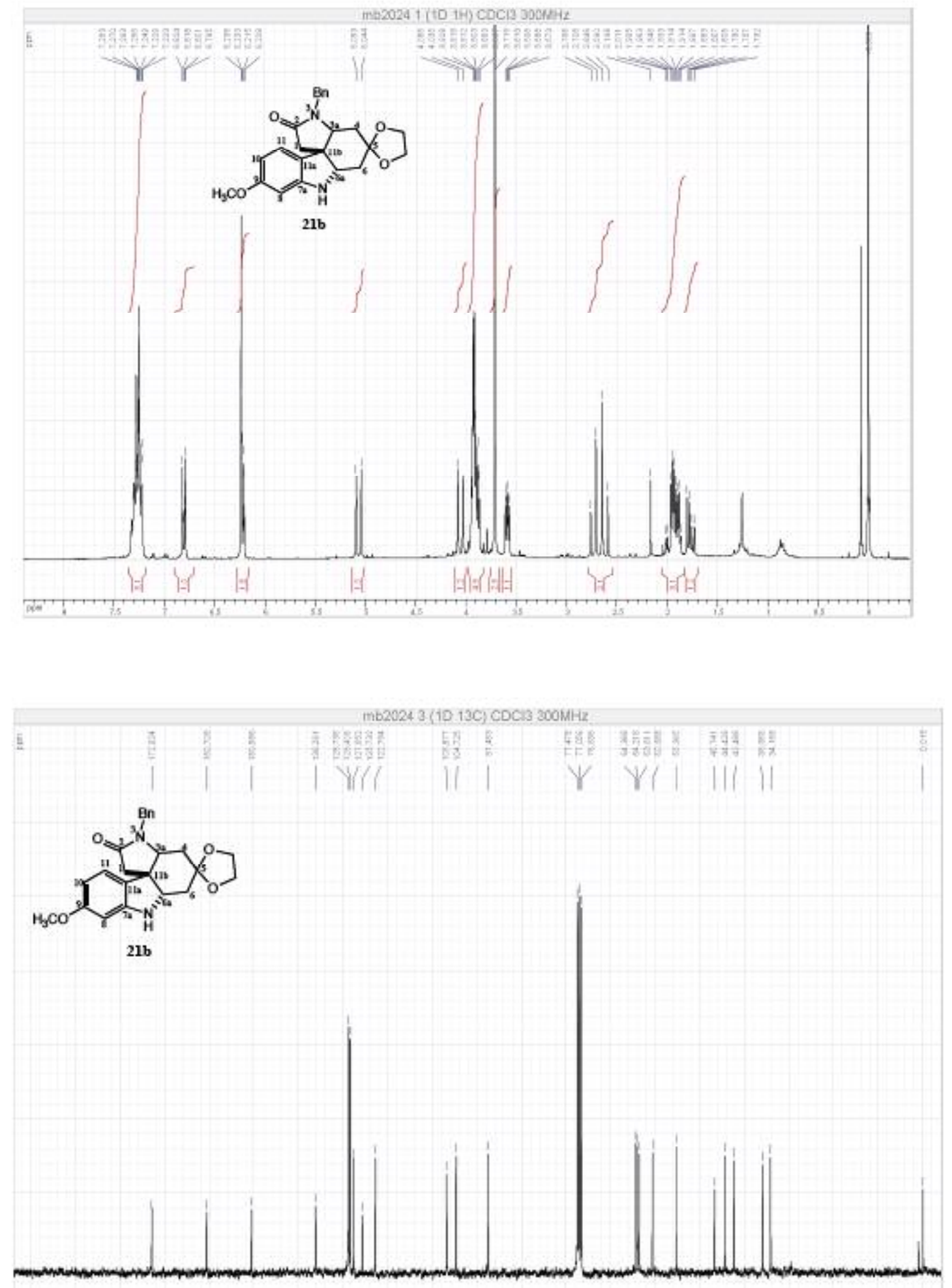

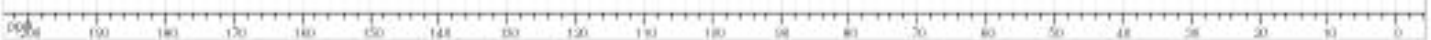



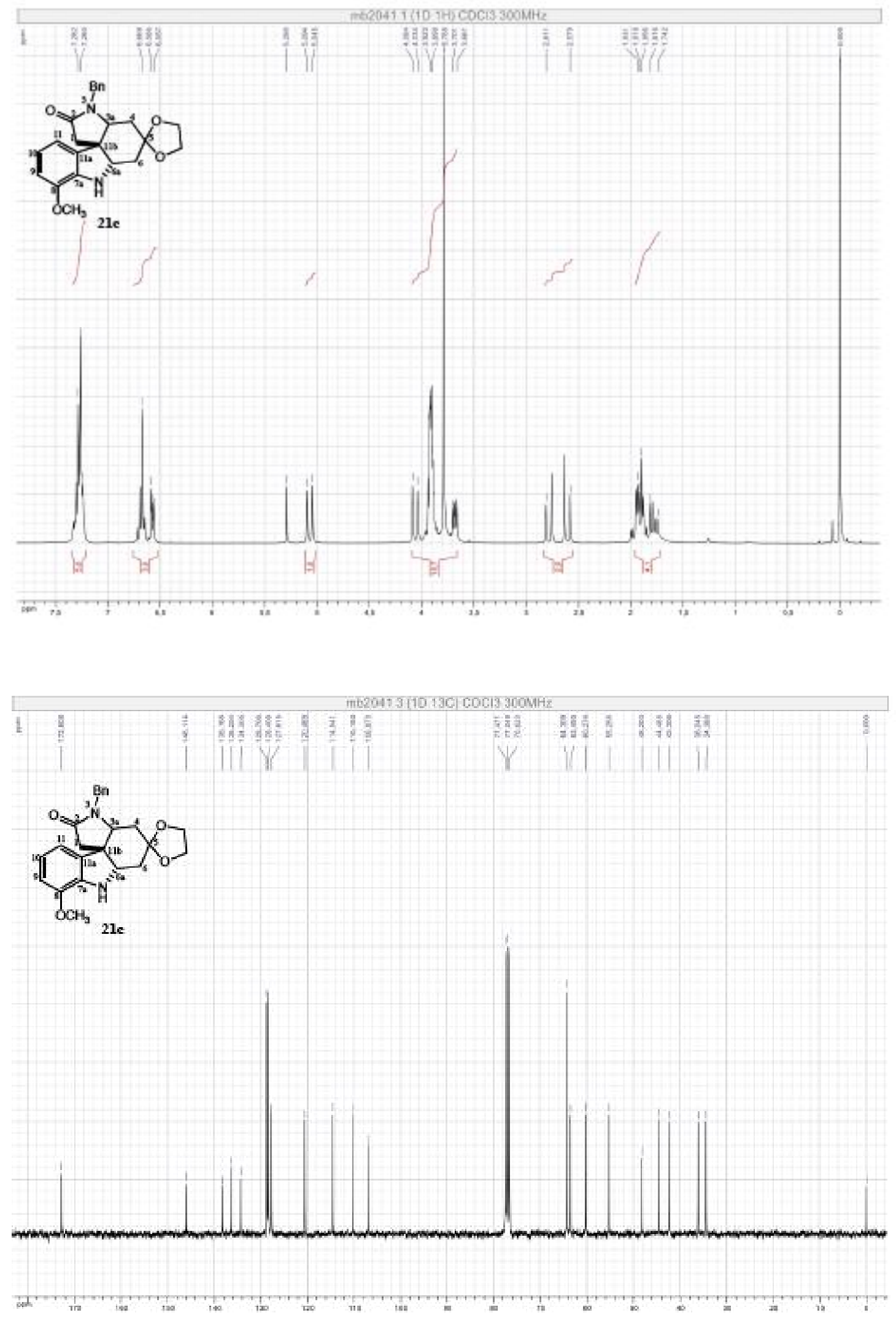


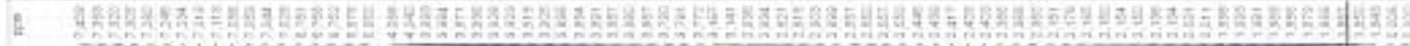
Til if if
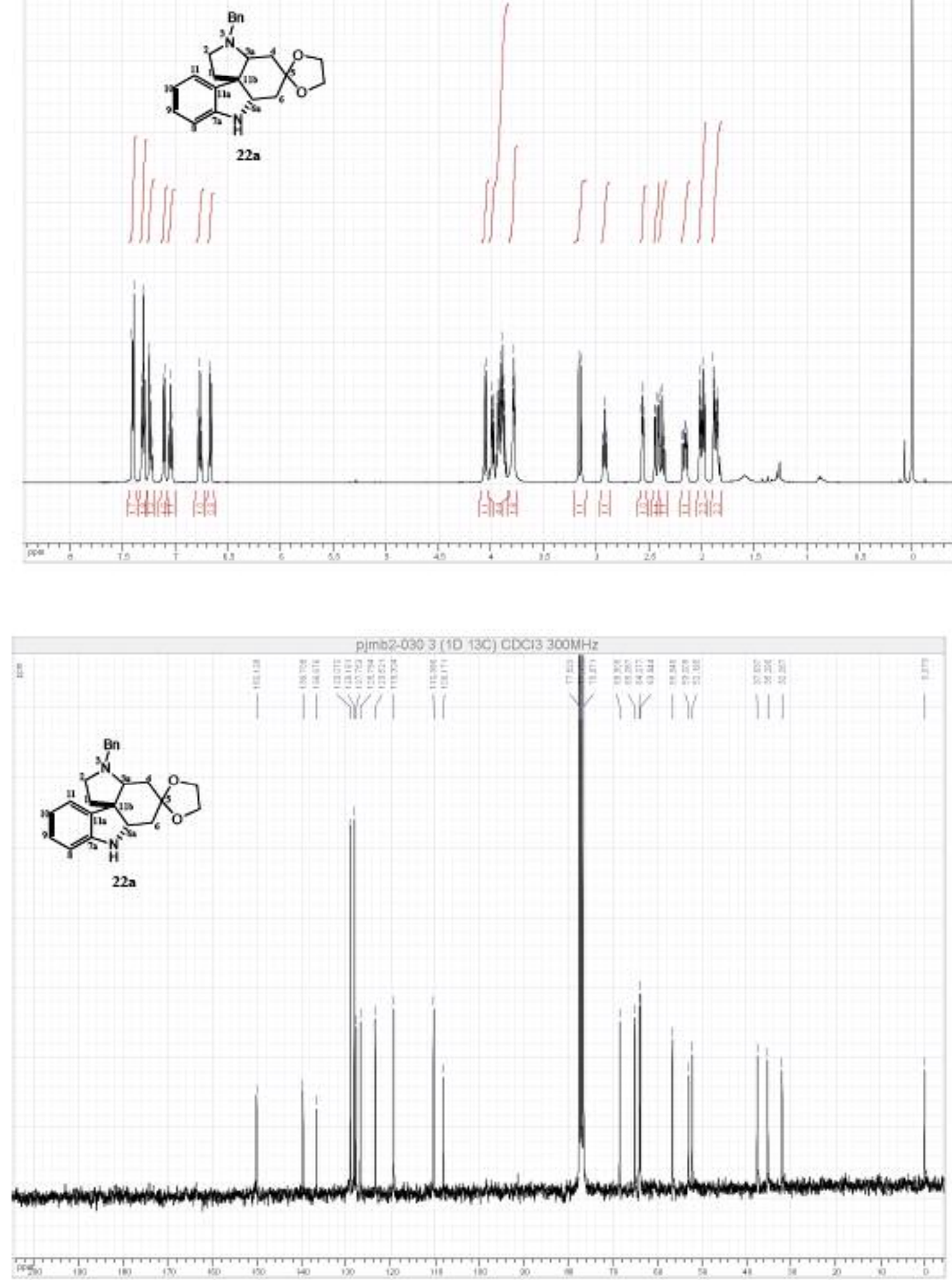


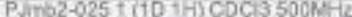

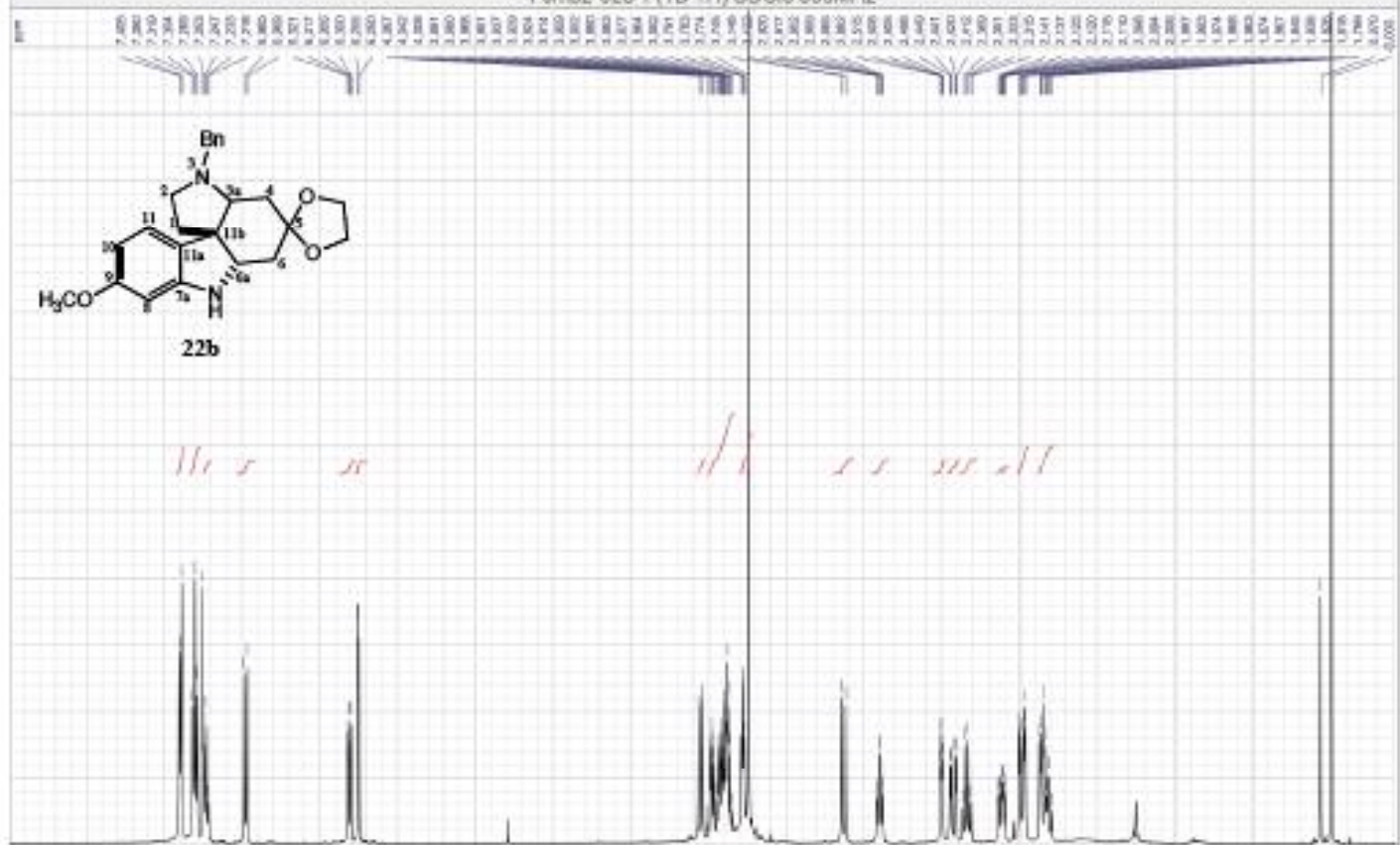

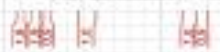

Anatin

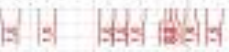
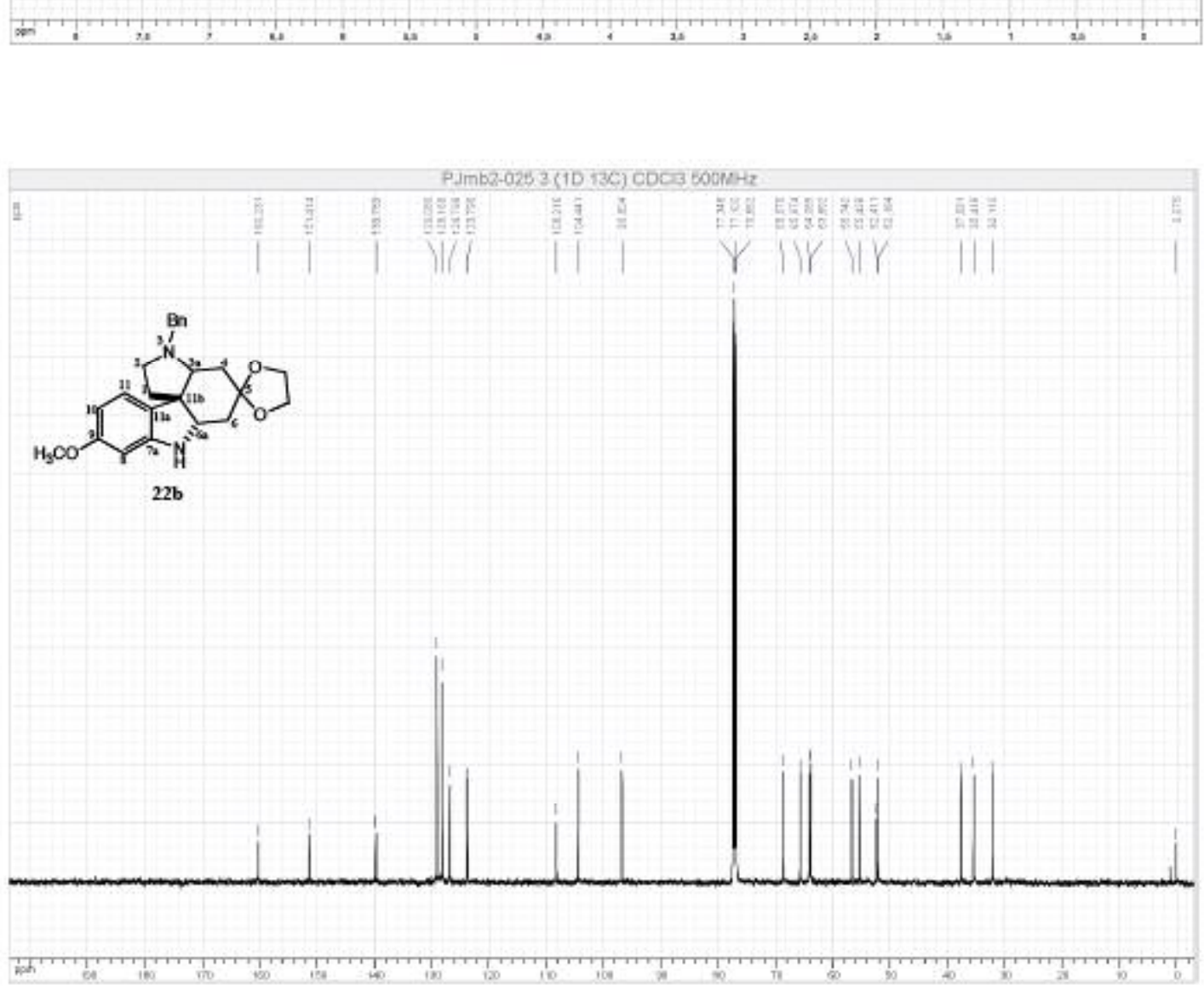

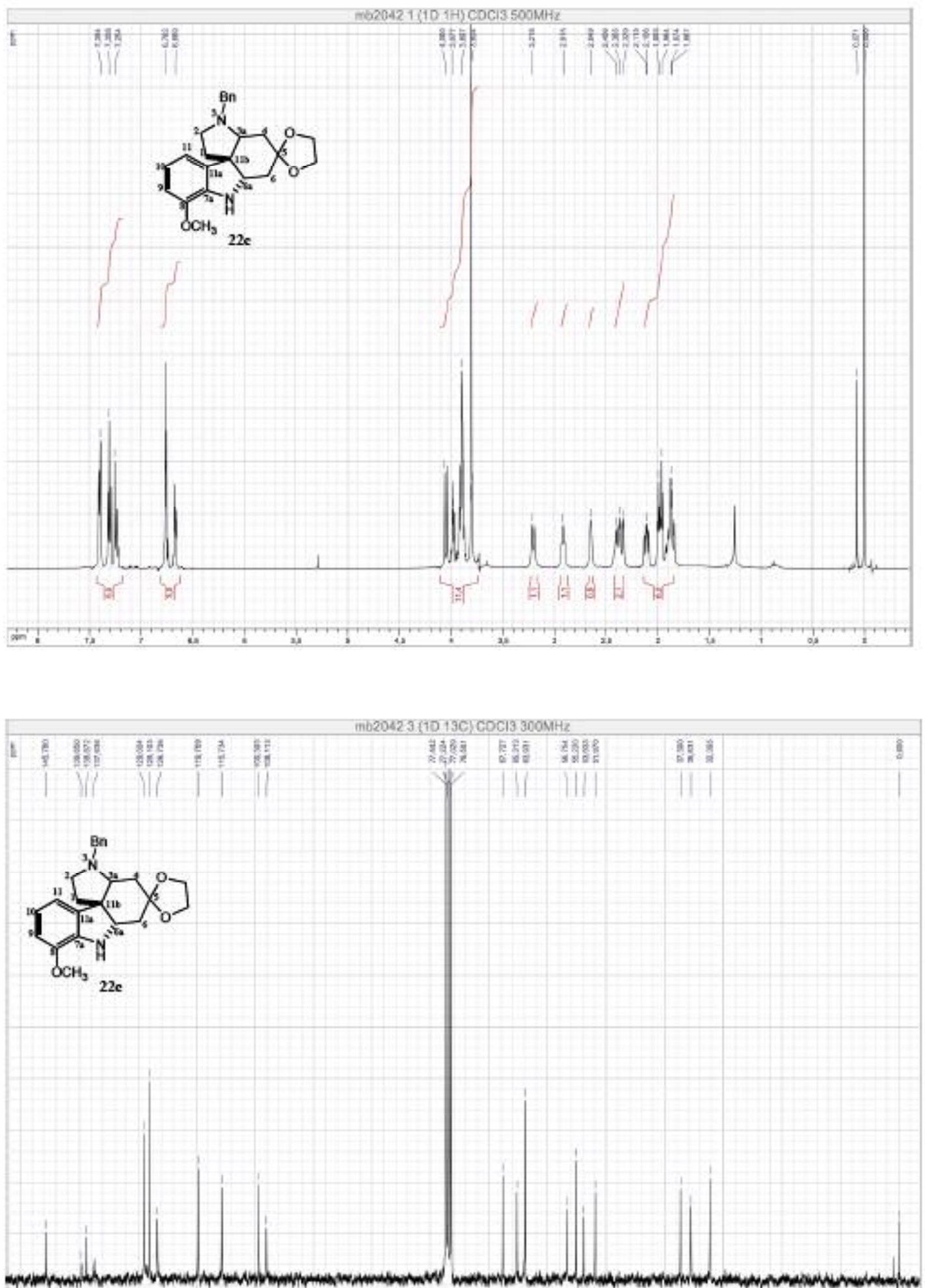

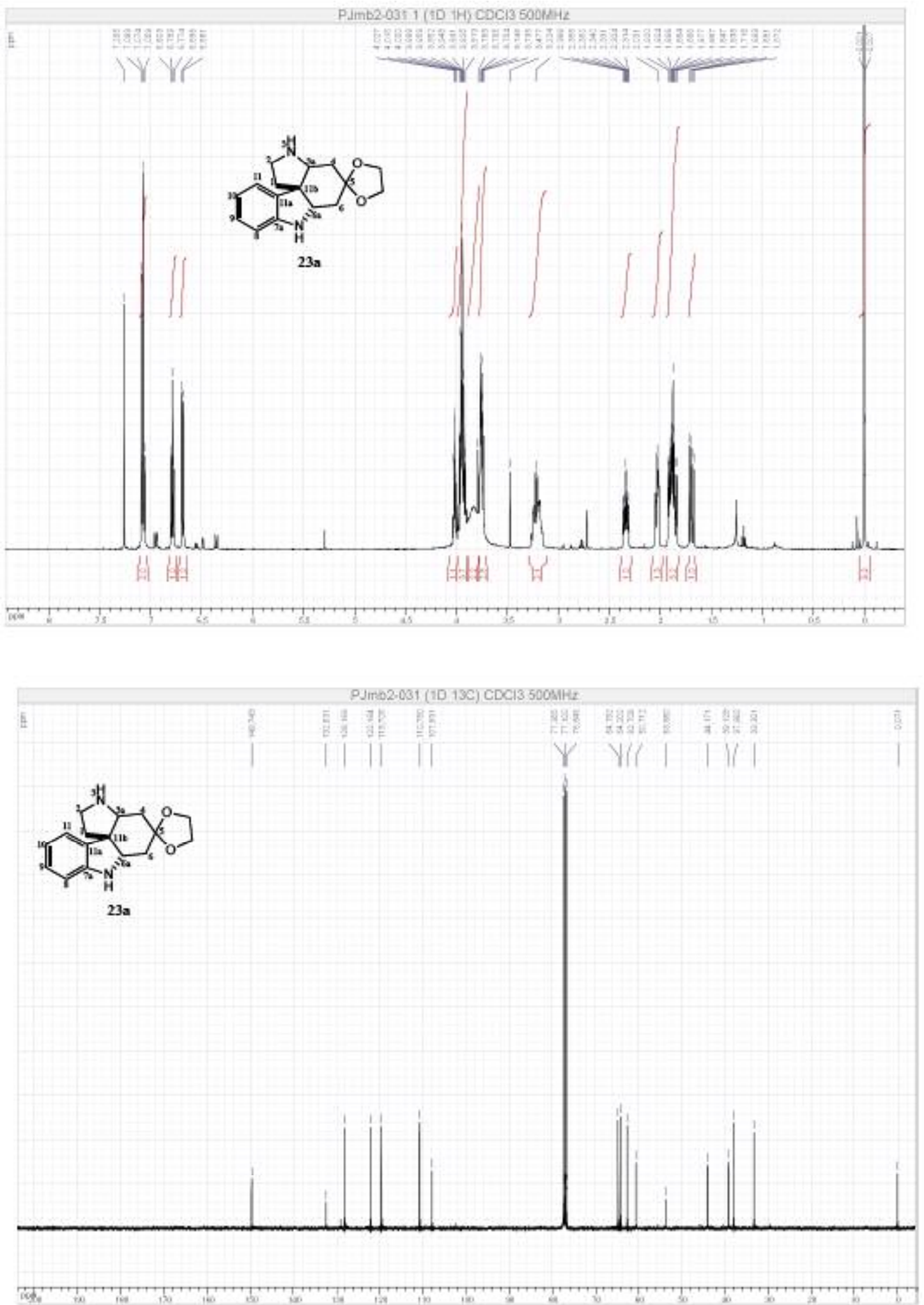

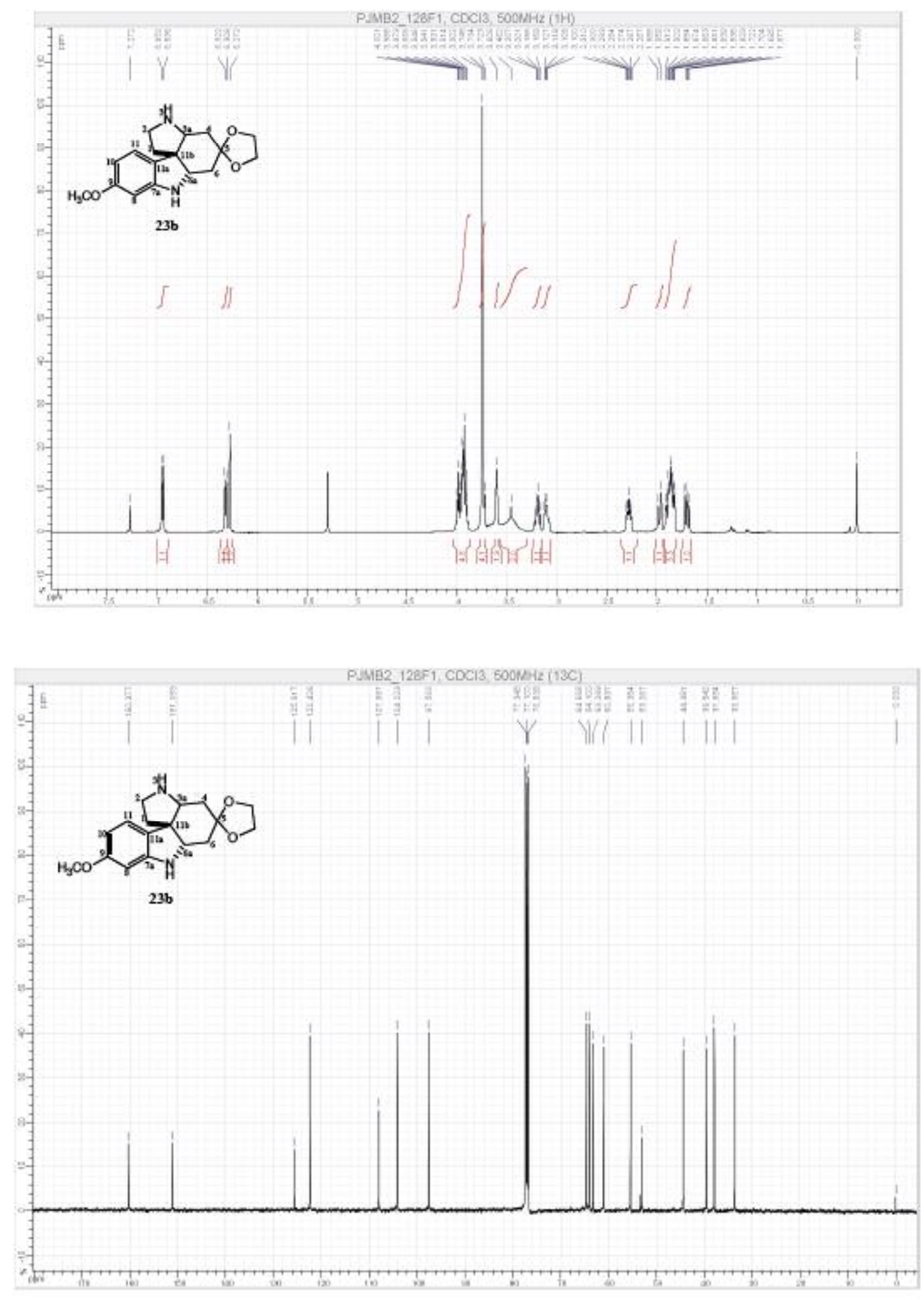

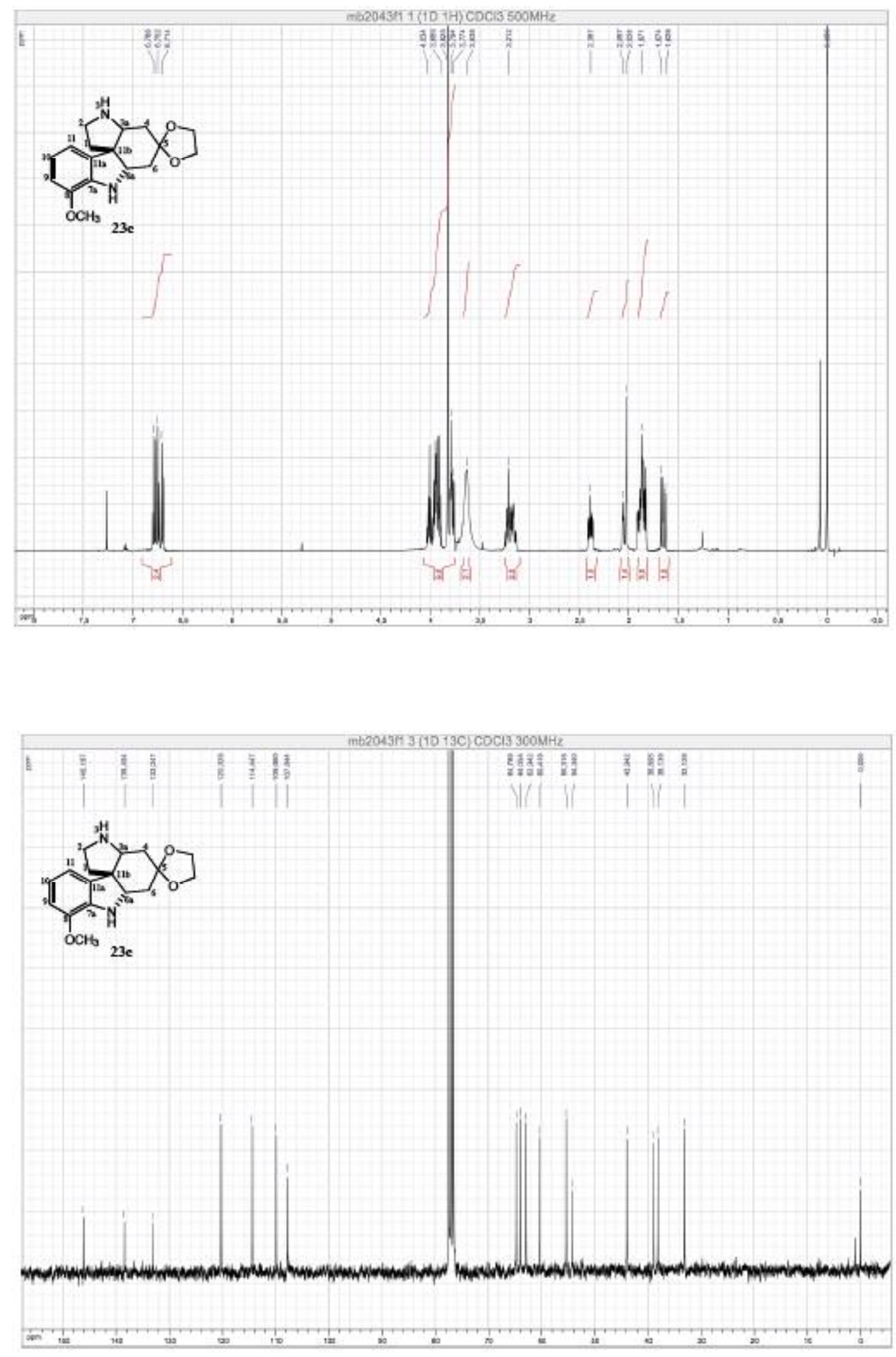

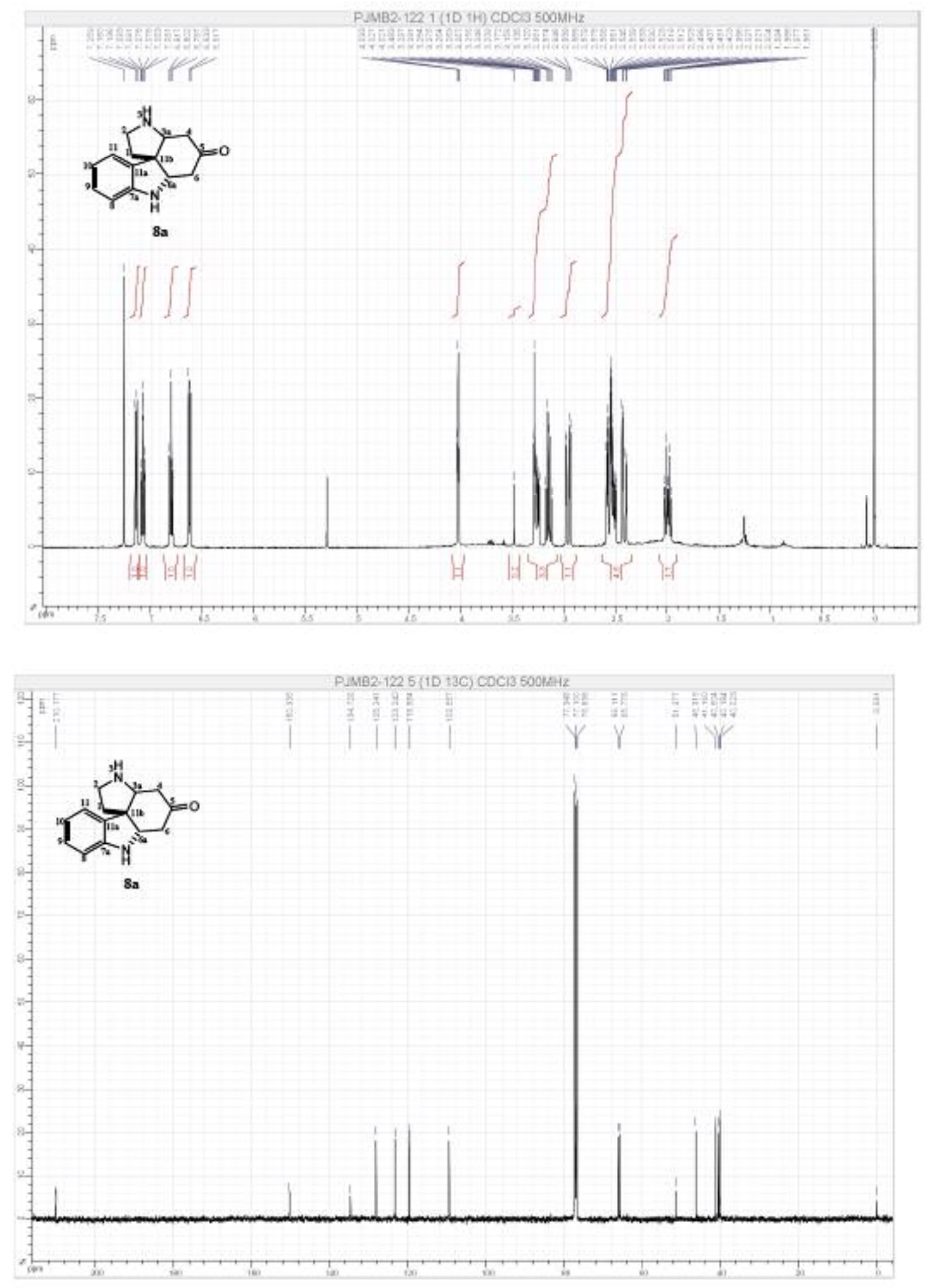

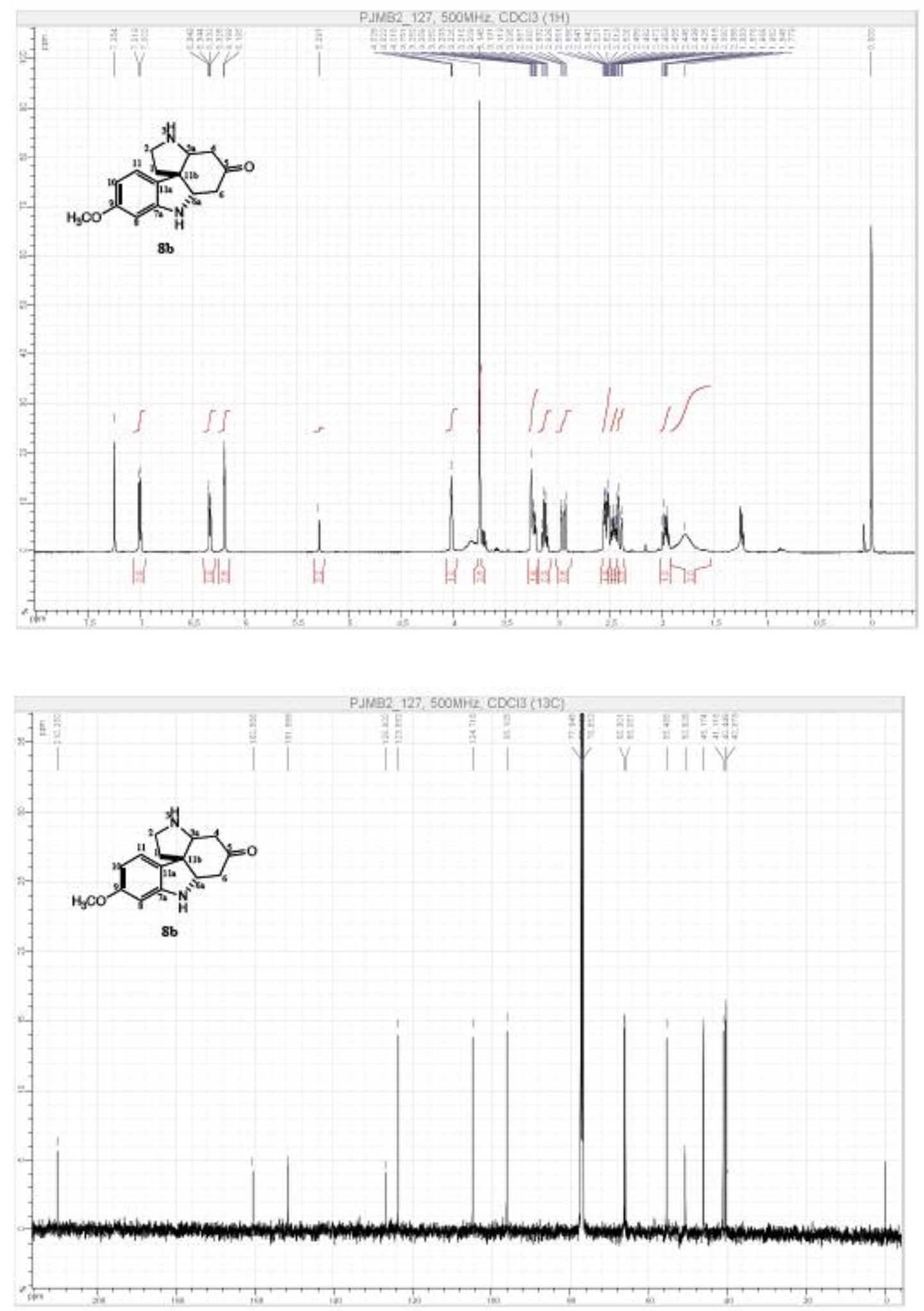

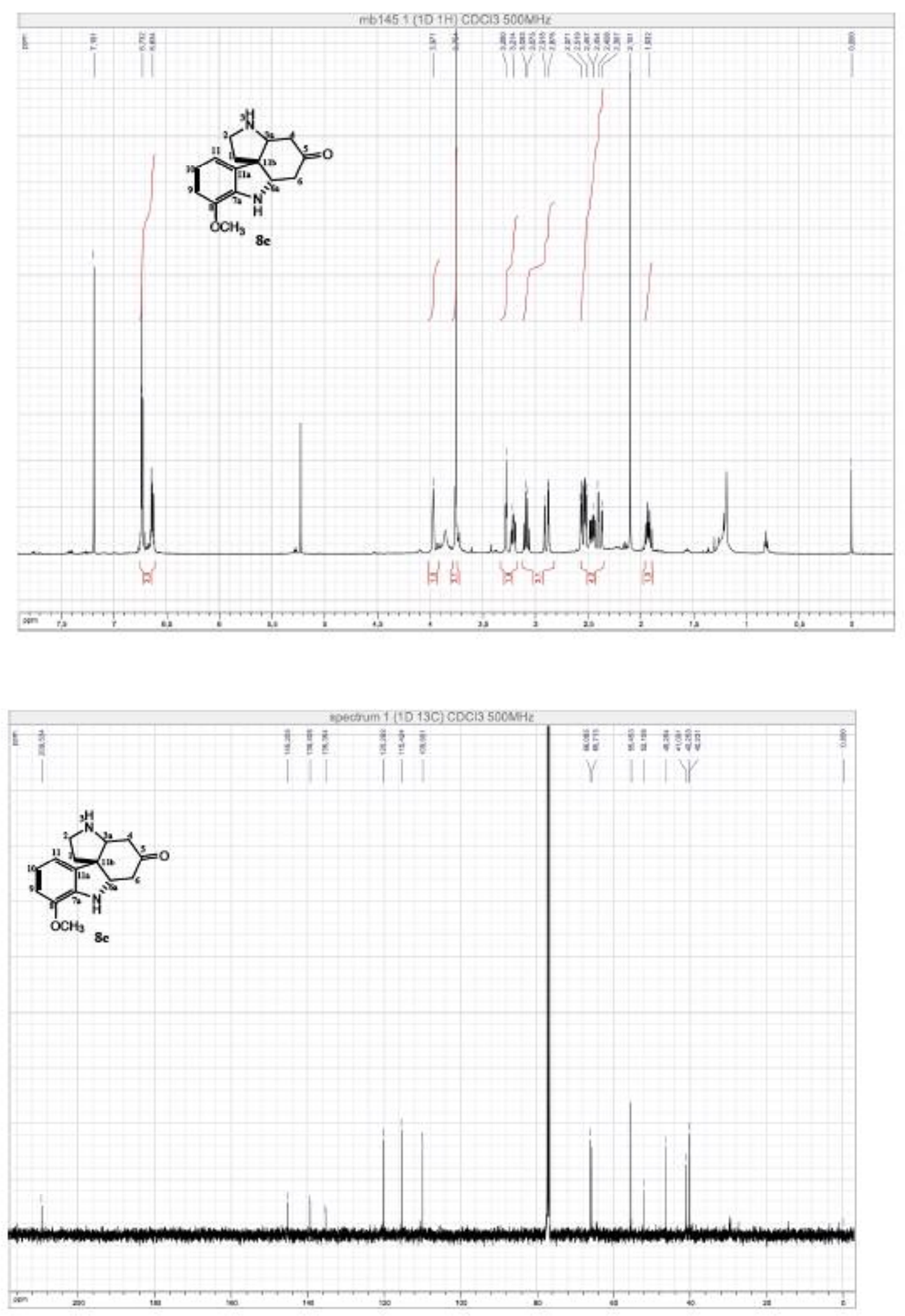